\title{
Optimization of Brain and Head \& Neck Radiotherapy
}

Citation for published version (APA):

Eekers, D. B. P. (2018). Optimization of Brain and Head \& Neck Radiotherapy. [Doctoral Thesis, Maastricht University]. Datawyse / Universitaire Pers Maastricht. https://doi.org/10.26481/dis.20181207de

Document status and date:

Published: 01/01/2018

DOI:

10.26481/dis.20181207de

Document Version:

Publisher's PDF, also known as Version of record

\section{Please check the document version of this publication:}

- A submitted manuscript is the version of the article upon submission and before peer-review. There can be important differences between the submitted version and the official published version of record.

People interested in the research are advised to contact the author for the final version of the publication, or visit the DOI to the publisher's website.

- The final author version and the galley proof are versions of the publication after peer review.

- The final published version features the final layout of the paper including the volume, issue and page numbers.

Link to publication

\footnotetext{
General rights rights.

- You may freely distribute the URL identifying the publication in the public portal. please follow below link for the End User Agreement:

www.umlib.nl/taverne-license

Take down policy

If you believe that this document breaches copyright please contact us at:

repository@maastrichtuniversity.nl

providing details and we will investigate your claim.
}

Copyright and moral rights for the publications made accessible in the public portal are retained by the authors and/or other copyright owners and it is a condition of accessing publications that users recognise and abide by the legal requirements associated with these

- Users may download and print one copy of any publication from the public portal for the purpose of private study or research.

- You may not further distribute the material or use it for any profit-making activity or commercial gain

If the publication is distributed under the terms of Article $25 \mathrm{fa}$ of the Dutch Copyright Act, indicated by the "Taverne" license above, 
Optimization of

Brain and Head \& Neck

Radiotherapy 
(C) DBP Eekers, Vijlen 2018, All rights reserved

Cover: "Emma" by Pascal Eekers

Layout: Tiny Wouters Lenssen

Production: Datawyse Universitaire Pers Maastricht

ISBN: 978-94-6380-036-5

DOI: 10.17915/candat.2018.12.7 


\section{Optimization of \\ Brain and Head \& Neck \\ Radiotherapy}

PROEFSCHRIFT

ter verkrijging van de graad van doctor aan de Universiteit Maastricht, op gezag van de Rector Magnificus, Prof. dr. Rianne M. Letschert, volgens het besluit van het College van Decanen, in het openbaar te verdedigen op vrijdag 7 december 2018 om 10.00 uur

door

Daniëlle Berty Petra Eekers 


\section{Promotores}

Prof. dr. P. Lambin

Prof. dr. D. De Ruysscher

Prof. dr. dr. E.G.C. Troost, OncoRay, Dresden, Duitsland

\section{Copromotor}

Dr. F. Hoebers

\section{Beoordelingscommissie}

Prof. dr. ir. F. Verhaegen (voorzitter)

Prof. dr. K. Herfarth, UniversitätsKlinikum Heidelberg, Duitsland

Prof. dr. M. Klein, Vrije Universiteit Medisch Centrum, Amsterdam

Prof. dr. Y. Temel

Prof. dr. J.E. Wildberger 
Voor Imke, Karsten \& Erik, jullie geven de glans 



\section{Contents}

$\begin{array}{lll}\text { Chapter } 1 & \text { Introduction } & 9\end{array}$

Chapter 2 Benefit of particle therapy in re-irradiation of head and neck 25 patients. Results of a multicentric in silico ROCOCO trial

Chapter 3 Intensity-modulated proton therapy decreases dose to organs at risk in low-grade glioma patients: results of a multicentric in silico ROCOCO trial

Chapter 4 The posterior cerebellum, a new organ at risk?

Chapter 5 The EPTN consensus-based atlas for CT- and MR-based contouring in neuro-oncology

Chapter 6 Radiation dose constraints for organs at risk in neuro-oncology; the European Particle Therapy Network consensus

Chapter 7 Evidence on the efficacy of primary radiosurgery or stereotactic radiotherapy for drug-resistant non-neoplastic focal epilepsy in adults: A systematic review

Chapter 8 General discussion

Chapter 9 Valorisation

Summary / samenvatting

Dankwoord 



\section{Chapter 1}

General introduction 



\section{Introduction}

Radiotherapy is one of the three pillars of anti-cancer therapy, besides surgery and systemic therapy. It uses ionising radiation to damage tumour cells by causing a plethora of effects, the most prominent being double strand breaks in the DNA of the cell. Most often, external beam radiotherapy, i.e., ionising irradiation applied from outside the body generated by a linear accelerator, is used. Thus, the ionising radiation needs to travel through the body to reach the tumour and will therefore damage not only tumour cells, but may also harm normal tissue cells, on its path. As opposed to tumour cells that are not properly equipped to recognise and repair the damages caused by radiation, healthy normal cells detect the damage and are able to repair it. A prerequisite for this is that the dose per fraction of radiation administered is to be kept reasonably low, e.g., 2 Gray (Gy), and consequently a full treatment is delivered over the course of several weeks. Nevertheless, not all healthy cells will survive, resulting in acute and long-term side effects of the so-called organs at risk (OAR). Most acute side effects recuperate in the period of weeks until three months after radiotherapy, however, the long-term side effects develop after months to years and are often irreversible. So, the aim of radiotherapy is to give a lethal dose to the tumour and to spare the surrounding OARs as much as possible in order to reduce the likelihood of acute and especially long-term side-effects.

\section{Developments in radiotherapy}

Since tumours have irregular shapes and are surrounded by healthy tissue, radiation technology needs to enable the radiation oncologists and physicists to view the tumour in three dimensions, to sculpt the beams to its shape, and to employ steep dose gradients sparing the OARs. By using multiple beams, the tumour is faced by the sum of these beams delivering a high dose to tumour, while the dose to the OARs in the tract of each beam is kept reasonably low. This technique is called 3D conformal radiotherapy (3D-CRT).

In the passing decade, radiotherapy has evolved from 3D-CRT to intensity-modulated radiation therapy (IMRT). In IMRT, one radiation beam is divided into multiple small beams, so-called beamlets. The intensity of these beamlets can be modulated over time in order to give an optimal dose distribution to the tumour and to spare the OARs. Over the last years, new rotational IMRT technologies have become available, such as volumetric modulated arc therapy (VMAT) and helical Tomotherapy (TOMO) $)^{1-3}$. VMAT and TOMO are a further development of IMRT, such that the linear accelerator rotates around the patient while the intensity of the radiation, the velocity of machine rotation 
and the shape of the radiation field can vary during treatment. In most cases, while using VMAT, the machine is able to deliver the dose faster than when using IMRT, with a dose distribution that is often even more conformal to the tumour. VMAT can be delivered with standard gantry-based linear accelerators. Alternatively, it can be delivered using a linear accelerator combined with a computed tomography scanner (CT), rotating in a helix around the patient, the so-called TOMO system. In the previously described treatment modalities such as 3D-CRT, IMRT, VMAT and TOMO, high-energy photons are used to irradiate the tumour. Photons are packages of energy and as such have no mass or charge, delivering their dose to the tissue along the beam path by causing secondary electrons in the patient's body.

Still, it is inevitable that the OARs frequently receive a clinically significant dose with these techniques, especially when rotational photon-based ${ }^{2,4}$. In an attempt to reduce the dose to the OARs further, particle therapy (PT) is increasingly being introduced in clinical practice. Charged particles, such as carbon-ions and protons have a mass and are charged, which causes a different dose distribution in the patient. The particles have a low entry dose followed by an energy-dependent maximum dose delivery at the so-called Bragg peak with low to zero dose after the dose fall-off at the distal edge of the Bragg peak (see Figure 1.1). Particle therapy potentially leads to a superior sparing of the surrounding OARs compared to photons, a hypothesis that needs to be tested and confirmed in (in silico) clinical studies (this thesis). These studies can be designed as register studies, in a randomised fashion, or by model-based treatment selection.

Proton beam irradiation is delivered using a so-called cyclotron. In this cyclotron protons are accelerated outwards from the centre along a spiral path, in which they are held by a static magnetic field. In recent years, manufacturers of cyclotrons were able to reduce the size of these machines considerably and therefore increase the affordability of this relatively novel technique. Consequently, proton beam therapy is increasingly available throughout the world and prompts interest among physicians as well as health insurance companies regarding its therapeutic efficacy and possible toxicity reduction. There are currently two proton-beam delivery methods:

Passive scattered proton therapy (PSPT), and active proton therapy or pencil beam scanning (PBS). In PSPT, a modulator wheel placed in the beam path degrades the proton energy and spreads out the Bragg-peak (SOBP). Furthermore, individual patientand beam-specific modifying devices, i.e. compensator and collimator, have to be designed to conform the dose to the target volume. In PBS, magnets are used to steer a small exiting pencil beam in horizontal and vertical direction, such that the target volume is 'painted' with dose. By adjusting the energy of the beam, the depth of the 
Bragg-peak can be adjusted, creating a 3D dose volume with successive treatment layers to conform to the tumour. For PBS, no patient-specific-beam modulators are needed, however, its application to moving targets, such as lung tumours, is currently limited.

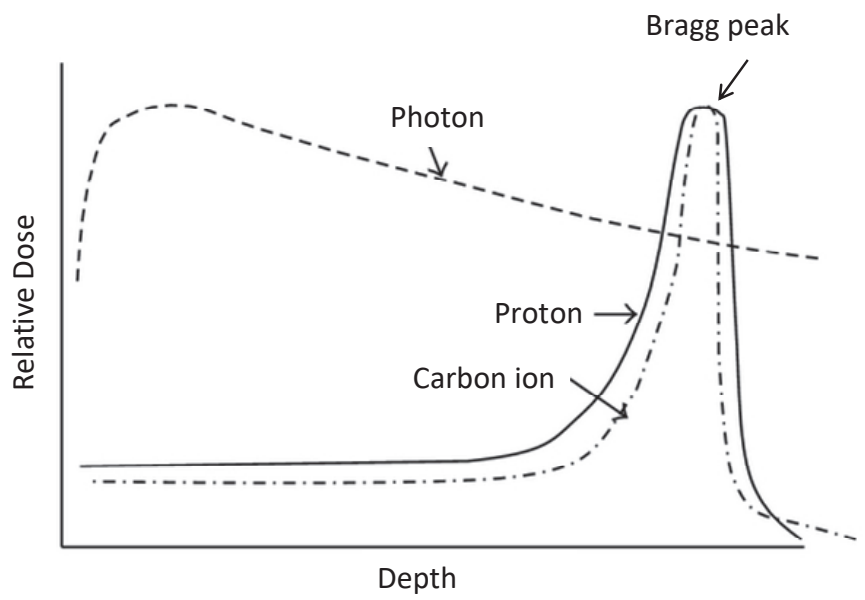

Figure 1.1 Relationship of depth and relative dose in photon (dashed line), proton (solid line) and carbon ion (dashed/dotted line) beams. Proton and carbon ion beams deposit the maximum dose in the so-called Bragg-peak.

Changing the beam intensity during treatment, thus resembling IMRT, is also possible in particle therapy: using protons this is called intensity-modulated proton therapy (IMPT) and using carbon ions, intensity-modulated ion therapy (IMIT). Due to the large impact of acute and late radiation-induced toxicities in patients with tumours originating from the brain or head-and-neck, these sites are of particular interest for the use of IMPT and IMIT.

\section{Head and neck}

Squamous cell carcinoma of the head and neck (HNSCC) is the sixth most prevalent cancer in the world and remains difficult to cure ${ }^{1,5}$. The treatment of the primary tumour and lymph nodes includes either primary surgical resection with or without postoperative RT or primary RT. RT can be combined with systemic treatment, depending on various patient and tumour characteristics.

Unfortunately, all treatment modalities applied with curative intent cause side effects. Surgery might cause temporary or permanent impaired speech, swallowing or 
breathing impairment, endocrine dysfunction, or nerve damage (often facial nerve or accessory nerve). Radiotherapy as a part of organ preservation strategy in HNSCC may cause acute skin (redness, epidermolysis) and mucosal (mucositis) damage, xerostomia, taste loss, hypothyroidism, dysphagia, fibrosis, and fatigue or pain. Some of these side effects might recover in time and others remain after treatment.

The risk of local or regional recurrence after having undergone radio(chemo)therapy with or without surgery is still up to $30 \%$ despite many new treatment options ${ }^{6-8}$. In case of irresectable recurrent disease in the primary tumour or lymph nodes, one of the potentially curative treatment options is re-irradiation (re-RT), sometimes combined with systemic therapy. However, also after re-RT, the rates of severe grade 3 or 4 toxicity are high, with an incidence of up to $45 \%$ at 5 years, and only 1 in 3 patients surviving re-RT without recurrence and severe complications ${ }^{9}$. Reduction of this risk of radiation induced complications and improvement of the oncological outcome is needed ${ }^{9-15}$.

\section{Primary brain tumours / gliomas}

Gliomas are the most common tumours of the central nervous system (CNS), with an annual incidence of 5.4 per 100.000 in Europe ${ }^{16}$. They can be classified according to the World Health Organization (WHO) grading system from grade I to IV ${ }^{17}$. Grade I is pathologically benign and grade IV the most malignant glioma, also called a glioblastoma. Low-grade glioma (LGG), classifying as WHO grade II, are diffusely infiltrative tumours and typically occur in younger adults ( 2 nd-5th decade). Although most LGG show a protracted disease course, differences in survival rates have been identified. Molecular parameters, such as 1p19q co-deletions, isocitrate dehydrogenase (IDH) mutation, telomerase reverse transcriptase (TERT) promotor mutations, and epidermal growth factor receptor (EGFR) amplifications and mutations have been integrated in the WHO classification of CNS tumours revised in 2016, which helps to more accurately predict prognosis ${ }^{18-20}$. This integrated diagnosis of gliomas is now leading the neuro-oncology field towards multimodal treatment schedules capable of increasing overall survival of diffuse gliomas with an aggressive clinical behaviour ${ }^{21,22}$. Multimodal treatment schedules include neurosurgical resection if feasible and adjuvant treatment with radiation therapy as well as chemotherapy ${ }^{22}$. The surgical resection of a large part of the tumour (gross total resection) is not without risk and is in some cases performed while the patient is awake in order to preserve as much function as possible. There is a chance of bleeding, neurological deficits such as paralysis, cranial nerve palsy or vision loss depending on the location and infection. 
Radiotherapy may cause headache, nausea, vomiting, fatigue and hair loss during treatment. The long-term the side effects of radiotherapy can include necrosis of healthy tissue (radio-necrosis) and cognitive decline. With increasing survival of LGG patients, therapy-induced toxicity, which subsequently decreases the quality of life of these patients, should be minimised ${ }^{23}$.

Neurocognitive decline, which is marked by reduction of verbal memory, spatial processing, attention and novel problem-solving ability, is probably the most relevant (long-term) complication following (radio)therapy of the brain ${ }^{24}$. An important part of the limbic system, which is involved in emotions and the forming of memories, are the hippocampi, which play an important role in cognition ${ }^{25}$. Damage to the hippocampi, as is the case in Morbus Alzheimer, results in loss of short-term memory and disorientation. When both hippocampi are affected, even a total loss in the ability to create new memories may occur, called an anterograde amnesia. There is some evidence that besides the hippocampi, the cerebellum plays a role in neurocognitive function ${ }^{26}$. Traditionally, the cerebellum is known for its role in regulation and coordination in movement, posture and balance ${ }^{26}$. However, several clinical, anatomical and neuro-imaging studies have shown that the cerebellum, especially the cerebellum posterior, may also play a role in neurocognition ${ }^{27-29}$.

\section{Epilepsy}

The most frequent symptom of patients with LGG is epilepsy in up to $65-90 \%$ of cases $^{30,31}$. Having seizures causes a great emotional burden to a patient and decreases the quality of life significantly ${ }^{32}$. Epilepsy in general is one of the most common severe neurological disorders. The WHO has estimated that more than 50 million patients suffer from epilepsy worldwide ${ }^{33-35}$. Epileptic seizures are episodes of uncontrolled vigorous shaking as a result of excessive and abnormal neuronal activity in the cortex of the brain ${ }^{36}$. Epilepsy can be treated successfully with anti-epileptic drugs (AED) or in selected cases of AED-resistant patients, with surgery removing the epileptogenic focus. In patients with primary brain tumours, treating the tumour, e.g., with radiotherapy, has been shown to have a positive effect on the epilepsy ${ }^{37}$.

Reduction in epilepsy frequency after treatment can be classified using the Engel classification from I-IV. Class I meaning that a patient is completely seizure free for at least 2 years, class II only rare seizure ( $\leq 2$ seizures per year) remain, class III is considered worthwhile improvement and class IV meaning no significant improvement or even increase in seizure frequency.

Besides the fact that a certain epilepsy reduction can be achieved with radiotherapy in the presence of a tumour, patients with epileptic lesions without having a tumour can 
also be treated with radiotherapy. These so-called non-neoplastic focal epileptic lesions (NNFE) are associated with focal seizures, which have a localised, well-circumscribed network of discharges and can be treated successfully with radiotherapy, besides receiving treatment with anti-epileptic drugs and resective surgery ${ }^{38,39}$. The success rate is around $50 \%$ (this thesis) whereas the postoperative seizure free outcome varies between $60-90 \%$ depending, among others, on the pathological substrate ${ }^{40}$.

Radiotherapy as a non-invasive approach may be a good alternative to surgery when the epileptogenic region is located near the eloquent cortex or deeply sited brain areas and this region is too hazardous to resect ${ }^{40,41}$. Several publications have underlined the potential value of this alternative therapy in patients with drug-resistant epilepsy, although currently only few radiotherapy institutes in the world treat patients for epilepsy ${ }^{40,42-44}$. Having a feasible and non-invasive alternative for patients for whom surgery is no option is of great importance.

\section{Challenges in radiotherapy}

With all new developments in health care treating malignant or benign diseases, a tailor-made treatment selection for every individual patient is desperately needed. Therefore, it is crucial to find the evidence to support this decision-making, in particular between two treatment modalities with relevant differences in costs or toxicity profiles. Carefully performed randomised trials of two seemingly equal treatments could provide us with the evidence we need.

When considering proton therapy, however, randomised trials are increasingly difficult to perform mostly due to costs and availability. To deal with this dilemma, a new approach was introduced in the Netherlands, the so-called model-based approach ${ }^{45}$. This approach aims at selecting those patients most likely to benefit from a specific treatment, in this case proton beam therapy, using pre-treatment plan comparison and translating a dose distribution into anticipated clinical benefit using validated normal tissue complication probability (NTCP) models. The maturation and validation of NTCP models is strongly dependent on uniform delineation of the relevant OARs (this thesis). An NTCP model uses data on dose to OARs of previously treated patients and relates these to their reported toxicity. With such a model it is possible to estimate the individual patient's potential benefit of applying one treatment instead of another in terms of expected reduction of radiation-induced side effects, knowing the dose to a specific OAR ${ }^{16,46-48}$. Noteworthy, these NTCP models need to be validated and further developed using the outcome of clinical trials. 
Further reduction of these therapy-induced side-effects should be one of the main goals of future research in radiotherapy. With all kinds of new technological developments, such as cheaper cyclotrons or magnetic resonance-integrated linear accelerators (MR-linac), we need a way to predict and compare the risk on side effects in order to select the right treatment modality, for the right patient. This is a necessity for photon as well as for particle therapy. There are many radiotherapy centres; in 2012 Europe had 1286 registered radiotherapy centres in the Directory of Radiotherapy Centres database ${ }^{49}$. Moreover, according to the particle therapy co-operative group (PTCOG) database, there are now 25 particle therapy facilities in Europe of which 11 are under construction. Until now, the neurological oncology community has not succeeded in collecting sufficient valid data to build a validated NTCP model for tumours of the central nervous system as opposed to the dysphagia and xerostomia NTCP models for the primary treatment of head and neck tumours (see Figure 1.2) ${ }^{50-53}$.
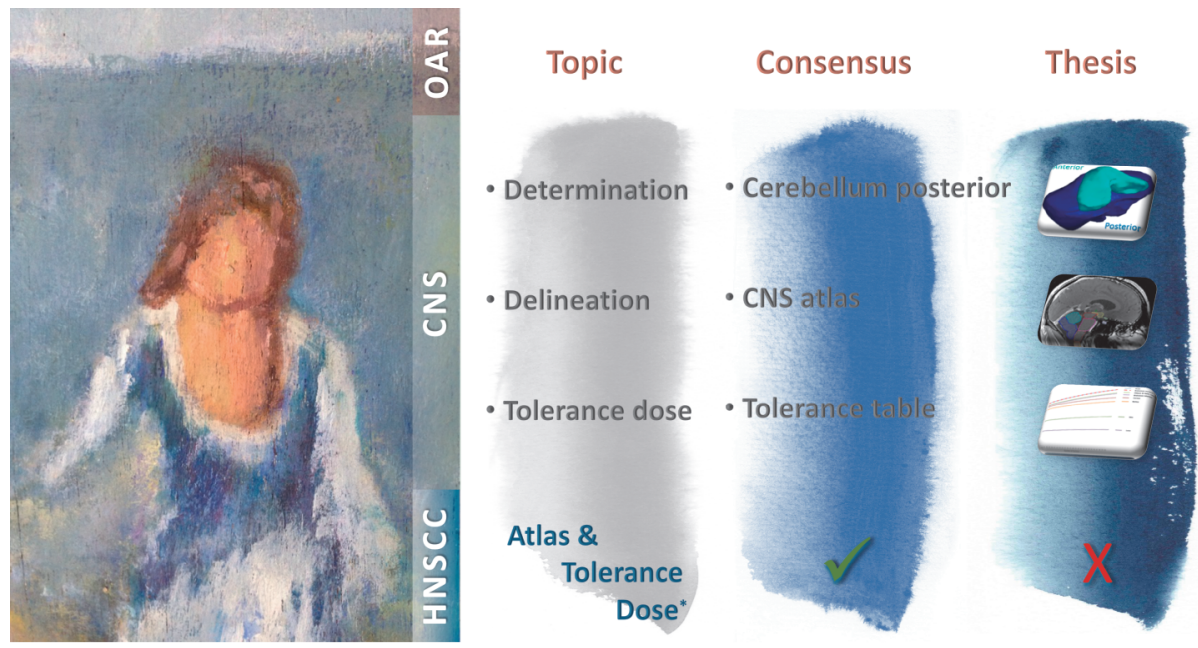

Figure 1.2 The central nervous system (CNS) and head and neck squamous cell carcinoma (HNSCC) status on consensus for organs at risk (OAR) determination, delineation and tolerance dose in relation to the outcome of this thesis. $\mathrm{V}=$ present in literature $\left({ }^{*}\right.$ Brouwer et $\left.a{ }^{5} .{ }^{54}\right) . \mathrm{X}=$ not a subject of this thesis. Painting in private possession.

Since brain tumours are rare and some side effects take years to develop, international cooperation and agreement is needed on 3 topics: first, on the identification of the relevant OARs; second, on the delineation of these OARs represented in an atlas; and third, on the tolerance dose of the identified OARs. Consensus on outcome reporting 
measures, i.e., uniform follow-up timing, patient questionnaires and content of the follow-up, is mandatory as well ${ }^{55-58}$. In an attempt to reach these ideal conditions for comparison, a list of the OAR's distinct radiation-induced toxicities and the recommended dose constraints for conventionally fractionated radiotherapy is needed, for it has been a while since the recommendations by Emami et al. ${ }^{59}$ and the QUANTEC series $^{60-63}$ were published. A solid data collection is also mandatory of all treated patients, including registration of the treatment modality (photon or particle), dose distribution and uniform registration of the side effects.

Recently, a taskforce from the European Society for Radiotherapy \& Oncology (ESTRO) was established called the European Particle Therapy Network (EPTN). Within the EPTN, forces are joined to share knowledge and stimulate cooperation between the particle centres, for example on clinical trials in Work Package 1 (WP1).

\section{Objective of this thesis}

The overall objective of this doctoral thesis was to assess the value of proton beam therapy, in particular for re-irradiation in HNSCC and primary irradiation of LGG, see Figure 1.3. This included a comparative study on reducing the radiation dose to the OARs, and consensus on contouring of OARs and their tolerated dose constraints among European particle centres.

The first objective was addressed within the Radiation Oncology COllaborative Comparison (ROCOCO) initiative. ROCOCO is a consortium of various international centres conducting in silico photons and particles treatment plan comparisons ${ }^{64-66}$. To assess the potential gains due to the dosimetric characteristics of particle therapy for individual patients, two in silico treatment planning studies were conducted within this ROCOCO group (this thesis), one on a cohort of 25 HNSCC patients (clinicaltrial.gov ID: NCT 02242916), the other on 25 LGG patients (clinicaltrial.gov ID: NCT NCT02607397) ${ }^{47}$. The second objective was addressed within the EPTN. A group of expert radiation oncologists in the field of neuro-oncology discussed and reached agreement on which relevant OARs needed to be included in the delineation atlas, and on the tolerance dose of these OARs, in order to be able to uniformly gather data on dose-toxicity profiles in time (this thesis) ${ }^{48,57,58}$. Due to this uniformity, these data can be used in future joined efforts to generate and validate new CNS NTCP models for protons as well as photons.

The third objective was determining the role of radiotherapy in the treatment of drugresistant non-neoplastic focal epilepsy in adults, being a possible indication for particle therapy in the future ${ }^{67}$. 


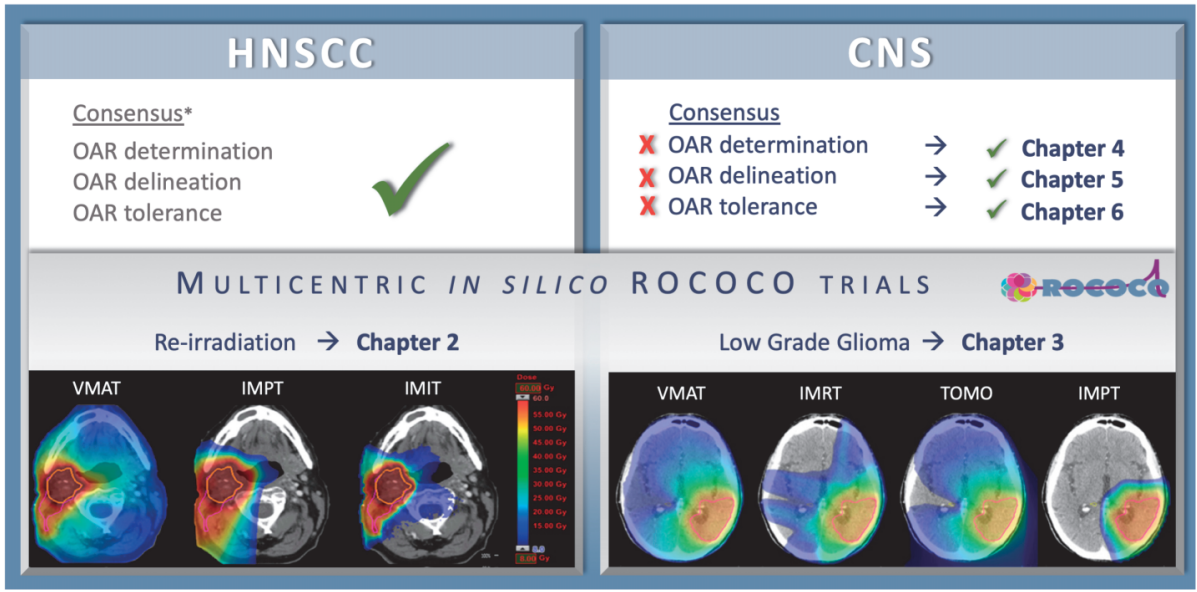

Figure 1.3 The outline of this thesis and the corresponding chapters. OAR = organ at risk, HNSCC = head and neck squamous cell carcinoma, CNS $=$ central nervous system, $*=$ Brouwer et al. ${ }^{54}, \mathrm{ROCOCO}$ = Radiation Oncology COllaborative COmparison group. VMAT = volumetric modulated arc therapy, IMPT = intensity-modulated proton therapy, IMIT = intensity-modulated ion therapy, IMRT = intensity-modulated radiation therapy, TOMO = helical Tomotherapy.

\section{Summary of the chapters}

Chapter 2 reports on the results of the ROCOCO in silico trial comparing IMPT and IMIT with VMAT for re-irradiation of 25 head-and-neck cancer patients. The results showed a reduction in dose to all OARs using particle, in particular favouring carbon-ions ${ }^{47,65}$.

Chapter 3 reports on the results of the ROCOCO in silico trial comparing IMPT, TOMO and IMRT with VMAT for irradiation of 25 low grade glioma patients. The study demonstrated that proton therapy was the most effective in reducing irradiated volumes at low and intermediate dose levels of contralateral OARs. Photons performed better in the high dose area resulting in a better target conformity ${ }^{68}$.

Chapter 4 presents the posterior cerebellum as a possible, new organ at risk relevant for cognitive decline. This literature review summarises the available evidence on its role in cognition, which is in particular relevant for irradiation of head-and-neck cancer patients ${ }^{69}$.

Chapter 5 reports on the EPTN consensus-based atlas for CT- and MR-based contouring in neuro-oncology. Relevant OARs in neuro-oncology and the corresponding delineation instructions are illustrated in a novel online atlas, harmonising current clinical practice $^{54,56}$. 
Chapter 6 reports on the EPTN consensus regarding the radiation dose constraints for OARs in neuro-oncology based on the available literature. For the model-based comparison of photon and proton treatment plans as well as for prospective clinical trials, the use of these consented constraints is strongly encouraged ${ }^{57,58}$.

Chapter 7 systematically reviews the available evidence on the efficacy of high precision radiotherapy for drug-resistant non-neoplastic focal epilepsy in adults. By incorporating the available response data from literature, a dose effect-model was fitted to describe a relationship between dose and the seizure frequency reduction defined as the radiotherapy-adapted Engel class ${ }^{67}$.

In Chapter 8 the results of this thesis are discussed and summarised. A Dutch and English summary and reports on the valorisation of this thesis is presented in Chapter 9. 


\section{References}

1. Chakraborty S, Patil VM, Babu S, Muttath G, Thiagarajan SK. Locoregional recurrences after postoperative volumetric modulated arc radiotherapy (VMAT) in oral cavity cancers in a resource constrained setting: experience and lessons learned. Br J Radiol 2015;88:20140795.

2. Orlandi E, Giandini T, lannacone E, De Ponti E, Carrara M, Mongioj V, et al. Radiotherapy for unresectable sinonasal cancers: dosimetric comparison of intensity modulated radiation therapy with coplanar and non-coplanar volumetric modulated arc therapy. Radiother Oncol 2014;113:260-6.

3. Chakraborty S, Patil VM, Babu S, Muttath G, Thiagarajan SK. Locoregional recurrences after postoperative volumetric modulated arc radiotherapy (VMAT) in oral cavity cancers in a resource constrained setting: experience and lessons learned. Br J Radiol 2015;88:20140795.

4. Vanetti E, Clivio A, Nicolini G, Fogliata A, Ghosh-Laskar S, Agarwal JP et al. Volumetric modulated arc radiotherapy for carcinomas of the oro-pharynx, hypo-pharynx and larynx: a treatment planning comparison with fixed field IMRT. Radiother Oncol 2009;92:111-7.

5. Ferlay J, Soerjomataram I, Ervik M, Dikshit R, editors. IARC cancer base No. Lyon, France: International Agency for Research on Cancer; 2013.

6. Laramore GE, Scott CB, Al-Sarraf M, Haselow RE, Ervin TJ, Wheeler R, et al. Adjuvant chemotherapy for resectable squamous cell carcinomas of the head and neck: report on Intergroup Study 0034. Int J Radiat Oncol Biol Phys 1992;23:706-13.

7. Forastiere AA, Goepfert H, Maor M, Pajak TF, Weber R, Morrison W, et al. Concurrent chemotherapy radiotherapy for organ preservation in advanced laryngeal cancer. N Engl J Med 2003;349:2091-8.

8. Cooper JS, Pajak TF, Forastiere AA, Jacobs J, Campbell BH, Saxman SB, et al. Postoperative concurrent radiotherapy and chemotherapy for high-risk squamous-cell carcinoma of the head and neck. N Engl J Med 2004;350:1937-44.

9. Hoebers F, Heemsbergen W, Moor S, Lopez M, Klop M, Tesselaar M, Rasch C. Reirradiation for headand-neck cancer: delicate balance between effectiveness and toxicity. Int J Radiat Oncol Biol Phys 2011;81: e8-e111.

10. Lee N, Chan K, Bekelman JE, Mechalakos J, Narayana A, Wolden S, et al. Salvage re-irradiation for recurrent head and neck cancer. Int J Radiat Oncol Biol Phys 2007;68:731-40.

11. Langer CJ, Harris J, Horwitz EM, Nicolaou N, Kies M, Curran W, et al. Phase II study of low-dose paclitaxel and cisplatin in combination with split-course concomitant twice-daily reirradiation in recurrent squamous cell carcinoma of the head and neck: Results of Radiation Therapy Oncology Group Protocol 9911. J Clin Oncol 2007;25:4800-5.

12. Kramer NM, Horwitz EM, Cheng J, Ridge JA, Feigenberg SJ, Cohen RB, et al. Toxicity and outcome analysis of patients with recurrent head and neck cancer treated with hyperfractionated split-course reirradiation and concurrent cisplatin and paclitaxel chemotherapy from two prospective phase I and II studies. Head Neck 2005;27:406-14.

13. de Crevoisier R, Bourhis J, Domenge C, Wibault P, Koscielny S, Lusinchi A, et al. Full-dose reirradiation for unresectable head and neck carcinoma: experience at the Gustave-Roussy institute in a series of 169 patients. J Clin Oncol 1998;16:3556-62.

14. Wong S, Machtay M, Li Y. Locally recurrent, previously irradiated head and neck cancer: concurrent reirradiation and chemotherapy, or chemotherapy alone? J Clin Oncol 2006;24:2653-8.

15. Salama JK, Vokesa EE. Concurrent chemotherapy and re-irradiation for locoregionally recurrent head and neck cancer. Semin Oncol 2008;35:251-61.

16. Crocetti E, Trama A, Stiller C, Caldarella A, Soffietti R, Jaal J, et al. Epidemiology of glial and non-glial brain tumours in Europe. Eur J Cancer 2012;48:1532-42.

17. Louis DN, Perry A, Reifenberger G, von Deimling A, Figarella-Branger D, Cavenee WK, et al. The 2016 World Health Organization classification of tumours of the central nervous system: a summary. Acta Neuropathol 2016;131:803-20.

18. Eckel-Passow JE, Lachance DH, Molinaro AM, Walsh KM, Decker PA, Sicotte H, et al. Glioma Groups Based on 1p/19q, IDH, and TERT Promoter Mutations in Tumours. N Engl J Med 2015;372:2499-508. 
19. Brat DJ, Verhaak RG, Aldape KD, Yung WK, Salama SR, Cooper LA, et al. Cancer Genome Atlas Research Network. Comprehensive, Integrative Genomic Analysis of Diffuse Lower-Grade Gliomas. N Engl J Med 2015;372:2481-98.

20. Wesseling P, Capper D. WHO 2016 Classification of Gliomas. Neuropathol Appl Neurobiol 2018;44: 139-150.

21. Van der Vlis TAMB, Hoeben A, Beckervordersandforth JC, Ackermans L, Eekers DBP, Wennekes RMJ, et al. Impact of the revised WHO classification of diffuse low-grade glioma on clinical decision making: A case report. Surg Neurol Int 2017;8:223.

22. Buckner JC, Shaw EG, Pugh SL, Chakravarti A, Gilbert MR, Barger GR, et al. Radiation plus Procarbazine, CCNU, and Vincristine in Low-Grade Glioma. N Engl J Med 2016;374:1344-55.

23. Edelstein K, Richard NM, Bernstein L. Neurocognitive impact of cranial radiation in adults with cancer: an update of recent findings. Curr Opin Support Palliat Care 2017;11:32-37.

24. Greene-Schloesser D, Robbins ME. Radiation-induced cognitive impairment-from bench to bedside. Neuro-Oncol 2012;14 Suppl 4:iv37-44.

25. Gondi V, Hermann BP, Mehta MP, Tomé WA. Hippocampal dosimetry predicts neurocognitive function impairment after fractionated stereotactic radiotherapy for benign or low-grade adult brain tumours. Int J Radiat Oncol Biol Phys 2013;85:348-54

26. Rapoport $\mathrm{M}$, van Reekum $\mathrm{R}$, Mayberg $\mathrm{H}$. The role of the cerebellum in cognition and behavior: a selective review. J Neuropsychiatry Clin Neurosci 2000;12:193-8.

27. Leiner HC. Solving the mystery of the human cerebellum. Neuropsychol Rev 2010;20:229-35.

28. Stoodley CJ, Schmahmann JD. Evidence for topographic organization in the cerebellum of motor control versus cognitive and affective processing. Cortex J Devoted Study Nerv Syst Behav 2010;46:831-44.

29. Stoodley CJ, Valera EM, Schmahmann JD. Functional topography of the cerebellum for motor and cognitive tasks: an fMRI study. Neurolmage 2012;59:1560-70.

30. Kerkhof M, Vecht CJ. Seizure characteristics and prognostic factors of gliomas. Epilepsia 2013;54:12-7.

31. Pallud J, Audureau E, Blonski M, Sanai N, Bauchet L, Fontaine D, et al. Epileptic seizures in diffuse lowgrade gliomas in adults. Brain 2014;137:449-62.

32. Shin JY, Kizilbash SH, Robinson SI, Uhm JH, Hammack JE, Lachance DH, et al. Seizures in patients with primary brain tumours: what is their psychosocial impact? J Neurooncol 2016;128:285-91.

33. Banerjee PN, Filippi D, Allen Hauser W. The descriptive epidemiology of epilepsy-a review. Epilepsy Res 2009;85:31-45.

34. Rocha L. Interaction between electrical modulation of the brain and pharmacotherapy to control pharmacoresistant epilepsy. Pharmacol Ther 2013;138:211-228.

35. World Health Organization. The World Health Report 2001: Mental Health, New Understanding New Hope. Geneva: World Health Organization 2001;178.

36. Fisher R, van Emde Boas W, Blume W, Elger C, Genton P, Lee P, Engel J. Epileptic seizures and epilepsy: definitions proposed by the International League Against Epilepsy (ILAE) and the International Bureau for Epilepsy (IBE). Epilepsia 2005;46:470-2.

37. Rudà R, Magliola U, Bertero U, Trevisan E, Bosa C et al. Seizure control following radiotherapy in patients with diffuse gliomas: a retrospective study Neuro Oncol 2013;15:1739-49.

38. Berg AT, Berkovic SF, Brodie MJ, Buchhalter J, Cross JH, van Emde Boas W, et al. Revised terminology and concepts for organization of seizures and epilepsies: report of the ILAE Commission on Classification and Terminology, 2005-2009. Epilepsia 2010;51:676-85.

39. Blumcke I, Spreafico R, Haaker G, Coras R, Kobow K, Bien CG, et al. Histopathological Findings in Brain Tissue Obtained during Epilepsy Surgery. EEBB Consortium. New Engl J Med 2017;377:1648-56.

40. Régis J. Gamma knife for functional diseases. Neurotherapeutics 2014;11:583-92.

41. Quigg M, Harden C. Minimally invasive techniques for epilepsy surgery: stereotactic radiosurgery and other technologies. J Neurosurg 2014;121:232-40

42. Uijl SG, Moons KG, Leijten FS et al. Is epilepsy surgery utilized to its full extent? Epilepsia 2008;49: 1480-1.

43. Régis J, Carron R, Park M. Is radiosurgery a neuromodulation therapy? A 2009 fabrikant award lecture. J Neurooncol 2010;98:155-62. 
44. Choi H, Sell R, Lenert L, Muennig P, Goodman RR, Gilliam FG, Wong JB. Epilepsy surgery for pharmacoresistant temporal lobe epilepsy: a decision analysis. JAMA 2008;300:497-505.

45. Langendijk JA, Lambin P, De Ruysscher D, Widder J, Bos M, Verheij M. Selection of patients for radiotherapy with protons aiming at reduction of side effects: The model-based approach. 2013;107:3:267-73.

46. van Dijk LV, Steenbakkers RJHM, ten Haken B, van der Laan HP, van 't Veld AA, Langendijk JA, Korevaar EW. et al. Robust Intensity Modulated Proton Therapy (IMPT) Increases Estimated Clinical Benefit in Head and Neck Cancer Patients. PLoS One 2016;11:e0152477.

47. Eekers DBP, Erik Roelofs E, Jelen U, Kirk M, Granzier M, Ammazzalorso F, et al. Benefit of particle therapy in re-irradiation of head and neck patients. Results of a multicenter in silico ROCOCO trial. Cancer Data 2016. Radiother Oncol 2016;121:387-94.

48. van der Laan HP, van de Water TA, van Herpt HE, Christianen ME, Bijl HP, Korevaar EW, Rasch CR, van 't Veld AA, van der Schaaf A, Schilstra C, Langendijk JA; Rococo cooperative group. The potential of intensity-modulated proton radiotherapy to reduce swallowing dysfunction in the treatment of head and neck cancer: A planning comparative study. Acta Oncol 2013;52:561-9.

49. Rosenblatt E, Izewska J, Anacak Y, Pynda Y, Scalliet P, Boniol M, Autier P. Radiotherapy capacity in European countries: an analysis of the Directory of Radiotherapy Centres (DIRAC) database.Lancet Oncol 2013;14:e79-86.

50. Beetz I, Schilstra C, van der Schaaf A, van den Heuvel ER, Doornaert P, van Luijk P, Vissink A et al. NTCP models for patient-rated xerostomia and sticky saliva after treatment with intensity modulated radiotherapy for head and neck cancer: the role of dosimetric and clinical factors. Radiother Oncol 2012;105:101-6.

51. Dijkema T, Terhaard CH, Roesink JM, Braam PM, van Gils CH, Moerland MA, Raaijmakers CP. Large cohort dose-volume response analysis of parotid gland function after radiotherapy: intensitymodulated versus conventional radiotherapy. Int J Radiat Oncol Biol Phys 2008;15;72:1101-9.

52. Dijkema T, Raaijmakers CP, Ten Haken RK, Roesink JM, Braam PM, Houweling AC et al. Parotid gland function after radiotherapy: the combined Michigan and Utrecht experience. Int J Radiat Oncol Biol Phys 2010;78:449-53.

53. Christianen ME, Schilstra C, Beetz I, Muijs CT, Chouvalova O, Burlage FR et al. Predictive modelling for swallowing dysfunction after primary (chemo)radiation: results of a prospective observational study. Radiother Oncol 2012;105:107-14.

54. Brouwer CL, Steenbakkers RJ, Bourhis J, Budach W, Grau C, Grégoire V, et al. CT-based delineation of organs at risk in the head and neck region: DAHANCA, EORTC, GORTEC, HKNPCSG, NCIC CTG, NCRI, NRG Oncology and TROG consensus guidelines. Radiother Oncol 2015;117:83-90.

55. Eekers DBP, in 't Ven L, Roelofs E, Postma A, Alapetite C, Burnet N, et al. The EPTN consensus-based atlas for CT- and MR-based contouring in Neuro-Oncology. "European Particle Therapy Network" of ESTRO. Radiother Oncol 2018. pii: S0167-8140(17)32768-8.

56. Eekers DBP, in 't Ven L, Roelofs E, Postma A, Troost EGC. EPTN International Neurological Contouring Atlas. CancerData 2017. pii/S2405630817300769.

57. Lambrecht M, Eekers DBP, Alapetite C, Burnet NG, Calugaru V et al. Radiation dose constraints for organs at risk in neuro-oncology; the European Particle Therapy Network consensus. Radiother Oncol 2018; 128(1):26-36.

58. Eekers DBP, Lambrecht M, Nyström PDW, Swinnen A, Wesseling FWR, Roelofs E, Troost EG. EPTN consensus-based guideline for the tolerance dose per fraction of organs at risk in the brain. CancerData, 2018; pii/S0167814013004350.

59. Emami B, Lyman J, Brown A, Coia L, Goitein M, Munzenrider JE, et al. Tolerance of normal tissue to therapeutic irradiation. Int J Radiat Oncol Biol Phys 1991;21:109-22.

60. Marks LB, Yorke ED, Jackson A, Ten Haken RK, Constine LS, Eisbruch A, et al. Use of normal tissue complication probability models in the clinic. Int J Radiat Oncol Biol Phys 2010;76:S10-9.

61. Mayo C, Martel MK, Marks LB, Flickinger J, Nam J, Kirkpatrick J. Radiation Dose-Volume Effects of Optic Nerves and Chiasm. Int J Radiat Oncol 2010;76:S28-35.

62. Mayo C, Yorke E, Merchant TE. Radiation associated brainstem injury. Int J Radiat Oncol Biol Phys 2010;76:S36-41. 
63. Lawrence YR, Li XA, el Naqa I, Hahn CA, Marks LB, Merchant TE, et al. Radiation dose-volume effects in the brain. Int J Radiat Oncol Biol Phys 2010;76:S20-7.

64. Roelofs E, Engelsman M, Rasch C, Persoon L, Qamhiyeh S, de Ruysscher D, et al. ROCOCO Consortium. Results of a multicentric in silico clinical trial (ROCOCO): comparing radiotherapy with photons and protons for non-small cell lung cancer. J Thorac Oncol 2012;7:165-76.

65. Eekers DBP, Roelofs E, Jelen U, Kirk M, Granzier M, Ammazzalorso F, et al. Data from: Benefit of particle therapy in re-irradiation of head and neck patients. Results of a multicenter in silico ROCOCO trial. 2016. pii: S0360-3016:26569-8.

66. van der Laan HP, van de Water TA, van Herpt HE, Christianen ME, Bijl HP, Korevaar EW, et al; Rococo cooperative group. The potential of intensity-modulated proton radiotherapy to reduce swallowing dysfunction in the treatment of head and neck cancer: A planning comparative study. Acta Oncol 2013;52:561-9.

67. Eekers DBP, Pijnappel EN, Schijns OEMG, Colon A, Hoeben A, Zindler et al. Evidence on the efficacy of primary radiosurgery or stereotactic radiotherapy for drug-resistant non-neoplastic focal epilepsy in adults: a systematic review. Seizure 2018;55:83-92.

68. Eekers DBP, Roelofs E, Cubillos-Mesias M, Niel, C, Smeenk RJ, Hoeben A et al. Intensity-modulated proton therapy decreases dose to organs at risk in low-grade glioma patients: results of a multicentric in silico ROCOCO trial. In press Acta Oncol 2018.

69. Eekers DBP, In 't Ven L, Deprez S, Jacobi L, Roelofs E, Hoeben A et al. The posterior cerebellum, a new organ at risk? Clin Transl Radiat Oncol 2017;8:22-26. 


\section{Chapter 2

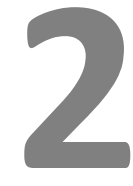

\section{Benefit of particle therapy in re-irradiation \\ of head and neck patients. Results of a \\ multicentric in silico ROCOCO trial}

Daniëlle B.P. Eekers, Erik Roelofs, Urszula Jelen, Maura Kirk, Marlies Granzier, Filippo Ammazzalorso, Peter H. Ahn, Geert O.R.J Janssens, Frank J.P. Hoebers, Tobias Friedmann, Timothy Solberg, Sean Walsh, Esther G.C. Troost, Johannes H.A.M. Kaanders, Philippe Lambin

Radiother Oncol. 2016;121(3):387-394 


\section{Abstract}

\section{Background and purpose}

In this multicentric in silico trial we compared photon, proton, and carbon-ion radiotherapy plans for re-irradiation of patients with squamous cell carcinoma of the head and neck (HNSCC) regarding dose to tumour and doses to surrounding organs at risk (OARs).

\section{Material and methods}

Twenty-five HNSCC patients with a second new or recurrent cancer after previous irradiation (70 Gy) were included. Intensity-modulated proton therapy (IMPT) and ion therapy (IMIT) re-irradiation plans to a second subsequent dose of 70 Gy were compared to photon therapy delivered with volumetric modulated arc therapy (VMAT).

\section{Results}

When comparing IMIT and IMPT to VMAT, the mean dose to all investigated 22 OARs was significantly reduced for IMIT and to 15 out of 22 OARs (68\%) using IMPT. The maximum dose to $2 \%$ volume $\left(D_{2}\right)$ of the brainstem and spinal cord were significantly reduced using IMPT and IMIT compared to VMAT. The data are available on www.cancerdata.org.

\section{Conclusions}

In this ROCOCO in silico trial, a reduction in mean dose to OARs was achieved using particle therapy compared to photons in the re-irradiation of HNSCC. There was a dosimetric benefit favouring carbon-ions above proton therapy. These dose reductions may potentially translate into lower severe complication rates related to the reirradiation. 


\section{Introduction}

Squamous cell carcinoma of the head and neck (HNSCC) is the sixth most prevalent cancer in the world and is still considered difficult to cure ${ }^{1,2}$. In addition to the abuse of alcohol and/or tobacco, infection with human papilloma virus (HPV) is a risk factor.

Despite many new treatment options, the risk of local or regional recurrence for patients with HNSCC having undergone (chemo)radiotherapy or tri-modality treatment is still up to $30 \%^{3-5}$. Additionally, the estimated 5-year cumulative incidence of second new tumours was $13 \%$ in the radiotherapy group and $12 \%$ in the combined therapy group according to Bernier et al. ${ }^{6}$. The treatment of locoregional recurrence of HNSCC consists of surgical resection with or without adjuvant (chemo)radiation or primary definitive (chemo)radiation, leading to an overall 5-year relative survival rate of $40-66 \%$ in selected patients ${ }^{1}$.

In case of an unresectable recurrence, re-irradiation (re-RT) possibly combined with systemic therapy, is an alternative curative option. In the postoperative setting, re-RT should be considered if features in the pathology specimen, such a positive resection margin or extracapsular extension, indicate high risk of recurrence ${ }^{7-10}$. Long-term disease control and survival can be achieved in patients who receive re-RT as an adjunct to surgical resection. However, the rates of severe grade 3 or 4 toxicity after re-RT are high, with an incidence of approximately $45 \%$ at 5 years, and only approximately 1 in 3 patients survives re-RT without recurrence and severe complications ${ }^{11}$. Reduction of the risk of radiation-induced complications and improvement of the oncological outcome are needed ${ }^{8,12-17}$.

In recent years, there has been enormous progress in radiotherapy techniques, moving from 3D conformal radiotherapy (3D-CRT) to intensity-modulated radiotherapy (IMRT) and volumetric modulated arc therapy (VMAT), enabling highly conformal treatment with dose reductions to healthy tissue and surrounding organs at risk (OARs) ${ }^{18,19}$. Still the OARs and healthy tissues frequently receive a significant dose with 3D-CRT and IMRT $^{19,20}$.

Particle therapy (PT) is becoming increasingly available, which prompts interest among physicians as well as health insurance companies regarding its efficacy in HNSCC. To assess the potential gains of PT for individual patients in the re-irradiation setting, we conducted an in silico treatment planning study on a cohort of 25 HNSCC patients retrospectively retrieved from two Dutch radiotherapy departments. Data are available on www.cancerdata.org ${ }^{21}$. 


\section{Methods}

\section{Study population}

We retrospectively retrieved treatment plans of HNSCC patients stage I to IVB who had undergone (chemo)radiotherapy with curative intent ( $\geq 50 \mathrm{~Gy}$, including at least lymph node levels II-IV) at Radboud University Medical Centre (UMC) or at MAASTRO clinic and who were subsequently (>1 year later) re-irradiated (with or without surgery and/or chemotherapy), with a relevant overlap of the target volume (clinicaltrial.gov ID: NCT 02242916).

\section{Target volume and OAR definition}

An individual neck support and head fixation was used in all patients. For treatment planning purposes, computed tomography (CT) images with intravenous contrast were used. The gross tumour volume (GTV) was delineated as the macroscopic tumour on the planning-CT fused with the hybrid fluorodeoxyglucose positron emission tomography (FDG-PET) and/or after rigid mutual-information-based registration with magnetic resonance imaging (MRI). The GTV and the clinical target volume (CTV) were delineated using local guidelines (RadboudUMC or MAASTRO clinic) based on Gregoire et al. ${ }^{22-24}$. The CTV was expanded to the planning target volume (PTV) taking into account the individual institutions' margin recipe: VMAT utilized $4 \mathrm{~mm}$ accounting for setup errors, intensity-modulated ion therapy (IMIT) employed $4 \mathrm{~mm}$ and intensitymodulated proton therapy (IMPT) $5 \mathrm{~mm}$ and $3 \mathrm{~mm}$ for the boost to the high risk PTV, to account for both setup and range uncertainties. The low risk volume, PTV with a prescribed dose of 54 Gy $\left(\mathrm{PTV}_{54 \mathrm{~Gy}}\right)$, consisted of the tumour or tumour bed, the pathological lymph nodes and the elective lymph node levels. The high risk PTV planned to a total dose of 70 Gy (PTV 70 Gy $)$ was defined as the tumour or tumour bed and/or pathological lymph nodes. For all 25 patients included in this in silico study, the OARs were outlined by one dosimetrist (M.G.) and supervised by a radiation oncologist (D.E.) for the first and re-RT study set (see Table 2.1 for the list of OARs). Dental fillings and associated artefacts were delineated and the density was overridden to that of teeth or tissue, respectively, for dental fillings within the treatment beam were an exclusion criterion for PT. The use of any form of bolus to assure an accurate coverage of the target volume was permitted. There was no correction for the use of intravenous contrast. 
Table 2.1 Dose constraints and priorities for organs at risk. The tolerance dose per OAR for the first and second treatment in Gy (E) with their planning priority.

\begin{tabular}{|c|c|c|c|}
\hline \multirow[b]{2}{*}{ OAR } & First treatment & \multicolumn{2}{|c|}{ Second treatment } \\
\hline & Tolerance & Tolerance & Priority \\
\hline Brain Stem & $D_{\max } 54 \mathrm{~Gy}$ & individual* & 1 \\
\hline Spinal cord & $D_{\max } 50 \mathrm{~Gy}$ & individual* & 1 \\
\hline Larynx & $D_{\max } 45 \mathrm{~Gy}$ & $120 \mathrm{~Gy}^{* *}$ & 1 \\
\hline Arytenoid & $D_{\max } 50 \mathrm{~Gy}$ & $100 \mathrm{~Gy}^{* *}$ & 1 \\
\hline Mandible & $D_{\max } 60 \mathrm{~Gy}$ & $120 \mathrm{~Gy}^{* *}$ & 1 \\
\hline Parotid glands (spared) & $D_{\text {mean }} 26 \mathrm{~Gy}$ & no limit & 2 \\
\hline Submandibular glands (spared) & $\mathrm{D}_{\text {mean }} 26 \mathrm{~Gy}$ & no limit & 3 \\
\hline Swallowing muscle & $D_{\max } 50 \mathrm{~Gy}$ & no limit & 4 \\
\hline Carotid artery & $D_{\max } 50 \mathrm{~Gy}$ & no limit & 5 \\
\hline Jugular artery & $D_{\max } 50 \mathrm{~Gy}$ & no limit & 5 \\
\hline Oral cavity & $\mathrm{D}_{\text {mean }} 26 \mathrm{~Gy}$ & no limit & 5 \\
\hline Sternocleidomastoid muscle & $D_{\max } 60 \mathrm{~Gy}$ & no limit & 5 \\
\hline Thyroid & $D_{\max } 60 \mathrm{~Gy}$ & no limit & 5 \\
\hline Vertebrae & $D_{\max } 60 \mathrm{~Gy}$ & no limit & 5 \\
\hline
\end{tabular}

Dose limiting, exceeding this dose is not permitted

Only dose limiting if the OAR was not part of the CTV in the first and second treatment

Kept as low as possible if OAR was spared in the first treatment, not dose limiting

Goal limits, not dose limiting

* For each individual patient calculated assuming 30\% recovery from first treatment

** Cumulative dose, the sum of the first and second treatment

OAR=organs at risk, $D_{\max }=$ maximum dose, $D_{\text {mean }}=$ mean dose.

\section{Treatment planning}

The first treatment was considered a precondition in all patients, determining the remaining tolerance dose for the OARs, and therefore not described here. All subsequent paragraphs relate to the re-RT treatment plans. The PTV F4Gy $_{\text {was }}$ irradiated to a total dose of $54.25 \mathrm{~Gy}$ in 35 fractions of $1.55 \mathrm{~Gy}$, the PTV $\mathrm{P}_{70 \mathrm{~Gy}}$ to $70 \mathrm{~Gy}$ in 35 fractions of $2 \mathrm{~Gy}$ using a simultaneous integrated boost (SIB) technique. In case no elective lymph nodes were included, 70 Gy was prescribed in 35 fractions of 2 Gy to PTV 70Gy $_{\text {(the }}$ tumour or pathological lymph node only).

All proton and photon treatment plans were calculated in centres that were already operating and had significant clinical experience treatment planning. The prescribed dose to the PTV 70 Gy was set to 70 Gy of which $99 \%$ of the volume had to be covered by $95 \%$ of the dose for all modalities in order to enable a direct comparison. All plans were evaluated for robustness. 


\section{Dose constraints}

For the OARs, the dose limits and priorities were defined in the protocol (Table 2.1). The dose-volume histograms (DVHs) of the original treatment plans were used to calculate the re-RT constraints for four OARs: the brainstem, spinal cord, mandible and larynx (including arytenoid). Since there was an interval of $\geq 1$ year between first and second treatment, a 30\% recovery was assumed for these four OARs ${ }^{36-38}$. The locations of the maximum dose $\left(D_{\max }\right)$ within an OAR in the first and secondary treatment plans were assumed to be the same, as a worst-case scenario, since the average overlap of the second treatment volume with the first treatment volume was determined to be $67 \%$. No correction for fraction size was performed. Parotid and submandibular glands, if preserved in the first treatment, were attempted to be spared with second and third priority, respectively, without being dose limiting. For larynx, arytenoids and mandible, cumulative dose, which was the sum of the first and second treatment, was considered only dose limiting if the OAR was not part of the CTV in the first and second treatment (see Table 2.1). Attempts were made to minimize dose to the base of tongue, carotid arteries, jugular veins, oral cavity, sternocleidomastoid muscles, swallowing muscles, thyroid and vertebrae, but these structures were not dose limiting. For each re-RT plan, the mean dose $\left(D_{\text {mean }}\right)$ was calculated per OAR as well as the near-maximum dose, defined as the highest dose to $2 \%$ of the volume $\left(D_{2}\right)$, and the near-minimum dose defined as the lowest dose to $98 \%$ of the volume $\left(D_{98}\right)^{39}$. The mean integral dose (ID) was defined as the mean dose to the imaged part of the patient (body contour) minus CTV, also known as the residual volume at risk (RVR).

\section{Photons}

The original clinically applied re-RT treatment plans were created at RadboudUMC using Pinnacle (Pinnacle v8.2g Philips, WI) and at MAASTRO clinic using Varian (Eclipse ${ }^{\mathrm{TM}}$ v11.0 Varian Medical Systems, Palo Alto, CA). In order to account for improvements in photon plan techniques over the past years, the actual re-RT photon treatment plans were re-calculated employing state-of-the-art VMAT planning at MAASTRO clinic. With regard to VMAT, photons were considered to be innately robust relative to PT, therefore no explicit optimization techniques were incorporated into the VMAT plans as the PTV was assumed to be sufficient to produce a robust plan.

\section{Protons}

Proton re-treatment plans were calculated at University of Pennsylvania (UPENN) using IMPT for beam delivery with pencil beam scanning (PBS) (Eclipse ${ }^{\mathrm{TM}}$ v11.0 Varian Medical 
Systems) using beam data modelled for IBA universal nozzle on a gantry (Ion Beam Applications, Louvain-la-Neuve, Belgium) ${ }^{25}$. Multi-field optimization (MFO) was used in the planning process as it produced superior OAR sparing compared to a single field uniform dose (SFO) approach ${ }^{26}$. To preserve small spots, UPENN utilised a universal bolus instead of a more conventional range shifter for the treatment of head and neck tumours $^{27}$. A relative biological effectiveness (RBE) of 1.1 was used ${ }^{28}$. A typical field arrangement consisted of two posterior oblique beams or a posterior and posterior oblique beam, depending on target geometry. A lateral field was added or substituted for one posterior oblique when the $D_{\max }$ spinal cord constraint could not be met due to high dose target structures crossing the midline distal to the cord. PT is, with regard to IMPT, known to be intrinsically non-robust, therefore a PTV with a margin of $3 \mathrm{~mm}$ from the CTV was created with an additional $2 \mathrm{~mm}$ added to the planning optimization volume to account for range uncertainty. The $5 \mathrm{~mm}$ margin is a conservative value for head and neck assuming an overall uncertainty of $3.5 \%$ in conversion from Hounsfield Unit (HU) to relative proton stopping power and a range of approximately $15 \mathrm{~cm}^{29,30}$. The following margin definition in head and head-and-neck boost treatments was used: $3 \mathrm{~mm}$ transversal to the beam (on account of the residual patient positioning error, robotic table/imager uncertainties and beam steering) and $3 \mathrm{~mm}$ along the beam on account of the range uncertainties assuming $\mathrm{HU}$ calibration accuracy of $3 \%$ and beam range of ca. $10 \mathrm{~cm}^{31,32}$.

\section{Carbon ions}

Carbon ion (C-ion) re-RT treatment plans were calculated at University of Marburg using a raster scanning technique with intensity-modulated ion therapy (IMIT) using Syngo PT Planning (Siemens Health Care Systems, Erlangen, Germany), which employed the local effect model (LEM1) for the biologically weighted dose computation ${ }^{33,34}$. The number of fields varied from 1 (in 1 case) to 3 (in 3 cases). The beam directions (using isocentric table rotations and gantry when deemed advantageous) were chosen individually for each case with two main considerations: avoidance of any unnecessary dose and evading strong density heterogeneities in the beam entrance channels ${ }^{35}$. Furthermore, beam directions traversing patient support and immobilization devices were avoided. For multiple beam setups reduced the under-dosages caused by potential range uncertainties, a uniform margin of $4 \mathrm{~mm}$ was applied with a tolerance of $2 \mathrm{~mm}$, allowing the TPS to place additional raster spots outside the PTV as necessary, thus ensuring target coverage. 


\section{Plan robustness evaluation}

All treatment plans (VMAT, IMPT, and IMIT) were assessed for robustness of the CTV coverage in their respective centres of origin. The robustness of the treatment plans was evaluated using worst-case scenario plan calculations with a $\pm 3 \mathrm{~mm}$ isocenter shift in $x_{-}, y_{-}$, and $z$-directions combined with a $\pm 3.5 \%$ density shift (12 combinations per plan). The minimal dose to $95 \%$ and $98 \%$ of the CTV is given ( $D_{95}$ and $\left.D_{98}\right)$, respectively.

\section{Storage of imaging datasets}

The datasets was stored and exchanged through the secured collaborative MISTIR platform (www.mistir.info) hosted by MAASTRO clinic. Quality assurance procedures were applied to assess the necessity of corrections of transformations during treatment planning system (TPS) import and export ${ }^{40}$.

\section{Data evaluation and statistical analysis}

Matlab software (The Math Works, Natick, MA) was used for statistical analysis. The dose matrices were scaled to the mean CTV $_{70 G y}$ doses of 70 Gy (RBE equivalent) as this structure did not change between modalities. The dose metrics were extracted from the plan and statistically compared using two-tailed, signed-rank Wilcoxon tests to determine the significance of pairwise differences compared to VMAT (a p-value of $<0.02$ was considered significant, taking a Bonferroni correction into account).

\section{Results}

Twenty-five cases were included and in total 75 re-RT plans were analysed; one example is illustrated in Figure 2.1. All treatment modalities achieved a comparable dose to the $\mathrm{CTV}_{54 \mathrm{~Gy}}$ and $\mathrm{CTV}_{70 \mathrm{~Gy}}$ (Table 2.2). All plans were judged to be clinically applicable with worst-case scenario CTV robustness tests resulting in $D_{98}=95.4 \pm 1.9 \%$ and $D_{95}=97.9 \pm 1.4 \%$ for VMAT, $D_{98}=94.6 \pm 2.8 \%$ and $D_{95}=96.2 \pm 2.4 \%$ for IMPT, and $\mathrm{D}_{98}=93.1 \pm 3.0$ and $\mathrm{D}_{95}=96.1 \pm 1.8$ for IMIT (see Supplementary Table S2.1).

The average scaled values of the $D_{\text {mean }}$ and $D_{2}$ for the OARs were compared between VMAT and IMPT/IMIT (Table 2.2, dose significance indicated with an asterisk). When comparing IMIT and IMPT to VMAT, the $D_{\text {mean }}$ to all 22 OARs was statistically significantly reduced for IMIT and to 15 out of 22 OARs $(68 \%)$ using IMPT $(p<0.02)$. The $D_{2}$ for the brainstem and spinal cord were statistically significantly reduced $(p<0.02)$ 
using IMPT and IMIT compared to VMAT. The integral dose was highest for VMAT (5.9 Gy), followed by IMPT (3.9 Gy) and IMIT (2.7 Gy).

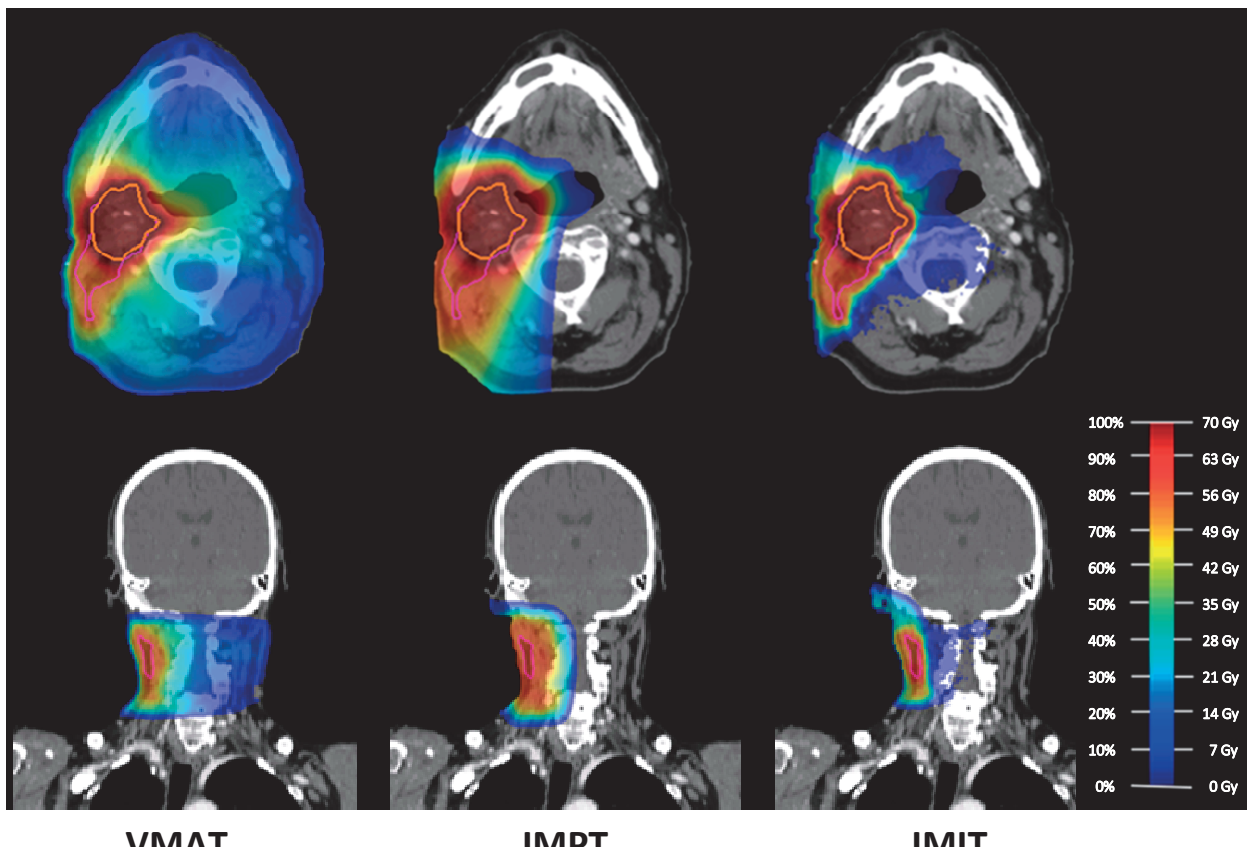

Figure 2.1 A set of re-irradiation treatment plans for a patient with a nodal recurrence of oropharynx carcinoma of the right tonsillar fossa, $\mathrm{rcT}_{0} \mathrm{~N}_{2 \mathrm{a}} \mathrm{M}_{0}$. In the VMAT (left column), IMPT (middle column) and IMIT (right column) plans, the $\mathrm{CTV}_{54 \mathrm{~Gy}}$ (pink) and high-dose $\mathrm{CTV}_{70 \mathrm{~Gy}}$ (orange). The dose-range is presented from high (red) to low (blue).

IMPT and IMIT proved to be superior to VMAT in sparing the contralateral located OARs. Depending on the OAR, this dose reduction was up to $85 \%-100 \%$ for the contralateral carotid artery and parotid gland, respectively, when using IMPT and 94\%99\% for the contralateral carotid artery and parotid gland, respectively, when using IMIT. Conversely, IMPT increased the ipsilateral dose to the parotid gland (by $1 \%$ ), carotid artery (by $2 \%$ ) and submandibular gland (by $1 \%$ ), albeit not to a clinically relevant level. The overall average $D_{\text {mean }}$ and $D_{2}$ for all OARs and each treatment modality were plotted in Figure 2.2, showing that IMIT resulted in a lower $D_{\text {mean }}$ in $100 \%$ of the cases and IMPT decreased the $D_{\text {mean }}$ in $86 \%$ of the OARs compared to VMAT. For the $D_{2}$, the respective numbers were $100 \%$ and $59 \%$. 
Table 2.2 Mean doses (SD) of selected dosimetric parameters for organs at risk and target volume in $\mathrm{Gy}(\mathrm{E})$ for IMPT and IMIT compared to VMAT after scaling to the mean CTV 70 Gy doses of $70 \mathrm{~Gy}$; * is significant $(p<0.02)$.

\begin{tabular}{|c|c|c|c|}
\hline$D_{\text {mean }}(G y)$ & VMAT & IMPT & IMIT \\
\hline Arytenoid ipsi \& bilateral & $34.5(24.4)$ & $27.3(24.9)$ & $20.6(24.9)^{*}$ \\
\hline Arytenoid contralateral & $17.6(13.2)$ & $2.3(4.2)^{*}$ & $1.1(0.9)^{*}$ \\
\hline Base of tongue & $32.1(19.4)$ & $25.5(23.3)$ & $18.8(21.7)^{*}$ \\
\hline Carotid ipsi- \& bilateral & $35.0(17.1)$ & $35.9(19.4)$ & $31.9(19.8)^{*}$ \\
\hline Carotid contralateral & $12.9(8.4)$ & $2.0(4.5)^{*}$ & $0.83(1.4)^{*}$ \\
\hline Body & $5.9(2.8)$ & $3.9(2.1)^{*}$ & $2.7(1.5)^{*}$ \\
\hline Jugular ipsi- \& bilateral & $29.6(20.2)$ & $29.0(22.5)$ & $24.6(22.8)^{*}$ \\
\hline Jugular contralateral & $10.4(6.6)$ & $0.97(3.0)^{*}$ & $0.57(1.6)^{*}$ \\
\hline Larynx & $34.1(18.0)$ & $27.2(18.4)^{*}$ & $20.3(17.5)^{*}$ \\
\hline Mandible & $16.1(12.7)$ & $11.5(11.5)^{*}$ & $8.2(11.0)^{*}$ \\
\hline Oral cavity & $14.9(14.3)$ & $9.0(13.7)^{*}$ & $7.6(14.6)^{*}$ \\
\hline Parotid ipsi- \& bilateral & $16.0(15.2)$ & $16.3(16.6)$ & $13.5(14.5)^{*}$ \\
\hline Parotid contralateral & $4.4(2.1)$ & $<0.01(0.02)^{*}$ & $0.038(0.13)^{*}$ \\
\hline Sterno cleido mastoid ipsi- \& bilateral & $31.2(17.1)$ & $30.9(20.5)$ & $26.3(19.5)^{*}$ \\
\hline Sterno cleido mastoid contralateral & $11.4(6.8)$ & $1.6(3.0)^{*}$ & $0.64(1.4)^{*}$ \\
\hline Submandibular gland ipsi- \& bilateral & $35.4(20.0)$ & $35.9(20.2)$ & $29.2(18.5)^{*}$ \\
\hline Submandibular gland contralateral & $16.3(9.3)$ & $0.64(1.7)^{*}$ & $0.73(1.2)^{*}$ \\
\hline Swallowing muscle total & $31.9(21.5)$ & $25.1(21.5)^{*}$ & $18.9(21.1)^{*}$ \\
\hline Thyroid & $30.9(25.2)$ & $29.8(25.2)$ & $25.9(24.4)^{*}$ \\
\hline Vertebrae & $18.1(7.6)$ & $10.8(6.8)^{*}$ & $5.8(4.2)^{*}$ \\
\hline CTV $_{54 G y}$ & $60.9(2.7)$ & $61.7(2.7)^{*}$ & $61.3(2.5)$ \\
\hline $\mathrm{CTV}_{70 G \mathrm{y}}$ & $70.0(<0.01)$ & $70.0(<0.01)$ & $70.0(<0.01)$ \\
\hline$D_{2}$ (Gy) & VMAT & IMPT & IMIT \\
\hline Brainstem & $8.2(7.9)$ & $2.7(5.3)^{*}$ & $1.3(2.5)^{*}$ \\
\hline Spinal cord & $16.6(4.7)$ & $6.7(5.7)^{*}$ & $4.8(3.2)^{*}$ \\
\hline $\mathrm{V}_{95}(\%)$ & VMAT & IMPT & IMIT \\
\hline CTV $_{54 G y}$ & $99.5(0.6)$ & $99.7(0.4)$ & $100.0(0.02)^{*}$ \\
\hline $\mathrm{CTV}_{70 \mathrm{~Gy}}$ & $98.9(2.5)$ & $99.9(0.3)$ & $99.9(0.2)$ \\
\hline
\end{tabular}

Dosimetric metrics of selected OARs per patient are plotted in Supplementary Figure S2.1 and S2.2. The maximum dose to the spinal cord $\left(D_{2}\right)$, was significantly reduced (Table 2.2) for most patients using IMPT (96\%) and for all patients using IMIT (100\%) (Supplementary Figure S2.2). Regarding $D_{\text {mean }}$ to the oral cavity and $D_{2}$ of the brainstem, IMIT decreased doses in all cases, compared to $88 \%$ and $96 \%$ of the cases when delivering IMPT. The $\mathrm{D}_{2}$ of the Larynx and mandible showed less benefit for IMPT and IMIT ( $28 \%$ and $28 \%$, and $50 \%$ and $76 \%$, respectively). For all modalities, radiation doses to the spinal cord and brainstem remained below the constraints without the need to underdose the targets (Supplementary Figure S2.1). For the mandible 32\% (VMAT), 36\% (IMPT) and 28\% (IMIT) of patients exceeded the cumulative dose of 120 Gy and the cumulative larynx constraint was not met in $78 \%, 83 \%$ and $83 \%$ of the patients, respectively. 

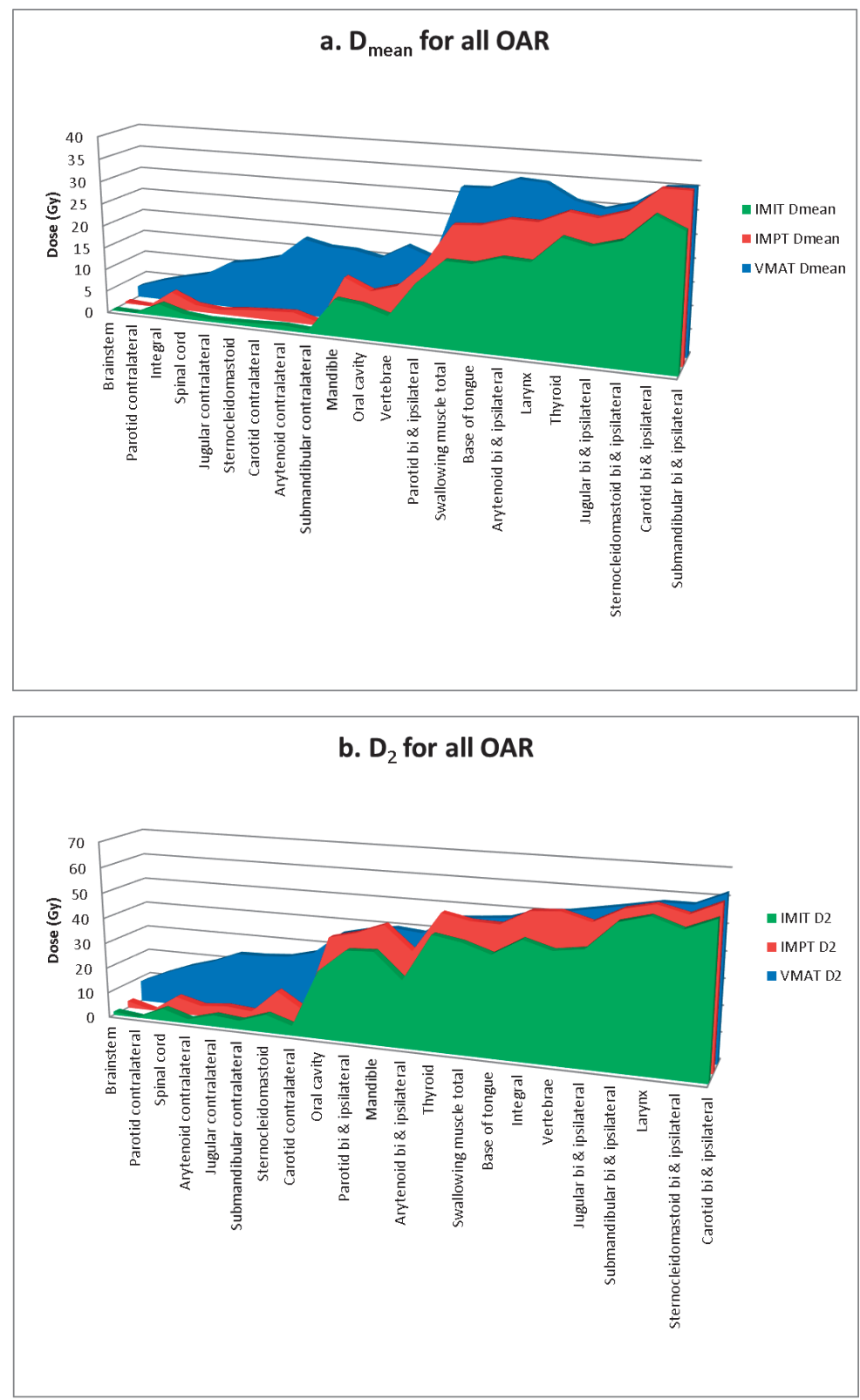

Figure 2.2 The overall average mean dose [ $\left.\left.D_{\text {mean; }} \mathrm{a}\right)\right]$ and maximum dose in Gy (equivalent) to $\left.2 \%\left[\mathrm{D}_{2}, \mathrm{~b}\right)\right]$ for all OARs is given for volumetric modulated arc therapy (VMAT, blue), intensity-modulated proton therapy (IMPT, red) and intensity-modulated ion therapy (IMIT, green). 
The number of patients that benefit from IMPT and/or IMIT over VMAT was plotted in Figure 2.3. All individual patients benefitted from IMIT with regard to arytenoid, oral cavity, spinal cord, brain stem and integral dose, as opposed to $80 \%-100 \%$ of the patients for IMPT. The $D_{2}$ to the bony structures (mandible and vertebrae) decreased in a low percentage of patients using IMPT (28\% and 16\%) as compared to a high percentage of patients using IMIT (76\% and 84\%). Overall, in this population of reirradiated HNSCC patients, PT generated a lower OAR dosage with an advantage of IMIT over IMPT compared to VMAT ( $84 \%$ and $60 \%$ respectively).

\section{Patients profit from IMPT \& IMIT compared to VMAT}

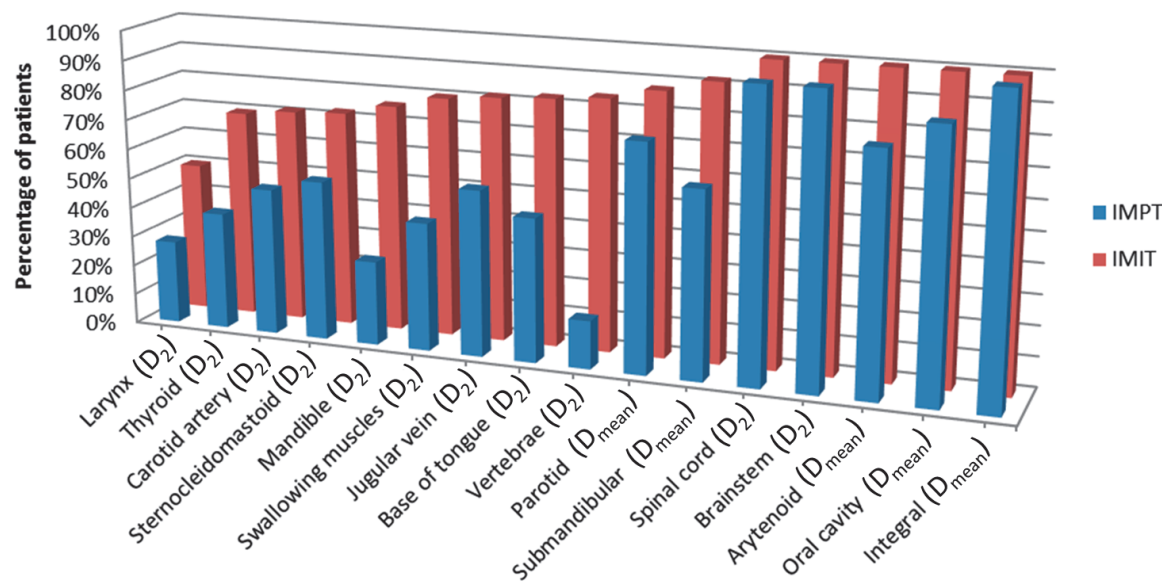

Figure 2.3 Percentage of patients receiving lower dose to the organs at risk when applying IMPT and/or IMIT compared to VMAT. The maximum dose received by $2 \%$ of that specific structure $\left(D_{2}\right)$ and the mean dose $\left(D_{\text {mean }}\right)$ both in $G y(E)$ are given.

\section{Discussion}

In this ROCOCO in silico clinical trial, through a comparison of state-of-the-art treatment plans prepared according to a strict clinical protocol, we have demonstrated, that re-RT of HNSCC patients using PT (IMPT and IMIT) can result in improved sparing of OAR when compared to photon therapy (VMAT). In most of the cases, this benefit was greater for the IMIT than for IMPT, which could be due to different beam characteristics such as sharper lateral penumbra or different spot size. While the clinical outcome data from randomized trials comparing proton versus carbon ion 
treatment are not yet available, several treatment planning studies are in line with our findings suggesting advantages of carbon ions in terms of conformity, i.e., dose to the normal tissue ${ }^{41,42}$.

However, there are confounding factors that may have influenced our findings. In this ROCOCO study, the treatment plans of all the modalities were prepared independently complying with the internal protocols applied in the individual institute's routine clinical practice. Therefore, beyond the contouring and planning goal specifications, the treatment planning procedures themselves were not necessarily alike. As a result, differences may affect the numerical outcome reported here. For instance, the different PTV margins used by the three centres for the different treatment modalities may have caused an underestimation of the value of IMPT for re-irradiating HNSCC for the numerical comparison was unfavourable. These different PTV margins may have arisen from different choices regarding the range uncertainties (see Material and Methods). Even though beyond the scope of this publication a subsequent study with predefined strict margin may shed additional light on this matter.

Also the influence of different beam angles or optimization criteria cannot be excluded. For carbon ion plans, full beam direction flexibility afforded by the gantry was exploited in the beam setups when deemed advantageous. This decision was taken at the design stage of this in silico trial and had two main reasons. In clinical routine, each of the 10 carbon ion centres currently in operation has some, often (almost) unique, limitations that start to be overcome with additional degrees of freedom by couch roll and/or pitch (www.ptcog.ch). Moreover, there are on-going efforts aiming at making gantries more available. However, research to assess the actual benefit of gantry versus fixed nozzle is warranted.

Should we all change our strategy to carbon ions then in the setting of recurrent HNSCC eligible for radical re-irradiation? We know that there have been many years of experience in using proton beams in daily clinical practice ${ }^{41}$. However, for carbon ion beams there is only little experience, with only three papers describing clinical data on the treatment of HNSCC either as single modality or combined with photons/ protons $^{44-46}$.

In this study, IMPT and IMIT plans using multi-field optimization and active scanning delivery were investigated owing to their superior delivered dose conformity. Using passive-scattering proton therapy (PSPT) would have altered the results, e.g., Kase et al. ${ }^{47}$ compared PSPT with IMPT in different primary tumour sites including nasal cavity and demonstrated that IMPT resulted in lower doses to OARs. In general, multi-field 
optimization offers superior OAR sparing compared with PSPT or pencil beam plans optimized with uniform dose per field, and this benefit can be essential in the context of re-treatment ${ }^{26,33}$.

Due to the sensitivity of pencil beam scanning proton plans to setup and range uncertainties as well as the degenerate optimal spot map solutions influenced by optimization algorithms which result in varying degrees of plan robustness, it is critical to examine robustness of PT plans ${ }^{48}$. On the other hand, optimization algorithms that explicitly incorporate robustness and can reduce uncertainties in IMPT and IMIT plans are likely to lead to less modulated fields, hence resembling SFO approach ${ }^{49}$. The influence of changing anatomy due to tumour regression and weight loss plays a more significant role in PT than in photon therapy which may necessitate the use of more frequent imaging or even adaptive planning strategies ${ }^{50-54}$. Furthermore, the presence of metal or/and associated artefacts within the treatment beam poses a problem in PT and is argued to be a patient exclusion criterion. Therefore, in our study, these artefacts were delineated and overridden to teeth and water densities, respectively, simulating their absence. This approach carries intrinsic uncertainties, which were assumed to be negligible with respect to influencing key dosimetric parameters. Richard et al. ${ }^{55}$ found that in 110 oral cavity/oropharynx radiation treatment plans artefacts were identified in $74 \%$ obscuring the CTV in $95 \%$ of these cases. In a constructed head and neck phantom, they measured PTV baseline dose ranges of $98 \%-106 \%$ and in presence of metal amalgam $66 \%-111 \%$. A potential solution may be exchanging metal fillings by composite before proton treatment planning to improve tumour visualization and dosimetry ${ }^{55}$.

An individualised radiation oncology strategy based on multifactorial decision support systems would be of great help and is currently being developed for different tumour types ${ }^{56-60}$. In this ROCOCO trial, PT is beneficial for sparing OARs in all HNSCC patients who are candidates for re-RT. However, as long as particle therapy availability is limited and comes at significantly higher costs, finding the patient who is benefitting most is highly relevant to the treating physicians as well as health insurance companies ${ }^{61}$. Therefore, in the prospective Dutch model-based approach, a plan comparison is compulsory for all non-standard indications showing that a reduction in late toxicity computed from a dose difference will actually result in clinically significant lower normal tissue complication probability (NTCP) ${ }^{63}$. This potential benefit, however, must be proven in clinical studies since toxicity is of course dependent on type of tissue, previous dose, interval and co-morbidity (e.g., vascular and metabolic comorbidity). In order to estimate this, a validated NTCP model for re-RT is needed. Developing such a re-RT-NTCP model will be difficult for it has to take into account various additional 
parameters: e.g., interval between treatments, repair capacity of the tissue as a function of dose given at first treatment and other treatments between radiations, concomitant systemic therapy (not taken into account in the current study).

In summary, the results of this in silico trial have demonstrated that PT can significantly reduce the dose to OARs whilst maintaining the prescription dose (assuming the planned dose is representative of the delivered dose); nevertheless, the exact magnitude of the clinical benefit is uncertain as a decrease in dose does not always translate into a clinically relevant decrease of toxicity risk. Proper development and validation of NTCP models for particle therapy is by no means trivial as there are many uncertainties to contend with, e.g., treatment planning, treatment delivery, relative biological effects, tumour shrinkage, and patient co-morbidities. Rapid-learning approaches along with prospective cohort studies as well as (in silico) randomised controlled trials provide possible solutions in this regard ${ }^{62}$. 


\section{References}

1. Pulte $\mathrm{D}$, Brenner $\mathrm{H}$. Changes in survival in head and neck cancers in the late 20th and early 21st century: a period analysis. Oncologist 2010;15:994-1001.

2. Ferlay J, Soerjomataram I, Ervik M, Dikshit, R et al. (Eds.), IARC Cancer Base No. 10, International Agency for Research on Cancer, Lyon, France 2013.

3. Laramore GE, Scott CB, Al-Sarraf $\mathrm{M}$ et al. Adjuvant chemotherapy for resectable squamous cell carcinomas of the head and neck: report on Intergroup Study 0034. Int J Radiat Oncol Biol Phys 1992;23:706-13.

4. Forastiere AA, Goepfert H, Maor $M$ et al. Concurrent chemotherapy and radiotherapy for organ preservation in advanced laryngeal cancer. N Engl J Med 2003;349:2091-8.

5. Cooper JS, Pajak TF, Forastiere AA et al. Postoperative concurrent radiotherapy and chemotherapy for high-risk squamous-cell carcinoma of the head and neck. N Engl J Med2004;350:1937-44.

6. Bernier J, Domenge C, Ozsahin M et al. Postoperative irradiation with or without concomitant chemotherapy for locally advanced head and neck cancer. N Engl J Med 2004;350:19-52.

7. Coatesworth AP, Tsikoudas A, MacLennan K. The cause of death in patients with head and neck squamous cell carcinoma. J Laryngol Otol 2002;116:269-71.

8. Kasperts, Slotman B., Leemans CR, Langendijk JA. A review on re-irradiation for recurrent and second primary head and neck cancer. Oral Oncol 2005;41:225-43.

9. Janot F, de Raucourt D, Benhamou E et al. Randomized trial of postoperative reirradiation combined with chemotherapy after salvage surgery compared with salvage surgery alone in head and neck carcinoma. J ClinOncol 2008;26:5518-23.

10. Lee N, Chan K, Bekelman JE et al. Salvage re-irradiation for recurrent head and neck cancer. Int J Radiat Oncol Biol Phys 2007;68:731-40.

11. Hoebers F, Heemsbergen W, Moor S et al.Reirradiation for head-and-neck cancer: delicate balance between effectiveness and toxicity.Int J Radiat Oncol Biol Phys $2011 ; 81:$ e111-8.

12. Langer CJ, Harris J, Horwitz EM et al. Phase II study of low-dose paclitaxel and cisplatin in combination with split-course concomitant twice-daily reirradiation in recurrent squamous cell carcinoma of the head and neck: Results of Radiation Therapy Oncology Group Protocol 9911. J Clin Oncol 2007;25: 4800-5.

13. Kramer NM, Horwitz EM, Cheng J et al. Toxicity and outcome analysis of patients with recurrent head and neck cancer treated with hyperfractionated split-course reirradiation and concurrent cisplatin and paclitaxel chemotherapy from two prospective phase I and II studies. Head Neck 2005;27:406-14.

14. de Crevoisier R, Bourhis J, Domenge $C$ et al. Full-dose reirradiation for unresectable head and neck carcinoma: Experience at the Gustave-Roussy institute in a series of 169 patients. J Clin Oncol 1998;16:3556-62.

15. Lee N, Chan K, Bekelman JE et al. Salvage re-irradiation for recurrent head and neck cancer. Int J Radiat Oncol Biol Phys 2007;68:731-40.

16. Wong S, Machtay M, Li Y. Locally recurrent, previously irradiated head and neck cancer: Concurrent reirradiation and chemotherapy, or chemotherapy alone? J Clin Oncol 2006;24:2653-58.

17. Salama JK, Vokesa EE. Concurrent Chemotherapy and Re-irradiation for Locoregionally Recurrent Head and Neck Cancer. Semin Oncol 2008;35:251-61.

18. Chakraborty S, Patil VM, Babu S, Muttath G, Thiagarajan SK. Locoregional recurrences after postoperative volumetric modulated arc radiotherapy (VMAT) in oral cavity cancers in a resource constrained setting: experience and lessons learned.Br J Radiol 2015 ;88:20140795.

19. Orlandi E, Giandini T, lannacone E et al. Radiotherapy for unresectable sinonasal cancers: dosimetric comparison of intensity modulated radiation terapy with coplanar and non-coplanar volumetric modulated arc therapy. Radiother Oncol 2014;113:260-6.

20. Vanetti E, Clivio A, Nicolini $G$ et al. Volumetric modulated arc radiotherapy for carcinomas of the oropharynx, hypo-pharynx and larynx: a treatment planning comparison with fixed field IMRT. Radiother Oncol 2009;92:111-7. 
21. Eekers DBP, Erik Roelofs E, Jelen $U$ et al. Data from: Benefit of particle therapy in re-irradiation of head and neck patients. Results of a multicenter in silico ROCOCO trial. CancerData. 2016. doi: doi:10.17195/candat.2016.04.2.

22. Grégoire V, Coche E, Cosnard G et al.Selection and delineation of lymph node target volumes in head and neck conformal radiotherapy. Proposal for standardizing terminology and procedure based on the surgical experience. Radiother Oncol $2000 ; 56: 135-50$.

23. Grégoire V, Eisbruch A, Hamoir M, Levendag P. Proposal for the delineation of the nodal CTV in the node-positive and the post-operative neck. Radiother Oncol 2006 ;79:15-20.

24. Grégoire $\mathrm{V}$, Ang $\mathrm{K}$, Budach $\mathrm{W}$ et al. Delineation of the neck node levels for head and neck tumors: a 2013 update. DAHANCA, EORTC, HKNPCSG, NCIC CTG, NCRI, RTOG, TROG consensus guidelines. Radiother Oncol 2014;110:172-81.

25. Lin L, Ainsley CG, Solberg TD, McDonough JE. Experimental characterization of two-dimensional spot profiles for two proton pencil beam scanning nozzles. Phys Med Biol 2014;59:493-504.

26. Quan EM, Liu W, Wu R et al. Preliminary evaluation of multifield and single-field optimization for the treatment planning of spot-scanning proton therapy of head and neck cancer. Med Phys 2013;40: 081709.

27. Both S, Shen J, Kirk M et al. Development and clinical implementation of a universal bolus to maintain spot size during delivery of base of skull pencil beam scanning proton therapy. Int J Radiat Oncol Biol Phys 2014;90:79-84.

28. DeLuca PM, Wambersie A, Seltzer SM et al. ICRU REPORT 78. Prescribing, Recording, and Reporting Proton-Beam Therapy Journal of the ICRU. ISBN 9780199543489. Published by Oxford University Press. 2007; Vol7; no2.

29. Moyers MF, Miller DW, Bush DA, Slater JD. Methodologies and tools for proton beam design for lung tumors. Int J Radiat Oncol Biol Phys 2001;49:1429-38.

30. Paganetti H. Range uncertainties in proton therapy and the role of Monte Carlo simulations. Phys Med Biol 2012;57:R99-R117.

31. Lomax AJ.Intensity modulated proton therapy and its sensitivity to treatment uncertainties 2: the potential effects of inter-fraction and inter-field motions.Phys Med Biol. 2008 Feb 21;53(4):1043-56.

32. Moyers MF1, Miller DW, Bush DA, Slater JD.Methodologies and tools for proton beam design for lung tumors. Int J Radiat Oncol Biol Phys. 2001 Apr 1;49(5):1429-38.

33. Scholz M, Kraft G. Track structure and the calculation of biological effects of heavy charged particles. Adv. Space Res1996;18:5-14.

34. Elsässer T, Krämer M, Scholz M. Accuracy of the local effect model for the prediction of biologic effects of carbon ion beams in vitro and in vivo. In. J RadiatOncol Biol Phys 2008;71:866-72.

35. Ammazzalorso F, Jelen U, Engenhart-Cabillic R, Schlegel W. Dosimetric robustness against setup errors in charged particle radiotherapy of skull base tumors. Radiat Oncol 2014;9:279.

36. Stewart FA, van der Kogel AJ. Retreatment Tolerance of Normal Tissues. Semin Radiat Oncol 1994;4: 103-11.

37. Stewart FA. Re-treatment after full-course radiotherapy: Is it a viable option? Acta Oncol 1999;38: 855-62.

38. Nieder C, Milas L, Ang KK. Tissue tolerance to reirradiation. Semin Radiat Oncol 2000;10:200-9.

39. ICRU. International Commission on Radiation Units and Measurements. Prescribing, recording, and reporting photon-beam intensity-modulated radiation therapy (IMRT). ICRU Report 83. J ICRU, 10 (2010), pp. 1-106.

40. Roelofs E, Persoon L, Qamhiyeh S et al. Design of and technical challenges involved in a framework for multicentric radiotherapy treatment planning studies. Radiother Oncol $2010 ; 97: 567-71$.

41. Weber U, Kraft G. Comparison of carbon ions versus protons. Cancer J 2009;15:325-32.

42. Grün R, Friedrich $T$, Krämer $M$ et al. Assessment of potential advantages of relevant ions for particle therapy: a model based study. Med Phys 2015;42:1037-47.

43. Takagi $\mathrm{M}$, Demizu $\mathrm{Y}$, Hashimoto $\mathrm{N}$ et al. Treatment outcomes of particle radiotherapy using protons or carbon ions as a single-modality therapy for adenoid cystic carcinoma of the head and neck. Radiother Oncol $2014 ; 113: 364-70$. 
44. Koto $M$, Hasegawa $A$, Takagi $R$ et al. Carbon ion radiotherapy for locally advanced squamous cell carcinoma of the external auditory canal and middle ear. Head Neck 2016;38(4):512-6.

45. Morimoto $\mathrm{K}$, Demizu $\mathrm{Y}$, Hashimoto $\mathrm{N}$ et al. Particle radiotherapy using protons or carbon ions for unresectable locally advanced head and neck cancers with skull base invasion. Jpn J Clin Oncol 2014;44:428-34.

46. Jensen AD, Poulakis M, Nikoghosyan AV at al. High-LET radiotherapy for adenoid cystic carcinoma of the head and neck: 15 years' experience with raster-scanned carbon ion therapy. Radiother Oncol 2015;S0167-8140:00245-5.

47. Kase $\mathrm{Y}$, Yamashita $\mathrm{H}$, Fuji $\mathrm{H}$ et al. A treatment planning comparison of passive-scattering and intensitymodulated Proton therapy for typical tumor sites. J Radiat Res 2012;53:272-80.

48. Albertini F, Hug EB, Lomax AJ. The influence of the optimization starting conditions on the robustness of intensity-modulated proton therapy plans. Phys Med Biol 2010;55:2863-78.

49. Liu W, Frank SJ, Li X et al. Effectiveness of Robust Optimization in Intensity-Modulated Proton Therapy Planning for Head and Neck Cancers. Med Phys 2013;40:051711.

50. Hermans BC, Persoon LC, Podesta M, Hoebers FJ, Verhaegen F, Troost EG. Weekly kilovoltage conebeam computed tomography for detection of dose discrepancies during (chemo)radiotherapy for head and neck cancer. Acta Oncol 2015;54:1483-9.

51. Steiner E, Stock M, Kostresevic B et al. Imaging dose assessment for IGRT in particle beam therapy. Radiother Oncol 2013;109:409-13.

52. Kraan AC, van de Water S., Teguh DN et al. Dose uncertainties in IMPT for oropharyngeal cancer in the presence of anatomical, range, and setup errors. Int J Radiat Oncol Biol Phys 2013;87 888-96.

53. Müller BS, Duma MN, Kampfer S et al.Impact of interfractional changes in head and neck cancer patients on the delivered dose in intensity modulated radiotherapy with protons and photons. Phys Med 2015;31:266-72.

54. Jakobi A, Bandurska-Luque A, Stützer K et al. Identification of Patient Benefit From Proton Therapy for Advanced Head and Neck Cancer Patients Based on Individual and Subgroup Normal Tissue Complication Probability Analysis. Int J Radiat Oncol Biol Phys. 2015 Aug 1;92(5):1165-74.

55. Richard P, Sandison G, Dang Q, Johnson B, Wong T, Parvathaneni U. Dental amalgam artefact: Adverse impact on tumor visualization and proton beam treatment planning in oral and oropharyngeal cancers. Pract Radiat Oncol 2015;5:e583-8.

56. Ramaekers BL, Grutters JP, Pijls-Johannesma M, Lambin P, Joore MA, Langendijk JA. Protons in headand-neck cancer: bridging the gap of evidence. Int J Radiat Oncol Biol Phys 2013;85:1282-8.

57. Lambin P, Petit SF, Aerts HJ, van Elmpt WJ et al. The ESTRO Breur Lecture 2009. From population to voxel-based radiotherapy: exploiting intra-tumour and intra-organ heterogeneity for advanced treatment of non-small cell lung cancer. Radiother Oncol 2010;96:145-52.

58. Lambin P, van Stiphout RG, Starmans $\mathrm{MH}$ et al. A. Predicting outcomes in radiation oncology-multifactorial decision support systems. Nat Rev Clin Oncol 2013;10:27-40.

59. Lambin P, Roelofs E, Reymen B et al. A. Rapid Learning health care in oncology' - An approach towards decision support systems enabling customised radiotherapy'. Radiother Oncol 2013;109:159-64.

60. Lambin P, Zindler J, Vanneste B et al. Modern Clinical Research: How Rapid learning health care and cohort multiple randomised clinical trials complement traditional evidence based medicine. Acta Oncol 2015;54:1289-300.

61. Langendijk JA, Lambin P, De Ruysscher D, Widder J, Bos M, Verheij M. Selection of patients for radiotherapy with protons aiming at reduction of side effects: the model-based approach. Radiother Oncol 2013;107:267-73.

62. Widder J, Schaaf van der A, Lambin P et al. The quest for evidence for proton therapy: the model-based approach and precision medicine. Int J Radiat Oncol Biol Phys. 2016;95(1):30-6.

63. Cheng $Q$, Roelofs E, Ramaekers B et al. EP-1480: Development and validation of a proton decision support system comparing dose, toxicity and cost-effectiveness. RadiotherOncol. 2015;115:S804. 


\section{Supplemental materials}

\section{a. $D_{2}$ Brainstem cumulative}

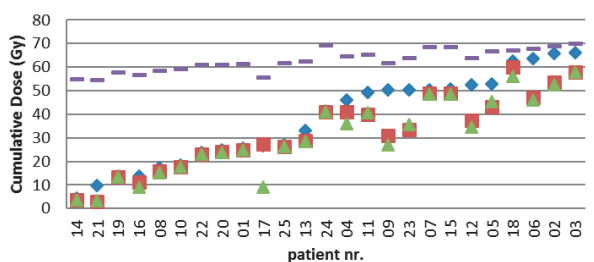

c. $D_{2}$ Spinal Cord cumulative

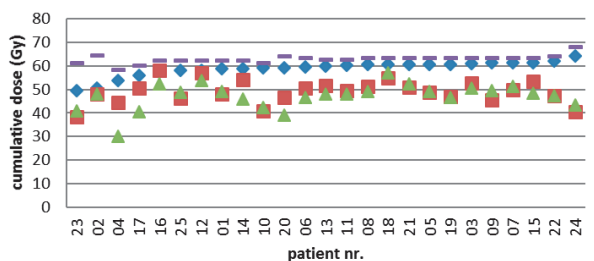

b. $D_{2}$ Mandible cumulative

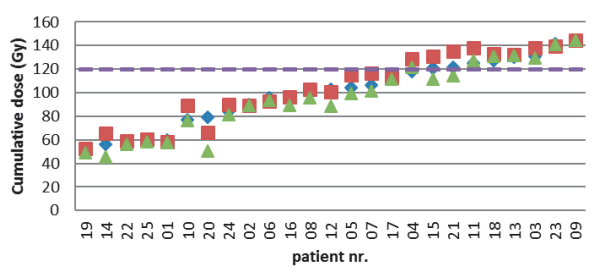

d. $D_{2}$ Larynx cumulative

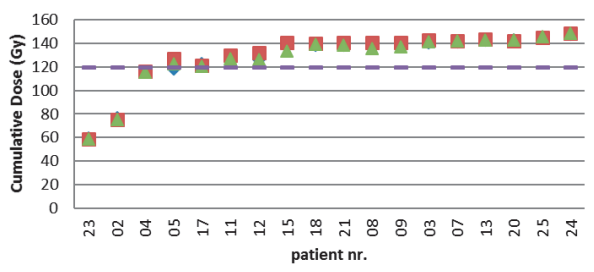

Figure S2.1 Dosimetric metrices cumulative for selected OARs per patient for VMAT, IMPT and IMIT treatment plans.

The dose (Gy) is depicted per patient for VMAT (diamond), IMPT (square) and IMIT (triangle) and is sorted for VMAT. The individually calculated dose constraint per patient is taking the first treatment into account (line): a) the maximum dose to $2 \%$ of the brainstem $\left.\left(D_{2}\right), b\right)$ the $D_{2}$ of the mandible, c) the $D_{2}$ of the spinal cord, d) the $D_{2}$ of the mandible, all in in Gy(equivalent). 


\section{a. Integral dose ( $\left.D_{\text {mean }}\right)$}

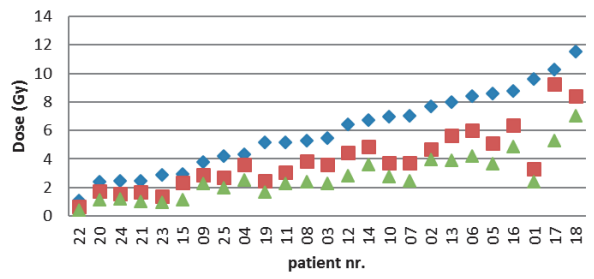

c. $D_{2}$ Spinal cord

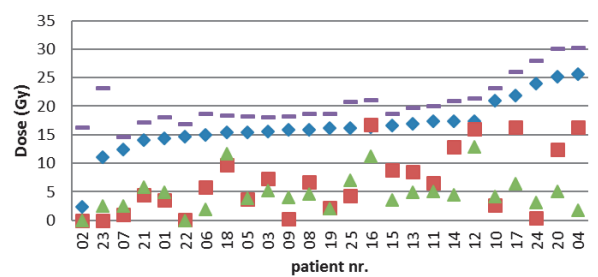

b. $D_{2}$ Larynx

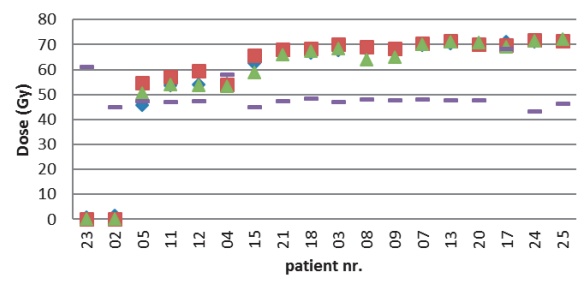

d. $D_{2}$ Mandible

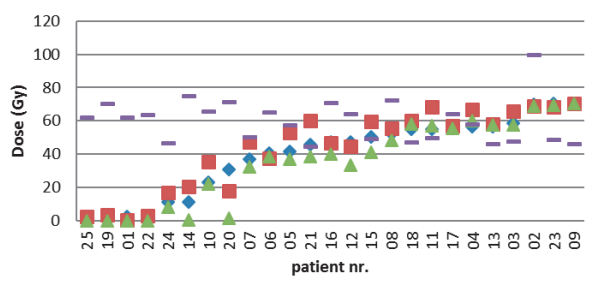

- VMAT IIMPT $\triangle$ IMIT - CONSTRAINT

Figure S2.2 Dosimetric metrices for selected OARs per patient for VMAT, IMPT and IMIT treatment plans. The dose (Gy) is depicted per patient for VMAT (diamond), IMPT (square) and IMIT (triangle) is sorted for VMAT. The individually calculated dose constraint per patient is taking the first treatment into account (line): a) the integral Body dose ( $D_{\text {mean }}$ ) minus the CTV, b) the maximum dose to $2 \%$ of the of the larynx $\left.\left(D_{2}\right), c\right)$ the maximum dose to $2 \%$ of the spinal cord $\left(D_{2}\right)$ and d) the maximum dose to $2 \%$ of the mandible $\left(D_{2}\right)$, all in $G y(E)$. 


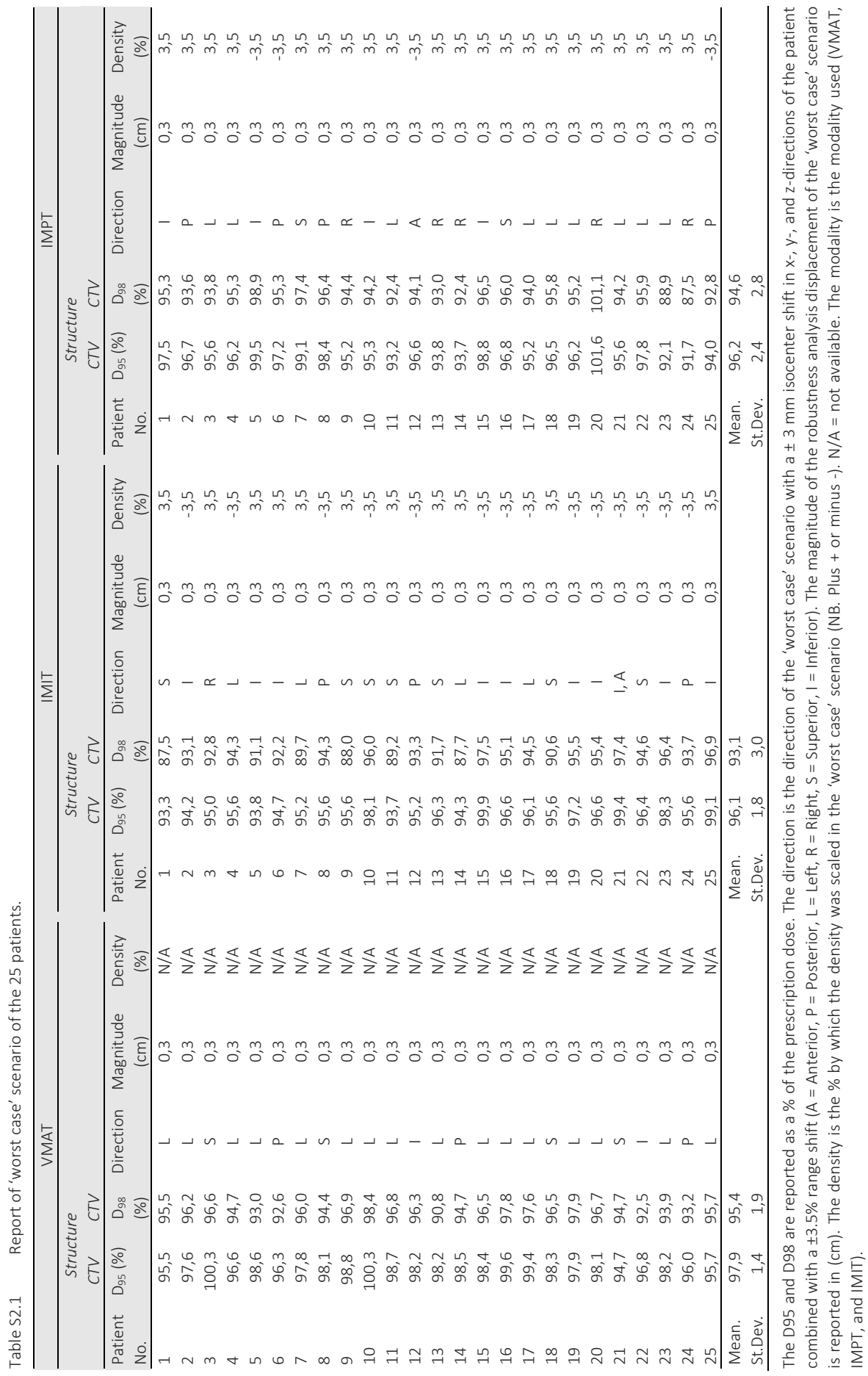





\section{Chapter

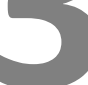

Intensity-modulated proton therapy decreases dose

to organs at risk in low-grade glioma patients: results of a multicentric in silico ROCOCO trial

Daniëlle B.P. Eekers , Erik Roelofs, Macarena Cubillos-Mesias, Charles Niël, Robert J. Smeenk, Ann Hoeben, Andre Minken, Marlies Granzier-Peeters, Geert O.R.J Janssens, Johannes H.A.M. Kaanders, Philippe Lambin, Esther G.C. Troost Acta Oncologica, 2018. In press 


\section{Abstract}

\section{Background and purpose}

Patients with low-grade glioma (LGG) have a prolonged survival expectancy due to better discriminative tumor classification and multimodal treatment. Consequently, long-term treatment toxicity gains importance. Contemporary radiotherapy techniques such as intensity-modulated radiotherapy (IMRT), volumetric modulated arc therapy (VMAT), Tomotherapy (TOMO) and intensity-modulated proton therapy (IMPT) enable high-dose irradiation of the target but they differ regarding delivered dose to organs at risk (OARs). The aim of this comparative in silico study was to determine these dosimetric differences in delivered doses.

\section{Material and methods}

Imaging datasets of twenty-five LGG patients having undergone postoperative radiotherapy were included. For each of these patients, in silico treatment plans to a total dose of $50.4 \mathrm{~Gy}$ to the target volume were generated for the four treatment modalities investigated (i.e., IMRT, VMAT, TOMO, IMPT). Resulting treatment plans were analyzed regarding dose to target and surrounding OARs comparing IMRT, TOMO and IMPT to VMAT.

\section{Results}

In total 100 treatment plans (4 per patient) were analyzed. Compared to VMAT the IMPT mean dose ( $\left.D_{\text {mean }}\right)$ for 9 out of 10 (90\%) OARs was statistically significantly $(p<0.02)$ reduced, for TOMO this was true in $3 / 10(30 \%)$ patients and for $1 / 10(10 \%)$ patients for IMRT. IMPT was the prime modality reducing dose to the OARs followed by TOMO.

\section{Discussion}

The low dose volume to the majority of OARs was significantly reduced when using IMPT compared to VMAT. Whether this will lead to a significant reduction in neurocognitive decline and improved quality of life is to be determined in carefully designed future clinical trials. 


\section{Introduction}

Gliomas are the most common tumors of the central nervous system (CNS), with an annual incidence of 5.4 per 100.000 in Europe ${ }^{1}$. Low-grade gliomas (LGG), classified as World Health Organization (WHO) grade 2, typically occur in younger adults $\left(2^{\text {nd }}-5^{\text {th }}\right.$ decade) and are diffusely infiltrative tumors. Most LGG show a protracted disease course; however, significant differences in survival rates have been identified. Consecutively, molecular parameters, such as 1p19q co-deletions, IDH mutation, TERT promotor mutations, and EGFR-amplifications and -mutations have found their way into the novel WHO classification of CNS tumors, revised in 2016 $2-4$. This integrated diagnosis of gliomas is now leading the neuro-oncology field towards multimodal treatment schedules capable of increasing overall survival of diffuse gliomas with an aggressive clinical behavior ${ }^{5,6}$. Multimodal treatment schedules include neurosurgical resection if feasible and adjuvant treatment with radiation therapy as well as chemotherapy ${ }^{6}$. With increasing survival of LGG patients, therapy induced toxicity, such as neurocognitive decline caused by radiotherapy, which potentially decreases the quality of life of these patients, should be minimized $^{7}$

There has been an impressive progress in recent photon-based radiotherapy techniques, moving from 3D conformal radiotherapy (3D-CRT) to intensity-modulated radiotherapy (IMRT), volumetric modulated arc therapy (VMAT), and tomotherapy (TOMO), delivering high radiation dose to the target and low doses to surrounding organs at risk (OARs) ${ }^{8}$. Examples of this progress are the RTOG 0933 phase 2 clinical study on conformal hippocampus sparing whole brain radiotherapy showing a preservation of memory and quality of life, and the ongoing trial on hippocampussparing prophylactic cranial irradiation using VMAT, IMRT or TOMO (NCT01780675) ${ }^{9}$.

As a result of their physical characteristics, protons deposit a low entry dose followed by maximum dose delivery the Bragg peak, and zero dose after the dose fall-off at the distal edge of the Bragg peak, resulting in a superior sparing surrounding OARs compared to photons. Conversely, the tumor control probability may be increased at maintained OAR dose or by exploiting the somewhat higher relative biological effectiveness ${ }^{10-13}$. Consequently, physicians, patients, and health insurance companies are interested in the role of proton therapy (PT), e.g., regarding its possible reduction of toxicity in LGG and head-and-neck cancer patients, amongst others.

To assess the potential dosimetric gains of intensity modulated proton therapy (IMPT) for individual LGG patients, we conducted an international multicenter in silico treatment planning study within the Radiation Oncology Collaborative Comparison (ROCOCO) group in a cohort of 25 LGG patients retrospectively retrieved from two Dutch radiotherapy departments ${ }^{14-16}$. 


\section{Materials and methods}

\section{Study population}

We retrospectively retrieved radiation treatment plans and underlying imaging data, i.e., computed tomography (CT) and magnetic resonance imaging (MRI), of $25 \mathrm{WHO}$ grade 2 LGG patients who had undergone radiation treatment (VMAT or IMRT) at the Radboud University Medical Centre (Radboudumc), Nijmegen (The Netherlands), or at the Department of Radiation Oncology of Maastricht University Medical Center (MAASTRO clinic), Maastricht (The Netherlands). These patients had previously undergone a gross total resection or biopsy depending on the localization of the tumor. No prior systemic therapy had been administered. This ROCOCO in silico trial was approved by the MAASTRO clinic institutional review board and was registered on clinicaltrial.gov ID: NCT NCT02607397. Data are available on www.cancerdata.org ${ }^{17}$.

\section{Target volume and OAR definition}

An individual head support and thermoplastic mask was used in all patients. The gross tumor volume (GTV) was delineated as the resection cavity, encompassing any residual/recurrent macroscopic tumor on the planning-CT (2 or $3 \mathrm{~mm}$ slices) fused with the (pre- and post-surgical) MRI (T1-weighted with contrast agent and T2weighted/FLAIR images) using image registration of Eclipse ${ }^{\mathrm{TM}}$ (V11.0 Varian Medical Systems, Palo Alto, CA). The clinical target volume (CTV) was defined by the treating radiation oncologist as the GTV with a $1 \mathrm{~cm}$ margin, corrected for anatomical boundaries, according the local guidelines at Radboudumc and MAASTRO clinic. For this in silico study, the CTV was distributed to the participating centers. Taking into account different photon techniques and the individual institution's margin recipes, the CTV was expanded to the planning target volume (PTV): linear accelerator (LINAC) based VMAT and IMRT utilized $2 \mathrm{~mm}$ accounting for setup errors, and helical tomotherapy based IMRT (TOMO) used a $3 \mathrm{~mm}$ margin based on the individual institution's clinically derived margins. Intensity-modulated proton therapy (IMPT) used a setup uncertainty of $2 \mathrm{~mm}$ and a range uncertainty of 3.5\%. For all 25 patients included in this in silico study, the OARs were outlined by one dosimetrist (M.G.) and supervised by a radiation oncologist (D.E.) according the atlas by Eekers et al. ${ }^{18}$ (see Table 3.1 for the list of OARs). A double-sided OAR was termed 'contralateral' when located at the contralateral hemisphere and not included in the CTV. Whenever the tumor was centrally located or both double-sided OARs were located within the CTV, this OAR was named 'bilateral'. Dental fillings and associated artifacts were delineated and the density was overridden to that of teeth or soft tissue, respectively. Dental fillings within 
the treatment beam were an exclusion criterion for IMPT. There was no correction for the use of intravenous contrast during the planning-CT.

\section{Dose prescription}

For simplicity reasons, all doses are reported in Gy equivalents (GyE), taking into account the relative biological effectiveness (RBE) of 1 for photons and 1.1 for protons. The prescribed dose to the target was $50.4 \mathrm{~Gy}$ in 28 fractions of $1.8 \mathrm{~Gy}$, such that at least $99 \%$ of the volume had to be covered by $95 \%$ of the dose $\left(\mathrm{V}_{95 \%}=99 \%\right)$. All proton (IMPT) and photon (VMAT, IMRT, TOMO) treatment plans were generated in centers with significant clinical experience in treatment planning.

\section{Dose constraints}

The dose limits and priorities for the OARs were defined in the protocol of this in silico comparative study (see Table 3.1). The dose limits to the brainstem, brain, spinal cord, chiasm, optic nerves and retina were not to be exceeded. Attempts were made to minimize the dose to the other OARs without causing underdosage of the PTV F0.4Gy.

Table 3.1 Tolerance dose and planning priority per organ at risk in Gy(RBE).

\begin{tabular}{lllc}
\hline OAR & Tolerance & Priority \\
\hline Brain Stem & $\mathrm{D}_{2 \%} 54 \mathrm{~Gy}$ & 1 \\
Brain & $\mathrm{D}_{2 \%} 60 \mathrm{~Gy}$ & 1 \\
Spinal cord & $\mathrm{D}_{2 \%} 50 \mathrm{~Gy}$ & 1 \\
Chiasm \& optic nerve & $\mathrm{D}_{0.1 \mathrm{cc}} 55 \mathrm{~Gy}$ & 1 \\
Retina & $\mathrm{D}_{0.1 \mathrm{cc}} 45 \mathrm{~Gy}$ & 1 \\
Cornea & $\mathrm{D}_{0.1 \mathrm{cc}} 30 \mathrm{~Gy}$ & 2 \\
Lacrimal gland & $\mathrm{D}_{\text {mean }} 30 \mathrm{~Gy}$ & 2 \\
Cochlea & $\mathrm{D}_{\text {mean }} 45 \mathrm{~Gy}$ & 3 \\
Lens & $\mathrm{D}_{0.1 \mathrm{cc}} 30 \mathrm{~Gy}$ & 4 \\
Hippocampus & $\mathrm{D}_{\text {mean }} 9 \mathrm{~Gy}$ & 4 \\
Pituitary gland & $\mathrm{D}_{\text {mean }} 45 \mathrm{~Gy}$ & 4 \\
Posterior cerebellum & $\mathrm{D}_{\text {mean }}$ ALARA & 4 \\
\hline
\end{tabular}

Dose limiting, exceeding this dose is not permitted

Only dose limiting if the OAR was not part of the CTV

Not dose limiting if the contralateral organ is preserved

Goal limits, not dose limiting

OAR=organ at risk, $D_{2} \%$ is the maximum dose to $2 \%$ of the volume of the OAR, $D_{\text {mean }}=$ mean dose, $D_{0.1 c c}$ is the maximum dose reported in case $2 \%$ is smaller than $0.1 \mathrm{cc}$. ALARA = as low as reasonably achievable. 


\section{Photon planning}

The IMRT and VMAT treatment plans were created at MAASTRO clinic using Eclipse ${ }^{\mathrm{TM}}$ (v11.0 Varian Medical Systems, Palo Alto, CA). For VMAT plans, one or two $10 \mathrm{MV}$ halfor whole arcs were used. For IMRT beam angle optimization was used for the optimal beam arrangement, using 4 to 7 beams of $10 \mathrm{MV}$ each. The TOMO treatment plans were created at Radiotherapiegroep Deventer using Accuray Hi-Art Planning Station (v5.1.0.4, Accuray, Sunnyvale, CA). The photon plans using a PTV margin were considered to be innately robust relative to PT, assuming the PTV to be sufficient to produce a robust plan. A grid size of $2-3 \mathrm{~mm}$ was used for all modalities depending on the slice spacing of the dataset.

\section{Proton planning}

Proton treatment plans were calculated using RayStation (v4.65.99, RaySearch Laboratories AB, Stockholm, Sweden) using IMPT for beam delivery with pencil beam scanning (PBS). Treatment plans were created for each patient using a robust optimized plan with mostly two radiation beams. The air-gap used between the patient surface and the range shifter was $2 \mathrm{~cm}$. The beam direction was chosen individually for every patient in order to spare the OARs and healthy brain tissue, and to avoid passing through air cavities. The minimum energy of the beam model used was $100 \mathrm{MeV}$ with a spot size sigma ranging from $4 \mathrm{~mm}(220 \mathrm{MeV})$ to $8 \mathrm{~mm}(100 \mathrm{MeV})$ in the isocenter. These robust plans were generated optimizing the target coverage for the CTV, thus no PTV concept was used, including a setup uncertainty of $2 \mathrm{~mm}$ and a range uncertainty $3.5 \%$, and considering 21 scenarios for the optimization process. A range shifter of approximately $7.5 \mathrm{~cm}$ water equivalent and a calculation grid of $3 \mathrm{~mm}$ were used in all cases.

\section{Storage of imaging datasets}

The datasets were stored and exchanged through the secured collaborative MISTIR platform hosted by MAASTRO clinic. Quality assurance procedures were applied to assess the necessity of corrections of potential transformations during import and export in the respective treatment planning systems ${ }^{19}$.

\section{Data evaluation and statistical analysis}

For each treatment plan, the mean $\left(D_{\text {mean }}\right)$ and maximum dose $\left(D_{\max }\right)$ as well as the near-maximum dose, defined as the highest dose to $2 \%$ of the volume $\left(D_{2} \%\right)$ or to $0.1 \%$ $\left(D_{0.1 c c}\right)$ in case $2 \%$ was smaller than $2 \mathrm{cc}$, were calculated for each OAR and CTV ${ }^{20}$. The 
mean dose to the imaged part of the patient (body contour) minus CTV was defined as the mean integral dose (ID).

For statistical analyses, an in-house developed script in Matlab (version 2017a, The MathWorks, Natick, MA) was used to extract dose-volume-histograms (DVH) metrics from the $3 D$ dose distributions that were uploaded by the participating centers. To allow for a direct comparison between all treatment modalities, the doses to the CTV were considered as no PTV was used for protons. The doses were scaled such that $99 \%$ of the CTV received exactly $100 \%$ of the prescribed dose (50.4 Gy). Whenever needed, scaling was increased to be sure that the GTV was covered with at least $50.4 \mathrm{~Gy}$. This was required in four patients with a factor between 0.98 and 1.16 .

Considering the fact that VMAT was used for the actual treatment of the 25 LGG patients included in this study, it was considered the gold standard for the comparative analyses. The DVH metrics and doses to OARs were statistically compared using twotailed, Wilcoxon signed-rank test to determine the significance of pairwise differences compared to VMAT. Accounting for multiple testing and applying a Bonferroni correction, a p-value $<0.02$ was considered statistically significant. The Van 't Riet ${ }^{21}$ conformity number ( 0 to 1 ) was used to describe the conformity of the CTV coverage, with 1 indicating a perfect conformity.

To evaluate the TPS performance of robust IMPT planning, six random patients were evaluated with each having 26 scenarios for setup $(2 \mathrm{~mm})$ and density (3.5\%) changes. Next, the $V_{95 \%}$ of the CTV prescribed dose was calculated. To quantify the variability in the results, the Coefficients of Variation ( $C V=$ standard deviation over mean) were determined.

\section{Results}

In total 100 treatment plans for the twenty-five patients with LGG patients were calculated and analyzed. An example of the treatment plans for a patient is presented in Figure 3.1. The dose coverage of the $\mathrm{CTV}_{50.4 \mathrm{~Gy}}$ was statistically significantly better for TOMO than for VMAT $\left(p=0.02 ; V_{95 \%}=99.9 \%\right)$, overall the coverage was excellent for all modalities with the volume receiving $95 \%$ of the prescribed dose $\left(\mathrm{V}_{95 \%}\right)$ of the $\mathrm{CTV}_{50.4 G \mathrm{y}}$ ranging from 99.7 to $100 \%$ (comparison to gold standard VMAT given in Table 3.2). The conformity expressed using the Van 't Riet ${ }^{21}$ conformity number showed that VMAT plans had the highest conformity $(0.74)$, followed by TOMO $(0.72 ; p<0.02)$, IMRT (0.69; $\mathrm{p}<0.02)$, and IMPT (0.69; $\mathrm{p}<0.02$; Table 3.2). In accordance with robust photon plans, the variability in robust planning for the six evaluated IMPT cases proved to be very limited with an average CV of 0.07\% (range 0.05-0.12\%; Supplementary Table S3.1). 
The mean integral dose was significantly increased for TOMO (8.3 Gy; $\mathrm{p}<0.02$ ) and IMRT (7.9 Gy, p<0.02) and decreased for IMPT (5.6Gy, p<0.02) compared to VMAT (7.8 Gy). Table 2 shows the average scaled values of the $D_{\text {mean }}$ and $D_{2}$ for the OARs delivered by IMRT, TOMO and IMPT in comparison to VMAT; overall, the $D_{\text {mean }}$ of the OARs statistically significantly differed from VMAT for IMRT, TOMO, and IMPT, being $1 / 10$ (10\%), $3 / 10(30 \%)$, and $9 / 10(90 \%)$, respectively. For the $D_{2 \%}$, a statistically significant dose reduction was found in $3 / 12(25 \%), 6 / 12$ (50\%) and 10/12 (83\%) of the OARs, respectively. IMPT was the prime modality reducing dose to the OARs followed by TOMO. The pituitary gland was best spared by TOMO (Table 3.2).

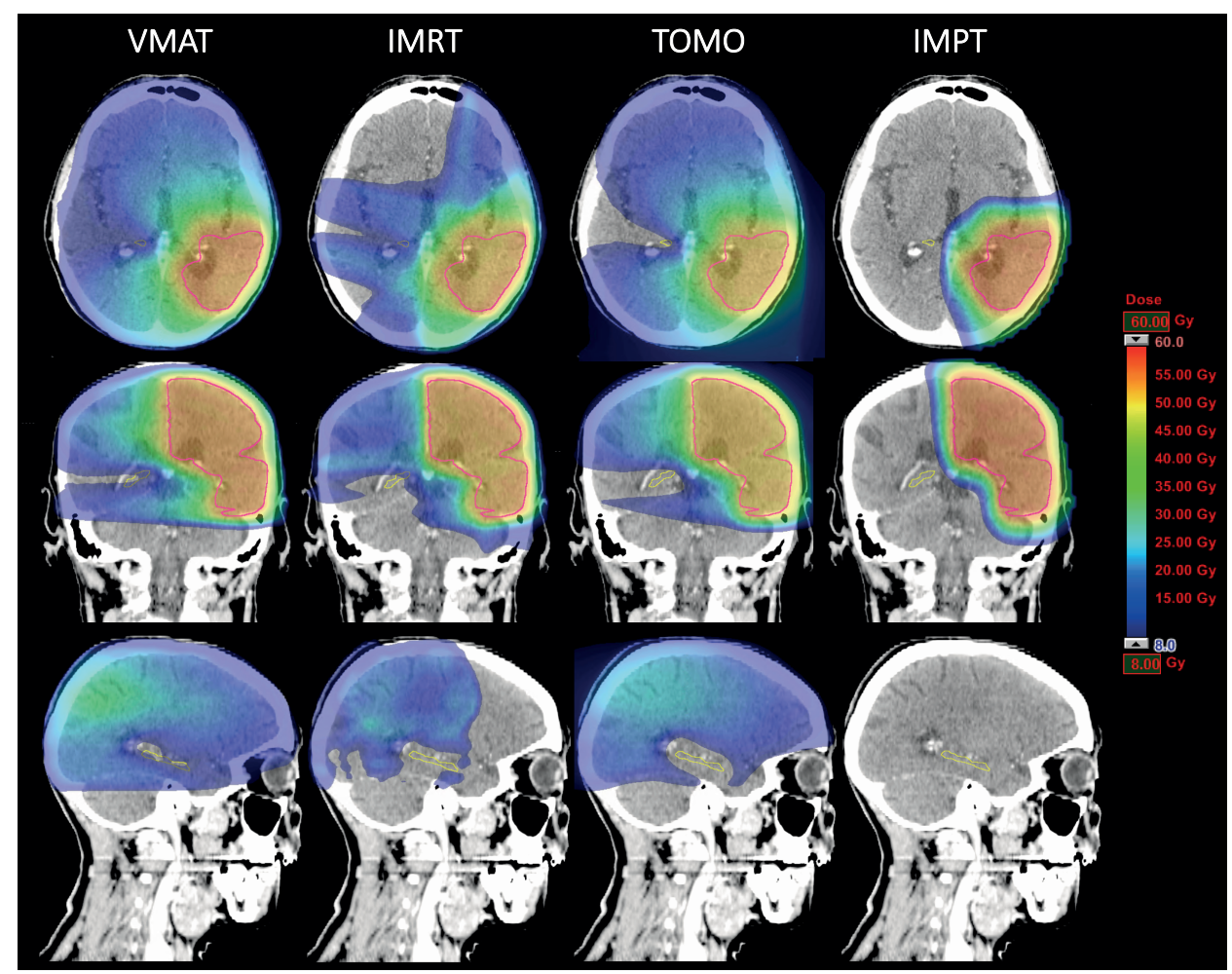

Figure 3.1 Example of a radiation treatment plans for a patient with a LGG parieto-occipitally in the left hemisphere. The CTV (pink), hippocampus (yellow) and dose distribution (ranging from low dose depicted in blue to high dose in red) are given for the VMAT (A), TOMO (B), IMRT (C) and IMPT (D) treatment plans, in the transverse (upper row), frontal (middle row) and sagittal (lower row) view. Of note is the large low-dose bath when using either of the photon techniques (A-C). 
Table 3.2 Dose and coverage parameters per organ at risk or target volume per treatment modality (significance calculated in comparison to the gold standard VMAT).

\begin{tabular}{|c|c|c|c|c|}
\hline Organ at risk & VMAT $D_{2 \%}$ & $\mathrm{IMRT} \mathrm{D}_{2 \%}$ & TOMO $D_{2 \%}$ & IMPT D $2 \%$ \\
\hline Brain & $52.7(1.1)$ & $53.4(1.7)^{*}$ & $52.7(0.47)$ & $52.4(0.46)$ \\
\hline Brainstem & $40.7(16.7)$ & $40.5(17.9)$ & $39.5(16.5)$ & $39.2(19.8)$ \\
\hline Chiasm & $37.7(17.2)$ & $36.4(18.9)$ & $33.7(19.2)^{*}$ & $32.8(22.5)^{*}$ \\
\hline Cornea ipsi \& bilateral & $13.8(5.7)$ & $14.1(9.0)$ & $12.1(5.7)^{*}$ & $5.1(7.0)^{*}$ \\
\hline Cornea contralateral & $12.5(4.8)$ & $9.6(5.4)^{*}$ & $11.0(4.7)$ & $1.3(2.4)^{*}$ \\
\hline Lens ipsi \& bilateral & $5.2(1.6)$ & $6.9(5.5)$ & $4.4(2.1)$ & $1.4(1.9)^{*}$ \\
\hline Lens contralateral & $5.2(1.5)$ & $5.1(2.9)$ & $4.2(1.8)$ & $0.52(1.3)^{*}$ \\
\hline Optic nerve ipsi \& bilateral & $31.6(17.5)$ & $30.7(18.4)$ & $27.4(18.7)^{*}$ & $27.9(22.8)$ \\
\hline Optic nerve contralateral & $26.4(14.7)$ & $21.5(16.0)^{*}$ & $16.4(12.9)^{*}$ & $16.0(19.8)^{*}$ \\
\hline Retina ipsi \& bilateral & $17.6(9.0)$ & $18.0(11.9)$ & $14.0(7.0)^{*}$ & $10.7(12.2)^{*}$ \\
\hline Retina contralateral & $14.6(5.7)$ & $10.9(6.3)^{*}$ & $10.2(4.2)^{*}$ & $1.8(3.3)^{*}$ \\
\hline Spinal cord & $3.0(10.3)$ & $3.8(11.1)$ & $3.1(10.0)^{*}$ & $2.2(10.5)^{*}$ \\
\hline Organ at risk / target volume & VMAT $D_{\text {mean }}$ & IMRT D mean & TOMO $D_{\text {mean }}$ & IMPT D mean \\
\hline Cerebellum anterior & $21.8(15.8)$ & $21.8(16.2)$ & $20.9(14.4)$ & $16.2(15.8)^{*}$ \\
\hline Cerebellum posterior & $7.1(9.8)$ & $8.5(11.1)^{*}$ & $7.4(9.4)$ & $5.6(9.2)^{*}$ \\
\hline Cochlea ipsi \& bilateral & $15.7(17.0)$ & $16.0(16.7)$ & $12.2(12.9)^{*}$ & $19.7(21.1)$ \\
\hline Cochlea contralateral & $7.4(6.5)$ & $5.8(6.3)$ & $4.0(3.1)$ & $0.015(0.036)^{*}$ \\
\hline Hippocampus ipsi \& bilateral & $32.2(17.8)$ & $33.3(17.4)$ & $31.5(18.6)$ & $32.8(19.6)$ \\
\hline Hippocampus contralateral & $10.4(7.1)$ & $10.8(8.4)$ & $7.6(6.1)$ & $2.2(5.0)^{*}$ \\
\hline Hippocampus left \& right & $23.1(10.4)$ & $24.0(10.9)$ & $21.4(10.5)$ & $19.9(10.5)^{*}$ \\
\hline Lacrimal gland ipsi \& bilateral & $12.3(6.1)$ & $10.8(7.6)$ & $8.0(4.3)^{*}$ & $3.5(4.7)^{*}$ \\
\hline Lacrimal gland contralateral & $10.3(4.3)$ & $7.3(4.1)^{*}$ & $5.8(2.3)^{*}$ & $0.17(0.47)^{*}$ \\
\hline Pituitary gland & $24.0(14.3)$ & $23.0(14.8)$ & $13.0(10.1)^{*}$ & $22.7(19.7)$ \\
\hline Integral dose to body & $7.8(3.5)$ & $7.9(3.6)$ & $8.3(3.6)^{*}$ & $5.6(2.8)^{*}$ \\
\hline $\mathrm{CTV}_{50.4 \mathrm{~Gy}}$ & $52.0(1.0)$ & $52.4(1.6)^{*}$ & $52.1(0.37)$ & $51.7(0.36)$ \\
\hline GTV & $52.2(1.0)$ & $52.6(1.6)^{*}$ & $52.6(0.43)^{*}$ & $51.6(0.35)^{*}$ \\
\hline Structure name & VMAT $V_{95 \%}$ & IMRT V V95\% & TOMO V95\% & IMPT v95\% \\
\hline $\mathrm{CTV}_{50.4 G \mathrm{y}}$ & $99.8(0.75)$ & $99.7(1.5)$ & $99.9(0.13)^{*}$ & $100.0(0.019)$ \\
\hline
\end{tabular}

$D_{2 \%}=$ Dose to $2 \%$ of the structure in Gy $(R B E) ; D_{\text {mean }}=$ mean dose to organ at risk or target volume in $G y(R B E) ;$ $\mathrm{V}_{95 \%}=$ organ at risk / target volume receiving $95 \%$ of the dose; $\mathrm{CN}=$ conformity number; ${ }^{*}=\mathrm{p}<0.02$.

The brain volume receiving a dose up to $30 \mathrm{~Gy}\left(\mathrm{~V}_{5 \mathrm{~Gy}}-\mathrm{V}_{30 \mathrm{~Gy}}\right)$ was statistically significantly reduced using IMPT compared to VMAT (comparison of the percentage of hippocampus, posterior cerebellum, and brain to the gold standard VMAT is given in Table 3.3); the $\mathrm{V}_{5 \mathrm{~Gy}}$ being $85 \mathrm{cc}$ (SD $12 \mathrm{cc}$ ) for VMAT versus $49 \mathrm{cc}$ (SD $16.3 \mathrm{cc}$ ) for IMPT and the $\mathrm{V}_{20 \mathrm{~Gy}} 56 \mathrm{cc}$ (SD $19 \mathrm{cc}$ ) versus $39 \mathrm{cc}$ (SD $15 \mathrm{cc}$ ), respectively [see Figure 3.2 for $20 \mathrm{~Gy}(\mathrm{RBE})$ volume]. Besides for brain, the low dose volumes to the hippocampus (bilateral and contralateral) and posterior cerebellum were statistically significantly reduced using IMPT whereas the high dose volumes to hippocampus bi- and ipsilateral, posterior cerebellum and brain increased using IMPT compared to VMAT (Figure 3.3). 
Table 3.3 Absolute percentage of the hippocampus, posterior cerebellum, and brain volumes receiving a dose between 5 and $50 \mathrm{~Gy}(\mathrm{RBE})$ presented per treatment modality (statistical comparison versus the gold standard being VMAT).

\begin{tabular}{|c|c|c|c|c|}
\hline OAR $V_{x}$ & VMAT \% (SD) & IMRT \% (SD) & TOMO \% (SD) & IMPT \% (SD) \\
\hline \multicolumn{5}{|c|}{ Bi \& ipsilateral hippocampus } \\
\hline$V_{5 G y}$ & $90.2(25.9)$ & $92.1(23.6)$ & $85.1(29.7)$ & $78.8(32.5)$ \\
\hline $\mathrm{V}_{10 \mathrm{~Gy}}$ & $79.4(34.8)$ & $81.8(31.9)$ & $75.4(38.5)$ & $73.7(37.6)$ \\
\hline $\mathrm{V}_{15 \mathrm{~Gy}}$ & $72.6(39.6)$ & $73.5(38.5)$ & $68.1(41.9)$ & $70.2(39.8)$ \\
\hline $\mathrm{V}_{20 \mathrm{~Gy}}$ & $65.7(41.3)$ & $67.5(39.7)$ & $63.5(43.3)$ & $66.6(41.0)$ \\
\hline$V_{25 G y}$ & $59.5(42.5)$ & $62.5(41.5)$ & $59.3(43.5)$ & $63.2(42.3)$ \\
\hline$V_{30 G y}$ & $55.0(43.7)$ & $58.3(42.4)$ & $55.7(43.7)$ & $60.3(43.3)$ \\
\hline$V_{35 G y}$ & $50.7(44.2)$ & $52.9(43.0)$ & $51.3(43.2)$ & $57.7(43.9)^{*}$ \\
\hline $\mathrm{V}_{40 \mathrm{~Gy}}$ & 46.5 (43.9) & $48.0(43.4)$ & $45.8(42.7)$ & $54.6(44.2)$ \\
\hline$V_{45 G y}$ & $41.7(42.8)$ & $42.8(42.5)$ & $41.0(42.4)$ & $49.9(43.9)^{*}$ \\
\hline$V_{50 G y}$ & $34.3(40.9)$ & $35.8(41.9)$ & $35.6(40.5)$ & $42.0(42.0)^{*}$ \\
\hline \multicolumn{5}{|c|}{ Contralateral hippocampus } \\
\hline$V_{5 G y}$ & $74.8(39.3)$ & $67.6(44.5)$ & $66.2(35.9)$ & $10.8(22.3)^{*}$ \\
\hline$V_{10 G y}$ & $42.4(42.5)$ & $46.4(44.5)$ & $14.1(22.7)^{*}$ & $6.2(16.1)^{*}$ \\
\hline$V_{15 G y}$ & $21.4(37.3)$ & $24.5(38.1)$ & $6.5(22.8)^{*}$ & $4.3(13.1)^{*}$ \\
\hline$V_{20 G y}$ & $11.0(26.8)$ & $15.3(33.1)$ & $5.2(22.3)^{*}$ & $3.1(10.9)^{*}$ \\
\hline$V_{25 G y}$ & $4.3(12.7)$ & $10.3(26.6)$ & $3.9(16.9)^{*}$ & $2.4(9.3)^{*}$ \\
\hline$V_{30 G y}$ & $1.5(5.6)$ & 2.7 (7.9) & $2.2(9.5)^{*}$ & $1.9(8.0)^{*}$ \\
\hline$V_{35 G y}$ & $1.0(4.5)$ & $1.0(4.5)$ & $1.5(6.7)^{*}$ & $1.5(6.6)^{*}$ \\
\hline$V_{40 G y}$ & $0.91(4.0)$ & $0.91(4.0)$ & $1.2(5.3)^{*}$ & $1.2(5.2)^{*}$ \\
\hline$V_{45 G y}$ & $0.8(3.5)$ & $0.83(3.6)^{*}$ & $0.95(4.1)^{*}$ & $0.9(3.9)^{*}$ \\
\hline$V_{50 G y}$ & $0.6(2.6)$ & $0.72(3.1)^{*}$ & $0.71(3.1)^{*}$ & $0.44(1.9)^{*}$ \\
\hline \multicolumn{5}{|c|}{ Cerebellum posterior } \\
\hline$V_{5 G y}$ & $28.7(32.1)$ & $37.0(35.8)^{*}$ & $31.9(34.1)^{*}$ & $19.0(23.4)$ \\
\hline$V_{10 G y}$ & $19.0(25.9)$ & $24.2(27.8)^{*}$ & $20.5(27.3)$ & $15.2(21.7)$ \\
\hline$V_{15 G y}$ & $13.0(21.9)$ & $16.2(23.9)^{*}$ & $13.7(22.0)$ & $12.8(20.5)$ \\
\hline$V_{20 G y}$ & $9.6(20.7)$ & $12.1(22.2)^{*}$ & $10.3(20.6)$ & $11.0(19.4)$ \\
\hline$V_{25 G y}$ & $7.9(20.3)$ & $9.8(21.2)^{*}$ & $8.4(19.8)$ & $9.6(18.4)^{*}$ \\
\hline$V_{30 G y}$ & $6.8(19.4)$ & $8.2(20.4)^{*}$ & 6.9 (18.4) & $8.3(17.4)^{*}$ \\
\hline$V_{35 G y}$ & $5.8(17.2)$ & $7.1(19.5)^{*}$ & $5.8(16.6)$ & $7.1(16.4)^{*}$ \\
\hline $\mathrm{V}_{40 \mathrm{~Gy}}$ & 4.7 (14.6) & $6.0(18.0)$ & 4.7 (14.5) & $5.9(15.2)^{*}$ \\
\hline $\mathrm{V}_{45 \mathrm{~Gy}}$ & 3.7 (12.1) & $4.8(15.2)$ & 3.7 (12.2) & $4.7(13.3)^{*}$ \\
\hline$V_{50 G y}$ & $2.8(9.8)$ & 3.6 (12.4) & $2.6(8.9)^{*}$ & $2.8(8.3)$ \\
\hline \multicolumn{5}{|l|}{ Brain } \\
\hline $\mathrm{V}_{5 \mathrm{~Gy}}$ & 84.7 (11.7) & $83.2(12.2)$ & $85.9(10.7)^{*}$ & $48.5(16.3)^{*}$ \\
\hline$V_{10 G y}$ & 78.3 (13.8) & $72.6(15.5)^{*}$ & 78.9 (12.9) & $44.2(15.7)^{*}$ \\
\hline$V_{15 G y}$ & $67.6(17.4)$ & $62.7(18.2)^{*}$ & $67.2(16.6)$ & $41.3(15.2)^{*}$ \\
\hline$V_{20 G y}$ & 56.1 (18.9) & $52.9(19.8)$ & $55.0(17.1)$ & $38.8(14.7)^{*}$ \\
\hline$V_{25 G y}$ & 46.7 (18.6) & 44.7 (18.7) & $45.6(16.4)$ & $36.6(14.2)^{*}$ \\
\hline$V_{30 G y}$ & 39.1 (17.1) & $38.2(16.8)$ & $38.6(15.0)$ & $34.3(13.5)^{*}$ \\
\hline$V_{35 G y}$ & $33.2(15.0)$ & $33.0(14.6)$ & $33.3(13.7)$ & 31.9 (12.8) \\
\hline $\mathrm{V}_{40 \mathrm{~Gy}}$ & $28.6(13.0)$ & $28.9(12.6)$ & $29.2(12.5)$ & $29.4(12.1)$ \\
\hline $\mathrm{V}_{45 \mathrm{~Gy}}$ & $24.9(11.2)$ & $25.6(11.1)^{*}$ & $25.6(11.1)$ & $26.2(11.0)^{*}$ \\
\hline$V_{50 G y}$ & $20.7(9.3)$ & $21.8(9.6)^{*}$ & $20.9(9.5)$ & $21.1(9.0)$ \\
\hline
\end{tabular}

$\mathrm{OAR}=$ organ at risk; $\mathrm{SD}=$ standard deviation; $\mathrm{V}_{\mathrm{xGy}}=$ the volume receiving $\times \mathrm{Gy}(\mathrm{RBE}) ;{ }^{*}=\mathrm{p}<0.02$. 


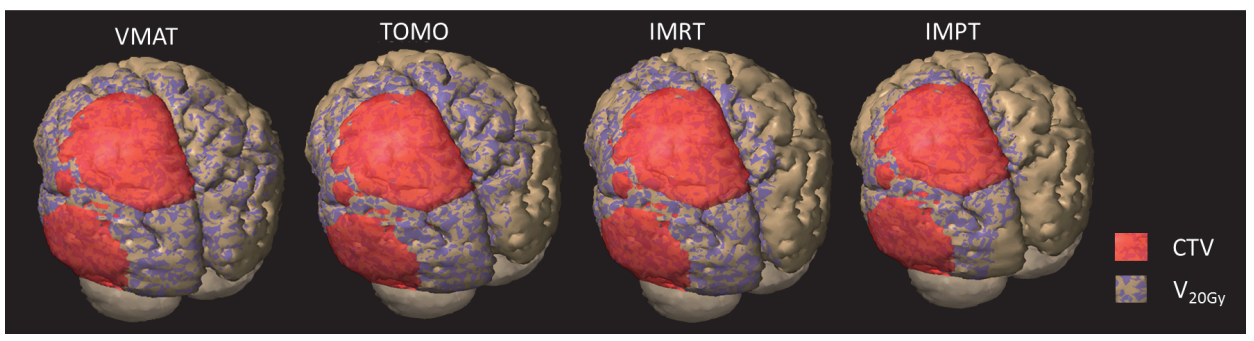

Figure 3.2 Three-dimensional representations of the cerebrum and cerebellum of a LGG patient highlighting the CTV in red and the $20 \mathrm{~Gy}$ isodose (purple/brown) of the different treatment techniques studied (VMAT, TOMO, IMRT and IMPT).

The mean $\mathrm{CTV}_{50.4 \mathrm{~Gy}}$ volume in the included 25 patients was $240 \mathrm{cc}$ (SD $112 \mathrm{cc}$ ) with a range of 92-456 cc and a median of $171 \mathrm{cc}$. With increasing volume of the $\mathrm{CTV}_{50.4 \mathrm{~Gy}}$, the volume of irradiated brain tissue increased for all treatment modalities. Even the slopes for all photon modalities (VMAT, IMRT, TOMO) were identical to that of the IMPT plans. The dose given to a certain volume, e.g., Brain $\mathrm{V}_{10 \mathrm{G} y}$, was lower with IMPT than with photon-based treatment modalities, whereas the curves for Brain $\mathrm{V}_{40 \mathrm{~Gy}}$ overlapped (Supplementary Figure S3.1).

\section{Discussion}

This ROCOCO study is the first to compare VMAT with IMRT, TOMO and IMPT in brain irradiation. IMPT was superior in sparing most OARs compared to VMAT and delivered the lowest integral dose. IMPT resulted in a significant dose reduction for structures related to cognition, such as non-target brain tissue, the hippocampus (bilateral and contralateral), and the posterior cerebellum. TOMO was the photon technique achieving the lowest dose to the OARs, while VMAT achieved the highest $\mathrm{Cl}$.

Thus far, there is limited experience comparing the different treatment techniques for LGG. Koca et al. ${ }^{22}$ published an IMRT versus TOMO plan comparison of 20 glioblastoma patients showing TOMO to be superior to IMRT plans in sparing of OARs with slightly broader low dose ranges. Cao et al. ${ }^{23}$ compared VMAT with TOMO for 10 body sites concluding comparable plan qualities of VMAT compared to TOMO in most of the cases. Skórska et al. ${ }^{24}$ stated in their retrospective plan comparison of 15 brain tumor patients, that the advantage of TOMO was in the highly conformal uniform doses to the target volume, even though these could only be delivered in a coplanar mode. 

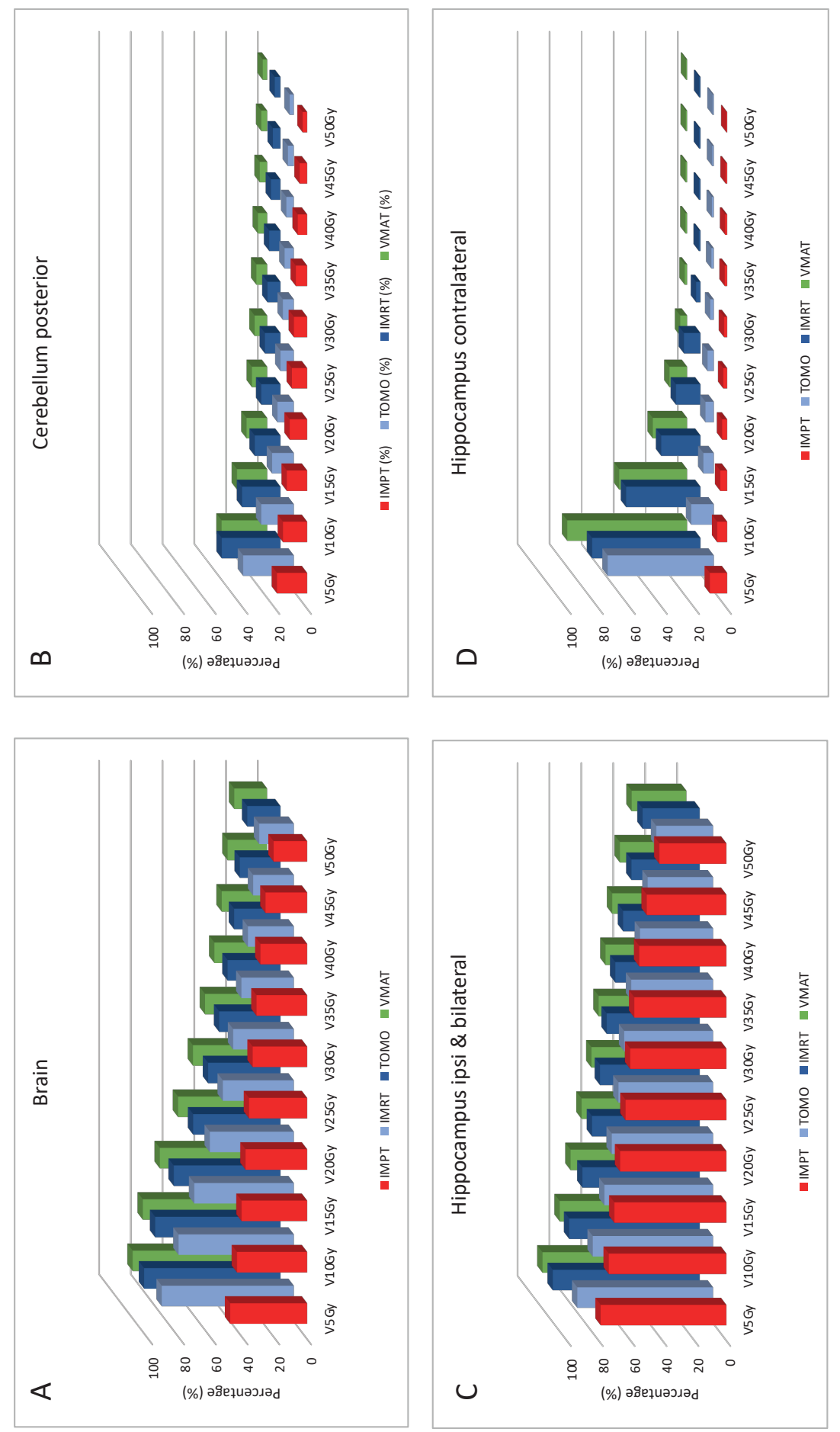

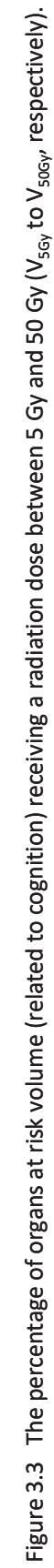


When comparing TOMO to coplanar (IMRT) and non-coplanar (ncIMRT) the median $\mathrm{Cl}$ were best for TOMO and worst for IMRT. They reported that the largest reduction of $D_{\text {max }}$ for lenses and $D_{\text {mean }}$ for both eyes was achieved using ncIMRT. While $D_{\text {mean }}$ for the optic chiasm and the ipsilateral optic nerve were best spared using TOMO, the contralateral optic nerve with ncIMRT $^{23}$.

Proton therapy has a dosimetric advantage due to its physical characteristics and is known to reduce the integral dose as was also seen in our in silico trial with a comparable coverage of the target volume and a lower dose to the OARs. Moreover, we found that in particular the dose to the contralaterally located OARs could be reduced. Thus far, publications on clinical experience treating LGG with proton therapy are scarce. Harrabi et $a l .{ }^{25}$ published the largest retrospective study on 74 LGG patients (median PTV volume $185 \mathrm{cc}$, range 12-710 cc) treated with proton therapy, generating a non state-of-the art conventional three-dimensional radiotherapy plan for plan comparison. The coverage of the target volume was comparable, also showing a reduction in maximal, mean, and integral doses to the OARs when using protons, especially in structures located contralaterally to the tumor. Other plan comparison studies concluded that proton therapy reduced the dose to surrounding normal tissues resulting in a significantly reduced whole-brain and -body irradiation ${ }^{26-28}$.

In our study the pituitary gland could impressively be spared using TOMO due to the planning strategy in which all direct photon fluence passing the pituitary gland was abolished using the features of the TOMO binary multileaf collimator ${ }^{29-31}$.

There are some shortcomings in our study, which may influence our results. First, all treatment plans were prepared in 3 different institutes, with their own routine and protocols besides the planning goals specifications which were prescribed by the study protocol. In line with international recommendations, e.g., the different PTV margins for each modality were determined locally based on local uncertainty data. However, this approach was chosen to include experts in their specific fields in this ROCOCO in silico study and, moreover, to reflect actual clinical practice. Second, one may debate about the required number of proton beams and their angles used in IMPT. There is an increasing awareness of the RBE uncertainty at the end of the Bragg peak, causing many proton centers to be cautious and avoiding overlapping spots at the end of the beam and using at least two beams ${ }^{32}$.

Third, treatment uncertainties (setup, range and anatomical uncertainties) might significantly influence the difference between planned and delivered dose as was shown by Kraan et al. ${ }^{33}$. It could also be argued that a smaller grid size in IMPT could slightly increase the maximum target dose, but will probably have no effect on the 
OARs as Rana et al. ${ }^{34}$ reported in their study varying the grid size from $1 \mathrm{~mm}$ to $3 \mathrm{~mm}$ for IMPT techniques. They recommend using a grid size of $2.5-3 \mathrm{~mm}$ for dose calculations with regard to the calculation time. Fourth, since there is currently no consensus on how to report robustness in a uniform manner, e.g. the suggestions of Liu et al. ${ }^{35}$, we only performed a small evaluation using the CV, fully aware of the fact that no absolute limit of this value is known. Further analysis based on a voxel wise minimum and maximum are still under investigation.

Whether proton therapy is indicated in neuro-oncology and outweighs the increased costs is still a matter of debate since no randomized control trials are published demonstrating a clinical benefit of proton therapy. Combs ${ }^{36}$ recently summarized and discussed the data on proton therapy for tumors of the CNS in comparison to modern photon therapy, showing that there are only few early data for low-grade glioma patients, underlining safety and low toxicity comparable to photons.

Will avoiding a low dose bath to the brain translate into a clinical benefit for the patient, especially regarding one of the most feared side effects: neurocognitive decline? This irreversible toxicity directly affects the patient's independence and wellbeing ${ }^{37}$. Variable mechanism can influence late cognitive toxicity to the brain related to radiotherapy such as vascular damage, demyelination and white matter changes as well as neurocognitive revalidation strategies ${ }^{37-41}$. Patients mostly exhibited overall stability in cognitive functioning after 5 years follow-up with, in some, more impairment on verbal measurements in tumors located in the left hemisphere and some on endocrine dysfunction. More data on late neurocognitive toxicity after PT as well as photon therapy is needed in relation to quality of life. Notably, the location of radiation dose deposition is considered important, defining type and level of radiation induced toxicity ${ }^{42}$. Moreover, it has to be considered that early side effects of proton and photon therapy for LGG are similar and transient, e.g., alopecia (81\%), dermatitis (78\%), fatigue (47\%), and headache (40\%), but bear the potential to adversely affect the patient's quality of life for several weeks ${ }^{43,44}$. Well-designed prospective studies including endpoints related to neurocognitive functioning and imaging are needed to determine the clinical relevance of low dose deposition to large CNS volumes.

In our study we did not correct for contrast enhancement. lodine contrast agents (CA) used during CT imaging, lead to an increase of the Hounsfield units in tissue with increased CA uptake depending on the CA concentration. This causes errors in the approximation of the tissue composition and thus in the calculation of the proton ranges as described by Wertz et $a .^{45}$. Consequently, this in theory leads to an exaggeration of the ion ranges during irradiation of $1.3 \mathrm{~mm}$, for a tumor with a size of $5 \mathrm{~cm}$. When OARs are close to the target volume this could be relevant ${ }^{45}$. Thus, the use of iodine contrast agent in a planning-CT to be used for proton therapy is highly 
discouraged. If urgently required in clinical practice, though, two sequential CT scans may be obtained, with the native CT scan being the first.

In order to predict the benefit of dose reduction to OARs, normal tissue complication probability (NTCP) models are needed. For cognition, Gondi et al. ${ }^{46}$ described a statistically significant correlation between the dose to $40 \%$ of both hippocampi $\left(D_{40 \%}\right)$ and cognitive decline in 18 patients with CNS tumors treated with stereotactic radiotherapy. This model needs validation in proton beam therapy and possible extension concerning the dose to each separate hippocampus, in particular when the ipsilateral hippocampus is part of the CTV.

Besides the hippocampi more regions in the brain related to neurocognition need to be identified, e.g., the posterior cerebellum. In our recent review (Eekers et al. ${ }^{47}$ ), we illustrated that there is growing evidence from structural and functional imaging studies that the cerebellum plays an eminent role in neurocognition and that radiation to the posterior cerebellum has a negative effect on neurocognitive outcomes in long-term pediatric brain survivors, besides the multimodality approach.

Since there is the problem of equipoise, the chance of ever performing a randomized trial, besides the costs of such a trial, seems very low. In the Netherlands, the modelbased approach has therefore been adopted by care givers and health insurance companies in order to provide an 'objective' tool to determine whether a patient is eligible for proton therapy ${ }^{48}$. In this model a $10 \%$ reduction in a grade 2 toxicity is needed using a validated (currently photon based) NTCP model. Unfortunately, there are currently no validated models for CNS. Therefore, uniform prospective collection of future toxicity data in a standardized way is urgently required for photon as well as proton therapy especially for low-dose-large-volume conditions. This will enable upfront assessment of a patient's likelihood to benefit from particle treatment. Uniform delineation and consensus on the tolerance on OARs are the first steps to achieve this besides a structured follow up including uniform neuro-cognitive tests ${ }^{18,49}$. This potentially improved quality of life ought to be outweighed against the additional costs of particles (protons, carbon ions) over technically advanced photon treatments. Changing the priorities as set in this study could alter outcome.

We conclude that IMPT can overall better spare organs than the other techniques, especially those OARs located contralateral to the target volume. In absence of NTCP models, an alternative approach, which will be implemented for supratentorial gliomas in The Netherlands, is to look at the reduction in mean dose to the brain and both hippocampi, excluding the CTV, achieved when using PT. However, the correlation of dose reduction to clinically relevant gain needs to be further investigated. Whether this will eventually lead to a significant improvement of quality of life needs to be determined in carefully designed future multicenter clinical studies. 


\section{References}

1. Crocetti E, Trama A, Stiller C, Caldarella A, Soffietti R, Jaal J, et al. Epidemiology of glial and non-glial brain tumours in Europe. Eur J Cancer. Eur J Cancer 2012;48(10):1532-42.

2. Eckel-Passow JE, Lachance DH, Molinaro AM, Walsh KM, Decker PA, Sicotte H, et al. Glioma Groups Based on 1p/19q, IDH, and TERT Promoter Mutations in Tumors. Cancer Res 2015;75(22):4884-94.

3. Brat DJ, Verhaak RG, Aldape KD, Yung WK, Salama SR, Cooper LA, et al. Cancer Genome Atlas Research Network. Comprehensive, Integrative Genomic Analysis of Diffuse Lower-Grade Gliomas. N Engl J Med 2015;372(26):2481-98.

4. Wesseling P, Capper D. WHO 2016 Classification of Gliomas. Neuropathol Appl Neurobiol. Neuropathol Appl Neurobiol 2018;44(2):139-50.

5. Van der Vlis TAMB, Hoeben A, Beckervordersandforth JC Ackermans L, Eekers DBP, Wennekes RMJ et al. Impact of the revised WHO classification of diffuse low-grade glioma on clinical decision making: A case report. Surg Neurol Int 2017;8:223.

6. Buckner JC, Shaw EG, Pugh SL, Chakravarti A, Gilbert MR, Barger GR, et al. Radiation plus Procarbazine, CCNU, and Vincristine in Low-Grade Glioma. N Engl J Med 2016;374(14):1344-55.

7. Edelstein K, Richard NM, Bernstein LJ. Neurocognitive impact of cranial radiation in adults with cancer: an update of recent findings. Curr Opin Support Palliat Care 2017;11(1):32-7.

8. Chakraborty S, Patil VM, Babu S Kumar SS, Ratheesan K, Bindu T, et al. Locoregional recurrences after post-operative volumetric modulated arc radiotherapy (VMAT) in oral cavity cancers in a resource constrained setting: experience and lessons learned. Br J Radiol 2015;88(1048):20140795.

9. Gondi V, Pugh SL, Tomé WA, Caine C, Corn B, Kanner A, et al. Preservation of memory with conformal avoidance of the hippocampal neural stem cell compartment during whole-brain radiotherapy for brain metastases (RTOG 0933): a phase 2 multi-institutional trial. J Clin Oncol 2014;32(34):3810-6.

10. Tian X, Liu K, Hou Y, Cheng J, Zhang J. The evolution of proton beam therapy: Current and future status. Mol Clin Oncol 2018;8(1):15-21.

11. Sakurai H, Ishikawa H, Okumura T. Proton beam therapy in Japan: current and future status.Jpn J Clin Oncol 2016;46(10):885-92.

12. Austin AM, Douglass MJJ, Nguyen GT, Penfold SN. A radiobiological Markov simulation tool for aiding decision making in proton therapy referral. Phys Med 2017;44:72-82.

13. Lühr A, von Neubeck C, Krause M, Troost EGC. Relative biological effectiveness in proton beam therapy Current knowledge and future challenges. Clin Transl Radiat Oncol 2018;9:35-41.

14. Eekers DB, Roelofs E, Jelen U Kirk M, Granzier M, Ammazzalorso F, et al. Benefit of particle therapy in re-irradiation of head and neck patients. Results of a multicentric in silico ROCOCO trial. Radiother Oncol 2016;121(3):387-94.

15. Van der Laan HP, van de Water TA, van Herpt HE, Christianen ME, Bijl HP, Korevaar EW, et al. Rococo cooperative group. The potential of intensity-modulated proton radiotherapy to reduce swallowing dysfunction in the treatment of head and neck cancer: A planning comparative study. Acta Oncol 2013;52(3):561-9.

16. Roelofs E, Engelsman M, Rasch C, Persoon L, Qamhiyeh S, de Ruysscher D, et al. ROCOCO Consortium. Results of a multicentric in silico clinical trial (ROCOCO): comparing radiotherapy with photons and protons for non-small cell lung cancer. J J Thorac Oncol 2012;7(1):165-76.

17. Eekers DBP, Roelofs E, Cubillos-Mesías C, Niël C, Smeenk RJ, Hoeben A, et al. Data from: Intensitymodulated proton therapy decreases dose to organs at risk in low-grade glioma patients: results of a multicentric in silico ROCOCO trial. CancerData 2018. doi:10.17195/candat.2018.09.1.

18. Eekers DBP, in 't Ven L, Roelofs E, Postma A, Alapetite C, Burnet NG, et al. The EPTN consensus-based atlas for CT- and MR-based contouring in Neuro-Oncology. Radiother Oncol 2018;128:(1):37-43.

19. Roelofs E, Persoon L, Qamhiyeh S, Verhaegen F, De Ruysscher D, Scholz M, et al. Design of and technical challenges involved in a framework for multicentric radiotherapy treatment planning studies. Radiother Oncol 2010;97(3):567-71. 
20. ICRU, 2010. ICRU. International Commission on Radiation Units and Measurements. Prescribing, recording, and reporting photon-beam intensity-modulated radiation therapy (IMRT). ICRU Report 83. J ICRU, 10 (2010):1-106.

21. Van 't Riet A, Mak AC, Moerland MA, Elders LH, van der Zee W, et al. A conformation number to quantify the degree of conformality in brachytherapy and external beam irradiation: Application to the Prostate. Int J Radiat Oncol Biol Phys 1997;37(3):731-6.

22. Koca $T$, Basaran $H$, Sezen D, Karaca S, Ors Y, Arslan D, Aydin A. Comparison of linear accelerator and helical tomotherapy plans for glioblastoma multiforme patients. Asian Pac J Cancer Prev 2014; 15(18):7811-6.

23. Cao D, Holmes TW, Afghan MK, Shepard DM. Comparison of plan quality provided by intensitymodulated arc therapy and helical tomotherapy. Int J Radiat Oncol Biol Phys 2007;69(1):240-50.

24. Skórska M, Piotrowski T, Kaźmierska J, Adamska K. A dosimetric comparison of IMRT versus helical tomotherapy for brain tumors. Phys Med 2014;30(4):497-502.

25. Harrabi SB, Bougatf N, Mohr A, Haberer T, Herfarth K, Combs SE, et al. Dosimetric advantages of proton therapy over conventional radiotherapy with photons in young patients and adults with low-grade glioma. Strahlenther Onkol 2016;192(11):759-69.

26. Dennis ER, Bussiere MR, Niemierko A, Lu MW, Fullerton BC, Loeffler JS, et al. A comparison of critical structure dose and toxicity risks in patients with low grade gliomas treated with IMRT versus proton radiation therapy Technol Cancer Res Treat 2013;12(1):1-9.

27. Schneider U, Lomax A, Pemler P, Lombriser N, Kaser-Hotz B. The impact of IMRT and proton radiotherapy on secondary cancer incidence. Int J Radiat Oncol Biol Phys 2007;68(3):892-7.

28. Beltran C, Roca M, Merchant TE. On the benefits and risks of proton therapy in pediatric raniopharyngioma. Int J Radiat Oncol Biol Phys 2012;82(2):e281-7.

29. Sherman JC, Colvin MK, Mancuso SM, Batchelor TT, Oh KS, Loeffler JS, et al. Neurocognitive effects of proton radiation therapy in adults with low-grade glioma. J J Neurooncol 2016;126(1):157-64.

30. Shih HA, Sherman JC, Nachtigall LB, Colvin MK, Fullerton BC, Daartz J, et al. Proton therapy for low-grade gliomas: results from a prospective trial. Cancer 2015;121(10):1712-9.

31. Brown PD, Buckner JC, Uhm JH, Shaw EG. The neurocognitive effects of radiation in adult low-grade glioma patients. Neuro Oncol 2003;5(3):161-7.

32. Lühr A, von Neubeck C, Krause M, Troost EGC. Relative biological effectiveness in proton beam therapy - Current knowledge and future challenges. Clin Transl Radiat Oncol 2018;9:35-41.

33. Kraan AC, van de Water S, Teguh DN, Al-Mamgani A, Madden T, Kooy HM, et al. Dose uncertainties in IMPT for oropharyngeal cancer in the presence of anatomical, range, and setup errors. Int J Radiat Oncol Biol Phys 2013;87(5):888-96.

34. Rana S, Zheng Y. Impact of grid size on uniform scanning and IMPT plans in XiO treatment planning system for brain cancer. J Appl Clin Med Phys 2015;16(5):447-56.

35. Liu W, Patel SH, Shen JJ, Hu Y, Harrington DP, Ding X, et al. Robustness quantification methods comparison in volumetric modulated arc therapy to treat head and neck cancer. Pract Radiat Oncol 2016;6(6):e269-75.

36. Combs SE. Does Proton Therapy Have a Future in CNS Tumors? Curr Treat Options Neurol 2017;19(3):12.

37. Klein M. Treatment options and neurocognitive outcome in patients with diffuse low-grade glioma. J Neurosurg Sci 2015;59(4):383-92.

38. Douw L, Klein M, Fagel SS, van den Heuvel J, Taphoorn MJ, Aaronson NK, et al. Cognitive and radiological effects of radiotherapy in patients with low-grade glioma: long-term follow-up. Lancet Neurol 2009; 8(9):810-8.

39. Armstrong CL, Hunter JV, Ledakis GE Cohen B, Tallent EM, Goldstein BH, et al. Late cognitive and radiographic changes related to radiotherapy: initial prospective findings. Neurology 2002;59(1):40-8.

40. Olson JD, Riedel E, DeAngelis L. Long-term outcome of low-grade oligodendroglioma and mixed glioma. Neurology 2000;54(7):1442-8.

41. Ravn Holmberg M, Sørensen P, Frøkjær JB, Carl J. Differences in supratentorial white matter diffusion after radiotherapy - new biomarker of normal brain tissue damage? Acta Oncol 2013;52(7):1314-9. 
42. Peiffer AM, Leyrer CM, Greene-Schloesser DM, Shing E, Kearns WT, Hinson WH, et al. Neuroanatomical target theory as a predictive model for radiation-induced cognitive decline. Neurology 2013;80(8): 747-53.

43. Hauswald H, Rieken S, Ecker S, Kessel KA, Herfarth K, Debus J, et al. First experiences in treatment of low-grade glioma grade I and II with proton therapy. Radiat Oncol 2012;7:189.

44. Wilkinson B, Morgan H, Gondi V, Larson GL, Hartsell WF, Laramore GE, et al. Low levels of acute toxicity associated with proton therapy for low-grade glioma: a Proton Collaborative Group study. Int J Radiat Oncol Biol Phys 2016;96(2S):E135.

45. Wertz $\mathrm{H}$, Jäkel $\mathrm{O}$. Influence of iodine contrast agent on the range of ion beams for radiotherapy. Med Phys 2004;31(4):767-73.

46. Gondi V, Hermann BP, Mehta MP, Tomé WA. Hippocampal dosimetry predicts neurocognitive function impairment after fractionated stereotactic radiotherapy for benign or low-grade adult brain tumors. Int J Radiat Oncol Biol Phys 2013;85(2):348-54.

47. Eekers DBP, in 't Ven L, Deprez S, Jacobi L, Roelofs E, Hoeben A, et al. The posterior cerebellum, a new organ at risk? Clin Transl Radiat Oncol 2017;8:22-26.

48. Langendijk JA, Lambin P, De Ruysscher D, Widder J, Bos M, Verheij, et al. Selection of patients for radiotherapy with protons aiming at reduction of side effects: the model-based approach. Radiother Oncol 2013;107(3):267-73.

49. Lambrecht M, Eekers DBP, Alapetite C, Burnet N, Calugaru V, Coremans IE, et al. Radiation dose constraints for organs at risk in neuro-oncology. Radiother Oncol 2018;128:26-36. 


\section{Supplemental material}

Table S3.1 Robustness analysis of the CTV for six randomly chosen patients.

\begin{tabular}{lccc}
\hline & \multicolumn{3}{c}{$\mathbf{V}_{\mathbf{9 5} \%}$ of $\mathbf{C T V}_{\mathbf{5 0 . 4 G y}}$} \\
\hline Patient & mean & SD & CV (\%) \\
1 & 99,95 & 0,05 & $0,05 \%$ \\
2 & 99,97 & 0,03 & $0,03 \%$ \\
3 & 99,94 & 0,05 & $0,05 \%$ \\
4 & 99,80 & 0,12 & $0,12 \%$ \\
5 & 99,95 & 0,03 & $0,03 \%$ \\
6 & 99,85 & 0,11 & $0,11 \%$ \\
\hline mean & 99,91 & 0,07 & $\mathbf{0 , 0 7 \%}$ \\
SD & 0,07 & 0,04 & $0,04 \%$ \\
\hline
\end{tabular}

$\mathrm{V}_{95 \%}=$ volume of the PTV receiving $95 \%$ of the prescribed dose, $\mathrm{CTV}_{50.4 \mathrm{G}}=$ clinical target volume with a prescribed dose of $50.4 \mathrm{~Gy}, \mathrm{SD}=$ standard deviation, CV = Coefficients of Variation, i.e., standard deviation over mean.
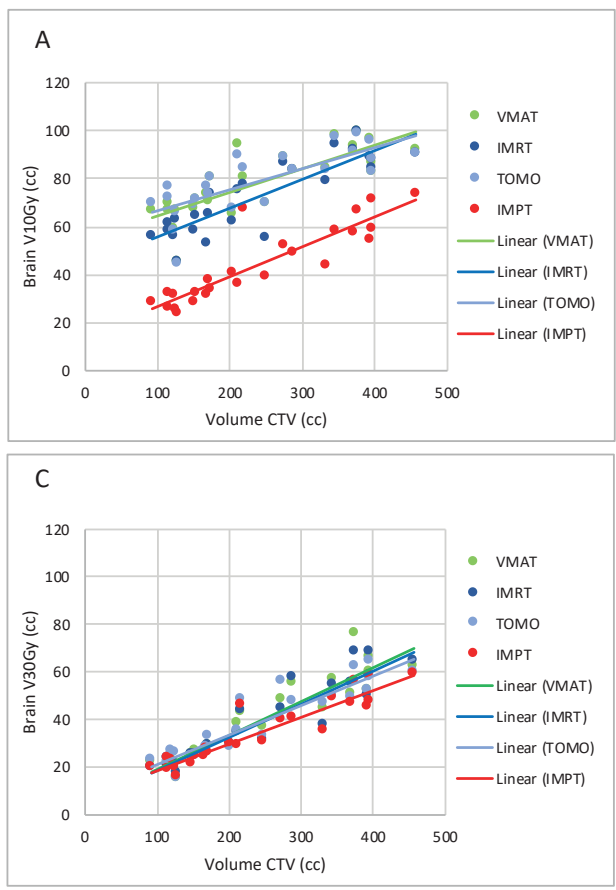
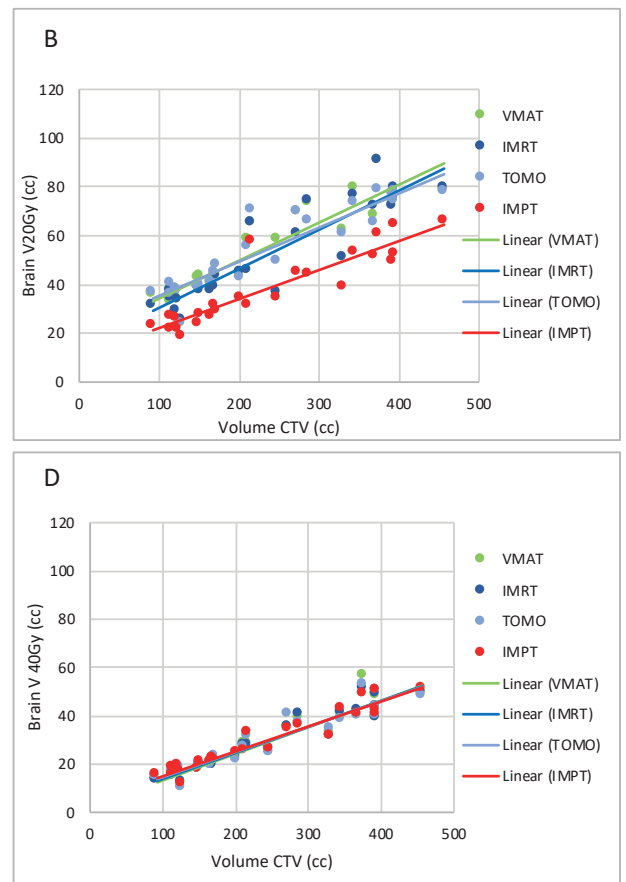

Figure S3.1 The volume irradiated brain (cc) per treatment modality (VMAT, IMRT, TOMO, IMPT) as a function of the CTV (cc) receiving a dose of (A) $10 \mathrm{~Gy}(\mathrm{RBE})$, (B) $20 \mathrm{~Gy}(\mathrm{RBE})$, (C) $30 \mathrm{~Gy}(\mathrm{RBE})$ and (D) $40 \mathrm{~Gy}(\mathrm{RBE})$; Brain $\mathrm{V}_{10 \mathrm{~Gy}}$ to $\mathrm{V}_{40 \mathrm{~Gy}}$, respectively. 



\section{Chapter 4}

The posterior cerebellum, a new organ at risk?

Daniëlle B.P.Eekers*, Lieke in 't Ven*, Sabine Deprez, Linda Jacobi, Erik Roelofs, Ann Hoeben, Philippe Lambin, Dirk de Ruysscher, Esther G.C. Troost

* equally shared first author

Clin Transl Radiat Oncol 2017;8:22-26 


\section{Abstract}

Eekers et al. ${ }^{1}$ have recently proposed a neuro-oncology atlas, which was co-authored by most centers associated in the European Proton Therapy Network (EPTN; Figure 4.1). With the introduction of new treatment techniques, such as integrated magnetic resonance imaging and linear accelerators (MR-linac) or particle therapy, the prediction of clinical efficacy of these more costly treatment modalities becomes more relevant. One of the side-effects of brain irradiation, being cognitive decline, is one of the toxicities most difficult to measure and predict. In order to validly compare different treatment modalities, 1) a uniform nomenclature of the organs at risk (OARs), 2) uniform atlas-based delineation [e.g., Eekers et al. ${ }^{1}$ ], 3) long-term follow-up data with standardized cognitive tests, 4) a large patient population, and 5) (thus derived) validated normal tissue complication probability (NTCP) models are mandatory.

Apart from the Gondi model ${ }^{2}$, in which the role of the dose to $40 \%$ of both hippocampi (HC) proves to be significantly related to cognition in 18 patients, no similar models are available. So there is a strong need for more NTCP models, on HC, brain tissue and possible other relevant brain structures.In this review we summarize the available evidence on the role of the posterior cerebellum as a possible new organ at risk for cognition, which is deemed relevant for irradiation of brain and head and neck tumors. 


\section{Introduction}

Survival rates of brain tumors, including gliomas have improved by the use of multimodality therapy, with advances in surgery, radiation therapy, chemotherapy and radiological technology prior to and throughout the treatment ${ }^{3,4}$. As survival rates increase, awareness of long-term complications due to therapy raises as well, for children as well as adults ${ }^{5}$. One of these (long-term) complications following (radio)therapy is neurocognitive decline, which is marked by reduction of verbal memory, spatial processing, attention and novel problem solving ability ${ }^{6}$. This decline has been shown in survivors of pediatric brain tumors, treated with multimodal treatment schedules, who have lower rates on high school graduation and employment relative to the overall population ${ }^{7}$.

Traditionally, the cerebral hemispheres are considered the regions of the brain responsible for cognitive function, while the cerebellum is known for its role in regulation and coordination in movement, posture and balance ${ }^{8}$. However, several clinical, anatomical and neuro-imaging studies have shown that the cerebellum may also play a role in neurocognition ${ }^{9-11}$. The aim of this review was to summarize the available evidence on role of the cerebellum in cognition and on the effects of radiation dose on the cerebellum in regard to neurocognitive function. Potentially, this will lead to new NTCP models, such as the Gondi model ${ }^{2}$, to predict neurocognitive outcomes related to radiation dose in different brain structures. Delineation guidelines for anatomical structures relevant in neuro-oncology have recently been proposed by Eekers et al. ${ }^{1}$ (Figure 4.1).
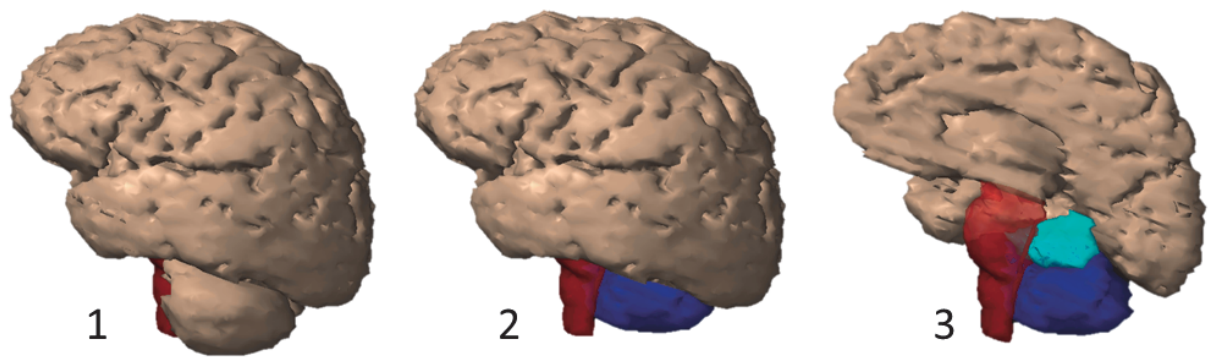

Figure 4.1 3D view of the Brain, brainstem, cerebellum posterior and anterior. Lateral view of a 3D-reconstruction on CT of: 1. Total brain (brown) and brainstem (red). 2. Brain (brown), brainstem (red), cerebellum posterior* (dark blue). 3. Right brain (brown), brainstem (red), right cerebellum anterior* (light blue) and right cerebellum posterior* (dark blue). ${ }^{*}$ In accordance to Atlas for Neuro-Oncology (Eekers et al. ${ }^{1}$ ). 


\section{Cognition and the cerebellum}

\section{Historical perspective}

Until the 20th century, studies on cerebellar function primarily focused on motor function ${ }^{12-14}$. It is unclear why cerebellar involvement in cognition and language remained uninvestigated in that period, but this may be due to the subtlety of cognitive defects or the fact that motor function and cognitive function were investigated as two separate entities. In 1971 Prescott and Piaget ${ }^{15}$ described that motor development is inherently connected to emotional and neurocognitive development. Children with motor development difficulties are often emotionally and cognitive challenged as well. Both development processes could be connected and cannot be studied separately. These new insights led to the theory that the cortex and cerebellum might be connected and that both regions may be involved in neurocognitive function. In 1978, Watson ${ }^{16}$ was one of the first authors to suggest the possible role of the cerebellum in sensory processing, learning, affect and cognition. In current literature, evidence is mounting to support this suggestion, even though many questions remain unanswered.

\section{Anatomy of the cerebellum}

The cerebellum consists of two hemispheres divided by the vermis. Both hemispheres are organized into ten lobules. Traditionally, the cerebellum has been recognized as having three anterior-posterior divisions ${ }^{10}$ : the primary fissure separates the anterior lobe (lobules I-V) from the posterior lobe (lobules VI-IX) and the posterolateral fissure separates the posterior lobe from the flocculonodular lobe (lobule $\mathrm{X}$ ). Two other approaches to divide the cerebellum are based upon functional $(F)$ or phylogenetic $(P)$ criteria. The vestibulocerebellum (F) or archicerebellum (P) contains the flocculonodular lobe and immediately adjacent vermis. The spinocerebellum (F) or paleocerebellum (P) contains the vermis and intermediate parts of the vermis. The cerebro-cerebellum (F) or neocerebellum (P) contains the lateral parts of the hemispheres ${ }^{10}$.

There are four deep nuclei in the cerebellum, the dentate, emboliform, globose, and fastigial, which receive and send information to the specific parts of the brain. Most afferent cerebral projections pass through the basal pontine nuclei and intermediate cerebellar peduncle, while most cerebello-cerebral efferent projections pass through dentate and ventral thalamic nuclei ${ }^{17,18}$. 


\section{Cerebellum and the sensorimotor \& associative cortex}

In order to determine whether the cerebellum plays a role in cognition it is crucial to unravel whether there are anatomical connections between the cerebellum and regions of the brain with higher cognitive functions. As a matter of fact, multiple studies, including viral tract tracing methods and resting-state functional connectivity data, support the presence of reciprocal links between the cerebellum and the prefrontal and parietal association cortices via cerebello-thalamo-cortical and corticoponto-cerebellar loops ${ }^{9-11,18-21}$. These closed loop circuits provide topographically segregated connections between the cerebral cortex and the cerebellum ${ }^{22,23}$. Information from the primary motor cortex passes the caudal part of the brainstem and enters the anterior part of the cerebellum via pontocerebellar fibers through the intermediate peduncle. Information from associative cortices passes several points in the brain stem and enters the posterior part of the cerebellum via the intermediate peduncle $^{21}$. In conclusion, the primary motor cortex is predominantly connected to the anterior part of the cerebellum, whereas the associative cortices are predominantly connected to the posterior part of the cerebellum ${ }^{10}$.

\section{Cerebellum activation in cognitive tasks}

Activation of the cerebellum in cognitive tasks has been found in multiple functional imaging studies. It is present during language, working memory, visual spatial and executive functioning tasks. Each domain and its matching tests have different activation patterns. Language-related activity is focused in lateral and posterior cerebellar regions, while working memory and reading tasks activate bilateral regions of the cerebellar posterior lobe, mainly lobules VI and VII. Functional imaging of affective processing, executive functioning and spatial processing highlights lobules VI and VII of the posterior cerebellar lobe. One of the first studies to describe this connection was published by Kim et al. ${ }^{24}$ in 1994. The authors used magnetic resonance imaging (MRI) to examine the activation of the dentate nucleus of the cerebellum in seven healthy human volunteers during their attempts to solve a puzzle. All seven adult participants showed more bilateral activation of the dentate nucleus during more challenging neurocognitive tasks. Later on, multiple imaging studies followed and found similar results ${ }^{10}$. In general, cerebellar activation during cognitive tasks is found in conjunction with activation of prefrontal and parietal regions, supporting the concept that the cerebellum is part of these functional networks ${ }^{10,11}$. 


\section{Cerebellum and cognition}

Clinical evidence for cognitive functioning of the cerebellum can, e.g., be found in the clinical cerebellar cognitive affective syndrome (CCAS; Figure 4.2). CCAS is a condition, which leads to deficits in cognitive functioning resulting from cerebellar damage. This syndrome has been described in both children and adults. Affected domains are executive function, spatial cognition, working memory, language and affect ${ }^{25,26}$. Depending on the location of the lesion, CCAS can be present in the absence of cerebellar motor syndrome, which is a syndrome that affects motor functions. The lesions that are associated with CCAS are situated in the posterior lobe of the cerebellum. Schmahmann and Pandyat ${ }^{19}$ were the first to describe this syndrome after studying 20 adults with cerebellar lesions due to either neoplasms, or vascular or traumatic damage. They all showed deficits in multiple cognitive domains as described above whilst maintaining semantic and episodic memory and consciousness. The latter deems cerebral damage as cause for these deficits unlikely. Lesions of the anterior lobe of the cerebellum produced only minor changes in executive and visual-spatial functions in contrary to the lesions in the posterior lobe. The constellation of deficits is suggestive of disruption in neural circuits connecting the cerebellum to prefrontal, posterior parietal, superior temporal, and limbic cortices ${ }^{25,26}$.

Cuny et al. $^{27}$ recently published the cases of two siblings with small retrovermian arachnoid cysts. The 3-year-old children initially presented cerebellar signs and cognitive disorders with progressive worsening. Surgery was performed to relieve intracranial pressure in the posterior fossa. In both cases significant improvement was seen in the children's neurological and neuropsychological status during 3 years of follow-up.

Other clinical studies show specific neurocognitive domains can be affected by damage to specific regions of the cerebellum. It is reported that verbal expression impairments result from damage to the right cerebellar lobe, whereas spatial difficulties can arise from lesions in the left cerebellar lobe, damage to the midline vermis has been associated with deficits in social and affective processing ${ }^{10,11,25,26}$.

\section{Cerebellar volume and cognitive function}

A large body of literature supports the hypothesis that cerebellar volume decreases with increasing age. It has been suggested that this has its effect on neurocognitive function as well. Hoogendam et al. ${ }^{28}$ examined the correlation between cerebellar volume and neurocognitive function. They included 3745 individuals above the age of 45 years and found a minor non-significant relationship between larger cerebellar volume and better global cognition, executive function, information processing speed, 
memory and motor speed. Their findings support the notion that the cerebellar volume has an influence on decline of cognition in aging but it is not the predominant structure. Likewise, Weier et al. ${ }^{29}$ examined 28 pediatric-onset relapsing-remitting Multiple Sclerosis patients, comparing their cerebellar volumes to a control group and found that while the volumes did not differ between groups, posterior cerebellar lobe volume and infra-tentorial lesion volume accounted for extra variance on measures of information processing and vocabulary. Many other studies support these findings and underline the hypothesis that the cerebellum has a function in neurocognitive functioning ${ }^{30}$.

\section{Radiation to the cerebellum}

One of the most interesting studies on radiation to the cerebellum was published by Merchant et $a l^{31}$, separately delineating the cerebellum and dividing it into a posterior and anterior part according to the article of Schmahmann et al. ${ }^{32}$. Seventy-eight children with low-grade glioma were included, prior treatment with chemotherapy was allowed in this study and there was no limit for the interval first surgery until irradiation (54-59.4 Gy), baseline and serial evaluations were performed to assess cognitive outcomes ${ }^{31}$.

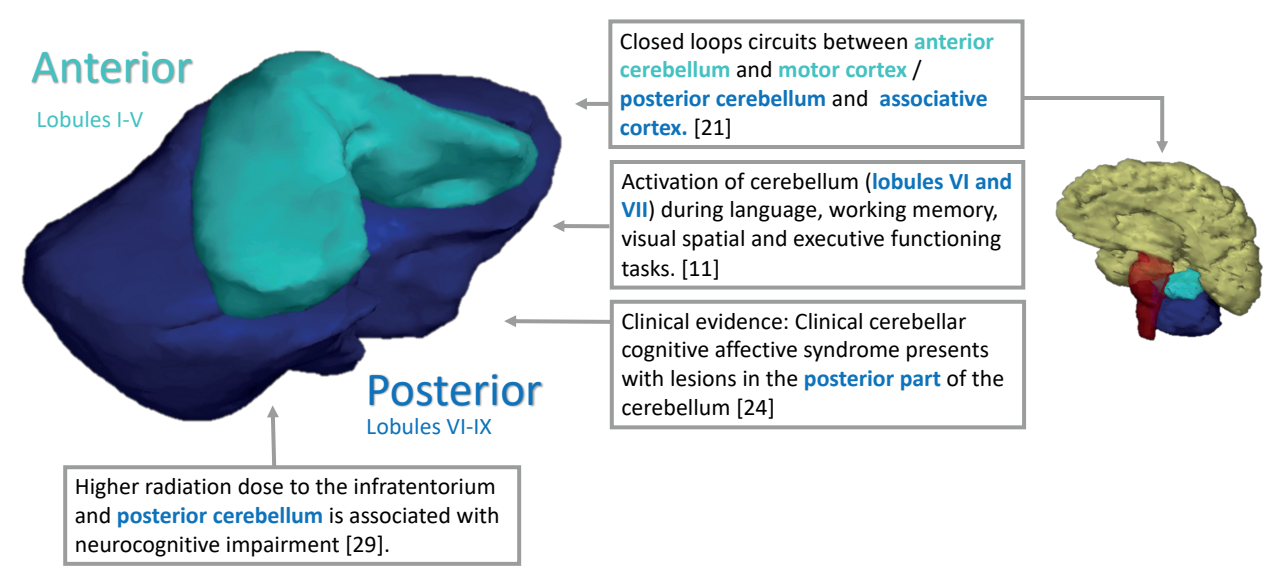

Figure 4.2 Role of cerebellum in cognition.

They found a statistically significant correlation between the radiation dose to the infratentorium and posterior cerebellum and neurocognitive impairment at several cognitive domains. To date, this is the only available study with separate dosimetric 
data for the posterior cerebellum. Noteworthy, one of the limitations of that study was the absence of a control group. Rønning et al..$^{33}$ compared an only surgically treated pediatric patient cohort with astrocytoma $(n=12)$ to a pediatric cohort with medulloblastoma $(n=10)$, who had been treated with surgery followed by radio(chemo)therapy. Both the astrocytoma and medulloblastoma groups scored below the standard norms regarding motor speed, attention and executive function. The medulloblastoma group, however, performed worse than the astrocytoma group on the following neuropsychological measures: intelligence, motor function, speed processing, verbal and visual memory. Since the astrocytoma group was treated with surgery alone, cerebellar lesions were held responsible for neurocognitive decline. The fact that the medulloblastoma cohort was more affected may be explained by several factors including the underlying malignancy and the use of radiotherapy ${ }^{33}$. Gan et al. ${ }^{34}$ assessed a group of ten adult patients treated with (intensity modulated) radio(chemo)therapy for squamous cell cancer of the head and neck. The authors delineated several brain structures separately, including temporal lobes and cerebellum, and performed neurocognitive function tests before and after treatment. The study population scored well on IQ but mean scores for all cognitive domains, except language and global cognitive function, were significantly lower than anticipated from the patients' IQ. Memory was the most severely affected cognitive domain. The patient with the lowest scores received a maximum dose of $36 \mathrm{~Gy}$ on the cerebellum and low radiation doses on the whole brain and hippocampi ${ }^{34}$. The studies of Merchant et $a . .^{49}$ even demonstrated that radiation dose-volume-parameters remain the most clinically significant determinants of IQ outcomes and that further reduction in radiation dose to specific volumes of the brain should be pursued.

Beside radiation dose to the cerebellum, multiple modalities in the treatment of brain tumor patients can induce neurocognitive sequelae. These include the pre-treatment neurocognitive function, surgery and peri-operative complications, radiation dose and volume to the craniospinal axis, systemic or intrathecal application chemotherapy, implantation of a shunt for increased intracranial pressure, patient factors such as age, stress, fatigue and anxiety ${ }^{33-49}$.

\section{Conclusion \& future perspectives}

There is growing evidence from structural and functional imaging studies that the cerebellum plays an evident role in neurocognition (Figure 4.2). Radiation to the posterior fossa has shown to have a negative effect on neurocognitive outcomes in long-term pediatric brain survivors. In order to derive an NTCP model for the (posterior) 
cerebellum, it is necessary to collect data on varying radiation doses to the (posterior and anterior) cerebellum and on prospectively assessing neurocognitive outcome. 


\section{References}

1. Eekers DBP, in 't Ven L, Roelofs E, Postma A, Alapetite C, Burnet N, et al. The EPTN consensus-based atlas for CT- and MR-based contouring in Neuro-Oncology. Radiother Oncol. 2018 Jul;128(1):37-43.

2. Gondi V, Hermann BP, Mehta MP, Tomé WA. Hippocampal dosimetry predicts neurocognitive function impairment after fractionated stereotactic radiotherapy for benign or low-grade adult brain tumors. Int J Radiat Oncol Biol Phys 2013; 85(2):348-54.

3. Kaatsch P. Epidemiology of childhood cancer. Cancer Treat Rev 2010;36(4):277-85.

4. Habrand JL, De Crevoisier R. Radiation therapy in the management of childhood brain tumors. Childs Nerv Syst 2001;17(3):121-33.

5. Ribi K, Relly C, Landolt MA, Alber FD, Boltshauser E, Grotzer MA. Outcome of medulloblastoma in children: Long-term complications and quality of life. Neuropediatrics 2005;36(6):357-65.

6. Greene-Schloesser D, Robbins ME. Radiation-induced cognitive impairment-from bench to bedside. Neuro-Oncol 2012;14 Suppl 4:iv37-44.

7. Hoppe-Hirsch E, Brunet L, Laroussinie F, Cinalli G, Pierre-Kahn A, Renier D et al. Intellectual outcome in children with malignant tumors of the posterior fossa: influence of the field of irradiation and quality of surgery. Childs Nerv Syst 1995;11(6):340-5.

8. Rapoport $M$, van Reekum $\mathrm{R}$, Mayberg $\mathrm{H}$. The role of the cerebellum in cognition and behavior: a selective review. J Neuropsychiatry Clin Neurosci 2000;12(2):193-8.

9. Leiner HC. Solving the mystery of the human cerebellum. Neuropsychol Rev 2010;20(3):229-35.

10. Stoodley CJ, Schmahmann JD. Evidence for topographic organization in the cerebellum of motor control versus cognitive and affective processing. Cortex J Devoted Study Nerv Syst Behav 2010;46(7):831-44.

11. Stoodley CJ, Valera EM, Schmahmann JD. Functional topography of the cerebellum for motor and cognitive tasks: an fMRI study. Neurolmage 2012;59(2):1560-70.

12. Fine EJ, Ionita CC, Lohr L. The history of the development of the cerebellar examination. Semin Neurol 2002;22(4):375-84.

13. Babinski JFF. Babinski sign. Arch Neurol Psychiatry 1933;29(1):168.

14. Holmes G. The cerebellum of man. Brain 1939;62(1):1-30.

15. Prescott JW. Early somatosensory deprivation as ontogenetic process in the abnormal development of the brain and behaviour. Medical Primatology 1970, pp. 356-375.

16. Watson PJ. Nonmotor functions of the cerebellum. Psychol Bull 1978;85(5):944-67.

17. Ristanović D, Milošević NT, Stefanović BD, Marić DL, Rajković K. Morphology and classification of large neurons in the adult human dentate nucleus: A qualitative and quantitative analysis of $2 \mathrm{D}$ images. Neurosci Res 2010; 67(1):1-7.

18. D'Angelo E, Casali S. Seeking a unified framework for cerebellar function and dysfunction: from circuit operations to cognition. Front Neural Circuits 2012; 6:116.

19. Schmahmann JD, Pandyat DN. The Cerebrocerebellar System. Int Rev Neurobiol 1997;41:31-60.

20. Middleton F. Basal ganglia and cerebellar loops: motor and cognitive circuits. Brain Res Rev 2000; 31(23):236-50.

21. Kelly RM, Strick PL. Cerebellar loops with motor cortex and prefrontal cortex of a nonhuman primate. J Neurosci Off J Soc Neurosci 2003;23(23):8432-44.

22. Bernard JA, Mittal VA. Dysfunctional Activation of the Cerebellum in Schizophrenia: A Functional Neuroimaging Meta-Analysis. Clin Psychol Sci J Assoc Psychol Sci 2015;3(4):545-66.

23. Noroozian M. The role of the cerebellum in cognition: beyond coordination in the central nervous system. Neurol Clin 2014;32(4):1081-104.

24. Kim SG, Uğurbil K, Strick PL. Activation of a cerebellar output nucleus during cognitive processing. Science, New Series, Vol. 265, No. 5174 (Aug. 12, 1994), pp. 949-951.

25. Schmahmann JD. The cerebellar cognitive affective syndrome. Brain 1998;121(4):561-79.

26. Schmamann JD, Caplan D. Cognition, emotion and the cerebellum. Brain 2006;129:290-2.

27. Cuny ML, Pallone M, Piana H, Boddaert N, Sainte-Rose C, Vaivre-Douret L, et al. Neuropsychological improvement after posterior fossa arachnoid cyst drainage. Childs Nerv Syst 2017;33(1):135-41. 
28. Hoogendam YY, van der Geest JN, Niessen WJ, van der Lugt A, Hofman A, Vernooij MW, et al. The role of cerebellar volume in cognition in the general elderly population. Alzheimer Dis Assoc Disord 2014; 28(4):352-7.

29. Weier K, Till C, Fonov V, Yeh EA, Arnold DL, Banwell B, et al. Contribution of the cerebellum to cognitive performance in children and adolescents with multiple sclerosis. Mult Scler J 2016;22(5):599-607.

30. Bernard JA, Seidler RD. Moving forward: age effects on the cerebellum underlie cognitive and motor declines. Neurosci Biobehav Rev 2014;42:193-207.

31. Merchant TE, Sharma S, Xiong $\mathrm{X}, \mathrm{Wu}$ S, Conklin $\mathrm{H}$. Effect of cerebellum radiation dosimetry on cognitive outcomes in children with infratentorial ependymoma. Int J Radiat Oncol Biol Phys 2014;90(3):547-53.

32. Schmahmann JD, Doyon J, McDonald D, Holmes C, Lavoie K, Hurwitz AS, et al. Three-dimensional MRI atlas of the human cerebellum in proportional stereotaxic space. Neuroimage 1999;10(3):233-60.

33. Rønning C, Sundet K, Due-Tønnessen B, Lundar T, Helseth E. Persistent cognitive dysfunction secondary to cerebellar injury in patients treated for posterior fossa tumors in childhood. Pediatr Neurosurg 2005;41(1):15-21.

34. Gan HK, Bernstein LJ, Brown J, Ringash J, Vakilha M, Wang L, et al. Cognitive functioning after radiotherapy or chemoradiotherapy for head-and-neck cancer. Int J Radiat Oncol Biol Phys 2011;81(1):126-34.

35. Brouwer CL, Steenbakkers RJHM, Bourhis J, Budach W, Grau C, Grégoire V, et al. CT-based delineation of organs at risk in the head and neck region: DAHANCA, EORTC, GORTEC, HKNPCSG, NCIC CTG, NCRI, NRG Oncology and TROG consensus guidelines. Radiother Oncol 2015;117(1):83-90.

36. Scoccianti S, Detti B, Gadda D, Greto D, Furfaro I, Meacci F, et al. Organs at risk in the brain and their dose-constraints in adults and in children: a radiation oncologist's guide for delineation in everyday practice. Radiother Oncol 2015;114(2):230-8.

37. Tamboli DA, Harris MA, Hogg JP, Realini T, Sivak-Callcott JA. Computed tomography dimensions of the lacrimal gland in normal caucasian orbits. Ophthal Plast Reconstr Surg 2011;27(6):453-6.

38. Celesia GG, DeMarco PJ. Anatomy and physiology of the visual system: J Clin Neurophysiol 1994; 11(5):482-92.

39. Merchant TE, Goloubeva O, Pritchard DL, Gaber MW, Xiong X, Danish RK, et al. Radiation dose-volume effects on growth hormone secretion. Int J Radiat Oncol 2002;52(5):1264-70.

40. Elson A, Bovi J, Kaur K, Maas D, Sinson G, Schultz C. Effect of treatment modality on the hypothalamicpituitary function of patients treated with radiation therapy for pituitary adenomas: hypothalamic dose and endocrine outcomes. Front Oncol 2014;4:73.

41. Rappaport R, Brauner R. Growth and endocrine disorders secondary to cranial irradiation. Pediatr Res 1989;25(6):561-7.

42. Baroncini $M$, Jissendi $P$, Balland $E$, Besson P, Pruvo J-P, Francke J-P, et al. MRI atlas of the human hypothalamus. Neurolmage 2012;59(1):168-80.

43. Chera BS, Amdur RJ, Patel P, Mendenhall WM. A radiation oncologist's guide to contouring the hippocampus. Am J Clin Oncol 2009;32(1):20-2.

44. Konrad C, Ukas T, Nebel C, Arolt V, Toga AW, Narr KL. Defining the human hippocampus in cerebral magnetic resonance images--an overview of current segmentation protocols. Neurolmage 2009; 47(4):1185-95.

45. Bender ET, Mehta MP, Tomé WAA. On the estimation of the location of the hippocampus in the context of hippocampal avoidance whole brain radiotherapy treatment planning. Technol Cancer Res Treat 2009;8(6):425-32.

46. Kojima S, Suzuki K, Hirata M, Shinohara H, Ueno E. Depicting the semicircular canals with inner-ear MRI: a comparison of the SPACE and TrueFISP sequences. J Magn Reson Imaging JMRI 2013;37(3):652-9.

47. Kocak-Uzel E, Gunn GB, Colen RR, Kantor ME, Mohamed ASR, Schoultz-Henley S, et al. Beam path toxicity in candidate organs-at-risk: assessment of radiation emetogenesis for patients receiving head and neck intensity modulated radiotherapy. Radiother Oncol 2014;111(2):281-8.

48. Sun Y, Yu X-L, Luo W, Lee AWM, Wee JTS, Lee N, et al. Recommendation for a contouring method and atlas of organs at risk in nasopharyngeal carcinoma patients receiving intensity-modulated radiotherapy. Radiother Oncol 2014;110(3):390-7. 
Chapter 4

49. Merchant TE, Kiehna EN, Li C, Xiong X, Mulhern RK. Radiation dosimetry predicts IQ after conformal radiation therapy in pediatric patients with localized ependymoma. Int J Radiat Oncol Biol Phys 2005; 63(5):1546-54. 


\section{Chapter 5 5}

\section{The EPTN consensus-based atlas for CT- and MR-based contouring in Neuro-Oncology}

Daniëlle B.P.Eekers, Lieke in 't Ven, Erik Roelofs, Alida Postma, Claire Alapetite, Neil G. Burnet, Valentin Calugaru, Inge Compter, Ida E.M. Coremans, Morton Høyer, Maarten Lambrecht, Alejandra Méndez Romero, Frank Paulsen, Ana Perpar, Dirk de Ruysscher, Laurette Renard, Pavel Vitek, Damien C. Weber, Hiske L. van der Weide, Gillian A. Whitfield, Ruud Wiggenraad, Petra Witt Nyström, Beate Timmermann, Esther G.C. Troost Radiother Oncol 2018;128(1):37-43 


\section{Abstract}

\section{Purpose}

To create a digital, online atlas for organs at risk (OAR) delineation in neuro-oncology based on high-quality computed tomography (CT) and magnetic resonance (MR) imaging.

\section{Methods}

CT and 3 Tesla (3T) MR images (slice thickness $1 \mathrm{~mm}$ with intravenous contrast agent) were obtained from the same patient and subsequently fused. In addition, a 7T MR without intravenous contrast agent was obtained from a healthy volunteer. Based on discussions between experienced radiation oncologists, the clinically relevant organs at risk (OARs) to be included in the atlas for neuro-oncology were determined, excluding typical head and neck OARs previously published. The draft atlas was delineated by a senior radiation oncologist, 2 residents in radiation oncology, and a senior neuroradiologist incorporating relevant available literature. The proposed atlas was then critically reviewed and discussed by European radiation oncologists until consensus was reached.

\section{Results}

The online atlas includes one CT-scan at two different window settings and one MR scan (3T) showing the OARs in axial, coronal and sagittal view. This manuscript presents the three-dimensional descriptions of the fifteen consensus OARs for neuro-oncology. Among these is a new OAR relevant for neuro-cognition, the posterior cerebellum (illustrated on 7T MR images).

\section{Conclusion}

In order to decrease inter- and intra-observer variability in delineating OARs relevant for neuro-oncology and thus derive consistent dosimetric data, we propose this atlas to be used in photon and particle therapy. The atlas is available online at www.cancerdata.org and will be updated whenever required. 


\section{Introduction}

In order to evaluate the added value of new radiotherapy (RT) modalities and techniques, such as particle therapy and adaptive highly conformal photon RT, it is essential to be able to accurately predict the individual patient's benefit in term of radiation-induced side effects ${ }^{1-3}$. The maturation and validation of normal tissue complication probability (NTCP) models is strongly dependent on uniform delineation of the relevant organs at risk (OARs), and reducing the inter- and intra-observer and trial protocol variability between clinicians and radiotherapy departments is an important objective. In this context, Brouwer et al. ${ }^{4}$ and Kong et al. ${ }^{5}$ published atlases for OARs relevant for head and neck and lung tumors, respectively.

During the last decade, several papers have been published on the delineation of OARs relevant to neuro-oncology both for adults and children ${ }^{4,6,7}$. These atlases may differ in minor details, but also some major discrepancies might occur, for instance, variations in the upper limit of the brainstem. Discrepancies in a critical OAR may influence the dose distribution and thus compromise the coverage of the target volume ${ }^{4,6}$.

Within the Dutch Platform for Neuro-Oncology and the ESTRO taskforce "European Particle Therapy Network (EPTN)" there was a need to generate an atlas, which identifies the relevant OARs for neuro-oncology and can be used both for daily practice as well as research purposes ${ }^{8}$. With the ever-growing insight into the influence of radiotherapy on neurological functions, it is essential that this atlas can be easily updated when indicated.

\section{Selection of OARs}

In order to avoid overlap with existing head and neck atlases, typical head and neck OARs, which were previously published, were excluded from this consensus atlas ${ }^{4}$. All OARs at present known to be relevant for radiation-induced toxicity in neuro-oncology were included, namely: brain, brainstem, cochlea, vestibulum \& semicircular canals, cornea, lens, retina, lacrimal gland, optic nerve, chiasm, pituitary, hippocampus and skin. In case of paired organs, each organ separately (left and right), and the unity of the two were contoured.

For future development of NTCP models, three distinct parts for the brainstem were defined, and regarding cognition, the posterior cerebellum, a new OAR possibly involved was included, as was the separation of the hippocampus into anterior and posterior parts. For research purposes also the hypothalamus was included. Of note, no 
validated dose response curve relationships have thus far been published for these separate parts of the brainstem, hippocampus and cerebellum.

\section{Uniform nomenclature}

To facilitate future comparison of the structures, the proposed nomenclature is in accordance with work by Santanam et al. ${ }^{9}$ on standardizing naming convention in radiation oncology, illustrated with quotes between brackets behind every structure name, for example: retina ("Retina_R", "Retina_L" and "Retinas").

\section{Delineation}

The fifteen OARs introduced in several previous publications were delineated by the first author $(D E)^{4,6,7}$. The anterior and posterior cerebellum were delineated by three authors (DE, LV, IC) using the high-resolution segment of the radiation treatment planning software Eclipse $^{\mathrm{TM}}$ v11.0 software, Varian, Palo Alto, CA). During a multidisciplinary session, the senior radiation oncologist (DE), neuro-radiologist (AP), and two residents in radiation oncology (LV, IC) discussed the delineation of the OARs and came to consensus on a first draft atlas. This draft was then critically reviewed by Dutch and international experts in neuro-oncology and consensus on the final version of this atlas was reached.

\section{Acquisition of $\mathrm{CT}$ and MR}

CT images were acquired with intravenous contrast (Ultravist ${ }^{\circledR}, 150 \mathrm{ml}$ of $300 \mathrm{mg}$ lodine per $\mathrm{ml}, 2 \mathrm{ml}$ per sec, $5 \mathrm{~min}$ delay, slice thickness $1 \mathrm{~mm}, 50 \mathrm{~cm}$ field of view, $120 \mathrm{kV}$, $685 \mathrm{mAs}$ ) using window-width/window-level settings (WW/WL) of 120/40 and 120/1500 (SOMATOM Sensation 10, Siemens Healthcare, Erlangen, Germany) of the head of an adult male low grade glioma patient after first resection. Moreover, a threedimensional spoiled gradient (3D-SPGR) axial 3T MR scan (1 mm slice thickness) scan of the same patient in standard axial, sagittal and coronal reconstruction, and an axial T2and a gadolinium (Gadovist ${ }^{\circledR} 1.0 \mathrm{mmol} / \mathrm{ml} 0.1 \mathrm{ml} / \mathrm{kg}$ bodyweight) contrast-enhanced axial T1-weighted sequence were acquired, with sagittal and coronal reconstruction. Both CT and MR were obtained in the supine position with the head in a neutral position; immobilization devices routinely used in radiation therapy were used for CT 
acquisition. Rigid MR-CT co-registration and delineation was performed using the Eclipse $^{\mathrm{TM}}$ treatment planning system with the high-resolution segment.

For illustration purposes, 7T MR images of a healthy volunteer were acquired (Siemens Magnetom 7T) with a slice thickness of $0.7 \mathrm{~mm}$ using a 32-channel head coil (Nova Medical Inc., Wilmington, CA; Figure 5.1). The magnetization-prepared rapid gradientecho (MP2RAGE) was selected for OAR delineation due to its superior soft tissue contrast (Figure 5.2). Scan parameters have previously been published by Compter et al. ${ }^{10}$. Vendor-based 3D distortion correction methods were applied.
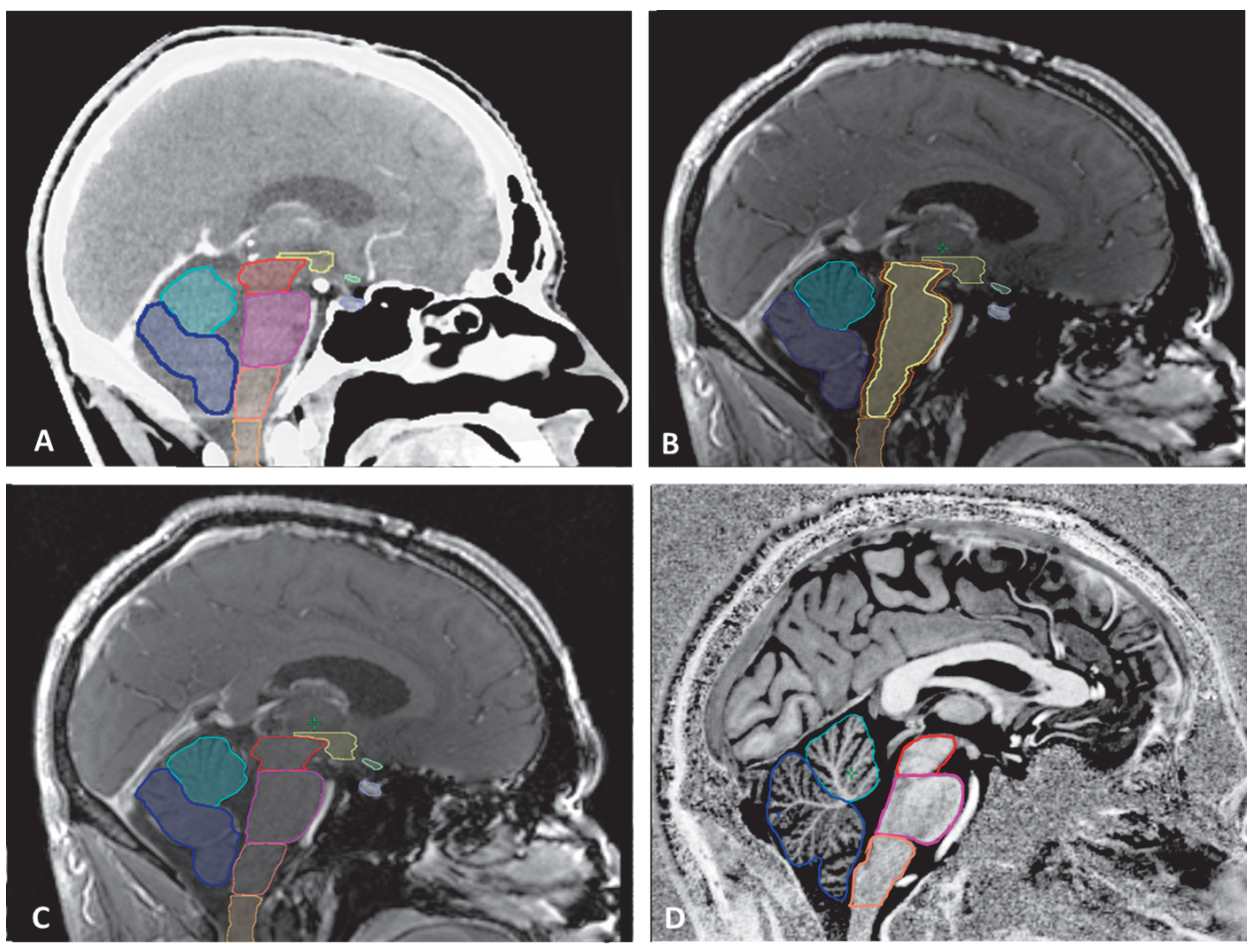

Figure 5.1 Sagittal (midline) view of the delineation

A: sagittal CT image (WL 140/40), B + C: sagittal 3 Tesla MRI (T1 with gadolinium), D: sagittal 7 Tesla MRI. Light blue = cerebellum anterior, dark blue = cerebellum posterior, red $=$ midbrain, magenta $=$ pons, pink = medulla oblongata, orange $=$ spinal cord, light yellow $=$ hypothalamus, green $=$ chiasm, purple $=$ pituitary, orange $=$ brainstem surface, yellow $=$ brainstem interior 


\section{Three-dimensional description of the OARs}

Cornea ("Cornea_R", "Cornea_L" and "Corneas")

The cornea is located at the anterior segment of the eyeball consisting of the structures ventral to the vitreous humor, the iris, ciliary body, and lens ${ }^{6}$. Using a brush of 2-3 mm the cornea can easily be delineated on MR as well as CT.

Retina ("Retina_R", "Retina_L" and "Retinas")

The retina is a neurosensorial membrane of 2-3 mm thickness, located at the posterior part of the eyeball, posterior to the cornea and lens, and is the innermost of the three layers that form the wall of the eyeball (sclera, uvea/choroid and retina). Using a $3 \mathrm{~mm}$ brush, it can be delineated on MR as well as CT as a membrane covering the posterior $5 / 6$ of the globe, extending nearly as far as the ciliary body. The anterior border of the retina is between the insertion of the medial rectus muscle and the lateral rectus muscle, posterior to the ciliary body. The optic nerve is excluded from this contour ${ }^{4,6}$.

Lacrimal gland ("LacrimalGland L", "LacrimalGland_R" and "LacrimalGlands")

The lacrimal gland is an almond shaped gland (18 $\mathrm{mm}$ craniocaudally, $15 \mathrm{~mm}$ axial length and $5 \mathrm{~mm}$ axial width) located in the orbit superior-lateral to the eye, superior to the lateral rectus muscle and lateral to the superior rectus muscle. It can be delineated on CT using soft brain 120/40 or soft tissue $350 / 50$ WW/WL settings ${ }^{4,6,11}$.

Lens of the Eye ("Lens_R", "Lens_L" and "Lenses")

The lens (diameter up to $10 \mathrm{~mm}$ ) is a clearly visible biconvex avascular structure, located between the vitreous humor and the iris and can easily be delineated on $\mathrm{CT}^{6}$. It should be taken into account that without instructing the patient, the position of the lens is not fixed and can vary during treatment.

Optic nerve ("OpticNerve_R", "OpticNerve_L" and "OpticNerves")

The optic nerve (2-5 mm thick) is delineated from the posterior edge of the eyeball, through the bony optic canal, where it narrows slightly, to the optic chiasm. Close to the optic chiasm, an MR scan (T1 weighted) is recommended for better delineation of the optic nerve. Contouring the optic nerve in continuity with the chiasm is crucial for dose reporting purposes, as dose gradients can be very steep with modern photon and proton techniques ${ }^{4,12}$. 


\section{Optic chiasm ("Chiasm")}

The optic chiasm (14 $\mathrm{mm}$ transverse, $8 \mathrm{~mm}$ antero-posterior and 2-5 $\mathrm{mm}$ thick) is located $1 \mathrm{~cm}$ superior to the pituitary gland, which has high signal on T1 MRI, and just anterior to the pituitary stalk (located above the sella turcica). The lateral border is the internal carotid artery. The chiasm is superiorly located in the antero-inferior part of the third ventricle, below the supra-optic recess and above the infundibular recess of the third ventricle, with the optic nerves in front and the divergence of the optic tracts behind. The anterior cerebral arteries and the anterior communicating artery are located ventral to the chiasm. A T1 weighted MR (axial, sagittal and coronal) is recommended for delineation of the optic chiasm ${ }^{4,6}$.

\section{Pituitary gland ("Pituitary")}

The pituitary gland cannot be easily identified on axial CT, although the bony margins of the fossa are well shown. It is oval-shaped (craniocaudally up to $12 \mathrm{~mm}$ ) and lies in the sella turcica. Laterally, the pituitary gland is bordered by the cavernous sinuses, which are well visible with intravenous contrast agent, it is just inferior to the brain, and is connected to the hypothalamus by its pituitary stalk. The borders of the pituitary gland can be defined best in the sagittal view ${ }^{4,5}$. Alternatively, the inner part of the sella turcica can be used as a surrogate anatomical bony structure best identified using bone 1500/950 or soft tissue 350/50 WL/WW on CT.

\section{Hypothalamus ("Hypothalamus_R, "Hypothalamus_L" and "Hypothalami")}

The hypothalamus $\left(2-4 \mathrm{~cm}^{3}\right)$ is a polygonal structure consisting of two separated volumes on each side of the third ventricle, delineated using MR-based anatomic landmarks representing surrogate boundaries for the hypothalamus itself. The superior boundaries are the axial slices containing the anterior and the posterior commissure. Inferiorly, the boundary consists of the base of the third ventricle or the visible edge of the cerebrospinal fluid (CSF) space within the suprasellar cistern, while posteriorly the contour reaches to the level of the interpeduncular fossa. The mammillary bodies should be included in the contour. The medial border consists of the third ventricle or the visible CSF space. Since the lateral border is not clearly visible, the contour was bounded laterally $3 \mathrm{~mm}$ from the third ventricle. Delineation on a T1 weighted MR is strongly recommended ${ }^{13-16}$.

Hippocampus ("Hippocampus_P_R", "Hippocampus_P_L", "Hippocampus_A_R", "Hippocampus_A_L", and "Hippocampi")

The literature describes considerable age- and disease-specific variability in hippocampal size (range $2.8-4.0 \mathrm{~cm}^{3}$ ) and location ${ }^{7,17-19}$. The hippocampus (HC) is 
delineated as the grey matter medial to the medial boundary of the temporal horn of the lateral ventricle, bordered medially by the quadrigeminal cistern as described by Gondi et al. ${ }^{7}$. Blum et al. ${ }^{20}$ suggest a separation of the $\mathrm{HC}$ into a posterior (corpus) and anterior (head) part using the lateral ventricle as dorsal border for the anterior hippocampus. In sagittal view, the head of the $\mathrm{HC}$ is separated from the body at the narrowing of the HC, with the uncus located dorsally ${ }^{20,21}$. Delineation on MR (T1 weighted) is essential.

Cochlea ("Cochlea_R", "Cochlea_L" and "Cochleas")

The cochlea is a spiral structure (up to $0.6 \mathrm{~cm}^{3}$ ) located in a bony cavity in the petrous portion of the temporal bone, caudal to the semicircular canals, lateral to the internal auditory canal. Using a WW/WL setting of 120/1500 on CT images, its volume can be defined as a small cavity. The structures of the inner ear are well visible on MR (T2 weighted) images ${ }^{4,6}$. The semicircular canals should not be included.

Vestibular and semicircular canal ("VSCC_R", "VSCC_L", "VSCCs")

As the semicircular canals are a part of the bony labyrinth with the superior, posterior and lateral canals aligned in three planes, delineation is advised using the bone setting on CT images (WW/WL 120/1500). The semicircular canal is located laterally and cranially of the cochlea. The canals are also visible as small cavities on MR (T2 weighted) ${ }^{6}$.

Brain Stem ("BrainStem", "Brainstem_surface", "Brainstem_interior" or "Midbrain", "Pons" and "Medulla Oblongata")

The brainstem is to be contoured on MR images and can be divided into three parts, from cranial to caudal, the midbrain, pons and medulla oblongata. The midbrain is defined from the nigral substance at the cerebral peduncle to the upper border of the pons. The pons is an oval shaped structure on sagittal views, which is easy to discriminate (see Figure 5.1C). The caudal limit of the medulla oblongata is the tip of the dens of C2 (i.e., the odontoid peg), which is also the cranial border of the spinal cord; the cranial limit is the ponto-medullary junction ${ }^{4,6,22}$. For practical reason the cerebral aquaduct is included until it becomes the $4^{\text {th }}$ ventricle. The brainstem interior is the brainstem surface contour cropped by $2 \mathrm{~mm}$ (inner border). The brainstem surface is the brainstem excluding the brainstem interior ${ }^{23,24}$. These structures are contoured automatically (and checked thereafter) using a built-in delineation tool commonly found in treatment planning systems. 
Brain ("Brain" and "Brain_Supratentorial")

The delineation of the brain includes the cerebellum, CSF and small brain vessels, and excludes the brainstem and large cerebellar vessels, such as the sigmoid sinus, transverse sinus and superior sagittal sinus (Figure 5.3C). For delineation purposes, CT in brain soft tissue 350/40 WW/WL-settings is recommended. Alternatively, the brain can be contoured automatically (and checked thereafter) using a built-in delineation tool commonly found in the majority of treatment planning systems ${ }^{4,6,25}$. In the middle cranial fossa the carotid canal and cavernous sinuses, most easily seen on contrastenhanced T1 MRI, should not be included. The supratentorial brain equals the "Brain" excluding the cerebellum (see Section Cerebellum ("Cerebellum_P", "Cerebellum_A" and "Cerebellum")).

\section{Cerebellum ("Cerebellum_P", "Cerebellum_A" and "Cerebellum")}

The separation of the cerebellum into an anterior and posterior part is best seen on the sagittal T1 weighted MR (see Figure 5.1). The anterior cerebellum consists of the cranial part of the cerebellum including half of the medullary corpus. The posterior cerebellum consists of the caudal and posterior part of the cerebellum with a cranial border including the lower half of the medullary corpus. This part includes the flocculonodular lobe. The primary fissure, which is best seen on7-Tesla MR (see Figure 5.2), divides the anterior part from the posterior part of the cerebellum [26]. The lateral borders for both parts are the large vessels (the sigmoid sinus, transverse sinus and superior sagittal sinus) and CSF, which are both excluded.
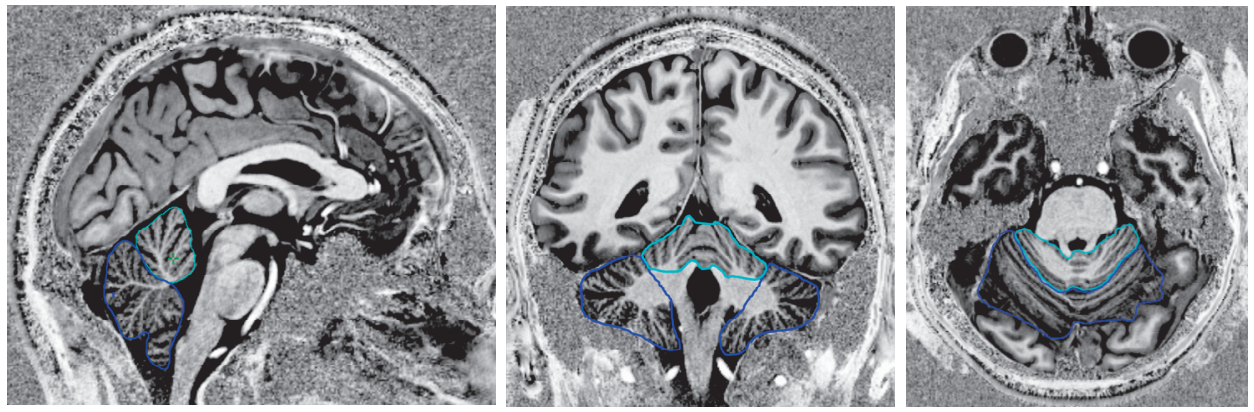

Figure 5.2 Sagittal (midline) view of cerebellum delineation on 7Tesla MRI

From left to right: 7 Tesla MRI, sagittal, coronal and transversal. Light blue = cerebellum anterior, dark blue $=$ cerebellum posterior

Skin ("Skin")

The skin is the volume defined by the body contour (outer border) and the body contour cropped by $5 \mathrm{~mm}$ (inner border), both created on the CT. This structure is 
contoured automatically (and checked thereafter) using a built-in delineation tool commonly found in treatment planning systems ${ }^{4,6,25}$.

All mentioned OARs are delineated on CT and MR (see Figure 5.3) in an easy accessible delineation atlas at www.cancerdata.org. ${ }^{27}$.
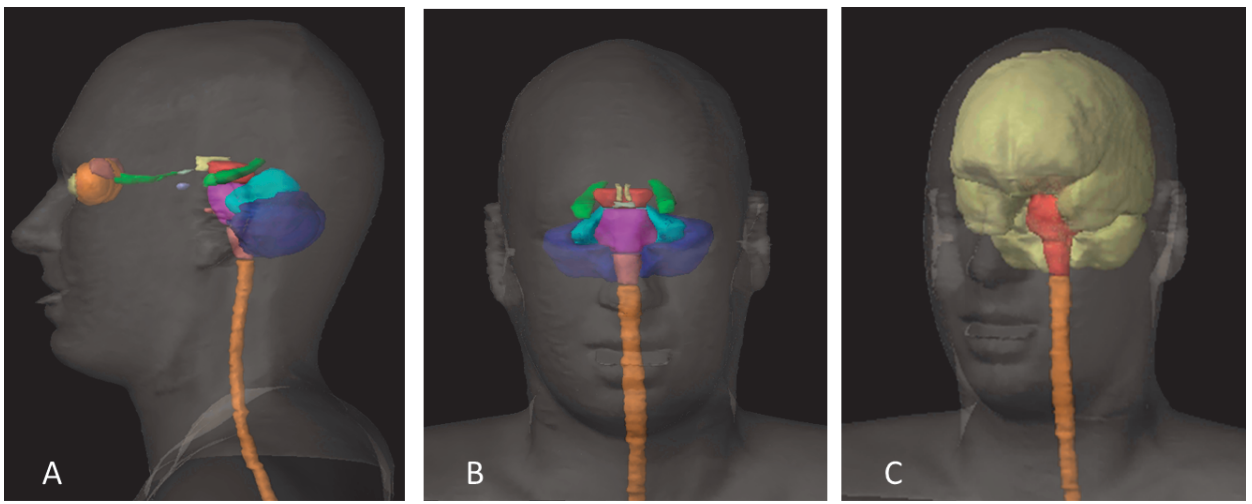

Figure 5.3 3D view of the OARs delineation on CT

A: From ventral to dorsal: yellow $=$ cornea, orange $=$ retina, brown = lacrimal gland, green $=$ optic nerve, light green $=$ chiasm, purple $=$ pituitary, yellow (central) $=$ hypothalamus, red $=$ midbrain, green (central) = hippocampus anterior, dark green = hippocampus posterior, pink = cochlea, magenta $=$ pons, pink $=$ medulla oblongata, orange $=$ spinal cord, light blue $=$ cerebellum anterior, dark blue $=$ cerebellum posterior. B: From cranial to caudal: yellow = hypothalamus, red = midbrain, light green = chiasm, green $=$ hippocampus anterior, dark green = hippocampus posterior, magenta $=$ pons, pink = medulla oblongata, Light blue = cerebellum anterior, dark blue $=$ cerebellum posterior, orange $=$ spinal cord. $\mathrm{C}$ : Yellow $=$ brain, red $=$ brainstem, orange $=$ spinal cord.

\section{Discussion}

The presented atlas for contouring OARs involved in neuro-oncology aims at reducing the inter- and intra-observer delineation variability and thus enabling more consistent plan comparison. This is especially relevant when comparing different radiation treatment techniques and modalities, and for establishing detailed dose-response relationships and NTCP models for different OARs.

Toxicity to the optical system is a feared complication especially when it results in partial or total loss of vision or pain to the eye. Despite their small volume, a separate delineation of the different optical structures is crucial in order to derive dose-volume histograms and predict post-radiation toxicity. The optic chiasm is an anatomically 
cross-shaped structure, as is depicted by Scoccianti et al. [6] and not round as presented in the atlas by Brouwer et al. ${ }^{4}$. Moreover, the chiasm should be contoured in continuity with both optic nerves in order to prevent high-dose deposits in undelineated voxels. The retina is to be delineated separately from the vitreous body, since radiation induced retinopathy can be treated if observed in an early state. Consequently, correct dose calculation is of utmost relevance in order to refer a patient suffering from this radiation induced side-effect ${ }^{28}$. Separate delineation of the cornea is proposed since toxicity and tolerance dose differ from that of the retina ${ }^{4,28,29}$. Damage to the cornea, radiation keratitis, is painful and deteriorates sight. The mean radiation dose to the lacrimal gland is related to the development of a dry eye, which can be painful and render the eye susceptible to infections ${ }^{28}$. A cataract can develop at a rather low dose to the lens. This side-effect can be alleviated by surgical implantation of an artificial lens ${ }^{28}$. A normal lens is well seen because of its high protein content, whereas an artificial lens is difficult to see on CT or MR, but since it tolerates radiation dose, delineation is not required.

Hypo-pituitarism may take a long time to be diagnosed since its symptoms can be vague. Depending on the mean dose to the pituitary gland, an early referral to an endocrinologist can facilitate early initiation of treatment and thus prevent impaired quality of life $\mathrm{e}^{30,31}$. In general, it is advised to delineate the entire sella content to be sure the whole pituitary is included rather than the central part only, excluding the suprasellar part of the infundibulum ${ }^{4,6}$. Even though there are no established dose constraints for the hypothalamus available yet, we believe that these data need to be collected in a prospective manner, in order to correlate levels of hormone production and regulation of metabolic processes with delivered radiation dose.

Hearing preservation after radiotherapy is known to be related to dose to the cochlea ${ }^{32-34}$. There is agreement in the literature on its delineation, most optimally done on a CT scan with thin slices using a bone WW/WL-setting considering its location in the mastoid bone ${ }^{4,6}$. Since dizziness is a side-effect occasionally reported after radiotherapy it was decided to delineate the semicircular canals as well, using the same WW/WL-settings as for the cochlea, although future data are needed to establish a validated dose constraint.

Recent literature has shown some dose response relationship between the hippocampus and cognition as described by Gondi et al. ${ }^{34}$ using the absolute radiation dose to $40 \%$ of both hippocampi (D40\%). We firmly encourage delineating the hippocampi separately and into anterior and posterior parts, since the left hippocampus is known to be dominant in most patients (including left-handed patients) for verbal memory, and the right hippocampus for non-verbal memory also known as 
visual memory. Moreover, the posterior parts of the hippocampi are more related to memory than the anterior parts ${ }^{35-39}$.

The brain is often automatically delineated and for practical reasons the included small vessels are left in the contour since editing the contour would be too time consuming. There are dose constraints for brain tissue, especially on high doses related to temporal lobe radionecrosis in head and neck cancer patients ${ }^{40-45}$. Regarding neuro-cognition, some publications on paediatric patients have shown a correlation between low dose radiation to the supra-tentorial brain and cognitive decline ${ }^{46,47}$. Further data are needed to transfer this knowledge to adult patients. Data on whole brain radiotherapy and prophylactic cranial irradiation have hinted at the negative effect of low dose on cognition ${ }^{48-55}$. However, there still is a strong need for an adult NTCP model on brain tissue and cognition.

Symptomatic brainstem necrosis is a feared, but rare complication following radiotherapy to the brain ${ }^{56,57}$. It was decided to contour the brainstem in three anatomically distinct parts, because some hypothesize that specific volumes within the brainstem are more sensitive to radiation than others. In particle beam therapy, the anterior surface and center of the brainstem are delineated separately since a higher tolerance at the surface of the brainstem has been observed ${ }^{23,24}$. This should be subject to further research for both photon and particle radiotherapy.

The delineation of the cerebellum is also added as a possible new OAR for research purposes, since there are data suggesting a relationship between the posterior cerebellum and cognition ${ }^{58}$. Cantelmi et al. $^{59}$ states that recognition of the important cognitive contributions of the cerebellum might lead to improved cognitive outcome and quality of life. This definitely needs further research into a possible dose response relationship and tolerance dose, which is only possible when agreement is reached in the delineation, as proposed based on Schmahmann et al. ${ }^{26,56,59}$.

For radiation treatment planning purposes, the skin is added as an OAR since alopecia and erythema are disturbing side-effects. Using the skin structure enables lowering the dose during treatment planning, which is of particular importance in proton therapy with its relatively high entry dose ${ }^{60}$.

A limitation of the atlas is the fact that the three dimensional angulation possibilities in a radiation treatment planning systems are often limited and patients are mostly not aligned perfectly in the midline. This is why the atlas was based on a random CT/MRdataset of an imperfectly aligned patient, resembling routine clinical practice. The atlas includes transversal, coronal and sagittal views to assist the delineation process. A second potential limitation of this atlas is that we had to limit the number of OARs proposed for otherwise the atlas would have been impracticable and thus not used in routine clinical practice. Future research should unravel the role of additional OARs and 
the NTCP value for: Eustachian tube, circle of Willis, optic tract, frontal \& temporal lobe, anterior eye chambers, macula, mammillary bodies, spinal canal and cerebrospinal space. Thirdly, this atlas does not summarize the available literature on dose constraints for the contoured organs at risk; this enormous effort will be a separate project of the EPTN. Finally, even though this atlas was contoured on a 3T MR scan, it can be easily transferred to $1.5 \mathrm{~T}$ MR images.

Uniform contouring of structures in the central nervous system, both in photon and particle therapy, is considered important for: 1) the generalization of normal tissue dose constraints, 2) establishment or update of NTCP models taking into account new radiation treatment techniques, and 3 ) for comparative multicenter clinical studies on radiotherapy in patients with primary brain tumors. Besides uniform contouring, consensus on OAR dose constraints is also required for implementing and improving NTCP models. A separate article on dose constraints for the given OARs, again consented by the EPTN, is currently being prepared.

\section{Conclusion}

In order to decrease variability in delineating OARs involved in neuro-oncology and to allow the generation of consistent dosimetric data, we propose an atlas for neurooncology including some new OARs in order to give an anatomical basis for the development of international acknowledged constraints and volumes. This will enable the community to amplify existing and new NTCP models such that more accurate prediction, and possibly prevention, of long-term radiation toxicity comes within reach. The atlas is available online on www.cancerdata.org and will be updated whenever required. 


\section{References}

1. Eekers DB, Roelofs E, Jelen U, Kirk M, Granzier M, Ammazzalorso F, Ahn PH, Janssens GO, Hoebers FJ, Friedmann T, Solberg T, Walsh S, Troost EG, Kaanders JH, Lambin P. Benefit of particle therapy in reirradiation of head and neck patients. Results of a multicentric in silico ROCOCO trial. Radiother Oncol 2016;121(3):387-94.

2. Roelofs E, Engelsman M, Rasch C, Persoon L, Qamhiyeh S, de Ruysscher D, Verhaegen F, PijlsJohannesma M, Lambin P; ROCOCO Consortium. Results of a multicentric in silico clinical trial (ROCOCO): comparing radiotherapy with photons and protons for non-small cell lung cancer. J Thorac Oncol 2012;7(1):165-76.

3. van der Laan HP, van de Water TA, van Herpt HE, Christianen ME, Bijl HP, Korevaar EW, Rasch CR, van 't Veld AA, van der Schaaf A, Schilstra C, Langendijk JA; Rococo cooperative group. The potential of intensity-modulated proton radiotherapy to reduce swallowing dysfunction in the treatment of head and neck cancer: A planning comparative study. Acta Oncol 2013;52(3):561-9.

4. Brouwer CL, Steenbakkers RJ, Bourhis J, Budach W, Grau C, Grégoire V, van Herk M, Lee A, Maingon P, Nutting C, O'Sullivan B, Porceddu SV, Rosenthal DI, Sijtsema NM, Langendijk JA. CT-based delineation of organs at risk in the head and neck region: DAHANCA, EORTC, GORTEC, HKNPCSG, NCIC CTG, NCRI, NRG Oncology and TROG consensus guidelines. Radiother Oncol 2015;117(1):83-90.

5. Kong FM, Ritter T, Quint DJ, Senan S, Gaspar LE, Komaki RU, Hurkmans CW, Timmerman R, Bezjak A, Bradley JD, Movsas B, Marsh L, Okunieff P, Choy H, Curran WJ Jr. Consideration of dose limits for organs at risk of thoracic radiotherapy: atlas for lung, proximal bronchial tree, esophagus, spinal cord, ribs, and brachial plexus. Int J Radiat Oncol Biol Phys 2011;81(5):1442-57.

6. Scoccianti S, Detti B, Gadda D, Greto D, Furfaro I, Meacci F, Simontacchi G, Di Brina L, Bonomo P, Giacomelli I, Meattini I, Mangoni M, Cappelli S, Cassani S, Talamonti C, Bordi L, Livi L. Organs at risk in the brain and their dose-constraints in adults and in children: a radiation oncologist's guide for delineation in everyday practice. Radiother Oncol 2015;114(2):230-8.

7. Gondi V, Tolakanahalli R, Mehta MP, Tewatia D, Rowley H, Kuo JS, Khuntia D, Tomé WA. Hippocampalsparing whole-brain radiotherapy: A "howto" technique, utilizing helical tomotherapy and LINAC-based intensity modulated radiotherapy. Int J Radiat Oncol Biol Phys 2010;78(4):1244-52.

8. Weber DC, Abrunhosa-Branquinho A, Bolsi A, Kacperek A, Dendale R, Geismar D, Bachtiary B, Hall A, Heufelder J, Herfarth K, Debus J, Amichetti M, Krause M, Orecchia R, Vondracek V, Thariat J, Kajdrowicz T, Nilsson K, Grau C. Profile of European proton and carbon ion therapy centers assessed by the EORTC facility questionnaire. Radiother Oncol 2017;124(2):185-9.

9. Santanam L, Hurkmans C, Mutic S, van Vliet-Vroegindeweij C, Brame S, Straube W, Galvin J, Tripuraneni $\mathrm{P}$, Michalski J, Bosch W. Standardizing naming conventions in radiation oncology. Int J Radiat Oncol Biol Phys 2012;83(4):1344-9.

10. Compter I, Peerlings J, Eekers DB, Postma AA, Ivanov D, Wiggins CJ, Kubben P, Küsters B, Wesseling P, Ackermans L, Schijns OE, Lambin P, Hoffmann AL. Technical feasibility of integrating $7 \mathrm{~T}$ anatomical MRI in image-guided radiotherapy of glioblastoma: a preparatory study. MAGMA 2016;29(3):591-603.

11. Bulbul E, Yazici A, Yanik B, Yazici H, Demirpolat G. Evaluation of Lacrimal Gland Dimensions and Volume in Turkish Population with Computed Tomography. J Clin Diagn Res 2016;10(2):TC06-8.

12. Celesia GG, DeMarco PJ Jr. Anatomy and physiology of the visual system. J Clin Neurophysiol 1994; 11(5):482-92. Review.

13. Merchant TE, Goloubeva O, Pritchard DL, Gaber MW, Xiong X, Danish RK, Lustig RH. Radiation dosevolume effects on growth hormone secretion. Int J Radiat Oncol Biol Phys 2002;52(5):1264-70.

14. Elson A, Bovi J, Kaur K, Maas D, Sinson G, Schultz C. Effect of treatment modality on the hypothalamicpituitary function of patients treated with radiation therapy for pituitary adenomas: hypothalamic dose and endocrine outcomes. Front Oncol 2014;4:73.

15. Rappaport R, Brauner R. Growth and endocrine disorders secondary to cranial irradiation. Pediatr Res 1989;25(6):561-7. Review.

16. Baroncini M, Jissendi P, Balland E, Besson P, Pruvo JP, Francke JP, Dewailly D, Blond S, Prevot V. MRI atlas of the human hypothalamus. Neuroimage 2012;59(1):168-80. 
17. Chera BS, Amdur RJ, Patel P, Mendenhall WM. A radiation oncologist's guide to contouring the hippocampus. Am J Clin Oncol 2009;32(1):20-2.

18. Konrad C, Ukas T, Nebel C, Arolt V, Toga AW, Narr KL. Defining the human hippocampus in cerebral magnetic resonance images. An overview of current segmentation protocols. Neuroimage 2009;47: 1185-95.

19. Bender ET, Mehta MP, Tomé WA. On the estimation of the location of the hippocampus in the context of hippocampal avoidance whole brain radiotherapy treatment planning. Technol Cancer Res Treat 2009;8:425-32.

20. Blum S, Habeck C, Steffener J, Razlighi Q, Stern Y. Functional connectivity of the posterior hippocampus is more dominant as we age. Cogn Neurosci 2014;5(0):150-9.

21. Sivakumar PT, Kalmady SV, Venkatasubramanian G, Bharath S, Reddy NN, Rao NP, Kovoor JM, Jain S, Varghese M. Volumetric analysis of hippocampal sub-regions in late onset depression: a 3 tesla magnetic resonance imaging study. Asian J Psychiatr 2015;13:38-43.

22. Kocak-Uzel E, Gunn GB, Colen RR, et al. Beam path toxicity in candidate organs-at-risk: Assessment of radiation emetogenesis for patients receiving head and neck intensity modulated radiotherapy. Radiother Oncol 2014;111:281-8.

23. Noël G, Habrand JL, Mammar H, Haie-Meder C, Pontvert D, Dederke S, Ferrand R, Beaudré A, Gaboriaud G, Boisserie G, Mazeron JJ. Highly conformal therapy using proton component in the management of meningiomas. Preliminary experience of the Centre de Protonthérapie d'Orsay. Strahlenther Onkol 2002; 178(9):480-5.

24. Feuvret L, Bracci S, Calugaru V, Bolle S, Mammar H, De Marzi L, Bresson D, Habrand JL, Mazeron JJ, Dendale R, Noël G. Efficacy and Safety of Adjuvant Proton Therapy Combined With Surgery for Chondrosarcoma of the Skull Base: A Retrospective, Population-Based Study. Int J Radiat Oncol Biol Phys 2016;95(1):312-21.

25. Sun $Y, Y u X-L$, Luo $W$, et al. Recommendation for a contouring method and atlas of organs at risk in nasopharyngeal carcinoma patients receiving intensity-modulated radiotherapy. Radiother Oncol 2014;110:390-7.

26. Schmahmann JD, Doyon J, McDonald D, Holmes C, Lavoie K, Hurwitz AS, Kabani N, Toga A, Evans A, Petrides $\mathrm{M}$. Three-dimensional MRI atlas of the human cerebellum in proportional stereotaxic space. Neuroimage 1999;10(3 Pt 1):233-60.

27. Eekers DBP, in 't Ven L, Roelofs E, Postma A, Troost EGC. EPTN International Neurological Contouring Atlas. CancerData. 2017. Doi: 10.17195/candat.2017.08.1.

28. Jeganathan VS, Wirth A, MacManus MP. Ocular risks from orbital and periorbital radiation therapy: a critical review. Int J Radiat Oncol Biol Phys 2011;79(3):650-9.

29. Barabino S, Raghavan A, Loeffler J, Dana R. Radiotherapy-induced ocular surface disease. Cornea 2005;24(8):909-14. Review.

30. De Marzi L, Feuvret L, Boulé T, Habrand JL, Martin F, Calugaru V, Fournier-Bidoz N, Ferrand R, Mazal A. Use of gEUD for predicting ear and pituitary gland damage following proton and photon radiation therapy. Br J Radiol 2015;88(1048):20140413.

31. Darzy KH, Shalet SM. Hypopituitarism following Radiotherapy Revisited. Endocr Dev 2009;15:1-24.

32. Bhandare N, Jackson A, Eisbruch A, Pan CC, Flickinger JC, Antonelli P, Mendenhall WM. Radiation therapy and hearing loss. Int J Radiat Oncol Biol Phys 2010;76(3 Suppl):S50-7.

33. Mujica-Mota M, Waissbluth S, Daniel SJ. Characteristics of radiation-induced sensorineural hearing loss in head and neck cancer: a systematic review. Head Neck 2013;35(11):1662-8.

34. Gondi V, Hermann BP, Mehta MP, Tomé WA. Hippocampal dosimetry predicts neurocognitive function impairment after fractionated stereotactic radiotherapy for benign or low-grade adult brain tumors. Int J Radiat Oncol Biol Phys 2012;83(4):e487-93.

35. Greene-Schloesser D, Moore E, Robbins ME. Molecular pathways: radiation-induced cognitive impairment. Clin Cancer Res 2013;19(9):2294-300.

36. Tsai PF, Yang CC, Chuang CC, Huang TY, Wu YM, Pai PC, Tseng CK, Wu TH, Shen YL, Lin SY. Hippocampal dosimetry correlates with the change in neurocognitive function after hippocampal sparing during whole brain radiotherapy: a prospective study. Radiat Oncol 2015;10:253. 
37. Blum S, Habeck C, Steffener J, Razlighi Q, Stern Y. Functional connectivity of the posterior hippocampus is more dominant as we age. Cogn Neurosci 2014;5(3-4):150-9.

38. Poppenk J, Moscovitch M. A hippocampal marker of recollection memory ability among healthy young adults: contributions of posterior and anterior segments. Neuron 2011;72(6):931-7.

39. Lee AW, Foo W, Chappell R, Fowler JF, Sze WM, Poon YF, Law SC, Ng SH, O SK, Tung SY, Lau WH, Ho JH. Effect of time, dose, and fractionation on temporal lobe necrosis following radiotherapy for nasopharyngeal carcinoma. Int J Radiat Oncol Biol Phys 1998;40(1):35-42.

40. Armstrong GT, Jain N, Liu W, Merchant TE, Stovall M, Srivastava DK, Gurney JG, Packer RJ, Robison LL, Krull KR. Region-specific radiotherapy and neuropsychological outcomes in adult survivors of childhood CNS malignancies. Neuro Oncol 2010;12(11):1173-86.

41. Marks LB, Yorke ED, Jackson A, Ten Haken RK, Constine LS, Eisbruch A, Bentzen SM, Nam J, Deasy JO. Use of normal tissue complication probability models in the clinic. Int J Radiat Oncol Biol Phys 2010;76(3 Suppl):S10-9.

42. Su SF, Huang SM, Han F, Huang Y, Chen CY, Xiao WW, Sun XM, Lu TX. Analysis of dosimetric factors associated with temporal lobe necrosis (TLN) in patients with nasopharyngeal carcinoma (NPC) after intensity modulated radiotherapy. Radiat Oncol 2013;8:17.

43. Hsu YC, Wang LF, Lee KW, Ho KY, Huang CJ, Kuo WR. Cerebral radionecrosis in patients with nasopharyngeal carcinoma. Kaohsiung J Med Sci 2005;21(10):452-9.

44. Zeng L, Huang SM, Tian YM, Sun XM, Han F, Lu TX, Deng XW. Normal Tissue Complication Probability Model for Radiation-induced Temporal Lobe Injury after Intensity-modulated Radiation Therapy for Nasopharyngeal Carcinoma. Radiology 2015;276(1):243-9.

45. Mulhern RK, Fairclough D, Ochs J. A prospective comparison of neuropsychologic performance of children surviving leukemia who received 18-Gy, 24-Gy, or no cranial irradiation. J Clin Oncol 1991;9(8):1348-56. Erratum in: J Clin Oncol 1991 Oct;9(10):1922.

46. Mulhern RK, Kepner JL, Thomas PR, Armstrong FD, Friedman HS, Kun LE. Neuropsychologic functioning of survivors of childhood medulloblastoma randomized to receive conventional or reduced-dose craniospinal irradiation: a Pediatric Oncology Group study. J Clin Oncol 1998;16(5):1723-8.

47. Armstrong GT, Jain N, Liu W, Merchant TE, Stovall M, Srivastava DK, Gurney JG, Packer RJ, Robison LL, Krull KR. Region-specific radiotherapy and neuropsychological outcomes in adult survivors of childhood CNS malignancies. Neuro Oncol 2010;12(11):1173-86.

48. Peiffer AM, Leyrer CM, Greene-Schloesser DM, Shing E, Kearns WT, Hinson WH, Tatter SB, Ip EH, Rapp SR, Robbins ME, Shaw EG, Chan MD. Neuroanatomical target theory as a predictive model for radiationinduced cognitive decline. Neurology 2013;80(8):747-53.

49. Ravn S, Holmberg M, Sørensen P, Frøkjær JB, Carl J. Differences in supratentorial white matter diffusion after radiotherapy--new biomarker of normal brain tissue damage? Acta Oncol 2013;52(7):1314-9.

50. Gregor A, Cull A, Traynor E, Stewart M, Lander F, Love S. Neuropsychometric evaluation of long-term survivors of adult brain tumours: relationship with tumour and treatment parameters. Radiother Oncol 1996;41(1):55-9.

51. Tallet AV, Azria D, Barlesi F, Spano JP, Carpentier AF, Gonçalves A, Metellus P. Neurocognitive function impairment after whole brain radiotherapy for brain metastases: actual assessment. Radiat Oncol 2012;7:77

52. Gan HK, Bernstein L, Brown J, Ringash J, Vakilha M, Wang L, Goldstein D, Kim J, Hope A, O'Sullivan B, Waldron J, Abdul Razak AR, Chen EX, Siu LL. Cognitive functioning after radiotherapy or chemoradiotherapy for head-and-neck cancer. Int J Radiat Oncol Biol Phys 2011;81(1):126-34.

53. Makale MT, McDonald CR, Hattangadi-Gluth JA, Kesari S. Mechanisms of radiotherapy-associated cognitive disability in patients with brain tumours. Nat Rev Neurol 2017;13(1):52-64.

54. Edelstein K, Richard NM, Bernstein $\amalg$. Neurocognitive impact of cranial radiation in adults with cancer: an update of recent findings. Curr Opin Support Palliat Care 2017;11(1):32-37.

55. Shih HA, Sherman JC, Nachtigall LB, Colvin MK, Fullerton BC, Daartz J, Winrich BK, Batchelor TT, Thornton LT, Mancuso SM, Saums MK, Oh KS, Curry WT, Loeffler JS, Yeap BY. Proton therapy for lowgrade gliomas: Results from a prospective trial. Cancer 2015;121(10):1712-9.

56. Mayo C, Yorke E, Merchant TE. Radiation associated brainstem injury. Int J Radiat Oncol Biol Phys 2010;76(3 Suppl):S36-41. 
57. DeSalvo MN. Radiation necrosis of the pons after radiotherapy for nasopharyngeal carcinoma: diagnosis and treatment. J Radiol Case Rep 2012;6(7):9-16.

58. Eekers DBP, 't Ven L, Deprez S, Jacobi L, Roelofs E, Hoeben A, Lambin P, de Ruysscher D, Troost EGC. The posterior cerebellum, a new organ at risk? Clin Transl Radiat Oncol 2017;8:22-26.

59. Cantelmi D, Schweizer TA, Cusimano MD. Role of the cerebellum in the neurocognitive sequelae of treatment of tumours of the posterior fossa: an update. Lancet Oncol 2008;9(6):569-76.

60. Whaley JT, Kirk M, Cengel K, McDonough J, Bekelman J, Christodouleas JP. Protective effect of transparent film dressing on proton therapy induced skin reactions. Radiat Oncol 2013;8:19. 



\section{Chapter $\mathbf{6}$}

\section{Radiation dose constraints for organs at risk in neuro-}

oncology; the European Particle Therapy

Network consensus

Maarten Lambrecht, Daniëlle B.P. Eekers, Neil G. Burnet, Valentin Calugaru, Ida E.M. Coremans, Piero Fossati, Morten Høyer, Alejandra Méndez Romero, Ana Perpar, Dirk de Ruysscher, Beate Timmermann, Pavel Vitek, Damien C. Weber, Hiske L. van der Weide, Gillian A. Whitfield, Ruud Wiggenraad, Erik Roelofs, Petra Witt Nyström, Esther G.C. Troost Radiother Oncol 2018;128(1):26-36 


\section{Abstract}

\section{Purpose}

For unbiased comparison of different radiation modalities and techniques, consensus on delineation of radiation sensitive organs at risk (OARs) and on their dose constraints is warranted. Following the publication of a digital, online atlas for OAR delineation in neuro-oncology by the same group, we assessed the brain OAR-dose constraints in a follow-up study.

\section{Methods}

We performed a comprehensive search to identify the current papers on OAR dose constraints for normofractionated photon and particle therapy in PubMed, Ovid Medline, Cochrane Library, Embase and Web of Science. Moreover, the included articles' reference lists were cross-checked for potential studies that met the inclusion criteria. Consensus was reached among 20 radiation oncology experts in the field of neuro-oncology.

\section{Results}

For the OARs published in the neuro-oncology literature, we summarized the available literature and recommended dose constraints associated with certain levels of normal tissue complication probability (NTCP) according to the recent ICRU recommendations. For those OARs with lacking or insufficient NTCP data, a proposal for effective and efficient data collection is given.

\section{Conclusion}

The use of the European Particle Therapy Network-consensus OAR dose constraints summarized in this article is recommended for the model-based approach comparing photon and proton beam irradiation as well as for prospective clinical trials including novel radiation techniques and/or modalities. 


\section{Introduction}

The field of radiotherapy is rapidly evolving with new techniques, e.g., MR-linac, and beam modalities, i.e., protons and carbon ions, entering the scene of image-guided high precision treatment. These innovations aim at increasing the tumour control probability (TCP) while maintaining or reducing the normal tissue complication probability (NTCP). For comparison of the latter, ideally, consensus on (1) the delineation of the organs at risk (OARs), on (2) the tolerable radiation dose to be administered to the OARs, and on (3) the outcome reporting measure, i.e., uniform follow-up timing, patient questionnaires and content of the follow-up, should exist.

Regarding the first pre-requisite, Eekers et $a l^{1,2}$ recently published a digital, online atlas for OAR delineation in neuro-oncology on behalf of the task group "European Particle Therapy Network" (EPTN) of ESTRO. Addressing the second required condition, it has been a while since the recommendations by Emami et $a l^{3}$ and the QUANTEC series ${ }^{4-7}$ were published. In an attempt to reach the ideal conditions for comparison, we therefore summarize the OAR's distinct radiation induced toxicities and the recommended dose constraints for conventionally fractionated radiotherapy.

Moreover, we identified gaps of knowledge that need to be filled, preferably in a prospective multi-centre effort, to fully exploit the potential of highly conformal radiotherapy. Of note, this summary of the literature does explicitly not cover hypofractionated / ablative regimens, carbon ion radiotherapy, re-irradiation, or paediatric data.

\section{Materials and methods}

For each of the OAR described in the EPTN delineation consensus paper a dose constraint was sought for and the available data summarized ${ }^{1}$. Published manuscripts were identified through a PubMed search using combinations of ("radiotherapy" or "radiation therapy" or "radiation-induced") and "xerophthalmia"; "dry eye syndrome"; "keratoconjunctivitis"; "retinopathy"; "cataracts"; "optic neuropathy"; "vision loss"; "hemianopsia"; "hearing loss"; "tinnitus"; "vertigo"; "hypopituitarism"; "neurocognition"; "radionecrosis"; "Temporal lobe necrosis"; "brain stem toxicity"; "hippocampus"; "cerebellum"; "alopecia". Those manuscripts available in English or French, containing data on adult patients obtained from primary conventionally fractionated photon and proton radiotherapy, and describing a dose-toxicity relationship were included in this recommendation. Papers on re-irradiation, 
hypofractionation, carbon ion therapy and stereotactic ablative radiotherapy were omitted.

Relevant papers were summarized and put into Supplementary Tables (6.1-6.10)

The relevant quantitative analyses of normal tissue effect in the clinic (QUANTEC) papers were used for reference when applicable as was the paper by Emami et al. ${ }^{3-7}$.

The literature was then reviewed by 20 Radiation Oncology experts in the field of neuro oncology and a consensus was reached as depicted in Table 6.1 (see Figure 6.1). The units of all dose constraints are given in Gy regardless of the reported unit in the analysed data. Doses were recalculated to equivalent dose in 2 Gy-fractions (EQD2) using the formula:

$E Q D_{2}=\frac{D(d+\alpha / \beta)}{(2+\alpha / \beta)}$ with $D:$ the total dose and d: the dose per fraction.

Table 6.1

\begin{tabular}{|c|c|c|c|}
\hline Organ & $\begin{array}{l}\alpha / \beta \\
(G y)\end{array}$ & $\begin{array}{l}\text { Dose constraint } \\
\text { EQD2 }\end{array}$ & Toxicity \\
\hline Brain $^{7,87-90}$ & 2 & $\mathrm{~V}_{60 \mathrm{~Gy}} \leq 3 \mathrm{cc}$ & Symptomatic brain necrosis \\
\hline Brainstem $^{52,93-101}$ & 2 & $\begin{array}{l}\text { Surface } D_{0.03 c c} \leq 60 \mathrm{~Gy} \\
\text { Interior } D_{0.03 c c} \leq 54 \mathrm{~Gy}\end{array}$ & Permanent cranial neuropathy or necrosis \\
\hline Chiasm \& Optic nerve ${ }^{23,48-54}$ & 2 & $\mathrm{D}_{0.03 c c} \leq 55 \mathrm{~Gy}$ & Optic neuropathy \\
\hline Cochlea ${ }^{57-60,64-66}$ & 3 & $\begin{array}{l}\mathrm{D}_{\text {mean }} \leq 45 \mathrm{~Gy} \\
\mathrm{D}_{\text {mean }} \leq 32 \mathrm{~Gy}\end{array}$ & $\begin{array}{l}\text { Hearing loss } \\
\text { Tinnitus }\end{array}$ \\
\hline Cornea $^{13,21}$ & 3 & $\mathrm{D}_{0.03 c c} \leq 50 \mathrm{~Gy}$ & Erosion/ulceration \\
\hline Hippocampus $s^{108,109}$ & 2 & $\mathrm{D}_{40} \% \leq 7.3 \mathrm{~Gy}$ & Memory loss \\
\hline Lacrimal gland ${ }^{9,11,14-16}$ & 3 & $D_{\text {mean }} \leq 25 \mathrm{~Gy}$ & Keratoconjunctivitis sicca \\
\hline Lens $^{36,37}$ & 1 & $\mathrm{D}_{0.03 c \mathrm{cc}} \leq 10 \mathrm{~Gy}$ & Cataract \\
\hline Pituitary $66,76,79,80$ & 2 & $\begin{array}{l}D_{\text {mean }} \leq 45 \mathrm{~Gy} \\
D_{\text {mean }} \leq 20 \mathrm{~Gy}\end{array}$ & $\begin{array}{l}\text { Panhypopituitarism } \\
\text { Growth hormone deficiency }\end{array}$ \\
\hline Retina $^{13,23,26,31}$ & 3 & $\mathrm{D}_{0.03 c c} \leq 45 \mathrm{~Gy}$ & Loss of vision \\
\hline Skin ${ }^{114}$ & 2 & $\mathrm{D}_{0.03 c c} \leq 25 \mathrm{~Gy}$ & Permanent alopecia \\
\hline
\end{tabular}

EQD2 = equivalent dose in 2 Gy per fraction; $D_{3} \mathrm{cc}=$ dose to $3 \mathrm{cc}$ of structure/organ; $\mathrm{D}_{0.03 \mathrm{cc}}=$ near maximum dose to $0.3 \mathrm{cc}$ of structure/organ; $\mathrm{D}_{\text {mean }}=$ mean dose; $\mathrm{D}_{40 \%}=$ mean dose to $40 \%$ of the volume of both hippocampi.

\section{Results}

\section{Orbital structures}

Radiotherapy of central nervous system (CNS) tumours often results in intentional or incidental irradiation of the different orbital structures. This gives rise to a wide variety of acute and late toxicities ranging from transient erythema of the peri-orbital skin to 
permanent blindness. The complex anatomy and physiology of the eye make it a challenging task to give a full and detailed description of all toxicities, and literature on many of them is scarce.

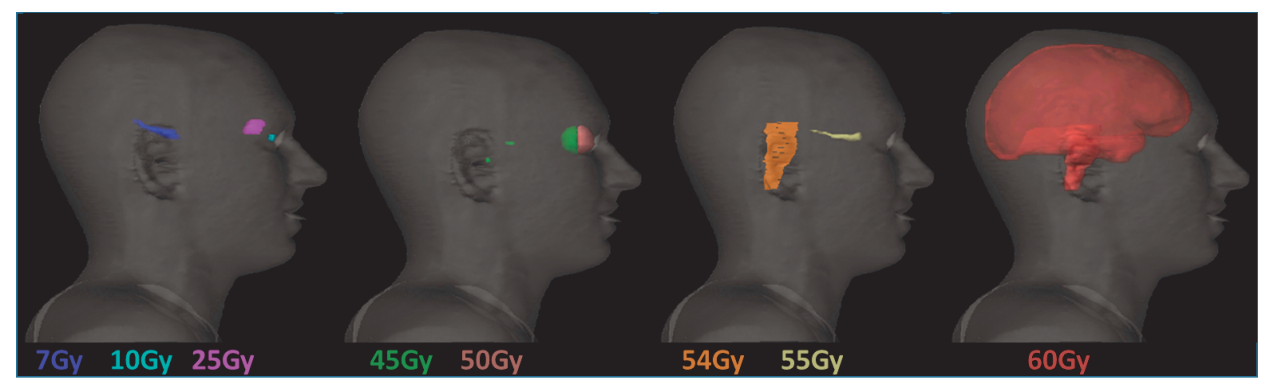

Figure 6.1 A 3D representation of the OARs and the recommended corresponding dose constraints ${ }^{1}$ : hippocampus (purple), lenses (light blue), lacrima gland (magenta), pituitary (green), cochlea (green), cornea (pink), brainstem interior (orange), chiasm (yellow), optic nerve (yellow), brainstem surface (red), brian (red). All doses are given as maximum dose to $0.03 \mathrm{cc}$ of the OAR volume $\left(D_{0.03 c c}\right.$ ), except for the dose to hippocampus, which is the $D_{40 \%}$, and the pituitary gland and cochlea, which are mean doses $\left(D_{\text {mean }}\right)$.

\section{Lacrimal gland}

The lacrimal gland system includes the main lacrimal gland, accessory lacrimal glands and the lacrimal duct system. This system is crucial for the production of tears, however, other structures, such as Meibomian glands or the conjunctival goblet cells also contribute to the production of an adequate tear film. Radiation injury to any of these structures might result in xerophthalmia or the so-called dry eye syndrome (DES) and the exact contribution of the individual components is difficult to establish ${ }^{8-10}$. DES typically develops between 1 month and 3 years after irradiation, depending on the total dose and fractionation ${ }^{9,11}$.

In the common terminology criteria for adverse events (CTCAE) version 4.0 three grades of xerophthalmia are identified ranging from mild symptoms up to a decrease in visual acuity (<20/40); limiting self-care activities of daily life (ADL) $)^{12}$. DES can lead to damage of the conjunctival and corneal epithelium (keratoconjunctivitis sicca), which causes pain, foreign body sensation, photophobia, corneal ulceration, and even perforation ${ }^{13}$.

Several retrospective series have demonstrated that the risk of atrophy and fibrosis of the lacrimal gland increases sharply with the delivered dose (Supplementary Table S6.1 $)^{9,11,14-16}$. Although the exact clinical endpoints in these series are not always clearly defined, they agree on a sigmoidal dose-response curve for DES with a negligible risk at 
absolute maximum doses $\left(D_{\max }\right)<30 \mathrm{~Gy}$, with a steeply increasing risk $>40$ Gy and a $100 \%$ rate of severe dry eye with $D_{\max }>57-60 \mathrm{~Gy}^{17,18}$.

The EPTN consensus group therefore proposes that if possible, the mean dose ( $\left.D_{\text {mean }}\right)$ to the lacrimal gland should not exceed 25 Gy for a risk for DES (> grade 1 ) less than $5 \%$. No data was found on an $\alpha / \beta$ ratio for the lacrimal gland and late dry eye syndrome, therefore we suggest to assume an $\alpha / \beta$ ratio of 3 Gy for late toxicity similar to that of the parotid gland ${ }^{19}$.

\section{Cornea}

The cornea's main functions are refraction of the light and protection, and even slight alterations of its shape can result in decreased visual acuity. Corneal complications may arise secondary to the loss of the tear film (keratitis sicca) or resulting from direct injury to the corneal surface epithelium and the deeper layers of the cornea. Direct radiation induced changes originate from the disruption of the mitotic activity in these layers and do not arise from the avascular cornea.

In CTCAE v4.0 keratitis is defined as a disorder characterized by an inflammation of the cornea with severity ranging from mild inflammation to perforation and complete blindness ${ }^{12}$.

Even though accurate dose-volume parameters are scarce, a dose-toxicity relationship has been described in several retrospective series ${ }^{13,18,20,21}$. In one retrospective analysis corneal complications were evaluated after orbital radiotherapy for lacrimal gland malignancies ${ }^{21}$. In this series patients were treated up to cumulative doses of 50-60 Gy to the entire orbit. All patients developed an acute radiation keratoconjunctivitis, 54\% of the patients had chronic corneal epithelial defects and $13 \%$ developed a corneal perforation. These perforations generally occurred within 3 years of radiotherapy. While there are several limitations to this analysis, it confirms that high dose radiotherapy can have serious consequences on the ocular surface (see Supplementary Table S6.2). We therefore propose $D_{0.03 c c}$ to the cornea not to exceed $50 \mathrm{~Gy}$ if the orbit is not part of the target volume. Again, we propose an $\alpha / \beta$ ratio of $3 \mathrm{~Gy}$ for late toxicity in absence of solid data.

\section{Retina}

The retina is the third and inner coating of the eye and is essential in visual perception. In embryogenesis both the retina and the optic nerve originate from the diencephalon and should therefore be considered as part of the central nervous system.

Retinopathy is characterized by slowly progressive microangiopathic decompensation with a focal loss of capillary endothelial cells and pericytes ${ }^{18}$. Clinically, radiation 
retinopathy includes microaneurysms, cotton wool pots, capillary dilation, telangiectasia and capillary closure, all histopathologically resembling diabetic retinopathy ${ }^{22,23}$. The latency period is typically between 6 months and 3 years, although longer periods have been described ${ }^{18,24-26}$. The CTCAE v4.0 defines retinopathy using 4 grades ranging from asymptomatic up to grade 4 blindness (20/200 or worse) in the affected eye $\mathrm{e}^{12}$. The pathogenesis of radiation induced retinopathy is dependent on the total dose, the fraction size, number of fractions, concurrent chemotherapy and coexisting morbidity, e.g., diabetes, hypertension ${ }^{23,27-29}$. A selected number of studies reported on the dose-toxicity relationship for retinopathy and are depicted in Supplementary Table S6.3 ${ }^{13,26,30,31}$.

The risk of retinopathy increases steeply with $D_{\max }$ exceeding 45-50Gy in 5 weeks. Emami et al. $^{3}$ estimated the $5 \%$ severe complication rate in 5 years (TD5/5) of the retina, i.e., visual loss, to be $45 \mathrm{~Gy}$ and the $50 \%$ severe complication rate at 5 years (TD50/5) to be $65 \mathrm{~Gy}$.

We therefore propose the $D_{0.03 c c}$ to the retina to be kept below $45 \mathrm{~Gy}$. Again, we propose an $\alpha / \beta$ ratio of $3 \mathrm{~Gy}$ for late toxicity in absence of solid data ${ }^{19}$.

\section{Lens of the eye}

The lens is a biconvex structure in the eye that helps to refract light. Any stimulus causing posterior migration and proliferation of the lens epithelial cells reduces the lens clarity, causing a cataract, and often results in some degree of visual loss ${ }^{32}$. The CTCAE v4.0 distinguishes 4 grades of cataract based on visual acuity ranging from asymptomatic (grade 1 ) to complete blindness (20/200 or worse) in the affected eye (grade 4$)^{12}$.

Irradiating the lens can lead to cataract formation. The initial insult consists of damage to the germinative zone of the lens epithelium, which leads to extensive cell death, compensatory mitosis, and the generation of the so-called 'Wedl' cells'18,27,32-35. The severity and delay until onset of radiation-induced cataracts is dose-dependent, however, the accurate threshold is poorly understood. Several retrospective studies have investigated the occurrence of cataract after irradiation ${ }^{36,37}$ (Supplementary Table S6.4).

While Emami et al. ${ }^{3}$ estimated the TD5/5 of the lens to be $10 \mathrm{~Gy}$ and the TD50/5 to be $18 \mathrm{~Gy}$, other series have demonstrated that even lower doses can result in the occurrence of cataract ${ }^{38,39}$. Recently, the International Commission on Radiological Protection (ICRP) defined 0.5 Gy as the new threshold dose for lens opacities, which is based on the data from population based studies in diagnostic imaging and occupational exposure $\mathrm{e}^{40,41}$. 
Based on these data, the EPTN consensus panel suggests the dose to the lens to be kept as low as reasonably achievable (ALARA) and should not surpass $D_{0.03 c c}$ of 10 Gy. Conversely, as replacement of a damaged lens is a relatively harmless procedure nowadays, target volume coverage should not be compromised in an attempt to spare the lenses. Since the limited data on an $\alpha / \beta$ ratio for the lens suggests values of 0.76-1.2 Gy, we propose to use an $\alpha / \beta$ ratio of $1 \mathrm{~Gy}$ for late toxicity ${ }^{19,42,43}$.

\section{Optic Nerve}

First described in 1956, radiation induced optic neuropathy (RION) is a rare yet disabling condition with a potentially devastating impact on the vision of the affected ey $\mathrm{e}^{44}$. The pathogenesis of RION is not fully understood, but it is often considered to be delayed radionecrosis in the CNS and thus the effect of radiation on the optic nerve appears to be both vascular and neuropathic in nature $23,32,45,46$. It usually presents with painless, rapid visual loss and can occur between 3 months and 8 years after treatment, with a peak between 1-1.5 years ${ }^{45,47}$. It is graded according to the CTCAE v4.0 as grade 1 being asymptomatic, grade 2 limiting vision of the affected eye (20/40 or better), grade 3 limiting vision in the affected eye (worse than 20/40) but better than 20/200 or grade 4 , blindness which is $20 / 200$ or worse in the affected eye ${ }^{12}$.

Complication data for RION have been reported for photons and protons, and following irradiation for several indications. A selected group of studies is depicted in Supplementary Table S6.5 23,48-54. Emami et al. ${ }^{3}$ suggested a TD5/5 of 50 Gy and a TD50/5 of 65 Gy. However, in the QUANTEC analysis this was deemed inaccurate after review of the literature concluding that the incidence of RION was unusual for a $D_{\max }<55$ Gy using conventional fractionation ${ }^{5}$. The incidence of RION increased between $55-60 \mathrm{~Gy}(3-7 \%)$ and was substantial (>7-20\%) for $D_{\max }>60 \mathrm{~Gy}$, although it should be noted that in some studies even at these high doses no clinically significant RION was observed. For particles most investigators also confirmed that the incidence of RION was low for a $D_{\max }<54$ Gy $(R B E)$.

Within the EPTN group we therefore support the use of $D_{0.03} \leq 55$ Gy for the optic nerve and suggest to use an $\alpha / \beta$ ratio of $2 \mathrm{~Gy}$ for late toxicity ${ }^{19}$.

\section{Optic chiasm}

In the optic chiasm, the optic nerve fibers from the nasal sides of each retina cross to the opposite side of the brain. Toxicity of the optic chiasm is graded similarly as in RION. However, instead of unilateral visual loss, it typically presents as a bitemporal hemianopsia or even total blindness. As pathophysiology is similar to RION, the same principles apply, and we suggest to use the same constraint, i.e., $D_{0.03 c c} \leq 55$ Gy and $\alpha / \beta$ 
ratio of $2 \mathrm{~Gy}^{19}$. Specific caution should be taken in patients, in whom the optic chiasm has been manipulated, e.g., during neurosurgery.

\section{Inner Ear}

The inner ear, also called labyrinth of the ear, is that part of the ear that contains the organs responsible for hearing (cochlea) and balance (vestibule and semi-circular canal). The bony labyrinth is divided into three sections: the vestibule, the semi-circular canals and the cochlea. Each section of the bony labyrinth contains perilymph and a part of the membranous labyrinth. The vestibule contains the utriculus and sacculus, the semi-circular canals contain a semi-circular duct, and the cochlea contains the cochlear duct.

\section{Cochlea}

Sensorineural hearing loss (SNHL) is the most important radiotherapy-induced complication of the inner ear, with up to $44 \%$ of patients reporting hearing loss after radiotherapy when one of the radiation beams passes the inner ear ${ }^{55,56}$. Consistently throughout the literature, the high frequencies appear to be more affected than lower frequencies, and this is dose-dependent ${ }^{56-60}$.

Hearing loss can be graded according to the CTCAE v4.0 $0^{12}$. While early hearing loss during conventionally fractionated radiotherapy is usually transient and commonly due to serous otitis media, true SNHL classically occurs with a latency period of 1.5-5 years after radiotherapy and is irreversible $55,57,61,62$. Histopathologically it results from loss of cochlear primary sensory cells and/or damage to the spiral ganglion or cochlear nerve ${ }^{63}$.

The relationship between the dose to the cochlea and SNHL has been extensively investigated. Emami et al. $^{3}$ identified a TD 5/5 of 60 Gy and TD 50/5 of 70 Gy for sensorineural or vestibular damage. However, based on more recent dose volume data the QUANTEC consensus paper suggested the $D_{\text {mean }}$ to the cochlea $\leq 45 \mathrm{~Gy}$ or even more conservatively $\leq 35 \mathrm{~Gy}^{57-60,64,65}$.

The recent publication by De Marzi et al. ${ }^{66}$, who investigated 140 patients treated with photon and proton therapy for base of skull tumours, reported on a dose-response model for the inner ear. After qualitative correlation of $D_{\text {mean }}$ with auditory toxicity (scored as grade 1-2 hearing loss, based on CTCAE v4.0), no significant cut-off value could be determined. Considering the size of the organ, they calculated the generalized equivalent uniform dose and found it to be a predictive factor for late complications. For the cochlea and inner ear, a tolerance uniform dose delivered to the whole organ for $50 \%$ complication rate (TD 50) of $56 \mathrm{~Gy}(95 \% \mathrm{Cl} 53.6-58.5)$ and $53.6 \mathrm{~Gy}(95 \% \mathrm{Cl}$ 
51.8-55.4 Gy) was reported with slope of the response curve at TD50 ( $\gamma 50)$ of 2.8 for both and an a-value of 1.2 and 0.1 , respectively. These values are in the same range as the QUANTEC data.

The EPTN consensus panel proposes the $D_{\text {mean }}$ to the cochlea to be kept to $\leq 45$ Gy. Since, there is no clear threshold dose for hearing loss after radiotherapy, the ALARA principle applies. Again, we propose an $\alpha / \beta$ ratio of $3 \mathrm{~Gy}$ for late toxicity in absence of solid data.

Besides SNHL, tinnitus is also a potential side effect from ionizing radiotherapy. CTCAE v4.0 defines tinnitus as a disorder characterized by a perception of noise or ringing in the ears, and has 3 grades, based on the impact of the tinnitus on the activities of daily life ${ }^{12}$. Limited data are available on the effect of dose on the occurrence of tinnitus and it is probably under-reported. As a result, there is no QUANTEC guideline for the cochlea to avoid tinnitus. Lee et $a .^{67}$ investigated the incidence of tinnitus after intensity modulated radiation therapy (IMRT) for head and neck cancer patients and noticed that $11.6 \%$ of developed grade $>2$ tinnitus, consistent with other reports in the literature ${ }^{68,69}$. Based on a logistic and Lyman-Kutcher NTCP model derived from their results, $D_{\text {mean }}$ to the cochlea should be kept $<32 \mathrm{~Gy}$ in order to keep the incidence of grade $>2$ tinnitus $<20 \%$ using IMRT ${ }^{67}$. External validation of this model is thus far lacking. In the absence of data, we suggest to use a traditional $\alpha / \beta$ ratio of 3 Gy for late toxicity ${ }^{19}$.

\section{Vestibulum and semi-circular canal}

Vestibular toxicity can be graded according to the CTCAE v4.0 as vertigo or more generally as a vestibular disorder ${ }^{12}$, even though occasionally acute nausea following radiotherapy is reported instead.

There is very little data concerning vestibular toxicity related to radiotherapy. Gabriele et $a l^{70}$ investigated the vestibular function in 25 head and neck cancer patients. Eleven of these patients showed vestibular abnormalities on electronystagmography, but only three reported vertigo. More recently Lee et $a .^{71}$ analysed 49 consecutive nasopharyngeal carcinoma patients treated with radiotherapy alone, of whom six reported nausea and no patient dizziness or vertigo. Using multivariate analysis, the authors identified a correlation between the volume of the vestibules receiving $40 \mathrm{~Gy}$ $\left(\mathrm{V}_{40 \mathrm{~Gy}}\right)$ and incidence of nausea. Again, external validation is awaited. Prospective collection of dose-volume data and accurate toxicity scoring is mandatory to identify dose-volume parameters in the nearby future. As such, EPTN cannot recommend any dose-constraint threshold for this OAR. 


\section{Pituitary gland/hypothalamus}

The pituitary gland is an endocrine gland essential for the regulation of many physiological processes including growth, thyroid gland function, reproduction, and lactation. It is closely linked to the hypothalamus through the pituitary stalk. Dysfunction of this hypothalamic-pituitary axis is a common problem after radiotherapy of both brain and head and neck tumours and is associated with significant morbidity and even mortality ${ }^{72-76}$. Adequate management of radiation induced hypopituitarism is essential to optimize outcomes and improve quality of life in these patients ${ }^{77}$. The CTCAE v4.0 identifies several endocrine disorders, which may be associated with hypopituitarism, although clinically it can present with a large variety of non-specific symptoms and should always be in the differential diagnosis in the follow-up of patients treated with radiotherapy for head and neck or brain tumours ${ }^{12}$. Despite its high incidence, little information on the correlation between dose and dysfunction of the hypothalamic pituitary axis is available ${ }^{74,78}$. Relevant studies are depicted in Supplementary Table S6.6 $6^{66,76,79,80}$.

In children, Merchant et al. ${ }^{81}$ described the decline in growth hormone (GH) levels after cranial radiotherapy by an exponential equation dependent on the radiation dose to the hypothalamus and the follow-up time interval, which was confirmed by Agha et $a .^{76}$ in an adult population. GH deficiency may occur after low doses ( $D_{\text {mean }}<40 \mathrm{~Gy}$ ) especially in patients whose hypothalamopituitary axis (HPA) was impaired by the presence of a tumour and/or previous surgery ${ }^{78,82,83}$. Deficiency of all anterior-pituitary hormones occurs mainly after high dose irradiation ( $D_{\text {mean }}>60 \mathrm{~Gy}$ ) in nasopharyngeal carcinoma or base of skull tumour patients (see Supplementary Table S6.6) ) $^{79,80,84}$. There is a steep increase in the incidence of endocrinopathy at a $D_{\text {mean }}$ or minimum dose $\left(D_{\min }\right)$ of $40-50 \mathrm{~Gy}$. Only one study attempted to model the NTCP for the pituitary gland using the equivalent uniform dose and found a TD50 of approx. $60.5 \mathrm{~Gy}(95 \% \mathrm{Cl}$ : 59.1-62 $\mathrm{Gy})^{66}$.

Some preclinical studies suggest there may be a differential radiosensitivity between the hypothalamus and the pituitary gland ${ }^{85}$. One study by Pai et al. ${ }^{80}$ found that doses below $20 \mathrm{~Gy}$ (RBE) to the hypothalamus were associated with endocrinopathies, while this association was only found for a $D_{\min }$ above $50 \mathrm{~Gy}(\mathrm{RBE})$ for the pituitary gland. Noteworthy, target volumes of most patients included in this analysis were located in the clivus and strict dose constraints were imposed on the optic chiasm resulting in a steep dose gradient between pituitary gland and hypothalamus. Larger dose variation and patient populations will be necessary to distinguish the individual contribution of each of these structures to HPA dysfunction. Until further data is available we propose to use the same dose constraint to both these structures. 
The EPTN consensus panel proposes a $D_{\text {mean }}<45$ Gy to the pituitary gland in the prevention of panhypopituitarism. Of course, if clinical context demands, higher doses may be justified. However, even at low doses deficiency of one of the hormonal axes might occur and a rigid endocrinological follow-up needs to be put in place, as adequate hormone replacement therapy is available and needs to be prescribed. Specific care is to be taken in patients treated for pituitary tumours or after surgery to this area, in which cases, lower tolerance doses should be employed ${ }^{77,78}$. There is currently no valuable data on the tolerance doses of the hypothalamus. As such, EPTN cannot recommend any dose-constraint threshold for the hypothalamus at this stage. Prospective and standardized reporting of dose and toxicity might help us to overcome this. In addition, there is no clear data on the $\alpha / \beta$ ratio of the pituitary gland or hypothalamus for the endpoint hypopituitarism, therefore we suggest to use an $\alpha / \beta$ ratio of 2 Gy for late toxicity.

\section{Brain}

Damage to the CNS is of considerable concern in the radiation treatment of brain tumours. However, evaluating cerebral toxicity is extremely difficult as we only begin to understand the intricate interplay between the different substructures in physiological conditions, let alone in pathological conditions. In this review two main long-term adverse effects will be evaluated mainly radionecrosis and neurocognition.

\section{Brain}

Despite its complexity, dose constraints to the brain and cerebrum are uniformly applied to the entire cerebral parenchyma without distinction between cortex, white matter and nuclei. Concerning radionecrosis, Emami et al. ${ }^{3}$ reported a TD5/5 of $60 \mathrm{~Gy}$, $50 \mathrm{~Gy}$ and $45 \mathrm{~Gy}$ and a TD50/5 of $75 \mathrm{~Gy}, 65 \mathrm{~Gy}$ and $60 \mathrm{~Gy}$ if 1/3, 2/3 or the entire brain, respectively, was irradiated up to that dose. These values appear to be too conservative in the 3D era, as the QUANTEC project found a dose response relationship in the brain: the incidence of radionecrosis increases from $3 \%$ with a $D_{\max }<60 \mathrm{~Gy}$, to $5 \%$ at $D_{\max }=72 \mathrm{~Gy}$, and to $10 \%$ when $D_{\max }=90 \mathrm{~Gy}$, using an $\alpha / \beta=3 \mathrm{~Gy}^{4,86}$. Following the QUANTEC data, several papers reported on the dose-volume relationship for temporal lobe necrosis using both photons and protons ${ }^{87-90}$. They all highlight the importance of the volume receiving a certain dose in the occurrence of brain necrosis. The results are depicted in Supplementary Table S6.7.

Based on these data, the EPTN consensus panel proposes to $\mathrm{V}_{60 G y}<3 \mathrm{cc}$ in EQD2. The $\alpha / \beta$ ratio for brain tissue is $2 \mathrm{~Gy}$ for radionecrosis ${ }^{19}$. 
Aside from radionecrosis, radiation induced white matter damage can also cause serious neurocognitive disturbances ${ }^{91}$. However, to our knowledge there is no clear dose-volume data available allowing us to selectively spare a specific part of the supratentorial brain. As such we cannot recommend any dose constraint threshold for brain and neurocognition, and thus the ALARA principle applies.

\section{Brainstem}

The brainstem consists of the medulla oblongata, pons and midbrain. It plays a crucial role as a relay between the body, the cerebellum and cerebrum, it gives rise to nine functions. Damage to the brainstem is therefore a severe and potentially lethal complication and can present as a wide spectrum of clinical features depending on the location and the extent of the damage ${ }^{92}$. This of course has important implications for the dose constraints; unlike for several other OARs, no long-term toxicity should occur at the level of the brain stem. There are several recommendations based on the available literature and there seems to be a clear distinction between the dose constraints used for photons and protons ${ }^{6,92}$. An overview of some selected reports on planning constraints and toxicity are depicted in Supplementary Table S6.8 $8^{52,93-101}$.

Historically, Emami et al. ${ }^{3}$ defined the TD5/5 for necrosis of the brainstem as $50 \mathrm{~Gy}$, $53 \mathrm{~Gy}$ and $60 \mathrm{~Gy}$ to the entire, 2/3 and $1 / 3$ of the volume of the brainstem, respectively, and the TD50/5 of the entire brainstem was estimated at $65 \mathrm{~Gy}^{102}$. However, these values appear to be overly conservative considering the data available from recent retrospective analysis. It appears that the entire brainstem may be treated to $54 \mathrm{~Gy}$ using conventional fractionation with limited risk of severe or permanent neurological effects, while small volumes of the brainstem may even be irradiated to $D_{\max }=59 \mathrm{~Gy}$. The risk appears to increase markedly for $D_{\max }>64 G y^{6,103}$.

The consensus panel therefore suggests $D_{0.03 c c}<54$ Gy in EQD2, in particular to the interior to the brainstem. Whenever institutions opt to use higher doses, we propose that $D_{0.03 c c}$ of the brainstem surface should be kept $<60$ Gy EQD2, which correlates with the absolute dose of 64Gy RBE used in the proton literature for base of skull ${ }^{97-101}$. For both, the brain and brainstem, we assume an $\alpha / \beta$ ratio of 2 Gy for late CNS toxicity ${ }^{19}$.

\section{Hippocampus}

One of the most elusive long-term toxicities related to radiotherapy of the brain is neurocognitive decline and memory impairment. It has become exceedingly important in the debate surrounding the use of more complex and expensive radiotherapy techniques, even though the exact pathophysiology is complex and poorly understood ${ }^{104-106}$. In the time of the QUANTEC project there was insufficient evidence 
to support the claim that partial brain radiotherapy in 2 Gy fractions causes neurocognitive decline ${ }^{7}$. This was partly due to insufficient outcome measurements as well as the lack of detailed brain dose-volume data. However, over the last decade there is a growing insight in the mechanisms behind neurocognitive disability after radiotherapy, particularly in respect to the hippocampi, which are instrumental in learning, memory and neurogenesis ${ }^{104,107}$. The seminal article by Gondi et al. ${ }^{108}$ compared a control group with a historical group of patients treated for benign tumours or low grade gliomas. They found that if the $\mathrm{D}_{40 \%}$ of the bilateral hippocampi exceeded 7.3 Gy this was associated with a decrease in the WMS-WL delayed recall test at 18 months. Many studies are currently underway, investigating hippocampal avoidance in several clinical settings, however, we are still awaiting results. Imaging studies have revealed that doses exceeding $40 \mathrm{~Gy}$ resulted in a significant atrophy of the hippocampus (see Supplementary Table S6.8) ${ }^{109}$.

Based on these data it is somewhat preliminary to propose dose constraints to the hippocampus. If possible, the dose to the hippocampi should be kept ALARA and preferably the $D_{40 \%}$ of both hippocampi combined should be kept below $7.3 \mathrm{~Gy}$. Again, an $\alpha / \beta$ ratio of 2 Gy for late CNS toxicity.

\section{Cerebellum}

Classically, the cerebellum is known for its role in the regulation and coordination of movement posture and balance. Radionecrosis could thus have an important influence on these factors. For this outcome there is currently no data suggesting a different radiosensitivity of the cerebellar cortex or white matter, and thus the same constraints as those used in the brain are proposed.

Interestingly there is more and more evidence that the cerebellum is also involved in cognitive functions ${ }^{110}$. In paediatric patients with infratentorial ependymoma one report found a correlation between the infratentorial radiation dose and neurocognitive decline ${ }^{111}$. However, in adults this evidence is lacking and so to date no clear dose constraints can be defined for the anterior and posterior cerebellum.

Skin

Radiation to the scalp can give rise to several toxicities. Since the skin itself is rarely part of the target volume in primary brain tumours, temporary and permanent alopecia is the most important late toxicity after radiotherapy. Temporary alopecia can occur after very low doses, while permanent alopecia requires relatively higher doses to the skin $^{112,113}$. Lawenda et al. ${ }^{114}$ performed an depth dose response analysis on permanent alopecia at $>12$ months in patients treated with photons for a primary CNS tumour. 
After multivariate analysis, only the follicle dose was significantly correlated to permanent alopecia. In the dose-response relationship the $\mathrm{TD}_{50}$ was estimated at $43 \mathrm{~Gy}$ EQD2 (95\%Cl 33-52) with a $y 50$ slope of 0.9 (95\% Cl 0.3-1.4). Using this dose-response relationship, a follicle dose of $25 \mathrm{~Gy}$ is associated with $<20 \%$ risk of permanent alopecia grade $\geq 3$. In order to avoid this side effect a reduction of the dose to the hair follicles should be attempted.

The EPTN therefore suggests the $D_{0.03 c c}$ of the skin should be kept $<25$ Gy to avoid permanent focal alopecia and consequently the $V_{25 G y}$ to the skin should be kept ALARA. The suggested $\alpha / \beta$ ratio for the skin is $2 \mathrm{~Gy}^{19,114}$.

\section{Discussion}

Despite the increasing number of patients treated with radiotherapy for brain tumours, scarce precise information is available on the relationship between dose and toxicity of the central nervous system. In the past several efforts have already been undertaken to try and summarize the available evidence on the tolerance of normal tissues ${ }^{3,4,102}$. However, several relevant OARs, such as the lacrimal gland, the cornea, the vestibulum and semi-circular canals, the HPA, hippocampus, cerebellum and skin were not discussed in these papers. In addition, the increasing availability of highly conformal photon and proton therapy, the widespread use of image-guided and adaptive radiotherapy enable the radiotherapy community to deliver high doses to the target volume and selectively spare certain organs at risk. While dosimetrically these techniques might produce 'better' treatment plans, they do not always translate into clinical reality. In order to justify the use of these expensive treatments and make an accurate estimation of the benefit of one technique over the other, an objective estimation of the normal tissue complication rate is crucial ${ }^{115,116}$. Such a model-based approach allows us to compare different treatment strategies and select patients who will most likely benefit from a certain technique based on the difference in NTCP models between two techniques. These NTCP models need to incorporate both dosimetric and clinical factors and provide us with objective data on the superiority of one technique over the other ${ }^{4,102,117}$. However, the construction of these models requires an large amount of uniformly scored patient data. Consequently, for all OAR and toxicities described in the manuscript, such a multifactorial NTCP model is not available.

Therefore, one of the key goals of the EPTN is to try and set-up a framework for international cooperation within the radiotherapy community, which allows the introduction of a uniform, consensus-based means for data collection. In a first 
consensus paper, relevant OARs in neuro-oncology were selected and delineation guidelines were given ${ }^{1}$. This manuscript aimed to review the available evidence on the dose-toxicity relationship for the previously defined OAR. While we succeeded in producing a consensus table on dose constraints (see Table 6.1), there are several shortcomings of the data presented here.

First, as is clear from this review, the vast majority of dose constraints rely on the reports from retrospective, single centre studies. Furthermore, in most of the cases no accurate dose-volume analysis could be done as the majority of patients within each series is treated for a variety of primary tumours with a variety of doses and fractionation schedules, using old radiotherapy techniques, and without uniform contouring of the OARs. We can therefore only estimate the dose delivered to a certain OAR. Also, in the majority of cases absolute doses are reported, with little to no information on the exact fractionation, making it impossible to recalculate the doses to EQD2. For the consensus table we aimed to define all $D_{\max }$ constraints in EQD2, using the linear quadratic formula, this allows us to recalculate the dose depending on the

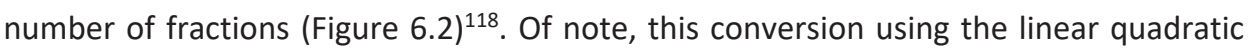
formula does not apply to $D_{\text {mean }}$ dose constraints. Instead, the mean dose to the OAR and its standard deviation is required for this conversion, and therefore, we cannot provide these technique-dependent values ${ }^{119,120}$. In keeping with the ICRU 83 and 91 reports we avoided using the maximum absorbed dose at a single calculation point $\left(D_{\max }\right)$ for the constraint table ${ }^{121,122}$. However, for larger OARs such as the brain and brainstem, the classical $D_{2} \%$ might result in a relatively large volume exceeding the tolerance dose. We therefore opted to use the $D_{0.03 c c}$ as this approximates the $D_{\max }$ used in the majority of the literature, since this depends less on the treatment planning system and scanner parameters used, and thus provides a more realistic calculation of the delivered dose. Only dose parameters related with specific toxicities were included in the consensus table, however, for planning purposes it is obvious that more than one dose-volume parameter should be included in the optimization process (as objectives or constraints) to ensure the optimal plan is generated.

Second, when setting dose constraints we assume a dose volume effect for the structures, and set a threshold below which the odds of a certain toxicity are reasonable. The outcome of this exercise depends highly on the severity of the toxicity and at what cost the dose is pushed below a certain threshold. Conversely, it is obvious that RT related toxicity is a more complex and multifactorial process where genetic disposition, co-morbidities, dosimetric and clinical factors all play an important role in explaining why some patients experience excess toxicity at low doses and others can be treated up to high doses without any toxicity ${ }^{117}$. This interplay is impossible to grasp in a single parameter and, again, requires more complex models, which incorporate 
clinical, genetic, and dosimetric factors into a multifactorial NTCP model which allows for an individual patient-based risk assessment.

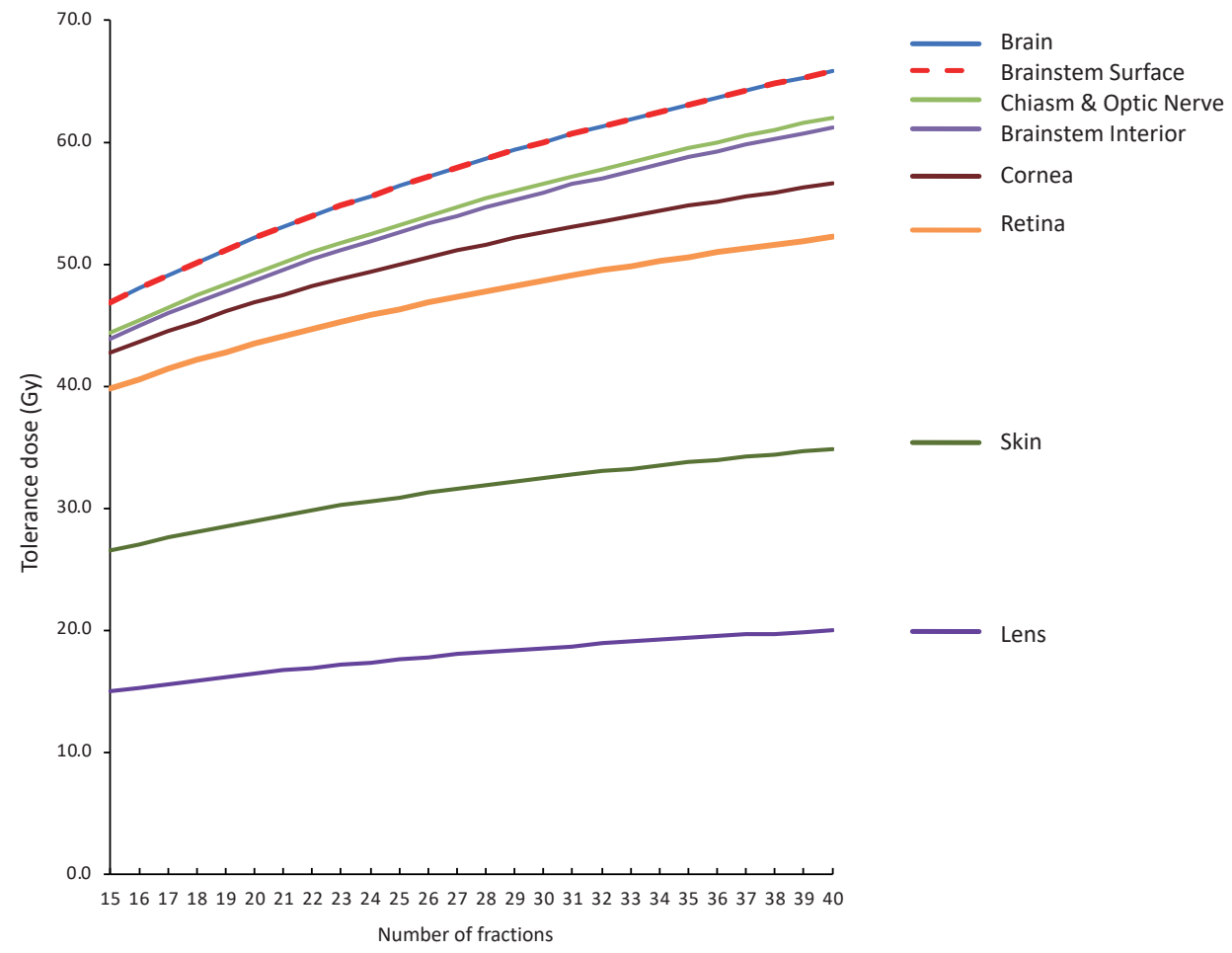

Figure 6.2 Tolerance dose versus total number of fractions. The Tolerance dose is calculated using the EPTN consensus dose constraints and $\mathrm{a} / \mathrm{b}$ versus the total number of fractions. On the $\mathrm{X}$-axis the total number of fractions is given and on the $Y$-axis the corresponding physical tolerance dose (Gy); for all OAR the $\mathrm{D}_{0.03 \mathrm{cc}}$ (= dose to $0.03 \mathrm{cc}$ of the organ/structure) except for the brain ( $D_{3 c c}=$ dose to $3 \mathrm{cc}$ of the brain).

Third, toxicity was not uniformly scored in all series. In the review several toxicity scoring mechanisms have been used, with the RTOG/EORTC Late Effects Normal Tissue Task Force subjective, objective, management, and analytic (LENT/SOMA) score and CTCAE being the most prominently used ${ }^{12,123}$. Uniform scoring of toxicity is of utmost importance when constructing a prospective database. Throughout the manuscript, we promote the use of the CTCAE scoring system as it is widely accepted and applicable and allows for a more detailed scoring of the severity of toxicity compared to the LENT/SOMA evaluation. Aside from the physician scored toxicity, patient reported 
outcome (PRO) scoring systems should also be implemented, in the prospective data collection as several studies have demonstrated that there is severe discrepancy between patients and physician reported toxicities and that generally physicians tend to underreport the presence and severity of treatment related toxicities ${ }^{124,125}$.

Fourth, it is very important to realize that there is an interplay between different structures when looking at toxicity. For example, while DES and corneal damage are described separately, in reality they are very closely interlinked. This interplay, however, is not taken into account when proposing a single dose constraint for the lacrimal gland. The problem becomes even more complex when looking at a toxicity which is multifactorial in principle such as neurocognition ${ }^{108,110,126}$. While the hippocampus was among the first structures to be related to neurocognitive decline, and is instrumental in learning and memory, it is not the only structure responsible for good cognitive functioning. The prefrontal cortex, the cerebellum and the hypothalamus all play an important role in the higher cognitive function of the brain ${ }^{106,110,126,127}$. To what extent radiation induced damage to these structures impairs their function is far less understood. With increasing practice to spare the hippocampus, the relative importance of these or other structures will become increasingly important.

Therefore, it is important that the summary constraint table in this manuscript is not to be considered as an endpoint. As radiation techniques evolve, treatment changes and survival improves, relevant toxicities will also evolve. While some toxicities were initially of great importance and dose limiting, they can become less frequent and other toxicities gain the upper hand. An example for this is optic neuropathy. While frequently reported in older papers, there have been no relevant papers on this toxicity using conventionally fractionated radiotherapy since the QUANTEC report ${ }^{5}$. This can partly be explained by the combination of clear dose constraints with the ability to effectively reduce the dose to the optic nerve using conformal radiotherapy techniques. The true value of this manuscript lies in the fact that it provides a consensus on the dose constraints for relevant OARs in the treatment of brain tumours among experts in the field. Of note, it is only a consensus, with all the shortcomings described above. Together with the delineation guidelines by Eekers et al. ${ }^{1}$, however, it is a starting point for uniform OAR delineation and dose prescription. If we succeed in setting up a standardized follow-up with prospective scoring of toxicity it provides a basic framework within which a large number of patients are treated and followed uniformly and thus can be used to develop and validate multifactorial NTCP models.

Fifth, all constraints in Table 6.1 are reported in EQD2 unless otherwise reported. To facilitate conversion we suggested an $\alpha / \beta$ ratio based on the best available evidence found, while it should be noted that there is considerable uncertainty regarding these 
ratios $^{19,128}$. In principle, the dose constraints are useful for both photon and proton radiotherapy provided that a conversion factor of 1.1 for conventional fractionation is used to account for the difference in $\mathrm{RBE}^{129}$. However, at the very distal edge of the Bragg peak, the linear energy transfer is higher, resulting in an increased $R^{2} E^{130}$. This is of concern as the distal edge of the Bragg peak is often very close or even overlapping with the dose-limiting OAR and might result in unexpectedly high toxicity or abnormal imaging changes ${ }^{131-133}$. Therefore, close observation of all these patients remains crucial.

Finally, the set of dose constraints aims to provide assistance to the physician and physicist/dosimetrist in the difficult task of coming up with the optimal plan for each patient, balancing out tumour control and potential toxicity with regard to the age, comorbidities and life expectancy of the patient. From this review it should be obvious that they are not absolute values and the risks and benefits of each treatment should be thoroughly discussed with the patient. 


\section{References}

1. Eekers DBP, in 't Ven L, Roelofs E, Postma A, Alapetite C, Burnet N, et al. The EPTN consensus-based atlas for CT- and MR-based contouring in Neuro-Oncology. Radiother Oncol 2018;128(1):37-43.

2. Eekers DBP, in 't Ven L, Roelofs E, Postma A, Troost EGC. EPTN International Neurological Contouring Atlas. CancerData 2017. doi:10.17195/candat.2017.08.1.

3. Emami B, Lyman J, Brown A, Coia L, Goitein M, Munzenrider JE, et al. Tolerance of normal tissue to therapeutic irradiation. Int J Radiat Oncol Biol Phys 1991;21:109-22.

4. Marks LB, Yorke ED, Jackson A, Ten Haken RK, Constine LS, Eisbruch A, et al. Use of normal tissue complication probability models in the clinic. Int J Radiat Oncol Biol Phys 2010;76:S10-9.

5. Mayo C, Martel MK, Marks LB, Flickinger J, Nam J, Kirkpatrick J. Radiation Dose-Volume Effects of Optic Nerves and Chiasm. Int J Radiat Oncol 2010;76:S28-35.

6. Mayo C, Yorke E, Merchant TE. Radiation associated brainstem injury. Int J Radiat Oncol Biol Phys 2010;76:S36-41.

7. Lawrence YR, Li XA, el Naqa I, Hahn CA, Marks LB, Merchant TE, et al. Radiation dose-volume effects in the brain. Int J Radiat Oncol Biol Phys 2010;76:S20-7.

8. Woo YJ, Ko J, Ji YW, Kim T, Yoon JS. Meibomian Gland Dysfunction Associated With Periocular Radiotherapy. Cornea 2017;36:1486-91.

9. Parsons JT, Bova FJ, Fitzgerald CR, Mendenhall WM, Million RR. Severe dry-eye syndrome following external beam irradiation. Int J Radiat Oncol Biol Phys 1994;30:775-80.

10. Thariat J, Maschi C, Lanteri S, Peyrichon ML, Baillif S, Herault J, et al. Dry Eye Syndrome After Proton Therapy of Ocular Melanomas. Int J Radiat Oncol Biol Phys 2017;98:142-51.

11. Bhandare N, Moiseenko V, Song WY, Morris CG, Tariq Bhatti M, Mendenhall WM. Severe dry eye syndrome after radiotherpay for head-and-neck tumors. Radiat Oncol Biol 2012;82:1501-8.

12. Cancer Institute N. Common Terminology Criteria for Adverse Events (CTCAE) Common Terminology Criteria for Adverse Events v4.0 (CTCAE) 2009.

13. Nakissa N, Rubin P, Strohl R, Keys H. Ocular and orbital complications following radiation therapy of paranasal sinus malignancies and review of literature. Cancer 1983;51:980-6.

14. Bessell EM, Henk JM, Whitelocke RAF, Wright JE. Ocular morbidity after radiotherapy of orbital and conjunctival lymphoma. Eye 1987;1:90-6.

15. Letschert JG, González González D, Oskam J, Koornneef L, van Dijk JD, Boukes R, et al. Results of radiotherapy in patients with stage I orbital non-Hodgkin's lymphoma. Radiother Oncol 1991;22:36-44.

16. Batth SS, Sreeraman R, Dienes E, Beckett LA, Daly ME, Cui J, et al. Clinical-dosimetric relationship between lacrimal gland dose and ocular toxicity after intensity-modulated radiotherapy for sinonasal tumours. Br J Radiol 2013;86:20130459.

17. Parsons JT, Bova FJ, Mendenhall WM, Million RR, Fitzgerald CR. Response of the normal eye to high dose radiotherapy. Oncology (Williston Park) 1996;10:837-47-8, 851-2.

18. Jeganathan VSE, Wirth A, MacManus MP. Ocular Risks From Orbital and Periorbital Radiation Therapy: A Critical Review. Int J Radiat Oncol 2011;79:650-9.

19. Kehwar TS. Analytical approach to estimate normal tissue complication probability using best fit of normal tissue tolerance doses into the NTCP equation of the linear quadratic model. J Cancer Res Ther 2005;1:168-79.

20. Barabino S, Raghavan A, Loeffler J, Dana R. Radiotherapy-induced ocular surface disease. Cornea 2005;24:909-14.

21. Gore SK, Plowman NP, Dharmasena A, Verity DH, Rose GE. Corneal complications after orbital radiotherapy for primary epithelial malignancies of the lacrimal gland. $\mathrm{Br} J$ Ophthalmol 2017:bjophthalmol-2017-311134.

22. Archer DB, Amoaku WMK, Gardiner TA. Radiation retinopathy-Clinical, histopathological, ultrastructural and experimental correlations. Eye 1991;5:239-51.

23. Parsons JT, Bova FJ, Fitzgerald CR, Mendenhall WM, Million RR. Radiation optic neuropathy after megavoltage external-beam irradiation: analysis of time-dose factors. Int J Radiat Oncol Biol Phys 1994;30:755-63. 
24. Gupta A, Dhawahir-Scala F, Smith A, Young L, Charles S. Radiation Retinopathy: Case report and review. BMC Ophthalmol 2007;7:6.

25. Brown GC, Shields JA, Sanborn G, Augsburger JJ, Savino PJ, Schatz NJ. Radiation retinopathy. Ophthalmology 1982;89:1494-501.

26. Takeda A, Shigematsu N, Suzuki S, Fujii M, Kawata T, Kawaguchi O, et al. Late retinal complications of radiation therapy for nasal and paranasal malignancies: relationship between irradiated-dose area and severity. Int J Radiat Oncol Biol Phys 1999;44:599-605.

27. Durkin SR, Roos D, Higgs B, Casson RJ, Selva D. Ophthalmic and adnexal complications of radiotherapy. Acta Ophthalmol Scand 2007;85:240-50.

28. Viebahn M, Barricks ME, Osterloh MD. Synergism between diabetic and radiation retinopathy: case report and review. Br J Ophthalmol 1991;75:629-32.

29. Chan RC, Shukovsky L. Effects of Irradiation on the Eye. Radiology 1976;120:673-5.

30. Parsons JT, Bova FJ, Fitzgerald CR, Mendenhall WM, Million RR. Radiation retinopathy after externalbeam irradiation: analysis of time-dose factors. Int J Radiat Oncol Biol Phys 1994;30:765-73.

31. Evans JR, Sivagnanavel V, Chong V. Radiotherapy for neovascular age-related macular degeneration. In: Chong V, editor. Cochrane Database Syst. Rev., Chichester, UK: John Wiley \& Sons, Ltd; 2010, p. CD004004.

32. Gordon KB, Char DH, Sagerman RH. Late effects of radiation on the eye and ocular adnexa. Int J Radiat Oncol 1995;31:1123-39.

33. Worgul B V, Merriam GR, Szechter A, Srinivasan D. Lens epithelium and radiation cataract. I. Preliminary studies. Arch Ophthalmol (Chicago, III 1960) 1976;94:996-9.

34. Worgul B V, Merriam GR, Medvedovsky C. Cortical cataract development--an expression of primary damage to the lens epithelium. Lens Eye Toxic Res 1989;6:559-71.

35. Merriam GR, Worgul B V, Worgul B V. Experimental radiation cataract--its clinical relevance. Bull N Y Acad Med 1983;59:372-92.

36. Henk JM, Whitelocke RA, Warrington AP, Bessell EM. Radiation dose to the lens and cataract formation. Int J Radiat Oncol Biol Phys 1993;25:815-20.

37. Deeg HJJ, Flournoy N, Sullivan KM, Sheehan K, Buckner CDD, Sanders JE, et al. Cataracts after total body irradiation and marrow transplantation: a sparing effect of dose fractionation. Int J Radiat Oncol Biol Phys 1984;10:957-64.

38. Merriam GR, Focht EF. A clinical study of radiation cataracts and the relationship to dose. Am J Roentgenol Radium Ther Nucl Med 1957;77:759-85.

39. Schipper J, Tan KE, van Peperzeel HA. Treatment of retinoblastoma by precision megavoltage radiation therapy. Radiother Oncol 1985;3:117-32.

40. Kleiman NJ. Radiation cataract. Ann ICRP 2012;41:80-97.

41. Stewart FA, Akleyev AV, Hauer-Jensen M, Hendry JH, Kleiman NJ, MacVittie TJ, et al. ICRP PUBLICATION 118: ICRP Statement on Tissue Reactions and Early and Late Effects of Radiation in Normal Tissues and Organs - Threshold Doses for Tissue Reactions in a Radiation Protection Context. Ann ICRP 2012;41: 1-322.

42. Schenken LL, Hagemann RF. Time/dose relationships in experimental radiation cataractogenesis. Radiology 1975;117:193-8.

43. Hall MD, Schultheiss TE, Smith DD, Nguyen KH, Wong JYC. Dose response for radiation cataractogenesis: a meta-regression of hematopoietic stem cell transplantation regimens. Int J Radiat Oncol Biol Phys 2015;91:22-9.

44. Forrest AP, Brown DAP, Morris SR, Illingsworth CF. Pituitary radon implant for advanced cancer. Lancet (London, England) 1956;270:399-401.

45. Kline LB, Kim JY, Ceballos R. Radiation optic neuropathy. Ophthalmology 1985;92:1118-26.

46. Danesh-Meyer H V. Radiation-induced optic neuropathy. J Clin Neurosci 2008;15:95-100.

47. McClellan RL, el Gammal T, Kline LB. Early bilateral radiation-induced optic neuropathy with follow-up MRI. Neuroradiology 1995; 37:131-3.

48. Jiang GL, Tucker SL, Guttenberger R, Peters LJ, Morrison WH, Garden AS, et al. Radiation-induced injury to the visual pathway. Radiother Oncol 1994;30:17-25. 
49. Goldsmith BJ, Rosenthal SA, Wara WM, Larson DA. Optic neuropathy after irradiation of meningioma. Radiology 1992;185:71-6.

50. Martel MK, Sandler HM, Cornblath WT, Marsh LH, Hazuka MB, Roa WH, et al. Dose-volume complication analysis for visual pathway structures of patients with advanced paranasal sinus tumors. Int J Radiat Oncol Biol Phys 1997;38:273-84.

51. Flickinger JC, Lunsford LD, Singer J, Cano ER, Deutsch M. Megavoltage external beam irradiation of craniopharyngiomas: analysis of tumor control and morbidity. Int J Radiat Oncol Biol Phys 1990;19: 117-22.

52. Hoppe BS, Stegman LD, Zelefsky MJ, Rosenzweig KE, Wolden SL, Patel SG, et al. Treatment of nasal cavity and paranasal sinus cancer with modern radiotherapy techniques in the postoperative settingthe MSKCC experience. Int J Radiat Oncol 2007;67:691-702.

53. Mackley HB, Reddy CA, Lee S-Y, Harnisch GA, Mayberg MR, Hamrahian AH, et al. Intensity-modulated radiotherapy for pituitary adenomas: the preliminary report of the Cleveland Clinic experience. Int J Radiat Oncol Biol Phys 2007;67:232-9.

54. Bhandare N, Monroe AT, Morris CG, Bhatti MT, Mendenhall WM. Does altered fractionation influence the risk of radiation-induced optic neuropathy? Int J Radiat Oncol 2005;62:1070-7.

55. Bhide SA, Harrington KJ, Nutting CM. Otological toxicity after postoperative radiotherapy for parotid tumours. Clin Oncol (R Coll Radiol) 2007;19:77-82.

56. Raaijmakers E, Engelen AM. Is sensorineural hearing loss a possible side effect of nasopharyngeal and parotid irradiation? A systematic review of the literature. Radiother Oncol 2002;65:1-7.

57. Kwong DL, Wei WI, Sham JS, Ho WK, Yuen PW, Chua DT, et al. Sensorineural hearing loss in patients treated for nasopharyngeal carcinoma: a prospective study of the effect of radiation and cisplatin treatment. Int J Radiat Oncol Biol Phys 1996;36:281-9.

58. Pan CC, Eisbruch A, Lee JS, Snorrason RM, Ten Haken RK, Kileny PR. Prospective study of inner ear radiation dose and hearing loss in head-and-neck cancer patients. Int J Radiat Oncol Biol Phys 2005;61:1393-402.

59. Chen WC, Jackson A, Budnick AS, Pfister DG, Kraus DH, Hunt MA, et al. Sensorineural hearing loss in combined modality treatment of nasopharyngeal carcinoma. Cancer 2006;106:820-9.

60. Honoré HB, Bentzen SM, Møller K, Grau C. Sensori-neural hearing loss after radiotherapy for nasopharyngeal carcinoma: individualized risk estimation. Radiother Oncol 2002;65:9-16.

61. Borsanyi SJ, Blanchard CL. Ionizing Radiation and the Ear. JAMA J Am Med Assoc 1962;181:958.

62. Leach W. Irradiation of the Ear. J Laryngol Otol 1965;79:870-80.

63. Linskey ME, Johnstone PA. Radiation tolerance of normal temporal bone structures: implications for gamma knife stereotactic radiosurgery. Int J Radiat Oncol 2003;57:196-200.

64. Oh Y-T, Kim C-H, Choi J-H, Kang S-H, Chun M. Sensory neural hearing loss after concurrent cisplatin and radiation therapy for nasopharyngeal carcinoma. Radiother Oncol 2004;72:79-82.

65. Bhandare N, Jackson A, Eisbruch A, Pan CC, Flickinger JC, Antonelli P, et al. Radiation therapy and hearing loss. Int J Radiat Oncol Biol Phys 2010;76:S50-7.

66. De Marzi L, Feuvret L, Boulé T, Habrand J-L, Martin F, Calugaru V, et al. Use of gEUD for predicting ear and pituitary gland damage following proton and photon radiation therapy. $\mathrm{Br} \mathrm{J}$ Radiol 2015;88:20140413.

67. Lee T-F, Yeh S-A, Chao P-J, Chang L, Chiu C-L, Ting H-M, et al. Normal tissue complication probability modeling for cochlea constraints to avoid causing tinnitus after head-and-neck intensity-modulated radiation therapy. Radiat Oncol 2015;10:194.

68. van der Putten L, de Bree R, Plukker JT, Langendijk JA, Smits C, Burlage FR, et al. Permanent unilateral hearing loss after radiotherapy for parotid gland tumors. Head Neck 2006;28:902-8.

69. Tuan JKL, Ha TC, Ong WS, Siow TR, Tham IWK, Yap SP, et al. Late toxicities after conventional radiation therapy alone for nasopharyngeal carcinoma. Radiother Oncol 2012;104:305-11.

70. Gabriele P, Orecchia R, Magnano M, Albera R, Sannazzari GL. Vestibular apparatus disorders after external radiation therapy for head and neck cancers. Radiother Oncol 1992;25:25-30.

71. Lee VHF, Ng SCY, Leung TW, Au GKH, Kwong DLW. Dosimetric Predictors of Radiation-induced Acute Nausea and Vomiting in IMRT for Nasopharyngeal Cancer. Int J Radiat Oncol 2012;84:176-82. 
72. Tomlinson JW, Holden N, Hills RK, Wheatley K, Clayton RN, Bates AS, et al. Association between premature mortality and hypopituitarism. West Midlands Prospective Hypopituitary Study Group. Lancet (London, England) 2001;357:425-31.

73. Appelman-Dijkstra NM, Malgo F, Neelis KJ, Coremans I, Biermasz NR, Pereira AM. Pituitary dysfunction in adult patients after cranial irradiation for head and nasopharyngeal tumours. Radiother Oncol 2014;113:102-7.

74. Kyriakakis N, Lynch J, Orme SM, Gerrard G, Hatfield P, Loughrey C, et al. Pituitary dysfunction following cranial radiotherapy for adult-onset nonpituitary brain tumours. Clin Endocrinol (Oxf) 2016;84:372-9.

75. Sherlock M, Ayuk J, Tomlinson JW, Toogood AA, Aragon-Alonso A, Sheppard MC, et al. Mortality in Patients with Pituitary Disease. Endocr Rev 2010;31:301-42.

76. Agha A, Sherlock M, Brennan S, O’Connor SA, O'Sullivan E, Rogers B, et al. Hypothalamic-Pituitary Dysfunction after Irradiation of Nonpituitary Brain Tumors in Adults. J Clin Endocrinol Metab 2005;90:6355-60.

77. Fernandez A, Brada M, Zabuliene L, Karavitaki N, Wass JAH. Radiation-induced hypopituitarism. Endocr Relat Cancer 2009;16:733-72.

78. Littley MD, Shalet SM, Beardwell CG, Ahmed SR, Applegate G, Sutton ML. Hypopituitarism following external radiotherapy for pituitary tumours in adults. Q J Med 1989;70:145-60.

79. Bhandare N, Kennedy L, Malyapa RS, Morris CG, Mendenhall WM. Hypopituitarism after radiotherapy for extracranial head and neck cancers. Head Neck 2008;30:1182-92.

80. Pai HH, Thornton A, Katznelson L, Finkelstein DM, Adams JA, Fullerton BC, et al. Hypothalamic/pituitary function following high-dose conformal radiotherapy to the base of skull: demonstration of a doseeffect relationship using dose-volume histogram analysis. Int J Radiat Oncol Biol Phys 2001;49:1079-92.

81. Merchant TE, Goloubeva O, Pritchard DL, Gaber MW, Xiong X, Danish RK, et al. Radiation Dose-Volume Effects on Growth Hormone Secretion n.d.

82. Brada M, Rajan B, Traish D, Ashley S, Holmes-Sellors PJ, Nussey S, et al. The long-term efficacy of conservative surgery and radiotherapy in the control of pituitary adenomas. Clin Endocrinol (Oxf) 1993;38:571-8.

83. Langsenlehner T, Stiegler C, Quehenberger F, Feigl GC, Jakse G, Mokry M, et al. Long-Term Follow-up of Patients with Pituitary Macroadenomas after Postoperative Radiation Therapy. Strahlentherapie Und Onkol 2007;183:241-7.

84. Lam KS, Tse VK, Wang C, Yeung RT, Ho JH. Effects of cranial irradiation on hypothalamic-pituitary function--a 5-year longitudinal study in patients with nasopharyngeal carcinoma. Q J Med 1991;78: 165-76.

85. Robinson IC, Fairhall KM, Hendry JH, Shalet SM. Differential radiosensitivity of hypothalamo-pituitary function in the young adult rat. J Endocrinol 2001;169:519-26.

86. Lawrence YR, Li XA, el Naqa I, Hahn CA, Marks LB, Merchant TE, et al. Radiation Dose-Volume Effects in the Brain. Int J Radiat Oncol 2010;76:S20-7.

87. Su S-F, Huang Y, Xiao W, Huang S-M, Han F, Xie C, et al. Clinical and dosimetric characteristics of temporal lobe injury following intensity modulated radiotherapy of nasopharyngeal carcinoma. Radiother Oncol 2012;104:312-6.

88. Zhou X, Ou X, Xu T, Wang X, Shen C, Ding J, et al. Effect of Dosimetric Factors on Occurrence and Volume of Temporal Lobe Necrosis Following Intensity Modulated Radiation Therapy for Nasopharyngeal Carcinoma: A Case-Control Study. Int J Radiat Oncol 2014;90:261-9.

89. McDonald MW, Linton OR, Calley CSJ. Dose-Volume Relationships Associated With Temporal Lobe Radiation Necrosis After Skull Base Proton Beam Therapy. Int J Radiat Oncol 2015;91:261-7.

90. Pehlivan B, Ares C, Lomax AJ, Stadelmann O, Goitein G, Timmermann B, et al. Temporal lobe toxicity analysis after proton radiation therapy for skull base tumors. Int J Radiat Oncol Biol Phys 2012;83: 1432-40.

91. Douw L, Klein M, Fagel SS, van den Heuvel J, Taphoorn MJ, Aaronson NK, et al. Cognitive and radiological effects of radiotherapy in patients with low-grade glioma: long-term follow-up. Lancet Neurol 2009;8:810-8. 
92. Guimas V, Thariat J, Graff-Cailleau P, Boisselier P, Pointreau Y, Pommier P, et al. [Intensity modulated radiotherapy for head and neck cancer, dose constraint for normal tissue: Cochlea vestibular apparatus and brainstem.. Cancer Radiother 2016;20:475-83.

93. Jian JJ-M, Cheng SH, Tsai SY-C, Yen K-CL, Chu N-M, Chan K-Y, et al. Improvement of local control of T3 and T4 nasopharyngeal carcinoma by hyperfractionated radiotherapy and concomitant chemotherapy. Int J Radiat Oncol Biol Phys 2002;53:344-52.

94. Uy NW, Woo SY, Teh BS, Mai WY, Carpenter LS, Chiu JK, et al. Intensity-modulated radiation therapy (IMRT) for meningioma. Int J Radiat Oncol Biol Phys 2002;53:1265-70.

95. Schoenfeld GO, Amdur RJ, Morris CG, Li JG, Hinerman RW, Mendenhall WM. Patterns of Failure and Toxicity after Intensity-Modulated Radiotherapy for Head and Neck Cancer. Int J Radiat Oncol 2008;71:377-85.

96. Zheng Y, Han F, Xiao W, Xiang Y, Lu L, Deng X, et al. Analysis of late toxicity in nasopharyngeal carcinoma patients treated with intensity modulated radiation therapy. Radiat Oncol 2015;10:17.

97. Weber DC, Malyapa R, Albertini F, Bolsi A, Kliebsch U, Walser M, et al. Long term outcomes of patients with skull-base low-grade chondrosarcoma and chordoma patients treated with pencil beam scanning proton therapy. Radiother Oncol 2016;120:169-74.

98. Nishimura H, Ogino T, Kawashima M, Nihei K, Arahira S, Onozawa M, et al. Proton-Beam Therapy for Olfactory Neuroblastoma. Int J Radiat Oncol 2007;68:758-62.

99. Noël G, Feuvret L, Calugaru V, Dhermain F, Mammar H, Haie-Méder C, et al. Chordomas of the base of the skull and upper cervical spine. One hundred patients irradiated by a 3D conformal technique combining photon and proton beams. Acta Oncol (Madr) 2005;44:700-8.

100. Debus J, Hug EB, Liebsch NJ, O'Farrel D, Finkelstein D, Efird J, et al. Brainstem tolerance to conformal radiotherapy of skull base tumors. Int J Radiat Oncol Biol Phys 1997;39:967-75.

101. Debus J, Hug EB, Liebsch NJ, O'Farrel D, Finkelstein D, Efird J, et al. Dose-volume tolerance of the brainstem after high-dose radiotherapy. Front Radiat Ther Oncol 1999;33:305-14.

102. Burman C, Kutcher GJ, Emami B, Goitein M. Fitting of normal tissue tolerance data to an analytic function. Int J Radiat Oncol Biol Phys 1991;21:123-35.

103. Merchant TE, Chitti RM, Li C, Xiong X, Sanford RA, Khan RB. Factors associated with neurological recovery of brainstem function following postoperative conformal radiation therapy for infratentorial ependymoma. Radiat Oncol Biol n.d.;76:496-503.

104. Greene-Schloesser D, Robbins ME, Peiffer AM, Shaw EG, Wheeler KT, Chan MD. Radiation-induced brain injury: A review. Front Oncol 2012;2:73.

105. Makale MT, McDonald CR, Hattangadi-Gluth JA, Kesari S. Mechanisms of radiotherapy-associated cognitive disability in patients with brain tumours. Nat Rev Neurol 2016;13:52-64.

106. Connor M, Karunamuni R, McDonald C, White N, Pettersson N, Moiseenko V, et al. Dose-dependent white matter damage after brain radiotherapy. Radiother Oncol 2016;121:209-16.

107. Deng W, Aimone JB, Gage FH. New neurons and new memories: how does adult hippocampal neurogenesis affect learning and memory? Nat Rev Neurosci 2010;11:339-50.

108. Gondi V, Hermann BP, Mehta MP, Tomé WA. Hippocampal Dosimetry Predicts Neurocognitive Function Impairment After Fractionated Stereotactic Radiotherapy for Benign or Low-Grade Adult Brain Tumors. Int J Radiat Oncol 2013;85:348-54.

109. Seibert TM, Karunamuni R, Bartsch H, Kaifi S, Krishnan AP, Dalia Y, et al. Radiation Dose-Dependent Hippocampal Atrophy Detected With Longitudinal Volumetric Magnetic Resonance Imaging. Int J Radiat Oncol 2017;97:263-9.

110. Eekers DBP, in 't Ven L, Deprez S, Jacobi L, Roelofs E, Hoeben A, et al. The posterior cerebellum, a new organ at risk? Clin Transl Radiat Oncol 2018;8:22-6.

111. Merchant TE, Sharma S, Xiong X, Wu S, Conklin H. Effect of cerebellum radiation dosimetry on cognitive outcomes in children with infratentorial ependymoma. Int J Radiat Oncol Biol Phys 2014;90:547-53.

112. Hamilton CS, Potten CS, Denham JW, O'Brien PC, Kron T, Ostwald P, et al. Response of human hair cortical cells to fractionated radiotherapy. Radiother Oncol 1997;43:289-92.

113. Stram DO, Mizuno S. Analysis of the DS86 atomic bomb radiation dosimetry methods using data on severe epilation. Radiat Res 1989;117:93-113. 
114. Lawenda BD, Gagne HM, Gierga DP, Niemierko A, Wong WM, Tarbell NJ, et al. Permanent alopecia after cranial irradiation: dose-response relationship. Int J Radiat Oncol Biol Phys 2004;60:879-87.

115. Langendijk JA, Lambin P, De Ruysscher D, Widder J, Bos M, Verheij M. Selection of patients for radiotherapy with protons aiming at reduction of side effects: the model-based approach. Radiother Oncol 2013;107:267-73.

116. Widder J, van der Schaaf A, Lambin P, Marijnen CAM, Pignol J-P, Rasch CR, et al. The Quest for Evidence for Proton Therapy: Model-Based Approach and Precision Medicine. Int J Radiat Oncol 2016;95:30-6.

117. Milano MT, Constine LS, Okunieff P. Normal Tissue Tolerance Dose Metrics for Radiation Therapy of Major Organs. Semin Radiat Oncol 2007;17:131-40.

118. Eekers DB, Lambrecht M, De Witt Nyström P, Swinnen A, Wesseling F, Hoffman, Aswin L, et al. EPTN consensus-based guideline for the tolerance dose per fraction of organs at risk in the brain. CancerData 2018. doi:10.17195/candat.2018.01.1.

119. Hoffmann AL, Nahum AE. Fractionation in normal tissues: the $(\alpha / \beta)$ eff concept can account for dose heterogeneity and volume effects. Phys Med Biol 2013;58:6897-914.

120. Perkó Z, Bortfeld TR, Hong TS, Wolfgang J, Unkelbach J. Derivation of mean dose tolerances for new fractionation schemes and treatment modalities. Phys Med Biol 2018;63(3):035038.

121. Report 83: Prescribing, Recording and Reporting Photon-beam Intensity-Modulated Radiation Therapy (IMRT). J ICRU 2010;10:NP.1-NP. doi:10.1093/jicru/10.1.Report83.

122. Menzel H-G. International Commission on Radiation Units and Measurements. J Int Comm Radiat Units Meas 2014;14:1-2.

123. Cox JD, Stetz J, Pajak TF. Toxicity criteria of the radiation therapy oncology group (RTOG) and the european organization for research and treatment of cancer (EORTC). Pergamon Int J Radi Ati Oncol Biol Phys 1995;3:34-1346.

124. Fromme EK, Eilers KM, Mori M, Hsieh Y-C, Beer TM. How Accurate Is Clinician Reporting of Chemotherapy Adverse Effects? A Comparison With Patient-Reported Symptoms From the Quality-ofLife Questionnaire C30. J Clin Oncol 2004;22:3485-90.

125. Di Maio M, Gallo C, Leighl NB, Piccirillo MC, Daniele G, Nuzzo F, et al. Symptomatic toxicities experienced during anticancer treatment: agreement between patient and physician reporting in three randomized trials. J Clin Oncol 2015;33:910-5.

126. Makale MT, McDonald CR, Hattangadi-Gluth JA, Kesari S. Every year, hundreds of thousands of patients worldwide undergo radiotherapy for primary brain tumours and for brain metastases originating from extracranial tumours. Nat Publ Gr 2016;13.

127. Seibert TM, Karunamuni R, Kaifi S, Burkeen J, Connor M, Krishnan AP, et al. Cerebral Cortex Regions Selectively Vulnerable to Radiation Dose-Dependent Atrophy. Int J Radiat Oncol 2017;97:910-8.

128. Fowler JF. The linear-quadratic formula and progress in fractionated radiotherapy. $\mathrm{Br} J$ Radiol 1989;62:679-94.

129. Paganetti H, Niemierko A, Ancukiewicz M, Gerweck LE, Goitein M, Loeffler JS, et al. Relative biological effectiveness (RBE) values for proton beam therapy. Int J Radiat Oncol Biol Phys 2002;53:407-21.

130. Paganetti $\mathrm{H}$, van Luijk P. Biological Considerations When Comparing Proton Therapy With Photon Therapy. Semin Radiat Oncol 2013;23:77-87.

131. Indelicato DJ, Flampouri S, Rotondo RL, Bradley JA, Morris CG, Aldana PR, et al. Incidence and dosimetric parameters of pediatric brainstem toxicity following proton therapy. Acta Oncol (Madr) 2014;53: 1298-304.

132. Gunther JR, Sato M, Chintagumpala M, Ketonen L, Jones JY, Allen PK, et al. Imaging Changes in Pediatric Intracranial Ependymoma Patients Treated With Proton Beam Radiation Therapy Compared to Intensity Modulated Radiation Therapy. Int J Radiat Oncol 2015;93:54-63.

133. Peeler CR, Mirkovic D, Titt U, Blanchard P, Gunther JR, Mahajan A, et al. Clinical evidence of variable proton biological effectiveness in pediatric patients treated for ependymoma. Radiother Oncol 2016;121:395-401. 


\section{Supplemental material}

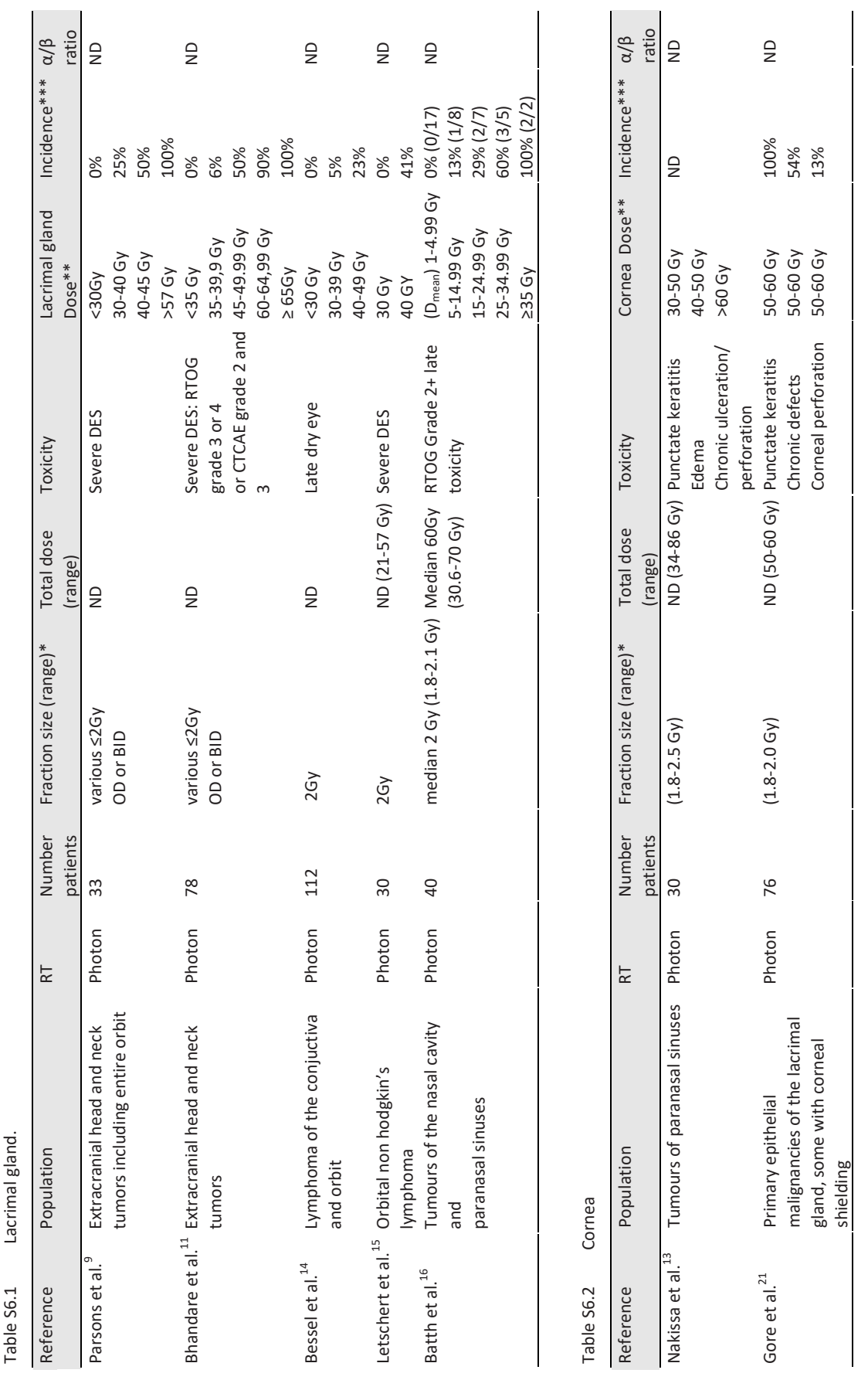


Neuro-Oncology organs at risk dose constraints

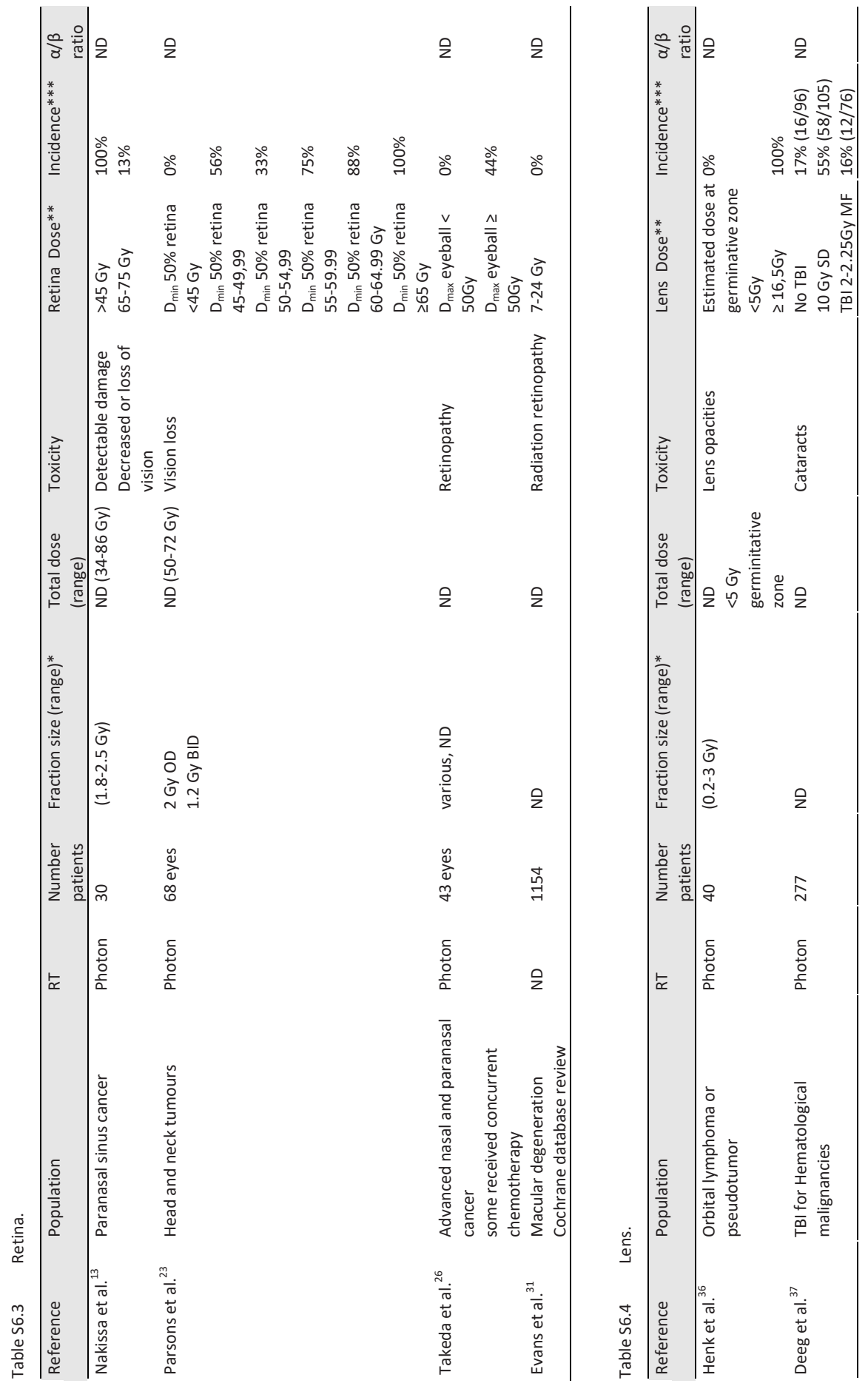




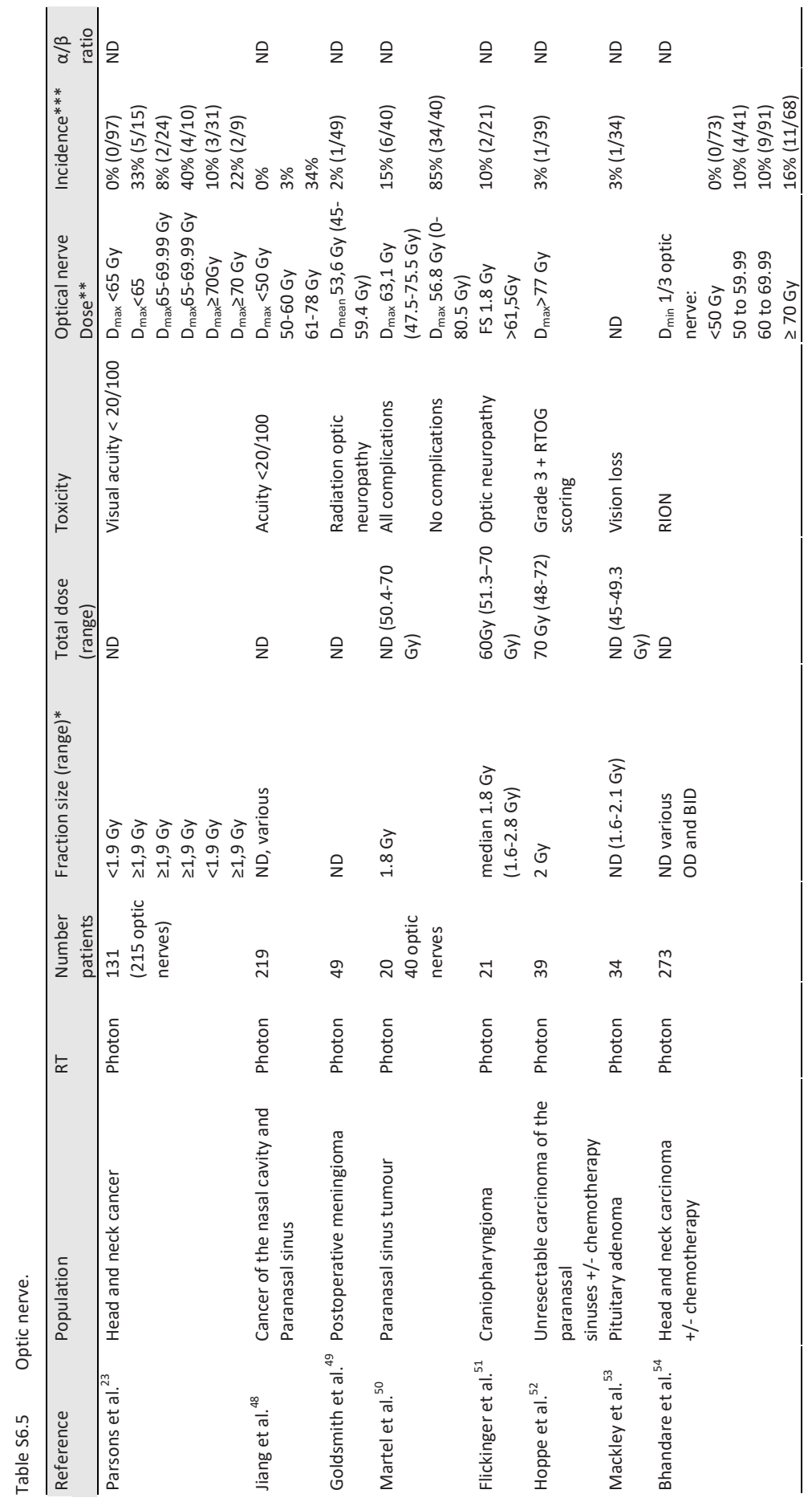


Neuro-Oncology organs at risk dose constraints

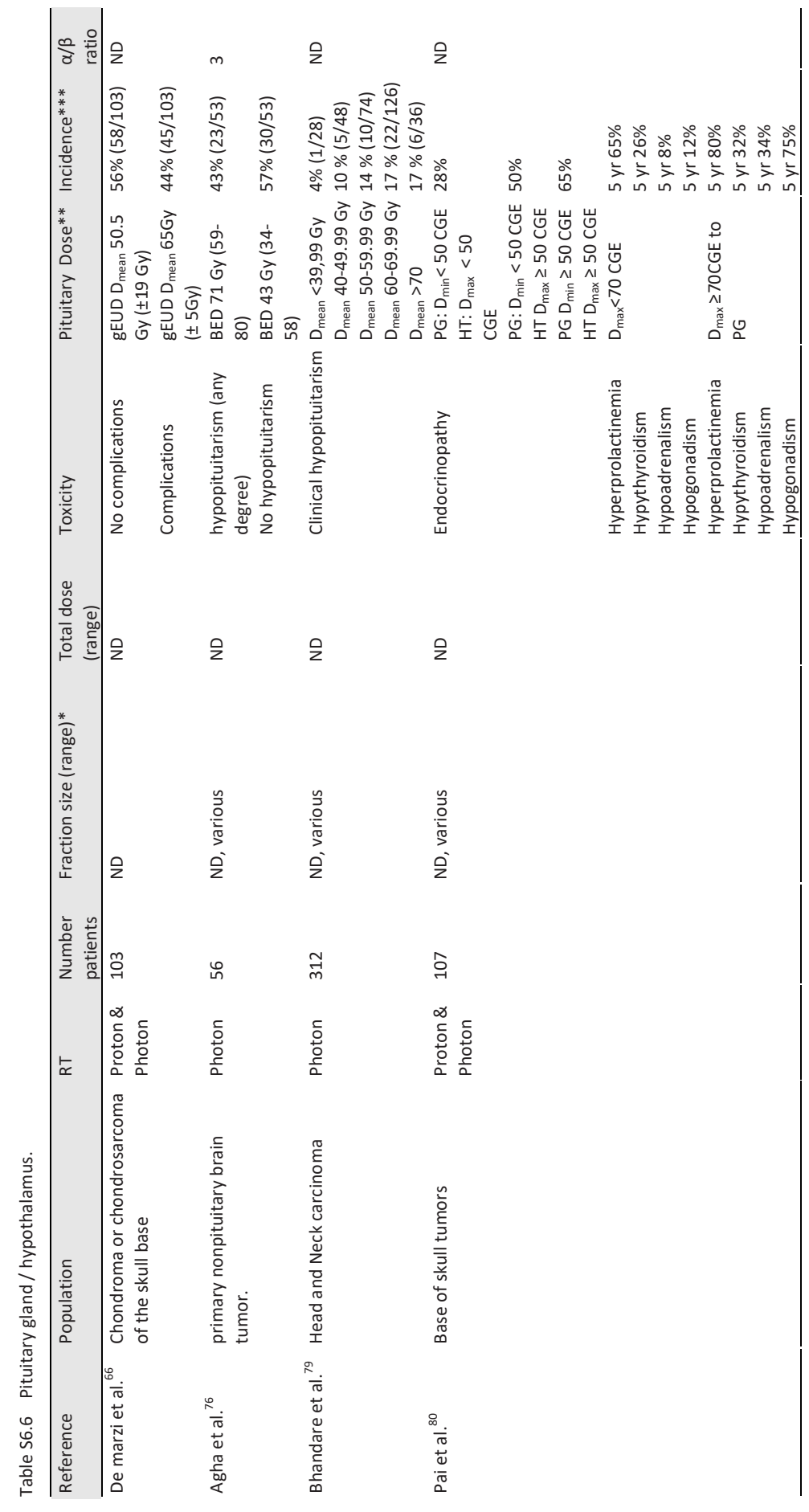


Chapter 6

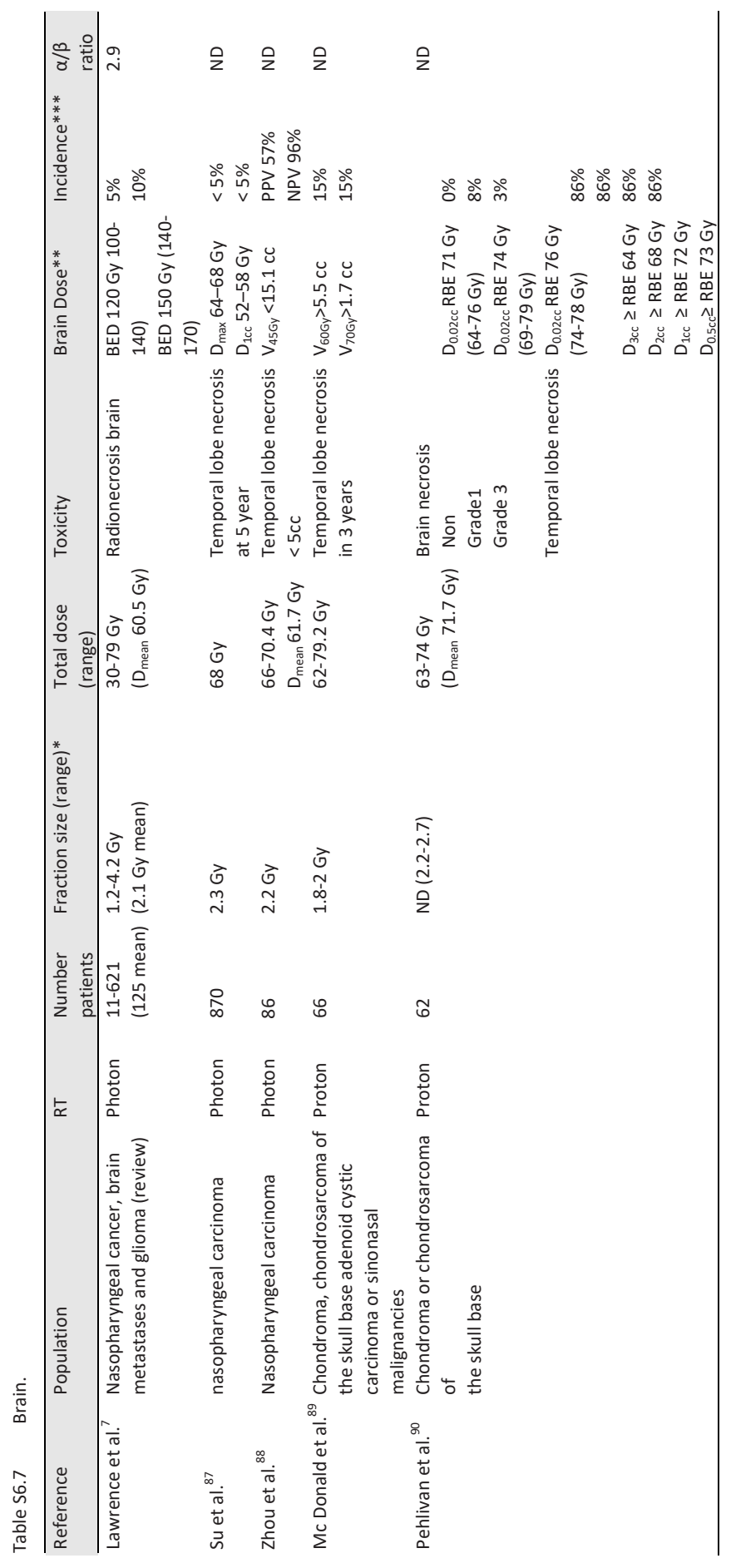


Neuro-Oncology organs at risk dose constraints

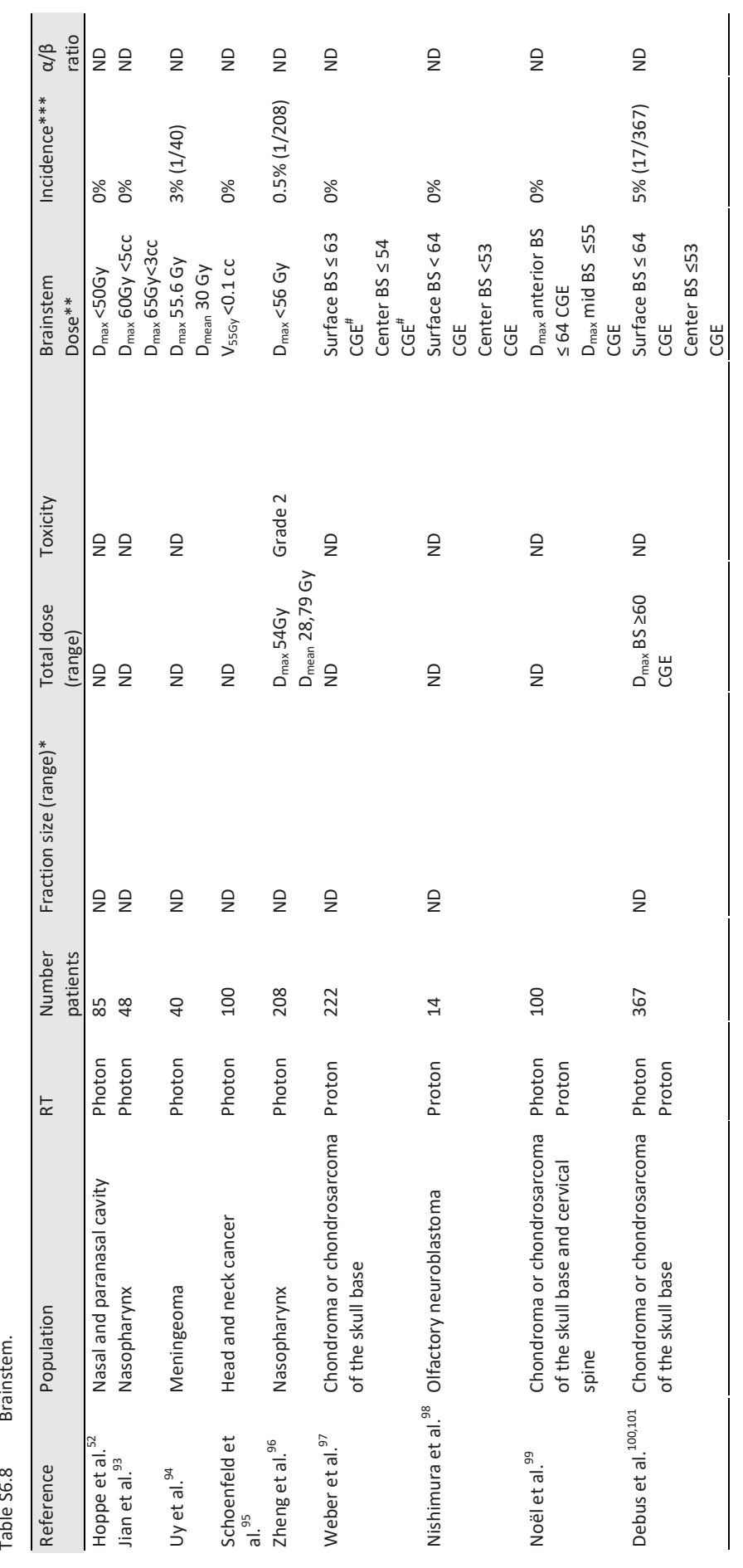


Chapter 6

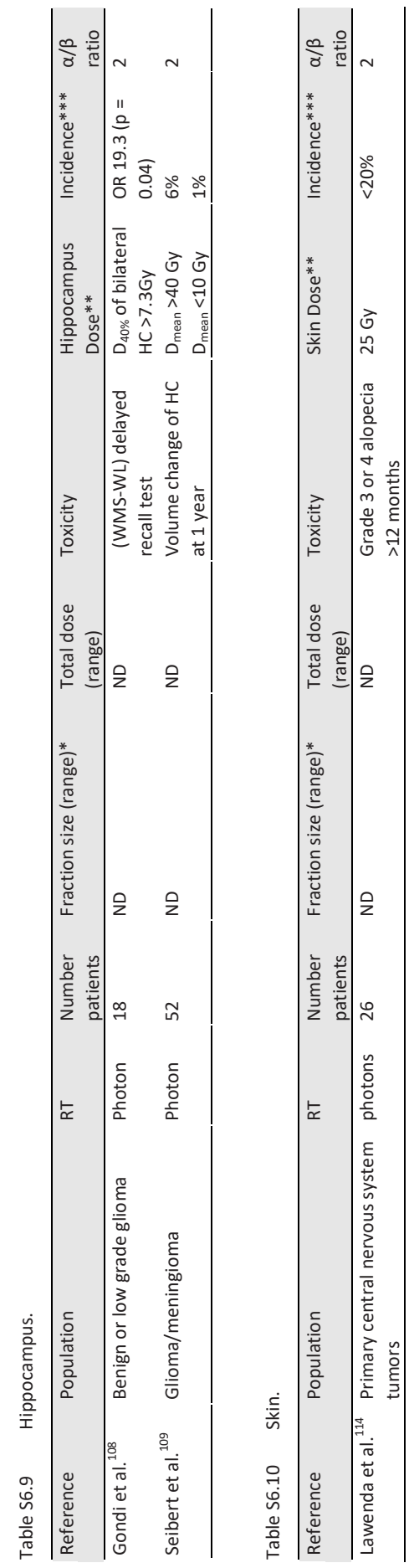




\section{Chapter 7}

Evidence on the efficacy of primary radiosurgery or stereotactic radiotherapy for resistant nonneoplastic focal epilepsy in adults: a systematic review

Daniëlle B.P. Eekers, Esther N. Pijnappel, Olaf E.M.G. Schijns, Albert Colon, Ann Hoeben, Jaap D. Zindler, Alida A. Postma, Aswin L. Hoffmann, Philippe Lambin, Esther G.C.Troost 


\section{Abstract}

\section{Purpose}

Although the majority of adult epilepsy patients respond well to the current antiepileptic drug treatment, $20-40 \%$ of them are drug-resistant. In these patients, resective epilepsy surgery is a curative treatment option, for which, however, only a limited number of patients is eligible. The purpose is to summarize the outcome of radiotherapy for drug-resistant non-neoplastic focal epilepsy and to elucidate its efficacy for seizure outcome and long-term toxicity in adults.

\section{Method}

A systematic literature search was performed in Pubmed, Ovid Medline, Cochrane library, Embase and Web of Science. The methodological quality was evaluated using an adapted QUADAS checklist.

\section{Results}

Sixteen out of 170 initially identified studies were included in this systematic literature study ( $n=170$ patients). Twelve of the 16 studies described a positive effect of radiotherapy on seizure frequency reduction, with 98 of the patients (on average $58 \%$, range $25 \%-95 \%$ ) reporting no or rare seizures (defined as radiotherapy-adapted Engel class [RAEC] I and II. In total, 20\% (34 patients) of the patients needed subsequent surgery due to radionecrosis, cysts formation, edema, and intracranial hypertension or remaining seizures. A dose-effect model was fitted to the available response data in an attempt to derive a relationship between prescribed dose and RAEC frequency.

\section{Conclusions}

Radiotherapy is a possible non-invasive treatment option for patients with drugresistant focal non-neoplastic epilepsy. This systematic review showed that there is only level 4 evidence of primary radiotherapy reducing seizure frequency in adult patients. Prospective randomized trials are needed to determine its exact value compared to other treatment approaches. 


\section{Introduction}

Epilepsy is one of the most common severe neurological disorders. The World Health Organization has estimated that more than 50 million patients suffer from epilepsy worldwide ${ }^{1-3}$.

According to the International League Against Epilepsy several types of epilepsy exist ${ }^{4,5}$. In this review we focus on non-neoplastic focal epileptic lesions (NNFE), associated with focal seizures, which have a localized, well-circumscribed network of discharges ${ }^{6}$.

Non-neoplastic lesions, that have been described by the European Epilepsy Brain Bank consortium as the pathological substrate of focal seizures include, in descending order of frequency, hippocampal sclerosis (36.4\%), long-term epilepsy-associated tumors (23.6\%), malformations of cortical development (19.8\%), vascular malformations (6.1\%) and glial scars $(4.8 \%)$ as well as no lesion $(7.7 \%)^{7}$. The most frequent non-neoplastic lesions of drug-resistant focal epilepsies, constituting about $80 \%$ of all resective epilepsy surgery cases, are hippocampal sclerosis and malformations of cortical development besides long-term epilepsy-associated tumors ${ }^{7}$. In the group of low-grade epilepsy associated tumors, some of these tumors can, although very rare, dedifferentiate into high-grade neoplastic subforms $\mathrm{s}^{8-10}$. This is one reason why we focus on hippocampal sclerosis and malformations of cortical development in this review. A second reason is that almost all well-documented clinical series of focal epilepsy and radiosurgery include patients with tumors. Since the beginning of the 21st century, studies are emerging describing non-space-occupying lesions, mainly hippocampal sclerosis, with long-term follow-up ${ }^{11-15}$. Only very recently, studies on the radiosurgical treatment of periventricular heterotopias and focal cortical dysplasias have been published, although with a short follow-up period of the treated patients ${ }^{16,17}$.

The type and origin of epilepsy determines the prognosis and the efficacy of the treatment. Currently, the two most frequently used therapeutic options include antiepileptic drug (AED) therapy and resective epilepsy surgery. Still resective epilepsy surgery is underused globally and patients tend to be referred with a long delay ${ }^{18,19}$. Noteworthy, postoperative seizure free outcome varies between $60-90 \%$ depending, among others, on the pathological substrate ${ }^{20}$.

The third, less frequently used therapeutic option is radiation therapy (RT). This noninvasive approach may be superior to surgery when the epileptogenic region is located near the eloquent cortex or deeply sited brain areas. In theory, RT may achieve a better neurotransmitter equilibrium than resective epilepsy surgery, and thus result in better neuropsychological outcome despite the late response effects ${ }^{20,21}$. Stereotactic RT (SRT) is a high-precision three-dimensional external beam radiation therapy technique directing beams to a well-defined target, relying on detailed imaging and precise 
treatment set-up to deliver the radiation dose while sparing the surrounding normal tissue. By using multiple fractions (so-called fractionated) SRT, the dose is delivered in multiple sessions over a longer period of time, instead of a single-session large dose, also referred to as stereotactic radiosurgery (SRS). Fractionated SRT has been proven to be superior to SRS when considering tolerance dose of normal brain tissue and cranial nerves and thus higher (radio-)biologically equivalent doses can safely be delivered using the former 22 .

Since long-term side effects of RT may comprise the induction of secondary tumors and growth delay in children, we focused on adult patients in this review. So far, several publications have underlined the potential value of RT in patients with drug-resistant epilepsy ${ }^{18-20,23}$. To date, however, no systematic review has been published on the efficacy of RT in adults patients with drug-resistant FNNE, excluding bias effects of previous resection. Therefore, this systematic review summarizes the available evidence for efficacy (no or rare seizures in Engel class I\&II patients ${ }^{24}$ ) and treatmentrelated side effects in patients with focal drug-resistant epilepsy undergoing RT.

\section{Materials and methods}

\section{Research protocol}

To develop the research protocol and identify the scope of the review, we followed a structured approach to identify the patient population, interventions, comparators, outcomes, and study design (PICOS criteria). ${ }^{25}$ As ILAE-terminology on epilepsy recently changed (Fisher 2017), search terms for epilepsy were "focal" and "localization related". The research question was defined as: what is the level of evidence on the efficacy of primary RT for drug-resistant FNNE in adults? For this, therapeutic benefit was defined as seizure frequency reduction using the modestly adapted Engel classification. We adapted only the Engel class III, which was defined as an improvement of epilepsy frequency with more than 75\% (Figure 7.1). Therapy-induced complications were defined as late radiation damage. The research protocol contained a comprehensive search strategy and screening criteria for abstracts, titles and full text articles.

\section{Eligibility criteria, search strategy and study selection}

A comprehensive search was performed to identify the current papers in PubMed (National Center for Biotechnology Information, NCBI), Ovid Medline, Cochrane library, Embase and Web of Science. PubMed was used as the primary data source; Ovid 
Medline, Cochrane library, Embase and Web of Science were used to extract additionally available articles (Supplementary Table S7.1 and S7.2). These search strategies were frequently performed with the last search in May, 2017. To finalize the systematic literature search all included articles' reference lists were crossed-checked (citation tracking) on potential relevant studies that met the inclusion criteria.

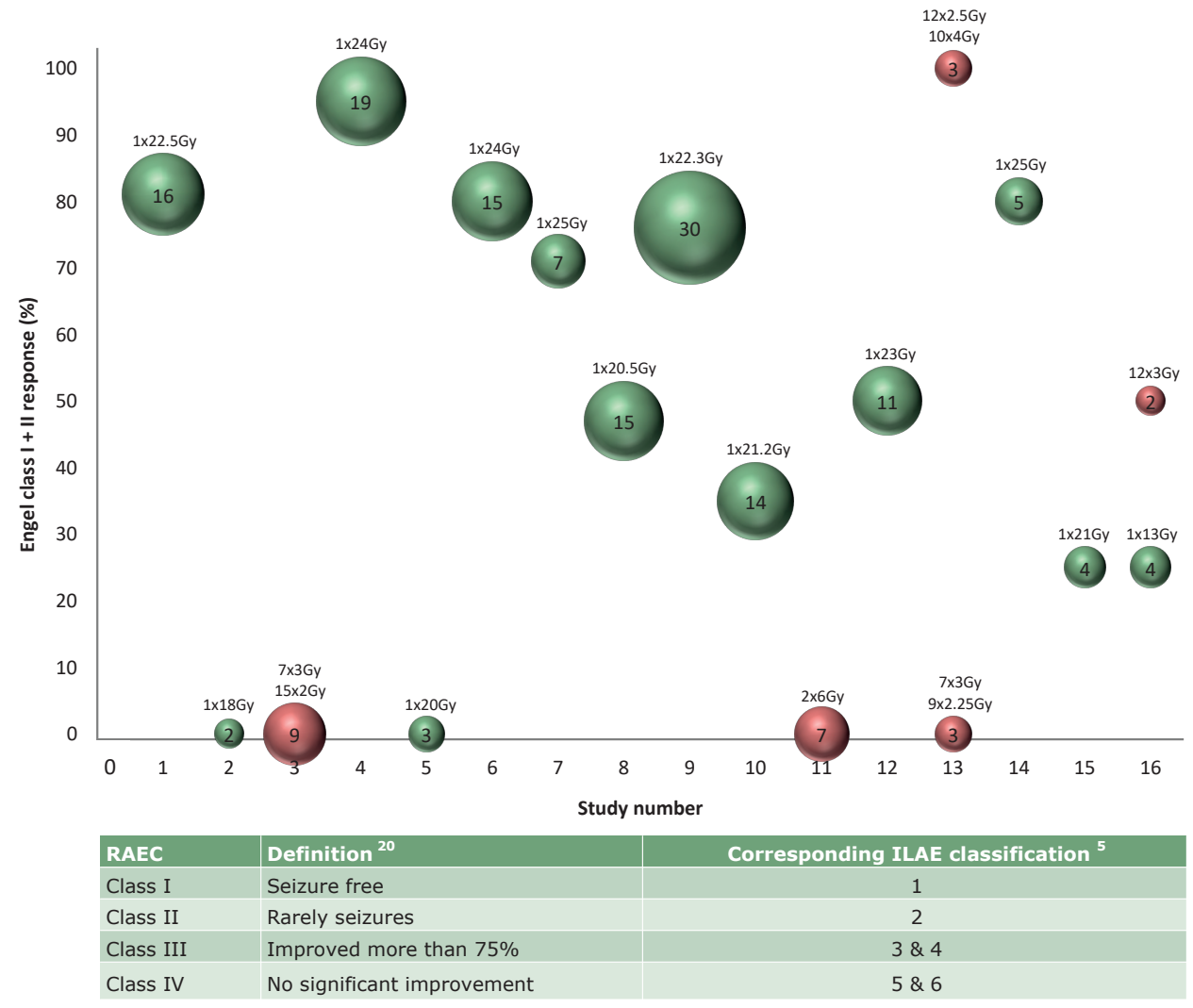

Figure 7.1 Percentage patients with Engel class I+II. Top: The percentage of patients with Radiotherapy adapted Engel classification (RAEC) I+II for the 16 studies. On the horizontal axis, the study numbers is given (corresponding to Table 7.1), while on the vertical axis the percentage of patients is plotted. The numbers given in, and the size of the respective bubble indicate the number of patients included in each study. The green colour indicates a single fraction, while red highlights multiple fractions. The prescribed dose (number of fractions times the mean fraction dose) is given above each bubble. Bottom: The Radiotherapy adapted Engel classification (RTAEC) used to define response to radiation treatment translated to International League Against Epilepsy (ILAE) classification.

After literature collection, completed abstracts and titles were first assessed on identical records and duplicate findings were removed from the search. In addition, 
identical patient cohorts were only entered once, comprising the study with most information on patient characteristics, seizure outcome and the longest follow-up period. Abstracts and full text articles were assessed using inclusion criteria and exclusion criteria (Supplementary Table S7.3). Two investigators (EP and DE) established the research protocol and independently performed the literature searches. A third independent reviewer (ET) was consulted if no agreement was reached amongst the two investigators.

\section{Data collection}

Data collection was performed with the use of a well-defined questionnaire in Microsoft Excel (Supplementary Table S7.4). Unfortunately, we were unable to perform a meta-analysis for controls since studies were not designed with control groups.

\section{Data quality assessment and risk of bias within studies}

To assess the methodological quality of the included studies the QUADAS-2 checklist was intended to be used. ${ }^{24}$ However, most domains (patient selection, index test, reference standard and flow and timing) of the QUADAS checklist were not applicable to our research. ${ }^{24}$ Mainly the index test, which in our study would have been a control group, was not described in the studies included in this review. Therefore, it was decided to use a modified version of the QUADAS checklist to assess the risk of bias and applicability to the research question of all included articles. In this way the individual quality of each included study was evaluated (Supplementary Table S7.5). For all studies information on in- and exclusion criteria, loss to follow-up, follow-up time, definition of outcome measure and exposure to radiotherapy was scored.

\section{Dose-response model}

Dose response was tested with Kendall's tau correlation ${ }^{17}$. The biological effective dose (BED) is commonly used for isoeffective dose calculations when comparing differing fractionation schedules, i.e. fraction dose and number of fractions. After converting the physical dose to BED using an $\alpha / \beta$ of $10 \mathrm{~Gy}\left(\mathrm{BED}_{10}\right)$, a logistic probability density function (logit model) was fitted by the least-square method:

$$
P(D)=\frac{1}{1+\left(\frac{D_{50}}{D}\right)^{4 V_{50}}}
$$

where $D=B E D_{10}, D_{50}$ is the $\mathrm{BED}_{10}$ at $50 \%$ response, and $\gamma_{50}$ is the normalized slope at $D_{50}$. The $95 \%$ confidence intervals $(95 \% \mathrm{Cl})$ were determined by bootstrapping. 


\section{Results}

\section{Data extraction and bias assessment}

\section{Study selection}

The study selection process complying with the PRISMA statement ${ }^{25}$ is illustrated in Figure 7.2. Sixteen out of 170 initially identified studies were included in this systematic literature study ( $n=170$ patients).
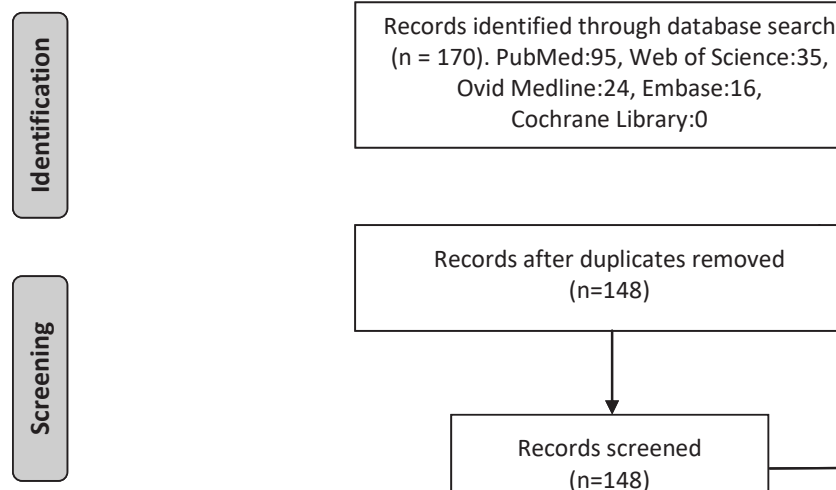

Records after duplicates removed $(n=148)$
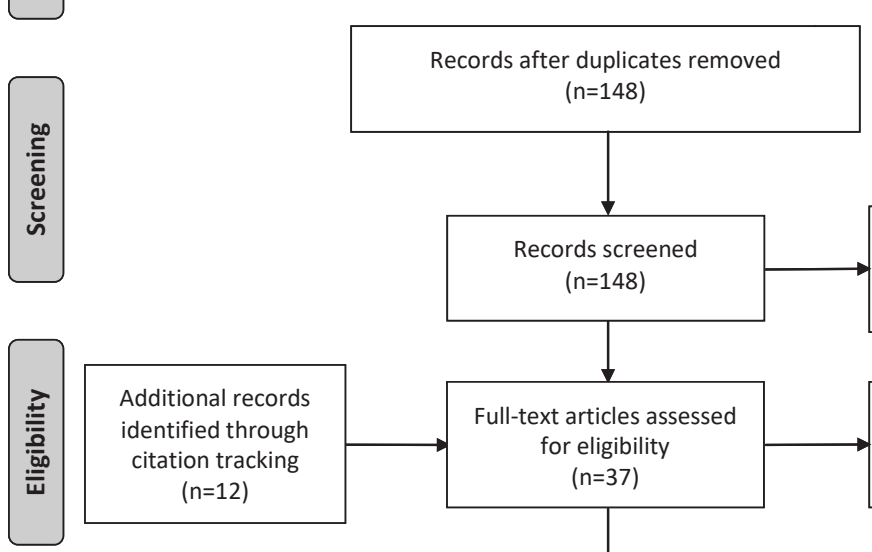

Additional records identified through citation tracking $(n=12)$

Records excluded based on title and abstract $(n=122)$

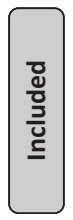

Studies included in qualitative synthesis $(n=16)$

Figure 7.2 PRISMA flow diagram ${ }^{11}$.

\section{Risk of bias within studies}

A summary of the parameters that we used to identify the risk of bias is found in Supplementary Table S7.4. Nine out of 16 studies explicitly described inclusion criteria that were carefully selected and enabled comparisons between study populations. 
However, exclusion criteria were not well defined in most publications and by comparing the seizure frequency before and after therapy, the patient was used as his/her own control. None of the studies used control groups. Therefore, selection bias could not be identified. In addition, information on the cohorts from which the study populations were drawn was not given, which hampered comparisons. A final risk of bias was the fact that the definitions of the post-treatment seizure outcome measures were not in accordance with the Engel classification in seven of the included studies, but sufficient information was provided to enable the transformation to the radiotherapy-adapted Engel class (RAEC). RAEC I was defined as seizure free, II as rarely seizures, III as an improvement of more than $75 \%$ and class IV as no significant improvement (see Figure 7.1).

\section{Patient selection in individual studies}

The most important information from each included study is presented as a framework for conclusions. In the study by Régis et al. ${ }^{11}$, patient number 11 and 18 were identical to the patients in the study by Rheims et $a .^{26}$. For reason of longer follow-up, these patients were analyzed in the latter study only. In addition, two patients discussed in the study by Usami et al. ${ }^{27}$ were identical to patients in the article of Kawai et al. ${ }^{28}$. Since more details about the patient characteristics were available in the article of Kawai et al. ${ }^{28}$ these two patients were analyzed there. In the studies by Kawamura et al. ${ }^{29}$, Usami et al. ${ }^{27}$, Barbaro et al. ${ }^{12}$, Regis et $a l .{ }^{11}$, Srikijvilaikul et $a l .{ }^{30}$, and Rheims et al. ${ }^{26}$ some patients underwent surgery or died before the end of the follow-up time. Since only Srikijavilaikul et al. ${ }^{30}$ documented in detail which specific patients underwent surgery or died, these patients could not be excluded from the systematic review in the other studies. Therefore, the effect of RT on the outcome of seizures could not be undoubtedly shown in these three included studies, posing a possible bias in treatment outcome.

\section{Data analysis}

\section{Study characteristics}

Table 7.1 shows the RT parameters of the included studies. Detailed information on the study characteristics is shown in Table 7.2. Long-term toxicity is summarized in Table 7.3. Noteworthy, the number of patients questioned regarding their subjective radiation-induced side effects is unknown for they were objectively visualized by magnetic resonance imaging. 
Focal epilepsy treated with stereotactic radiotherapy

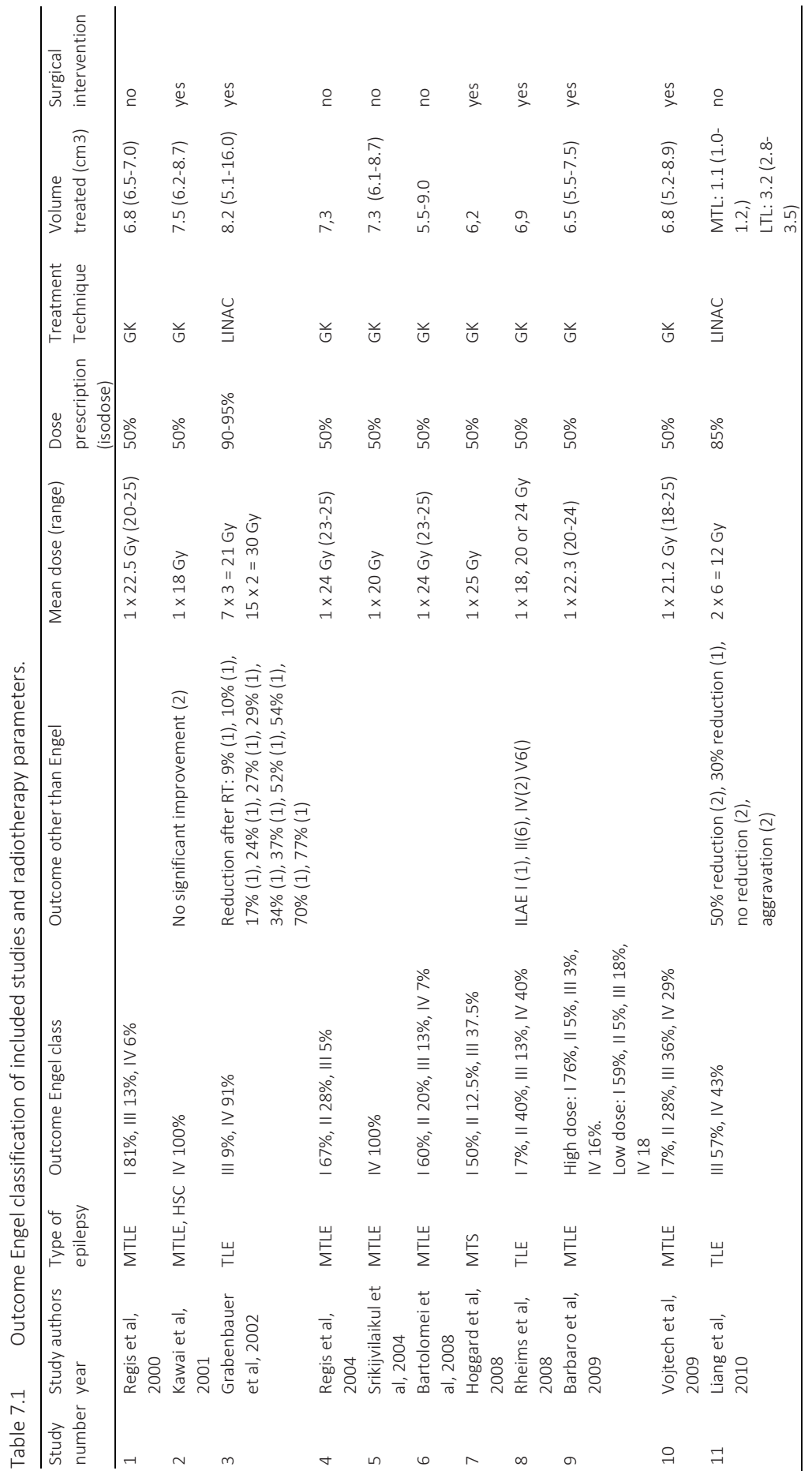


Chapter 7

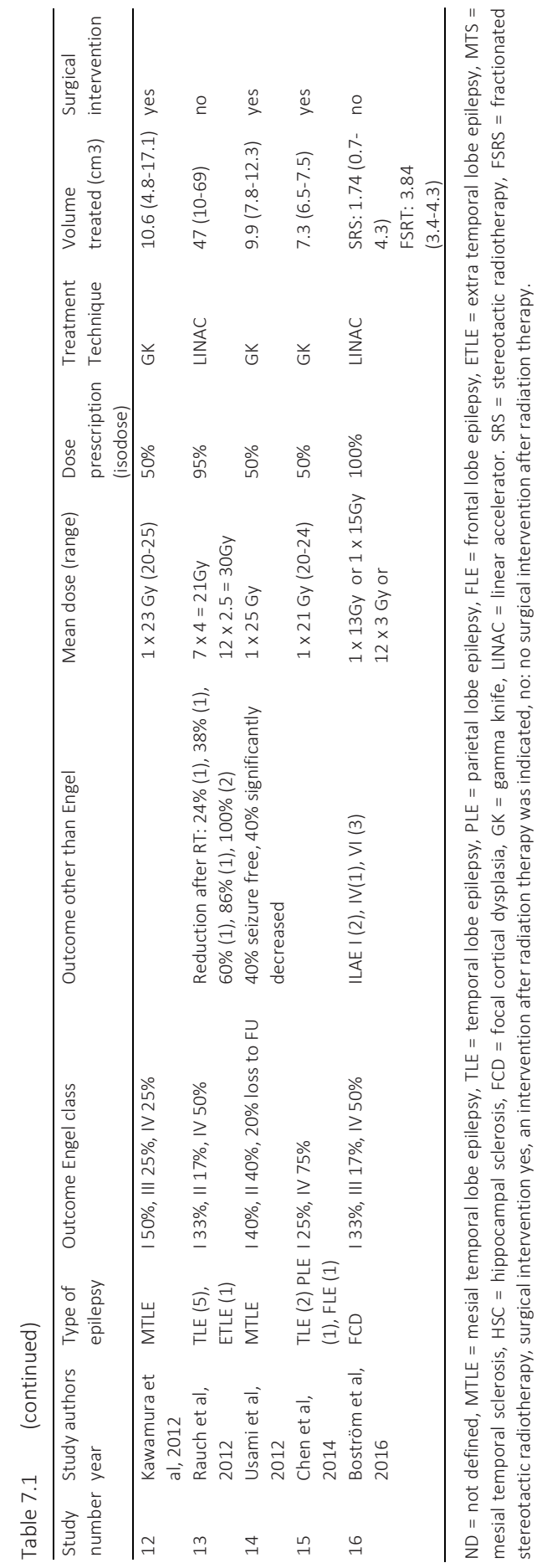




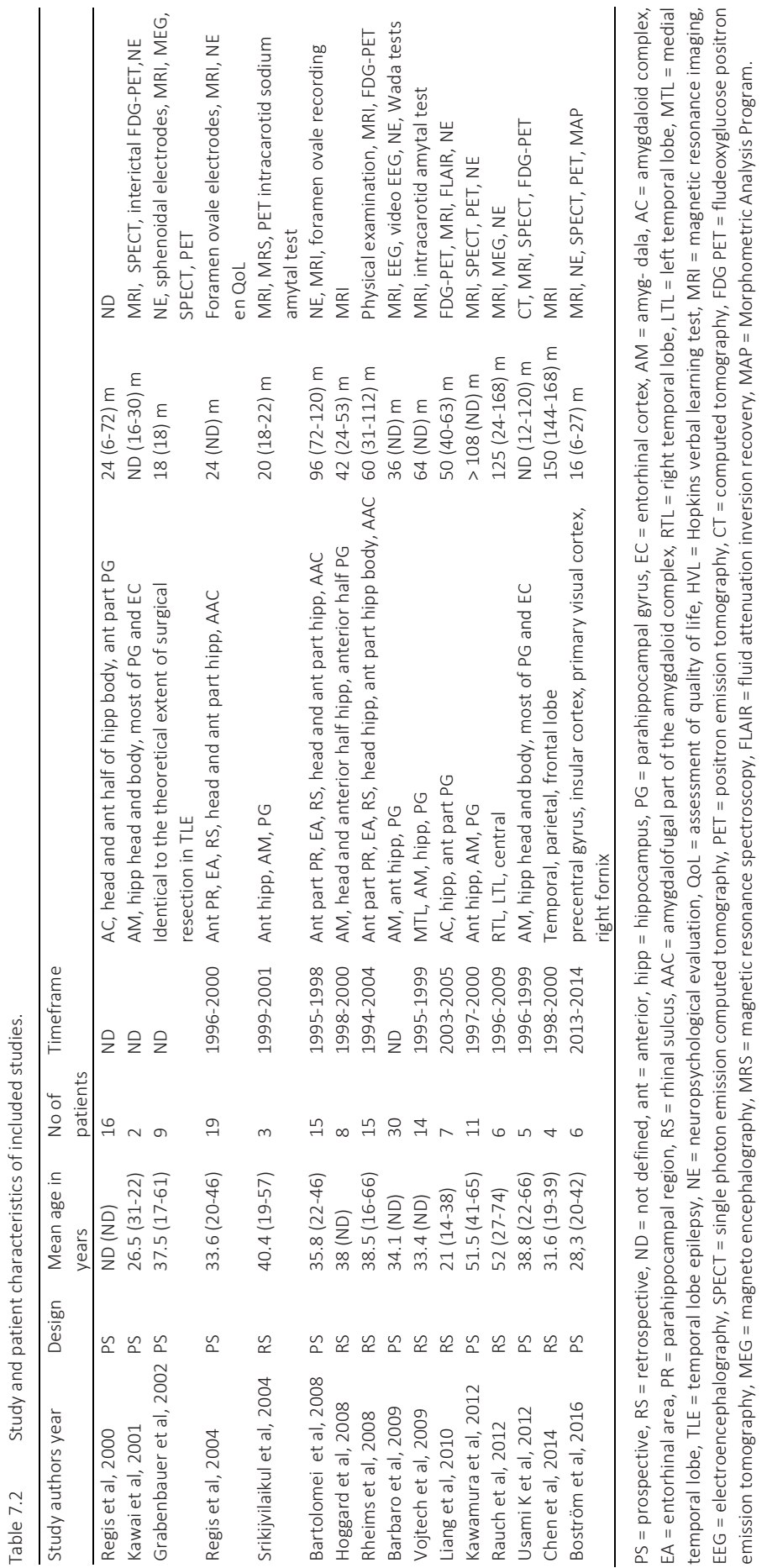




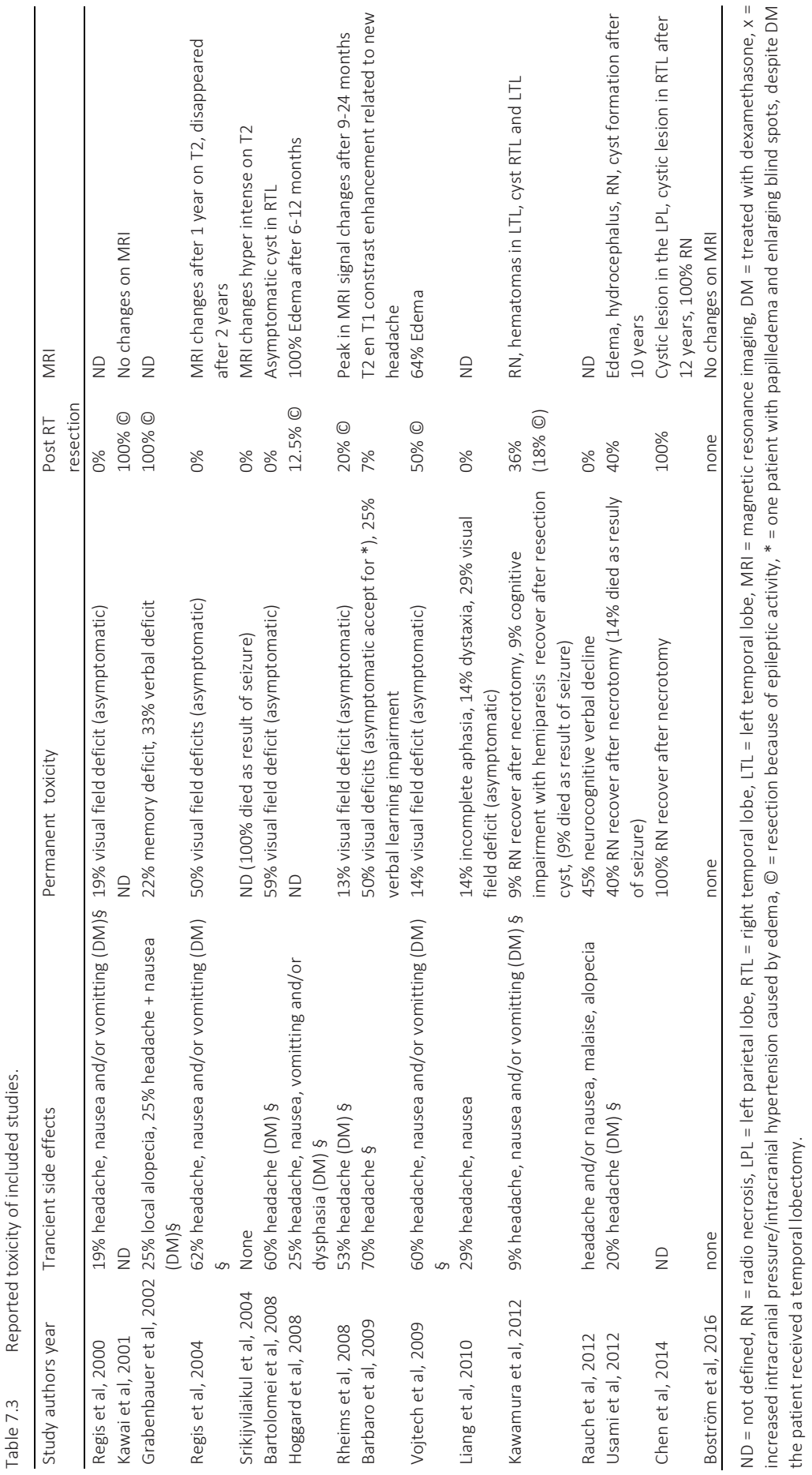




\section{Radiation therapy dose schedules and prescription}

The included studies used a wide range of dose schedules, from single fractions prescribing 13-25 Gy as marginal dose to 2, 7, 12 and 15 fractions of 6, 3, 4, 2.5, 3 and 2 Gy, respectively, prescribed to the $95 \%$ isodose (Table 7.1 ). The maximum dose in the target volume was only reported in the study of Rauch et al. ${ }^{31}$

\section{Treatment effect on seizure outcome}

As measure of efficacy of RT, the RAEC system was used in this systematic review. Most studies ( $n=9$ ) used the Engel classification system, five studies did not use any of the classification systems but provided sufficient information on seizure outcome, and two studies indicated the seizure outcome with ILAE classification. To be able to compare study outcome, the investigators independently assessed these seven studies and transformed the seizure outcome to RAEC (Figure 7.1). ${ }^{16,26-28,30-33}$ RAEC I and II were subsequently combined since these classes are both assumed to give a beneficial effect after treatment (class I no seizures; class II rare seizures; Figure 7.1) ${ }^{24}$. Twelve of the 16 studies reported a positive effect of RT on the seizure outcome defined by the total percentage of RAEC I and II patients, ranging from 25\%-95\% per study, with an overall average of $57 \%$ (98 patients). In the two smallest studies (two and three patients, respectively), RT had no favorable effect on the seizure outcome (class IV), although treatment was delivered with similar techniques, dose per fraction and target volumes compared to the other studies. ${ }^{28,30,31}$

\section{Dose-response relationship in FNNE}

RT was found to exhibit a dose effect in the RAEC $1+I$ cohorts $(r=0.69, p \leq 0.001)$. The model parameter values obtained were: $D_{50}=69.03$ [95\% Cl: 64.4-74.1] Gy and $\nu_{50}=2.16$ [95\% Cl: 1.21-6.6]. The fitted dose-response graph is illustrated in Figure 7.3. As can be gathered from the graph, a BED 10 of $69-80$ Gy is required for an RAEC I+II response in $50-75 \%$ of the patients. Furthermore, this figure suggests that higher response rates can be obtained at a lower $B E D_{10}$ for multi-fraction schemes than for single-fraction schemes. 


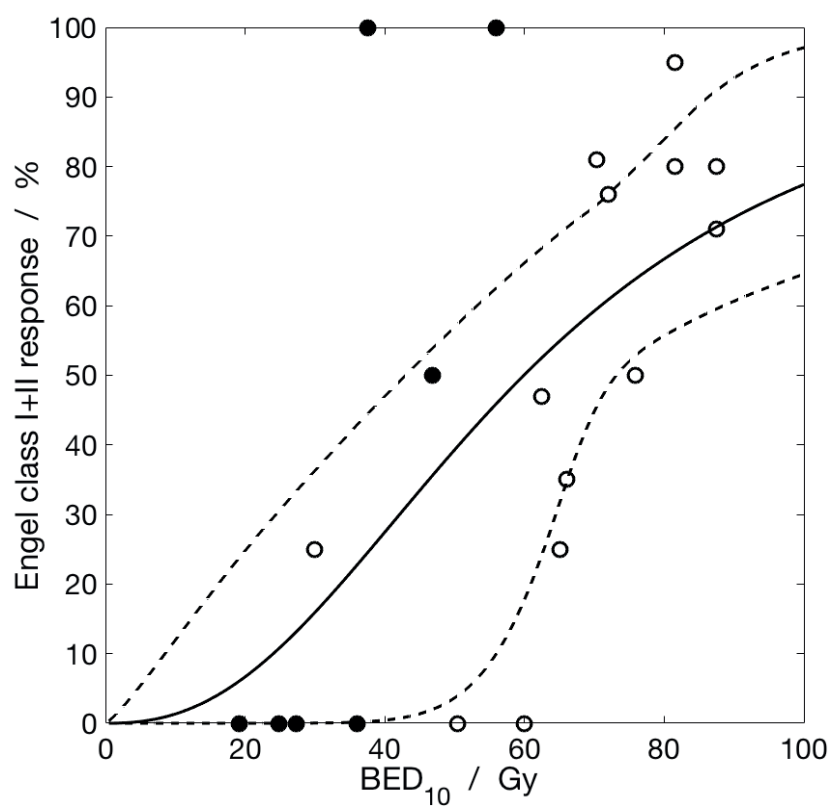

Figure 7.3 Dose response analyses. Engel class I + II rate as function of the biological effective dose using an $\alpha / B$ of $10 \mathrm{~Gy}\left(B E D_{10}\right)$, with fitted logistic probability density function (solid lines) superimposed over data points from single-fraction (open circles) and multi-fraction (closed circles) treatment schemes depicted in Figure 1. The 95\% confidence intervals (dashed line) were determined by bootstrapping.

\section{Long-term toxicity}

\section{MRI changes}

All studies reported on long-term toxicity after treatment with RT, of which 10 studies included imaging information on structural/anatomical MRI (Table 7.3). The first changes on MRI scans were observed after 6 months whereas they also occurred 10 years following RT. ${ }^{28}$ Hyperintense regions on the T2-weighted MRI scans were first detected after 6 months (peak 9-24 months) and tended to disappear after a few years, whereas four studies described cystic lesions several years after treatment which needed resection. ${ }^{11-14,27,29,30,32,34,35}$

\section{Surgical intervention}

Overall, 20\% (34 patients; range 7-100\%) of the patients in nine of the 16 studies underwent subsequent surgery. ${ }^{12-14,26-29,32,34,35}$ In six of these studies resection was performed because of persisting seizures, mostly within a 2 years follow up period, in 
those patients in whom AEDs could not control the epilepsy. ${ }^{26,28,29,32,34,36}$ In all studies, in which MRI was performed, the symptoms that were described by the patient were corresponding to an alteration visible on MRI scans. In three studies treating with SRS (21, 23 and $25 \mathrm{~Gy})$, radionecrosis was reported to have occurred requiring subsequent surgery 7-12 years following treatment. ${ }^{13,27,29}$ In one study, one patient underwent surgery without any abnormalities seen on MRI. ${ }^{12}$ Noteworthy, following fractionated treatment schedules, no patient required surgical resection or developed radionecrosis.

\section{Neurocognitive functioning}

Some of the studies reported on cognitive functions. Grabenbauer et al. ${ }^{32}$ treated nine patients with temporal lobe epilepsy and found post-treatment memory deficit in $2 / 9$ (22\%) and verbal deficit in 3/9 (33\%) patients. In the second study from Rauch et al. ${ }^{31}$, verbal decline was found in $45 \%$ of the treated patients for temporal lobe epilepsy and extratemporal lobe epilepsy. Quigg et $a .^{37}$ published the neuropsychological outcome of the article by Barbaro et $a .^{12}$, in which they concluded that there was no difference from baseline regarding language, verbal memory, cognitive efficiency and mental flexibility, nor mood. Conversely, QOL scores improved at 24 and 36 months, with those patients attaining seizure remission by month 24 accounting for the majority of the improvement. Regis et al. ${ }^{11}$ observed no neuropsychological deterioration 2 years after treatment, reporting the quality of life being significantly better than that before surgery. Vojtěch et $a{ }^{36}{ }^{36}$ published their neuropsychological results in a separate article in 2015 , in which they reported no significant changes in memory or intelligence at two years after radiosurgery ${ }^{38}$. The effect on daily functioning has not been stated. As Taphoorn et al. ${ }^{39}$ described, AED itself are more strongly associated with cognitive deficits than RT.

\section{Miscellaneous side-effects}

Headache, nausea and/or vomiting related to increased intracranial pressure and edema on MRI, were reported in 12/16 studies and easily treated with corticosteroids. The moment of onset and duration of these complaints was not described in detail. ${ }^{11-}$ 13,26,27,29,31,32-36 Visual field deficits, i.e., quadrantanopia and hemianopia, were reported in 6/16 studies with an incidence of $14-59 \% .{ }^{11-14,33,36}$ Strikingly, quadrantanopia was also found in the study of Liang et al. ${ }^{33}$ after administration of 2 fractions of $6 \mathrm{~Gy}$, a total dose not generally considered to cause visual deficits. 


\section{Discussion}

The optimum treatment for mesial temporal lobe epilepsy would be eliminating seizures with low risk, yet sparing the hippocampal function and surrounding brain. When no neoplastic lesion is present it was hypothesized by Régis et al. ${ }^{23}$ that single fraction stereotactic radiation therapy is inducing changes in the functioning of the neural tissue, by inducing remodeling of the glial environment, and is leading to the modulation of function while preserving basic processing. Chang et al. ${ }^{15}$ proposed progression of vascular injury and tissue necrosis following radiotherapy to be associated with a remission of seizures. Barbaro et al. ${ }^{12}$ randomized patients by dose; they demonstrated that the higher dose arm had better outcomes (within statistical power limits). This same effect was found in other small series underlining the tendency of higher doses to have a better antiepileptic effect than lower doses ${ }^{40-43}$. Maesawa et al. ${ }^{44}$ showed in a rat model, that SRS controlled epilepsy accompanied by stabilization of memory compared with untreated epileptic rats without causing subsequent behavioral impairment, for high (60 Gy) as well as low dose (30 Gy), supporting the hypothesis that even this relatively lower dose RT could be effective. The lowest listed radiosurgical single dose is $13 \mathrm{~Gy}$ as a marginal dose (see Figure 7.1).

Also outside of the mesial temporal structures, crucial for a successful seizure outcome after resective epilepsy surgery or SRS is to define and precisely delineate the seizure onset zone (SOZ) or the so-called focal epileptic generator. There is a worldwide growing tendency to consider the potential epileptogenic network as important as the focal lesion on MRI. As clearly illustrated by Ladino et al. ${ }^{45}$ and Stefan et al. ${ }^{46}$, heterotopia associated seizures can arise from the heterotopia alone (= focus) but can also be generated from overlying and surrounding cortex, another distant heterotopic nodule or even a dual pathology lesion, like hippocampal sclerosis (= network theory). Before definitive treatment, open resection or SRS, is advised to the patient strong evidence must be collected whether the MRI focus or the network generates the seizures in this particular patient, e.g. by invasive electrode implantation.

\section{Summary of evidence}

This systematic review gives an overview of the available literature on the efficacy of primary RT for FNNE in adults. Due to the lack of case-control studies, the results of this study only provide level 4 evidence indicating that RT may be a therapeutic option to reduce the seizure frequency for NNFE in adults. ${ }^{47}$ When considering the low level of evidence there is an inevitable ceiling effect because the absence of "control" groups is nearly inherent in surgical studies since the demands of equipoise would not allow 
design of studies with a non-treatment arm in most US and European studies. For example the study of Wiebe et al. ${ }^{48}$ randomizing open surgery to a wait-list, could not be performed in the US, and concerns over sudden unexpected death in epilepsy (SUDEP) in control arms prevents any non-treatment/wait-list designs.

In most of the studies it was not described whether AED treatment was reduced or discontinued and in those reporting on it, AED intake remained identical as prior to RT. Only two studies showed no improvement of seizure control after RT. ${ }^{28,30}$ A reason for this may be that RT is able to reduce the seizure frequency, but a vast number of the reported patients were not completely seizure free after RT thus requesting surgery. Furthermore, the long-term toxicity (e.g., cystic lesions) of RT may be another reason for the high rate of surgical interventions after primary RT. Interestingly, two studies investigated the cost effectiveness and found that surgery and Gamma Knife for hippocampal sclerosis were both safe and effective, but the surgical resection led to better results in freedom of epilepsy, (93\% versus $54 \%$ ) and a reduction of cost. ${ }^{49,50}$

\section{Limitations of the study}

Our study has some limitations. First of all, the only randomized study was retrievable in abstract-form only and attempts receiving a manuscript in preparation from the authors failed. Consequently, the work has not been peer-reviewed and thereupon modified yet, which hampers the resulting strength of our work. Although we checked in detail with available data, there is always the potential of patient overlap to occur between multicenter studies. In addition, control groups were not reported in the majority of studies, thereby limiting the strength of the study's design. Furthermore, studies described the primary outcome parameter differently using ILAE and pure description versus the Engel classification. Therefore the two reviewers (EP and DE) translated the seizure outcome of the corresponding studies to the RAEC to be able to compare the different studies on outcome. In the published studies the former ILAE classification on seizures and on epilepsies was used. Recently Fisher ${ }^{5}$ published the new ILAE classification renaming and specifying types of focal seizures and a few new generalized seizures, based on earlier clarification of terms used to name seizures. ${ }^{6}$ For this review, we assumed that the new term "focal epilepsy" is equivalent to the former term "localization related epilepsy".

Moreover, only 1 out of 16 studies reported information on the patient's quality of life. It is important that future research will include quality of life questionnaires in the design. Seizure outcome and long-term toxicity should be weighed against quality of life. In addition, two studies did not define their follow-up time. Nevertheless, we have 
decided to include these studies since important information about the efficacy of RT in epileptic patients was described ${ }^{27,28}$.

No conclusions can be drawn on the effect of fractionation compared to single-fraction on treatment outcome and toxicity due to limited amount of available fractionated studies.

In our dose response analyses we assumed the Linear-Quadratic (LQ) model to be correct in determining the $2 \mathrm{~Gy}$-per-fraction equivalent dose $\left(\mathrm{BED}_{10}\right)$ for fraction doses up to $24 \mathrm{~Gy}$. Brown et al. ${ }^{51}$ concludes that the LQ model is reasonably predictive of in vitro and in vivo normal tissue dose response relations in the dose per fraction range of 1.8 to $20 \mathrm{~Gy}$ and that it is currently not possible to identify an alternative high-dose model that performs better than the LQ for predicting cell killing. Even the lowest dose schedule showed a moderate result defined as Engel class I-III of 57\%. ${ }^{33}$ Side-effects were similar in this study except for cyst formation, which was not seen. In order to derive a possible future fractionation schedule, we derived a dose-effect relationship, however, the definition of the (correct) target volume based on reported outcome (epilepsy frequency) is mandatory though still lacking. We agree with Régis et al. ${ }^{52}$ suggesting that further basic research is needed for better understanding the influence of dose, volume, target topography and dose distribution homogeneity on the molecular effect in treating epilepsy. In addition, some recent studies hypothesize that the effect of RT should not be qualified as a destruction of the glial environment but as a modulation of the neural tissue. In particular in FNNE, this would be of interest ${ }^{12,20,23}$.

\section{Radiobiology}

The majority of treatments for epilepsy have been with SRS due to technical factors, mainly related to the requirements for immobilization of the patient's head and the availability of targeting systems. Although SRS has been shown to reduce seizure frequency in various forms of epilepsies, its mechanism of action remains unclear. Different mechanisms have been postulated as the basis of an anti-epileptic effect of SRS, such as the destruction of the epileptic focus and its pathways of spread by necrotizing SRS doses, or suppression of the epileptic activity as a neuromodulation effect at non-necrotizing doses ${ }^{53,54}$. There is no knowledge about fractionation effects for control of non-lesional epilepsy. From a radiobiological point of view, it is not clear whether SRS can better achieve the therapeutic goal than fractionated SRT with lower doses per fraction. Traditionally, the therapeutic effect of SRS is to destroy a small volume of brain tissue by radionecrosis, which results from vascular endothelial cell damage causing occlusion of small arterial vessels. There is growing interest that the same therapeutic effect may also be achieved by lower, fractionated doses of radiation, 
not inducing necrosis but having a neuromodulatory effect with possibly the same therapeutic and less unwanted effects. Studies are underway to investigate the threshold doses to achieve such neuromodulation ${ }^{53,55}$. Since there are no conclusive data on the value of the $\alpha / \beta$ of epileptogenic lesions, we assumed the $\alpha / \beta$ to be the same as for proliferating tissue like in brain metastasis or tumor tissue. It remains unknown whether the use of the $\alpha / \beta$ value of non-neoplastic tissue or even normal brain tissue would be more adequate. Although changing the value of $\alpha / \beta$ will change the absolute BED values, it hardly changes the relative positions of the data points in the dose response curve. Furthermore, this does not change the conclusion that the response correlates with BED. However, the difference in BED between single and multi-fraction schedules will increase: an $\alpha / \beta$ of 2.2 Gy instead of $10 \mathrm{~Gy}$ will cause a shift of the data points from the single-fraction schedules further to the right in relation to the multi-fraction schedules. This suggests the response is sensitive to fractionation, and a fractionated scheme would be favorable over a single-fraction scheme.

\section{Suggestions for future research}

This systematic review gives rise to the following suggestions for future research on RT for FNNE. First, more information on the quality of life of epileptic patients is compulsory (with information about AED reduction after treatment). Although seizures are not completely controlled after treatment, patients may benefit from seizure reduction, which is sometimes already a relief. Second, neurocognitive testing should be standard in studies on seizure frequency to gain knowledge on the effect of RT on the neurocognitive functioning. Thirdly, the definition of the (correct) target volume based on reported outcome (epilepsy frequency) is mandatory though still lacking. Functional MRI may augment defining this target volume. Fourth, little information is known about LINAC-based fractionated (stereotactic) radiotherapy and only four out of 16 studies that were included in this review used this technique. Hence, the effect of fractionation could not be assessed in detail. Therefore, the next step in assessing radiotherapy for FNNE is to improve the therapeutic ratio by reducing toxicity, which currently has a high incidence and is often the cause of resection after several years of follow-up. Bearing in mind the superior normal-tissue tolerance when employing a fractionated SRT scheme, future clinical trials may for example use 32-38 fractions of $1.8 \mathrm{~Gy}$ to obtain such response frequencies. The radiation techniques currently available allow for accurate dose delivery to a small volume of brain tissue. The first step to improve the therapeutic ratio would be to use lower total doses to similar volumes (e.g., by fractionation) in order to avoid healthy tissue destruction under isoeffect conditions. Once such doses have been shown to be equally effective, the 
radiosurgical use of charged particle beams with protons or carbon ions may further enhance the therapeutic ratio, based on their lower integral dose, lower risk of side effects and enhanced biological effectiveness. ${ }^{54-56}$ After improvement of the therapeutic ratio, prospective randomized-controlled trials to define the value of radiotherapy compared to anti-epileptic drugs, surgery, and other invasive approaches are urgently required.

\section{Conclusions}

Radiotherapy is a possible treatment option for focal epilepsy even though its present role in the management of drug-resistant epilepsy is limited. So far, no valid studies have been published to assess the efficacy of radiotherapy for drug-resistant FNNE in adults with a sufficiently high level of evidence. Since randomized control studies are lacking, existing evidence is mainly limited to case series. Hence, there is an urgent need for prospective randomized-controlled trials to define its value compared to antiepileptic drugs, surgery, and other invasive approaches. Further research is needed to establish agreement on target volume definition and to determine the optimal dose prescription in order to improve the current results of radiotherapy. 


\section{References}

1. Banerjee PN, Filippi D, Allen Hauser W. The descriptive epidemiology of epilepsy-a review. Epilepsy Res. 2009;85:31-45.

2. Rocha L. Interaction between electrical modulation of the brain and pharmacotherapy to control pharmacoresistant epilepsy. Pharmacol Ther 2013;138:211-28.

3. World Health Organization. The World Health Report 2001: Mental Health, New Understanding New Hope. Geneva: World Health Organization 2001;178.

4. Ngugi AK, Bottomley C, Kleinschmidt I et al. Estimation of the burden of active and life-time epilepsy: a meta-analytic approach. Epilepsia 2010;51:883-90.

5. Fisher RS. The New Classification of Seizures by the International League Against Epilepsy 2017. Curr Neurol Neurosci Rep. 2017;17(6):48.

6. Berg AT, Berkovic SF, Brodie MJ et al. Revised terminology and concepts for organization of seizures and epilepsies: report of the ILAE Commission on Classification and Terminology, 2005-2009. Epilepsia 2010;51:676-85.

7. Blumcke I, Spreafico R, Haaker G, Coras R, Kobow K et al. Histopathological Findings in Brain Tissue Obtained during Epilepsy Surgery. EEBB Consortium. New Engl J Med 2017;377(17):1648-16.

8. Moazzam AA, Wagle N, Shiroishi MS et al. Malignant transformation of DNETs: a case report and literature review. Neuroreport 2014;25(12):894-9.

9. Alexander H, Tannenburg A, Walker DG, Coyne T. Progressive dysembryoplastic neuroepithelial tumour. J Clin Neurosci. J Clin Neurosci 2015;22(1):221-4.

10. Riesberg G, Bathla G, Gupta S, Watal P, Moritani T. Malignant transformation and leptomeningeal spread of recurrent ganglioglioma: case report and review of literature. Clin Imaging 2017;48:7-11.

11. Régis J, Rey M, Bartolomei F, Vladyka V et al. Gamma knife surgery in mesial temporal lobe epilepsy: a prospective multicenter study. Epilepsia 2004;45:504-15.

12. Barbaro N, Quigg M, Broshek D et al. A multicenter, prospective pilot study of gamma knife radiosurgery for mesial temporal lobe epilepsy: seizure response, adverse events, and verbal memory. Ann Neurol 2009;65:167-75.

13. Régis J, Bartolomei F, Rey M et al. Gamma knife surgery for mesial temporal lobe epilepsy. J Neurosurg 2000;93:141-46.

14. Bartolomei $F$, Hayashi $M$, Tamura $M$ et al. Long-term efficacy of gamma knife radiosurgery in mesial temporal lobe epilepsy. Neurology 2008;70:1658-63.

15. Chang EF, Quigg M, Oh MC, Dillon WP, Ward MM, Laxer KD, Broshek DK, Barbaro NM; Epilepsy Radiosurgery Study Group. Predictors of efficacy after stereotactic radiosurgery for medial temporal lobe epilepsy. Neurology. 2010 12;74(2):165-72.

16. Boström JP, Delev D, Quesada C et al. Low-dose radiosurgery or hypofractionated stereotactic radiotherapy as treatment option in refractory epilepsy due to epileptogenic lesions in eloquent areas Preliminary report of feasibility and safety. Seizure 2016;36:57-62.

17. Wu C, Sperling MR, Falowski SM, Chitale AV, Werner-Wasik M et al. Radiosurgery for the treatment of dominant hemisphere periventricular heterotopia and intractable epilepsy in a series of three patients. Epilepsy Behav Case Rep 2012;1:1-6.

18. Choi H, Sell R, Lenert L, Muennig P et al. Epilepsy surgery for pharmacoresistant temporal lobe epilepsy: a decision analysis. JAMA 2008;300:497-505.

19. Uijl SG, Moons KG, Leijten FS et al. Is epilepsy surgery utilized to its full extent? Epilepsia 2008;49: 1480-1.

20. Régis J. Gamma knife for functional diseases. Neurotherapeutics 2014;11:583-92.

21. Quigg $M$, Harden $C$. Minimally invasive techniques for epilepsy surgery: stereotactic radiosurgery and other technologies. J Neurosurg 2014;121:232-40.

22. Kim YJ, Cho KH, Kim JY et al. Single-dose versus fractionated stereotactic radiotherapy for brain metastases. Int J Radiat Oncol Biol Phys 2011;81:483-9.

23. Régis J, Carron R, Park M. Is radiosurgery a neuromodulation therapy? A 2009 fabrikant award lecture. J Neurooncol 2010;98:155-62. 
24. Engel Jr J, Van Ness P, Rasmussen T. Outcome with respect to epileptic seizures. Surgical treatment of the epilepsies. Raven Press (in press 1993).

25. Liberati A, Altman D, Tetzlaff J. The PRISMA statement for reporting systematic reviews and metaanalyses of studies that evaluate health care interventions: explanation and elaboration. J Clin Epidemiol 2009;62.

26. Rheims S, Fischer C, Ryvlin P et al. Long-term outcome of gamma-knife surgery in temporal lobe epilepsy. Epilepsy Res 2008;80:23-9.

27. Usami K, Kawai K, Koga T et al. Delayed complication after Gamma Knife surgery for mesial temporal lobe epilepsy. J Neurosurg 2012;116:1221-5.

28. Kawai K, Suzuki I, Kurita $\mathrm{H}$ et al. Failure of low-dose radiosurgery to control temporal lobe epilepsyreport of two cases. J Neurosurg 2001;95:883-7.

29. Kawamura $T$, Onishi $H$, Kohda $Y$ et al. Serious adverse effects of gamma knife radiosurgery for mesial temporal lobe epilepsy. Neurol Med Chir 2012;52:892-8.

30. Srikijvilaikul T, Najm I, Foldvary-Schaefer $\mathrm{N}$ et al. Failure of gamma knife radiosurgery for mesial temporal lobe epilepsy: report of five cases. Neurosurgery 2004;54:1395-402.

31. Rauch C, Semrau S, Fietkau R et al. Long-term experience with fractionated stereotactic radiotherapy in pharmacoresistant epilepsy: neurological and MRI changes. Epilepsy Res 2012;99:14-20.

32. Grabenbauer G, Reinhold C, Kerling F et al. Fractionated stereotactically guided radiotherapy of pharmacoresistant temporal lobe epilepsy. Acta Neurochir Suppl 2002;84:65-70.

33. Liang S, Liu T, Li A et al. Long-term follow up of very low-dose LINAC based stereotactic radiotherapy in temporal lobe epilepsy. Epilepsy Res 2010;90:60-7.

34. Hoggard N, Wilkinson I, Griffiths $P$ et al. The clinical course after stereotactic radiosurgical amygdalohippocampectomy with neuroradiological correlates. Neurosurgery 2008;62:336-44.

35. Chen N, Du S, Yan N et al. Delayed complications after Gamma Knife surgery for intractable epilepsy. J Clin Neurosci 2014;21:1525-8.

36. Vojtech Z, Vladyka V, Kalina $\mathrm{M}$ et al. The use of radiosurgery for the treatment of mesial temporal lobe epilepsy and long-term results. Epilepsia 2009;50:2061-71.

37. Quigg M1, Broshek DK, Barbaro NM, Ward MM, Laxer KD, Yan G, Lamborn K; Radiosurgery Epilepsy Study Group. Neuropsychological outcomes after Gamma Knife radiosurgery for mesial temporal lobe epilepsy: a prospective multicenter study. Epilepsia. 2011;52(5):909-16.

38. Vojtěch Z, Krámská L, Malíková H, Stará M, Liščák R. Neuropsychological results after gamma knife radiosurgery for mesial temporal lobe epilepsy. Neuro Endocrinol Lett. 2015;36(8):771-8.

39. Taphoorn MJ. Neurocognitive sequelae in the treatment of low-grade gliomas. Semin Oncol 2003;30: 45-8.

40. Kurita H, Suzuki I, Shin M, Kawai K, Tago M, Momose T, Kirino T. Successful radiosurgical treatment of lesional epilepsy of mesial temporal origin. Minim Invasive Neurosurg. 2001;44(1):43-6.

41. Irislimane M, Mathieu D, Bouthillier A, Deacon C, Nguyen DK. Gamma knife surgery for refractory insular cortex epilepsy. Stereotact Funct Neurosurg. 2013;91(3):170-6.

42. Yen DJ, Chung WY, Shih YH, Chen C, Lirng JF, Yiu CH, Yu HY, Su TP, Pan DH. Gamma knife radiosurgery for the treatment of recurrent seizures after incomplete anterior temporal lobectomy. Seizure. 2009;18(7):511-4.

43. Shim KW, Chang JH, Park YG, Kim HD, Choi JU, Kim DS. Treatment modality for intractable epilepsy in hypothalamic hamartomatous lesions. Neurosurgery. 2008;62(4):847-56.

44. Maesawa S, Kondziolka D, Dixon CE et al. Subnecrotic stereotactic radiosurgery controlling epilepsy produced by kainic acid injection in rats. J Neurosurg 2000;93:1033-40.

45. Ladino LD, Dash C, Wu A, Tellez-Zenteno JF. Intracranial investigation of a patient with nodular heterotopia and hippocampal sclerosis: dealing with a dual pathology. Epileptic Disord. 2017;19(2): 195-201.

46. Stefan H, Nimsky C, Scheler G, Rampp S, Hopfengärtner R, Hammen T, Dörfler A, Blümcke I, Romstöck J. Periventricular nodular heterotopia: A challenge for epilepsy surgery. Seizure. 2007;16(1):81-6.

47. Howick J, Chalmers I, Glasziou P et al. OCEBM Table of Levels of Evidence Working Group. "The Oxford 2011 Levels of Evidence". Oxford Centre for Evidence-Based Medicine. http://www.cebm.net/ index.aspx?o=5653. 
48. Wiebe S, Blume WT, Girvin JP, Eliasziw M; Effectiveness and Efficiency of Surgery for Temporal Lobe Epilepsy Study Group. A randomized, controlled trial of surgery for temporal-lobe epilepsy. N Engl J Med. 2001;2;345(5):311-8.

49. Han ZT, Chen QX. Curative effect and costs of surgical and gamma knife treatments on intractable epilepsy caused by temporal-hippocampal sclerosis. Genet Mol Res 2015;14:8555-62.

50. Xiao A, Wang T, Tian Y et al. Clinical effects of surgical and Gamma Knife treatments on hippocampal sclerosis-induced intractable epilepsy of children below age 10 years. Pak J Med Sci 2013;29:943-6.

51. Brown JM, Carlson DJ, Brenner DJ. The tumor radiobiology of SRS and SBRT: Are more than the 5 Rs involved? Int J Radiat Oncol Biol Phys. 2014;88(2):254-62.

52. Régis J, Kerkerian-Legoff L, Rey $M$ et al. First biochemical evidence of differential functional effects following Gamma Knife surgery. Stereotact Funct Neurosurg 1996;66:29-38.

53. Quigg M, Rolston J, Barbaro NM. Radiosurgery for epilepsy 2012: clinical experience and potential antiepileptic mechanisms. Epilepsia 2012;53:7-15.

54. Madani I, Lomax AJ, Albertini F et al. Dose-painting intensity-modulated proton therapy for intermediate- and high-risk meningioma. Radiat Oncol 2015;10:72.

55. Prezado Y, Fois GR. Proton-minibeam radiation therapy: a proof of concept. Med Phys 2013;40:031712.

56. Studer F, Serduc R, Pouyatos B, et al. Synchrotron X-ray microbeams: A promising tool for drug-resistant epilepsy treatment. Phys Med 2015;31:607-14. 


\section{Supplemental material}

Table S7.1 Medical subjects headings per database.

\begin{tabular}{ll}
\hline Database & Medical Subject Headings (MeSH terms) \\
\hline Pubmed & $\begin{array}{l}\text { epilepsy AND radiotherapy AND seizures AND } \\
\text { control groups AND radiotherapy dosage AND } \\
\text { maximum tolerated dose AND radiation effects } \\
\text { Ovid Medline }\end{array}$ \\
$\begin{array}{l}\text { The Cochraine Library } \\
\text { Embase }\end{array}$ & $\begin{array}{l}\text { radiotherapy AND epilepsy AND seizure control } \\
\text { rand epilepsy AND seizure control }\end{array}$ \\
Web of Science & AND seizure control \\
& radiotherapy AND radiation AND epilepsy \\
& AND seizure control \\
\hline
\end{tabular}

Table S7.2 Search history PubMed.

\begin{tabular}{l}
\hline Search items \\
\hline (("epilepsy"[MeSH Terms] OR "epilepsy"[All Fields]) AND ("radiotherapy"[Subheading] OR "radiotherapy"[All \\
Fields] OR "radiotherapy"[MeSH Terms])) AND (Outcome[All Fields] OR (("seizures"[MeSH Terms] OR \\
"seizures"[All Fields] OR "seizure"[All Fields]) AND ("prevention and control"[Subheading] OR \\
("prevention"[All Fields] AND "control"[All Fields]) OR "prevention and control"[All Fields] OR "control"[All \\
Fields] OR "control groups"[MeSH Terms] OR ("control"[All Fields] AND "groups"[All Fields]) OR "control \\
groups"[All Fields])))) AND (("radiotherapy dosage"[MeSH Terms] OR ("radiotherapy"[All Fields] AND \\
"dosage"[All Fields]) OR "radiotherapy dosage"[All Fields]) OR ("maximum tolerated dose"[MeSH Terms] OR \\
("maximum"[All Fields] AND "tolerated"[All Fields] AND "dose"[All Fields]) OR "maximum tolerated dose"[All \\
Fields]) OR ("radiation effects"[Subheading] OR ("radiation"[All Fields] AND "effects"[All Fields]) OR "radiation \\
effects"[All Fields] OR "radiation effects"[MeSH Terms] OR ("radiation"[All Fields] AND "effects"[All Fields]))) \\
\hline
\end{tabular}

Table S7.3 In and exclusion criteria.

\begin{tabular}{|c|c|}
\hline Inclusion criteria & Exclusion criteria \\
\hline V Studies published after 1990 & V Animal studies \\
\hline V English-written & $\checkmark$ Pediatric patient population ( $<18$ years of age) \\
\hline \multirow{2}{*}{$\begin{array}{l}\text { V Minimum follow-up time of } 12 \text { months after } \\
\text { radiotherapy available }\end{array}$} & $\checkmark$ Case reports \\
\hline & $\checkmark$ Prior resection \\
\hline V Seizure frequency reduction outcome available & V Possible neoplastic causes of epilepsy \\
\hline $\begin{array}{l}\text { V Patients older than } 18 \text { years with focal drug- } \\
\text { resistant epilepsy }\end{array}$ & $\begin{array}{l}\text { (including arteriovenous malformations, } \\
\text { hamartoma, cavernoma and neoplasms) }\end{array}$ \\
\hline $\begin{array}{l}V \text { Information on medication reduction available and } \\
\text { information on dose prescription }\end{array}$ & $\begin{array}{l}\text { V Bias caused by patient selection linked to outcome } \\
\text { or toxicity }\end{array}$ \\
\hline \multirow{2}{*}{$\begin{array}{l}V \text { Treatment technique and toxicity of radiotherapy } \\
\text { available }\end{array}$} & $\checkmark$ Non-English \\
\hline & $\begin{array}{l}\text { V Articles that were not accessible and could not be } \\
\text { provided by the corresponding author }\end{array}$ \\
\hline
\end{tabular}


Focal epilepsy treated with stereotactic radiotherapy

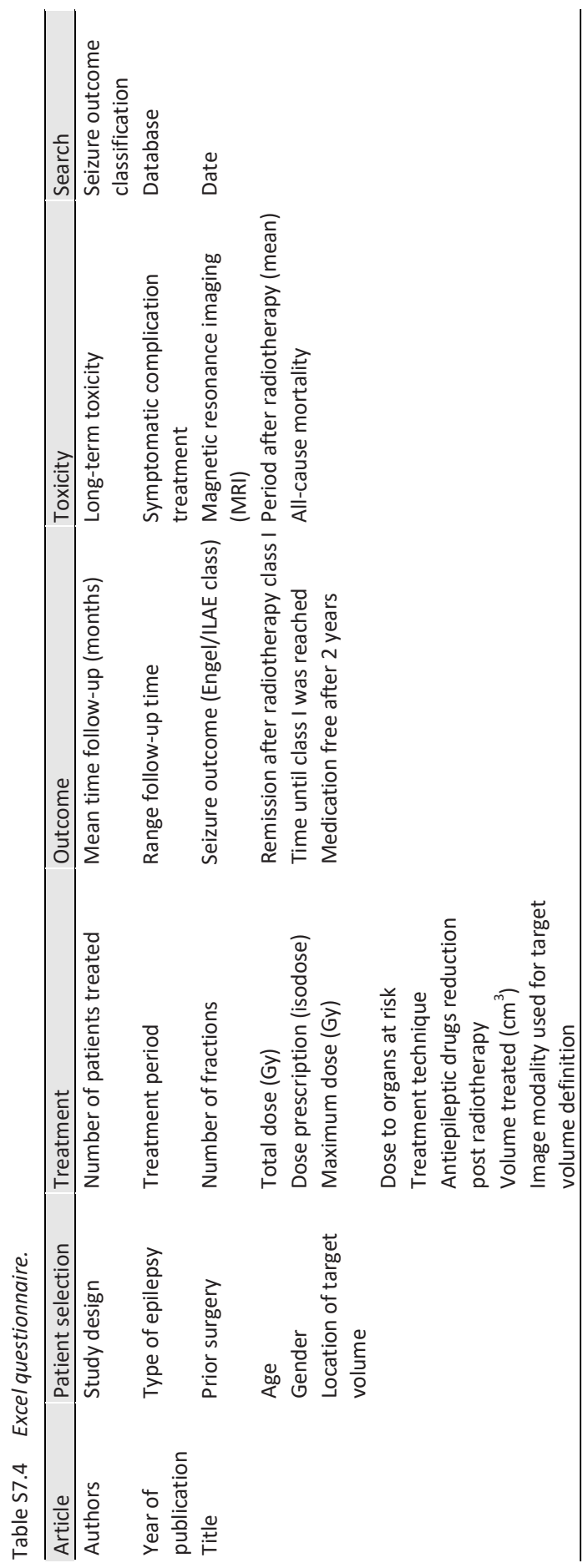


Chapter 7

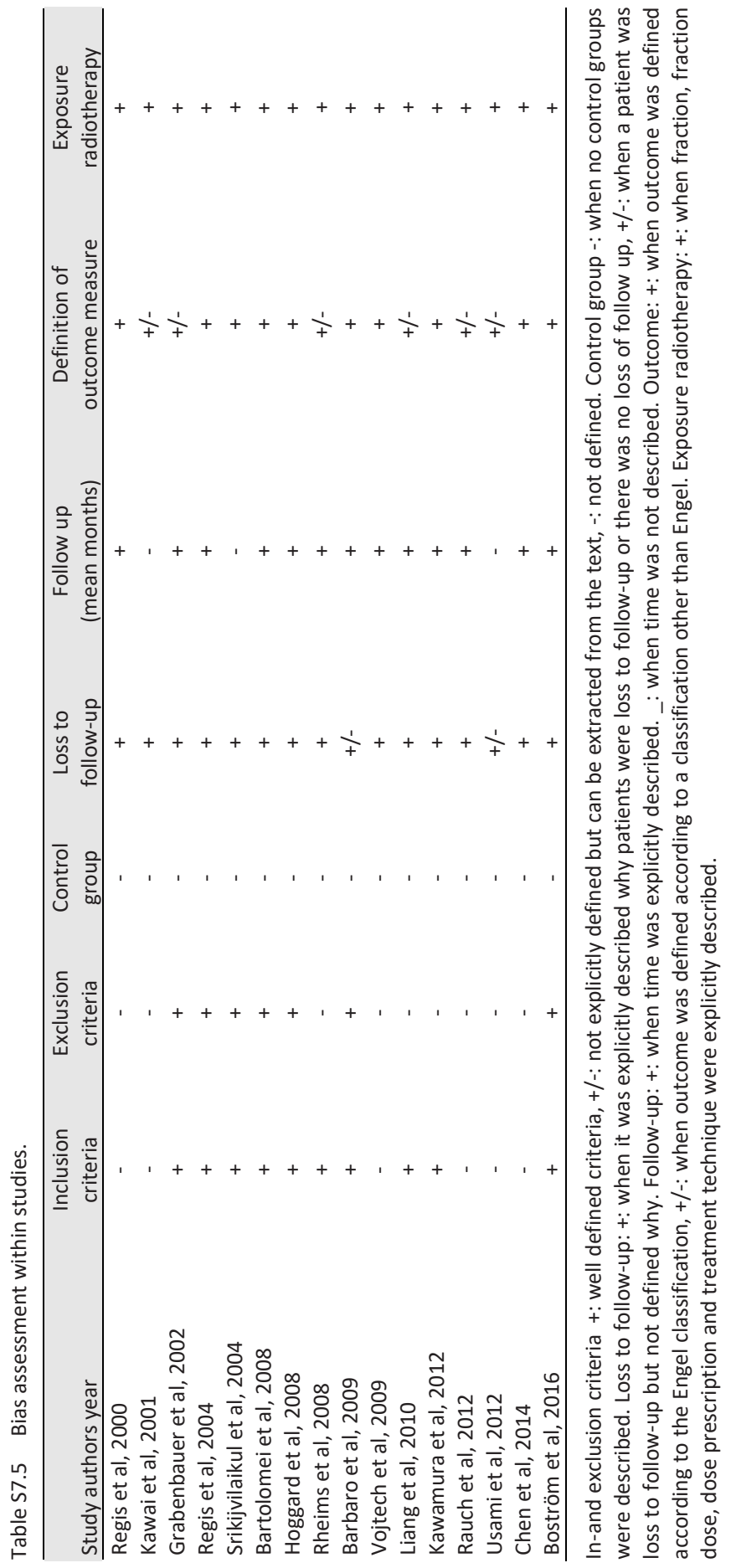




\section{Chapter 8}

General discussion 



\section{Discussion}

The overall aim of this thesis was to optimize radiotherapy of primary tumors of the brain and head-and-neck, specifically by assessing the therapeutic value of particle therapy (PT).

When treating a tumor with radiotherapy, a reasonable balance is needed between tumor control probability (TCP) and normal tissue complication probability (NTCP). In this thesis, we mainly investigated potential improvements in NTCP models. Since these NTCP models already existed for the primary treatment of HNSCC, I particularly focused on tumors and organs at risk (OARs) of the central nervous system (CNS) ${ }^{1-4}$. This included determining the clinically relevant OARs of the CNS, uniforming their delineation by introducing a CNS atlas, and reporting on a consensus on tolerance dose. These elements are indispensable for the development of NTCP models needed for an optimal plan comparison between different treatment modalities such as photon and particle therapy. This is especially true when costs or availability limit one of the treatment options, as is the case in the Netherlands were only $3 \%$ of all radiotherapy patients are eligible for proton therapy within the coming years.

Due to the limited resources and in order to accelerate the generation of clinical evidence, the Dutch model based approach was introduced. This approach consists of 4 steps $^{5,6}$. The first step is the selection of validated, photon-based NTCP models from literature by experts in the field. The second step is the prediction of the individual patient's NTCP values for a certain toxicity taking the baseline toxicity into account. When a certain threshold is exceeded, a plan comparison between photon and proton therapy is indicated. Step 3 is individual patient selection by determining the delta NTCP of the two treatment plans, indicating the predicted gain by reducing toxicity. Within the Netherlands there is an agreement to use the common terminology criteria for adverse events version 4 (CTCAE-4). Since CTCAE-4 grade 1 toxicity gives no permanent damage, only grade 2 to 4 are taken into account. Grade 2, 3 and 4 toxicities require a delta NTCP of $10 \%, 5 \%$ and $2 \%$, respectively, to favor the treatment with proton beam therapy. The fourth step is the validation or modification of the existing, photon-based NTCP models, on the basis of the observed toxicity following proton beam therapy ${ }^{5,6}$.

In the current absence of validated NTCP models for CNS and HNSCC re-irradiation, a dosimetric plan comparison was conducted in two ROCOCO trials. These data can assist in decision-making which treatment modality is expected to be the most favorable for an individual patient in reducing the side effects caused by radiotherapy. However, the 
development of these desired NTCP models itself was no subject of this thesis and this needs to be investigated in future research. These NTCP models will not only focus on dose-response relationships for treatment related toxicity, but ideally these will also take patient characteristics and other treatment modalities such as systemic treatments into account. By this the NTCP models could assist in developing decision support systems and shared decision tools, which enable not only the doctor but also the patient to make a well balanced choice between surgery, systemic therapy or various options of radiotherapy ${ }^{7-11}$. This principle also applies for nonmalignant diseases, for example epilepsy. Currently, there is growing interest in treating this disease with high-conformal radiation techniques, e.g., proton beams. Until now it is unknown what dose is required to treat foci of epilepsy. Therefore, there is a need for a radiation dose response model in epilepsy. For a validated dose response model future clinical trials are needed to validate the data retrieved from retrospective studies.

\section{Plan comparison HNSCC \& CNS}

Through comparison of state of the art treatment plans in the ROCOCO trial for re-RT of HNSCC and low grade glioma (LGG) we demonstrated that particle therapy (PT) improves the sparing of most OARs, with evident reduction of the integral dose ${ }^{12,13}$. Carbon ions were superior to protons in most cases in the ROCOCO re-RT HNSCC trial, which can be explained by the different beam characteristics such as a sharper penumbra of carbon ions as also demonstrated in several other planning studies ${ }^{14,15}$. Unfortunately, no randomised trials have yet been performed confirming a superior clinical benefit for carbon ions above protons in reducing the dose to the OARs ${ }^{12}$. This is likely due to limited access and increased costs of any of the two modalities compared to photon-based treatment. Tomotherapy (TOMO) was found to be superior to volumetric modulated arc therapy (VMAT) in sparing OARs in the ROCOCO LGG trial, most striking the centrally located OARs such as the pituitary gland ${ }^{13}$. This is in agreement with Koca et $a{ }^{16}{ }^{16}$ showing TOMO to be superior to intensity-modulated radiotherapy (IMRT) in sparing OAR in a plan comparison of 20 glioblastoma patients.

A high dose conformity index to the clinical target volume was achieved by using VMAT in the LGG trial, followed by TOMO and IMRT ${ }^{13}$. This lower conformity index of particle therapy was most likely caused by the necessity of robust treatment planning in particle therapy. Resulting in a good coverage maintenance while no tolerance doses are exceeded. Despite a variety of setup changed during treatment. Creating an area of higher dose around the clinical target volume (CTV) exceeding slightly the planning target volume (PTV) used in photon planning such as VMAT, IMRT and TOMO. Particle therapy is more sensitive to density changes than photon therapy. Making robust 
optimization necessary to account for possible positioning errors. Causing a slightly different path length for the proton beam causing relevant dose differences.

Weight loss, mucous filling of the air-filled sinuses or tumor regression will also influence the dose distribution and require repeated imaging and recalculation of the treatment plan, most profound in HNSCC patients ${ }^{17-21}$. This leads to one of the limitations of this in silico study being the fact that density changes, caused by these anatomical changes during therapy, were not taken into account. This possibly influences the outcome and might in daily practice require regularly new computed tomography (CT) and treatment plan adaptation during the course of treatment. This may possible influence the NTCP for that particular OAR after calculating the cumulative dose distribution.

Another limitation not taken into account in the ROCOCO study even though possibly influencing the plan comparison is the presence of metal artefacts, such as dental fillings and crowns, within the particle beam. In our ROCOCO study these metal filling, including the streak artefacts on the planning-CT caused by them, were delineated and replaced by water densities since they were considered of small influence on the dosimetric outcome of this comparative trial. In a phantom study of Richards et al. ${ }^{22}$, the baseline proton dose ranges of $98-106 \%$ around the planning target volume (PTV) resulted in $66-111 \%$ coverage in the presence of metal fillings. In daily practice it is strongly advised to avoid metal in the line of the proton beam. If this cannot be avoided, it should be considered to remove, if possible, this metal ${ }^{23}$.

A third factor confounding the results is that in these ROCOCO studies treatment plans were generated in different institutes having, besides the study protocol, their own clinical practice and internal protocols. Therefore, the treatment planning procedures may not necessary be the same possibly causing an altered numerical outcome, for example, due to the slightly different PTV margins, determined by each participating department. Likewise, the influence of the beam angles and the number of beams cannot be excluded. In our ROCOCO trials we found that in particular the contralateral OARs benefit from particle therapy. There are some publications confirming this observation. The largest being the retrospective study from Harrabi et al. ${ }^{24}$ in which 74 LGG patients were treated with proton therapy. The delivered proton treatment plan were compared to generated non state of the art conventional three-dimensional radiotherapy plans showing comparable coverage of the target volume and reduction of the dose especially in the contralateral OARs.

After both ROCOCO trails it remains unclear if the reported reductions in dose to the OARs will result in a reduction of clinical relevant side effects. This will need to be proven in future research. This is in particular true for the ROCOCO LGG trial in which the cerebellum posterior is incorporated as a possible new CNS OAR ${ }^{26}$. 


\section{Organs at risk for treatment of CNS tumors}

\section{Determination}

Due to the use of multimodality therapy, the survival rates increase for LGG patients, raising awareness to the long-term side effects of which neurocognitive decline is one of the most feared ones due to its direct influence on the patient's quality of life ${ }^{26}$. Apart from the paper by Gondi et al. ${ }^{27}$, it is still unclear what OARs cause this cognitive decline and at which radiation dose. Gondi et al. ${ }^{27}$ described a NTCP model based on the dose to $40 \%$ of both hippocampi, which significantly correlated with cognitive decline in 18 patients. The cerebral hemispheres are considered responsible for cognitive function, whereas the cerebellum is known for its role in regulating and coordinating movement, balance and posture ${ }^{28}$. In this thesis, through a review of the available literature, the possible role of the cerebellum in cognition is described. Schmahmann et al. ${ }^{29}$ suggested the division of the cerebellum in an anterior and posterior part. Merchant et $a .^{30}$ was the first to delineate the anterior and posterior part separately according to this, in his pediatric LGG study. They evaluated 78 children with LGG treated with radiotherapy with or without prior chemotherapy. A significant relationship was found between neurocognitive impairment and the radiation dose to the posterior cerebellum ${ }^{30}$. Gan et al. ${ }^{31}$ studied 10 adult head and neck cancer patients treated with radiation therapy and showed that memory was the most severely affected cognitive domain. The one patient most affected received a maximum dose to the cerebellum of $36 \mathrm{~Gy}$ with a low dose to the whole brain and hippocampi. To date these data still need to be confirmed in a prospective study. In order to do so, agreement is needed in how to delineate the posterior and anterior part of the cerebellum ${ }^{32,33}$.

Besides previously described cognitive impairment, other relevant toxicity require delineation of other OARs e.g. for vision, hearing, endocrine and brainstem function. An uniform delineation of the related OARs such as optical nerve, chiasm, cochlea, pituitary and brainstem is mandatory.

\section{Delineation}

Reduction of the inter- and intra-observer variability for delineation of OARs (and target-volumes) in radiation oncology is greatly depending on the use of guidelines for delineation ${ }^{34}$. This guidelines can be offered by an atlas. In daily practice and even more indispensable in conducting clinical trials, the conformation to a region specific delineation atlas is crucial in comparing dose to the delineated structures. In head and neck cancer, Brouwer et al. ${ }^{35}$ composed a consensus guideline delineation atlas in 
cooperation with DAHANCA, EORTC, GORTEC, HKNPCSG, NCIC CTG, NCRI, NRG Oncology and TROG for this purpose. Also for thoracic radiotherapy an OAR atlas is available provided by Kong et al. ${ }^{36}$ In accordance, Vinod et al. ${ }^{37}$ concluded in their review of 56 studies, which evaluated delineation, that the inter-observer variability in delineation of the target volume and OARs can be reduced with the use of guidelines, provision of auto contours and teaching including the use of multimodality imaging, which was considered useful in certain tumor sites such as CNS. Conversely, a recent study of Bartel et al..$^{38}$ demonstrated a large inter-observer variation of hippocampus delineation in a hippocampus avoidance study. They evaluated the delineation of 10 hippocampi by 4 radiation oncologists, 2 radiation technicians and one neuroradiologist, all from different institutions, using the EORTC hippocampal contouring atlas by Gondi et al. ${ }^{39}$. Concluding that even with the use of an atlas the delineation variability remains large. Illustrating that the upload and approval of the OARs delineation should be part of the quality assurance procedures of clinical trials.

In order to create uniform CNS delineation, the first step was to agree upon which OARs are relevant for CNS radiation, excluding the specific head and neck OARs as defined in the Brouwer atlas ${ }^{34}$. OARs deemed relevant for radiation-induced toxicity in neuro oncology and to be included in the new CNS delineation atlas were: brain, brainstem, chiasm, cochlea, cornea, lacrimal gland, hippocampus, lens, optic nerve, pituitary, retina, skin and vestibulum \& semicircular canals. The second step was defining the borders of those specific OARs based on their anatomy on CT and magnetic resonance (MR) imaging. The third step was the development of an online available CT and MR based contouring atlas. The fourth and last crucial step to ensure wide spread use of the atlas was reaching a consensus on all previously mentioned steps within the European Particle Therapy Network (EPTN) ${ }^{32,33}$.

For efficient digital comparison of different radiation modalities a uniform nomenclature based on standardizing naming convention in radiation oncology by Santanam et $a l^{40}$ was used.

A potential limitation of this atlas is the limitation of OARs. Anterior eye chambers, cerebrospinal space, circle of Willis, Eustachian tube, frontal \& temporal lobe, optic tract, macula, mammillary bodies and spinal canal are possible relevant OARs for which future research might show its relevance. Even though, due to practicality and resources, the international panel of experts decided to adhere to the OARs in the current atlas and to extend the set if shown relevant in the future. Since the atlas is available online it will be updated when indicated for these or other future relevant $\mathrm{OARs}^{32}$. 


\section{Tolerance dose}

After uniform CNS delineation using a CT and MR based CNS atlas, a consensus on the dose constraints of these OARs is mandatory ${ }^{33}$. A comprehensive search of the current literature on the aforementioned OARs on dose constraints was performed ${ }^{41}$. For each of these OARs the available data were summarized and a dose constraint was determined in consensus with 20 Radiation Oncology experts in the field of neuro oncology within the EPTN. All dose constraints and corresponding $\alpha / \beta$ values were reported in Gy. The doses were recalculated to equivalent dose in 2 Gy-fractions (EQD2).

Damage to a serial OARs, e.g., the optic nerve, chiasm, cornea, retina, lens, skin and brainstem, will lead to toxicity after damage of only a sub-volume of this OAR. For these OARs a volume constraint was added as a near maximum value. Only for the serial OARs the tolerance dose versus the total number of fractions was recalculated based on the consensus constraints for serial OARs, using the linear quadratic formula ${ }^{41}$. These values were defined as near maximum EQD2 values.

Parallel OARs being cochlea, hippocampus, lacrimal gland and pituitary, will lose their function depending on the mean dose to the whole structure. For the parallel OARs no tolerance dose per fraction was calculated since according to Perko et al. ${ }^{43}$ and Hoffmann et $a l .{ }^{44}$ these are comprised of the treatment plan's dose and its standard deviation, which are patient- and technique- dependent.

A confounding factor of this study is that the consensus tolerance doses could only be based on the scarce information found in literature on the dose and toxicity relationship for most of the OARs described in the neuro delineation atlas. Some possible relevant OARs such as the vestibulum and semi-circular canals, hypothalamus and the cerebellum could not be included in this consensus document, because the required data are still lacking. In order to pursue consenting a dose, these data and an accurate delineation is most relevant in order to collect data and related toxicity in the future.

\section{Radiotherapy for non-neoplastic Epilepsy}

In the previous studies effort was made to assist in developing NTCP models in the central nervous system. Similar to an NTCP model predicting the possibility of toxicity, a TCP model predicts the possibility on treatment response, in oncology this is equivalent to tumor response. In a disease without a tumor, i.e., non-neoplastic epilepsy, there is also a need for a dose-response model.

In order to find evidence on the effectiveness of radiotherapy in drug-resistant nonneoplastic focal epilepsy in adults and to fit the dose-effect model, we conducted a 
systematic literature search. Sixteen studies out of 170 initially identified studies on drug-resistant non-neoplastic focal epilepsy in adults were included, including in total 170 patients ${ }^{45}$. A positive effect of radiotherapy on the seizure frequency was seen in 12 of the 16 studies, showing and average reduction in $58 \%$ of the patients, meaning no or rare seizures defined as radiotherapy-adapted Engel class (RAEC) I and II. Despite the positive effect of radiotherapy, $20 \%$ of the patients still needed surgery afterwards due to toxicity or remaining seizures. The toxicity requiring surgery included radionecrosis, cysts formation, edema and intracranial hypertension.

With the available data a dose-effect model was fitted between the prescribed dose and the seizure reduction score. This study showed that radiotherapy is a good alternative treatment in drug resistant non-neoplastic focal epilepsy in adults, although further research is needed to determine its role compared to other treatment modalities such as MR-focused ultrasound and laser interstitial thermal therapy, which were not subject of our study ${ }^{46}$.

The major shortcoming of that review is the fact that the Rose trial ${ }^{47}$ could not be incorporated since it was published after the publication of our review. In the Rose trial, stereotactic radiosurgery to a dose of $24 \mathrm{~Gy}$ was randomized to anterior temporal lobe lobectomy for patients with drug resistant unilateral temporal lobe epilepsy ${ }^{47}$. After 36 months, $74 \%$ of patients treated with stereotactic radiosurgery were seizure free versus $85 \%$ of the patient after an anterior temporal lobe resection. An important observation in the Rose trial confirmed within our 6 reviewed studies, is the time to response, being 3-6 months after surgery compared to 24 months after radiotherapy ${ }^{45,47}$. There was no significant difference in quality of life in epilepsy (QOLIE) between the two groups in the Rose trial confirming our conclusion that radiotherapy seems a good alternative to respective surgery in drug-resistant epilepsy.

\section{Future perspectives}

Particle therapy, such as proton therapy is not a new treatment. The first patient was treated in 1954 and subsequently over 60.000 patients have been treated worldwide ${ }^{48}$. Still, solid evidence of equivalence or superiority of particle therapy is lacking. Level I evidence would include a randomized trial, comparing particle therapy to state of the art photon radiotherapy. In the last decade, it has been proven difficult to perform such a randomized trial. Costs are a crucial factor, since health insurance companies are reluctant to reimburse a treatment without convincing evidence, making this lack of evidence-discussion a vicious circle. Even if a randomized trial is conducted, e.g. the LGG trial (clinicaltrial.gov NCT03180502) open for inclusion since 2017 with cognition being the primary endpoint, it will take years before results become available and 
radiation treatment techniques will have developed, still raising doubt on the clinical results and thus leaving us one step behind.

For eye melanoma, chordoma and chondrosarcoma, there is mature evidence of high local control rates and low toxicity, which is very difficult to achieve with photon therapy due to the required high dose and the proximity of the target to critical OARs. For paediatric tumours, there is a broad acceptance of particle therapy by the general public and health insurance companies due to the striking reduction of the integral dose, which can be achieved using particle therapy, resulting in a putative reduction of secondary tumour induction. As Schneider et $a . .^{50}$ stated in his review, radiation induced secondary cancers can be the price of success if the primary cancer is cured or at least controlled.

As of early 2018 particle therapy has become available for daily patient care in The Netherlands. It is expected that within the next 4-6 years up to $3 \%$ of all patients referred for radiotherapy can be treated with proton therapy. Since resources are scarce and costs high (roughly estimated to be 3 times higher compared to photon therapy), a good patient selection is therefore warranted.

Thanks to the impressive national collaboration between all radiotherapy department throughout The Netherlands, a solid model based approach was adopted, enabling the doctors to select only the right patient for proton therapy ${ }^{5}$. In this model a plan comparison is required for the non-standard indications. Standard indication are paediatric tumours, chordoma \& chondrosarcoma and eye tumours. Recently, craniospinal axis irradiation and primary brain tumours with a 10 year overall survival expectancy of more than 50\%, excluding all brain tumours eligible for stereotactic radiotherapy, were added to the standard indications.

The health insurance companies agreed to this approach, under the condition that within the next decade data are provided supporting the hypothesis that in a selected patient group, proton therapy is superior to photon therapy. Only if good patient selection is performed and all Dutch radiotherapy departments work closely together, The Netherlands might be able to show the gain of proton therapy compared to photon therapy. This principal is essentially different from earlier attempts to introduce particle therapy. Some particle centres even had to face bankruptcy in the past. Some causative factors being the huge investments, patient referral lagging behind and non-substantial reimbursement. Leading to the increasing need to accept patients able to pay for the treatment themselves and not necessary the most likely to benefit from particle therapy.

In this thesis we showed that particle therapy has dosimetric advantages over photon therapy in HNSCC re-irradiation and LGG, but there is still a need to confirm these in 
silico treatment planning studies with real clinical data (see Figure 8.1). In order to prevent large costly trials, upfront patient selection is needed and for that validated NTCP models are mandatory. This is not only needed for proton therapy but it will also be of great value in photon treatment. Accurate NTCP models enable the radiation oncologist to predict for each and every patient as precisely as possible the change of a certain toxicity, giving certain patient characteristics. In order to achieve this goal future research is needed, especially in patient specific factors. Whether these factors arise from genetic profiles, imaging (radiomics), blood samples, co-morbidity or are as simple as age or gender, a lot of work still has to be done for each OAR.

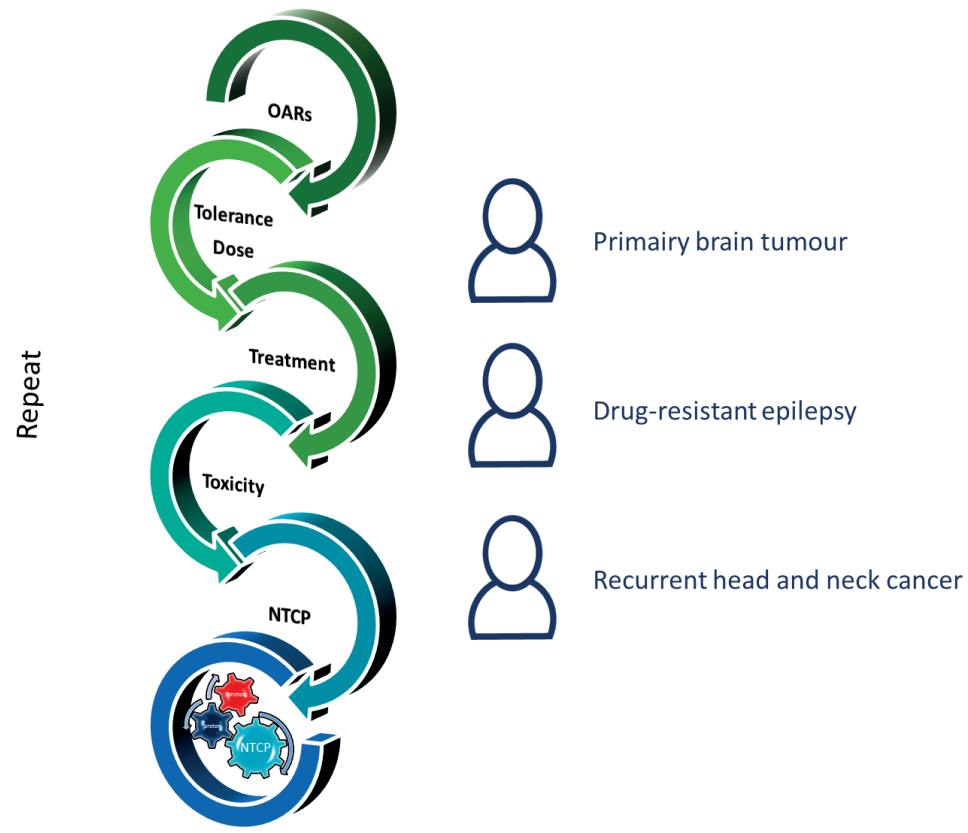

Figure 8.1 Repetitive cycle subject of this thesis optimizing brain and head \& neck radiotherapy illustrating the need for clinical data. NTCP = normal tissue complication probability model. Wheel shapes red $=$ proton radiotherapy, dark blue $=$ photon radiotherapy, light blue $=$ NTCP model .

Since the amount of data required due to all different variables, good cooperation with the particle centres, as well as all photon facilities is needed resulting eventually in a shared goal; the best treatment available for our patients. The EPTN is such an initiative in which radiation oncologist from particle centres all over Europe work together harmonise, gather and analyse data. 
In this thesis a consensus within the CNS experts of the EPTN was reached in order to facilitate combining data in the future by agreeing to delineate OARs in a uniform way and take the same tolerance does into account during treatment planning. Even if there is only limited evidence for a certain tolerance dose, a uniform threshold enables us to collect data to accept or reject the threshold in time. Due to increasing interest in particle therapy and evolving technology, the size and costs of a cyclotron keeps declining resulting in smaller proton facilities most of them combined with photon radiotherapy, making this treatment in future more accessible to all patients in Europe. As a result of these smaller proton facilities, it becomes even more essential to work together to get the data needed to improve patient selection by improving NTCP models including molecular markers and imaging. Elaborating a cooperation such as the ROCOCO initiative in with international parties work together. This underlines the need for rapid collecting/pooling and exchanging of international research data in the field of radiotherapy as suggested by Skripcak et $a{ }^{4}{ }^{49}$. They describe the challenges that have to be addressed such as utilisation of standards, data quality and privacy concerns, data ownership, rights to publish, data pooling architecture and storage ${ }^{49}$. Recently the Dutch Cancer Society Koningin Wilhelmina Fonds (KWF) awarded a grant of $€ 1.5$ million to Maastro clinic, a radiotherapy depertment at Maastricht and University Medical Centre Groningen (UMCG). They will set up a research infrastructure for proton therapy (PROTRAIT), working closely together with Holland Particle Therapy Center (HPTC) and all the other university medical centres.

We are now in a unique situation of expansion of the number of proton facilities throughout Europe and not enough radiation oncologists with long-term proton experience to work in them. Since most of the current experienced radiation oncologist received only limited education on particle therapy, there is an immense need for collaboration and sharing of knowledge with the experienced centres. Standardizing the follow up within the EPTN with regard to imaging, blood samples and research as well as daily patient care could be an important first step. Working together on a large scale by organizing educational sessions, internships, sharing our data safely open access and reporting our outcome on a large scale will become even more important than ever, to make particle therapy a part of the radiation oncologist wide pallet of treatment options.

With increasing survival rates after cancer treatment due to the combined efforts of doctors and researchers all over the world, an increasing number of survivors are subject to experience radiation induced long-term side effects ${ }^{50}$. Radiation induced secondary cancer is one of them, of special interest in those patients with a pathologically benign disease, such as meningioma or epilepsy, or with pediatric tumors 
treated with radiotherapy. With the increasing use of IMRT and VMAT techniques a low dose bath is administered to a large volume, not exceeding any tolerance dose $\mathrm{e}^{51,52}$. Combined with patient related factors, e.g., age, gender, treatment of primary cancer (systemic treatment) and genetic disorders, second cancers associated with radiation are most often seen in proximity of the irradiated area and after a long latency period, on average between 10 and 20 years ${ }^{52}$. The integral dose from particle therapy is beneficially on average 2-3 times lower than from photon therapy ${ }^{54}$. On the contrary nuclear interaction of protons create neutrons mainly within the patient, assuming pencil beam scanning as is the standard in The Netherlands, which induces a low dose everywhere in the patient. When passive scatter technique is used even more neutrons are produced. Which parameters will prove to correlate the best with secondary tumour induction is yet undetermined and needs to be subject to further research.

Another long term side effect of interest is neurocognitive decline with a long latency period up to 12 years ${ }^{55,56}$. In the search for OARs related to cognition we suggested the posterior cerebellum as a possible new OAR in this thesis ${ }^{25}$. Since the cerebellum receives unintentional low dose in patients treated for HNSCC, this is the ideal patient group to prospectively evaluate neurocognitive functioning before and after radiotherapy with standardized cognitive testing. More cognition related structures besides hippocampi or posterior cerebellum need to be determined. With the proposal of our atlas for neuro-oncology, the workload for the technician and radiation oncologist further increases. Future auto segmentation based on this consensus atlas will help to speed this process. This should be strived for my academic centres and companies specialised in image analysis. Future new structures possibly related to cognition need to be identified and integrated in the atlas, ensuring uniform delineation and dose registration. It will remain difficult to determine which OAR is the most dominant factor and needs to be spared as much as possible, especially if those OARs are located adjacent to each other receiving comparable dose during treatment. Radiation is not the only factor influencing cognition, systemic therapy, surgery, medication and also patient factors, e.g., stress, anxiety and fatigue can cause cognitive decline. Another problem with the development of a NTCP model for cognition is the standardization of cognitive testing. There are several domains to be tested and in an individual patient the most relevant tests would be selected depending on patient related factors, e.g., tumour location, specific post-operative deficits and age. In order to collect data for future NTCP models, cognitive testing and imaging should also be performed in a standardized way. Within the EPTN, subgroup working on standardisation of neuro-oncology is now preparing a consensus paper on the follow up 
of all to the aforementioned selected OARs and their related toxicity after radiotherapy including cognitive testing and imaging.

In this era of increasing number of patients treated with proton radiotherapy and growing follow up time, there is some concern about potential proton specific side effects, which clearly need further attention. Using particles the potential to induce a biological effect in cells, also called the relative biological effectiveness (RBE) is assumed to be larger; the RBE for carbon ions, protons and photons being of 3.0, 1.1 and 1.0 respectively. In the review of Lühr et $a I^{.57}$ studies are presented suggesting that the RBE of protons might be higher than 1.1, possibly varying on the position relative to the Bragg-peak, characterized by an increased linear energy transfer (LET). In daily practice this RBE of 1.1 is used for proton radiotherapy, according to the recommendation of the ICRU 78. Carefully illustrated by Lühr et al. ${ }^{57}$ pre-clinical studies consider the RBE a complex function of radiation dose, LET, cell type, endpoint etc. showing a cell survival from 1.1 at the spread out Bragg-peak (SOBP) entrance to even 4-6 in the distal fall off at $2 \mathrm{~Gy}$ per fraction ${ }^{57,58}$. They rightfully suggest 4 approaches to enhance proton therapy and deliver safe treatments. Step 1 being the awareness of RBE uncertainties during plan evaluation, step 2 mitigate the RBE effect by modification of beam positioning, step 3 generate clinical data by analysing patient outcome data and step 4 initiation of clinical relevant in vivo RBE studies ${ }^{57}$. On top of that, we suggest a fifth step: incorporating these data into proton treatment planning systems. This process should be accelerated in close collaboration with the clinicians and radiobiologists involved in the EPTN network as well as vendors of proton treatment planning systems.

The relevance of registration and reduction of side effects is also illustrated by the toxicity observation in our epilepsy review showing permanent toxicity, such as radionecrosis, cognitive and (asymptomatic) visual field deficit. Since there are no conclusive data on the $\alpha / \beta$, quantifying for fractionation sensitivity of tissues, e.g. epileptogenic lesions, future research should include fractionated radiotherapy schedules in order to investigate whether this leads to a reduction in toxicity compared to a single fraction, maintaining the reduction of seizure frequency. In an attempt to further reduce the toxicity of this pathologically benign disease, a randomized or at least a prospective registry study is indicated investigating the role of proton versus photon radiotherapy.

In conclusion there is still work to be done, to gain in depth knowledge of the full potential of particle radiotherapy, whether this concerns possible reduction of side 
effects, increased tumour control or variable RBE. Through further elaboration of current collaborations throughout the radiotherapy community together with all our colleagues within the medical field, there is a unique possibility to alter the side effects of our patients like a proton: positive! 


\section{References}

1. Beetz I, Schilstra C, van der Schaaf A, van den Heuvel ER, Doornaert P, van Luijk P, Vissink A. et al. NTCP models for patient-rated xerostomia and sticky saliva after treatment with intensity modulated radiotherapy for head and neck cancer: the role of dosimetric and clinical factors. Radiother Oncol 2012;105(1):101-6.

2. Dijkema T, Terhaard CH, Roesink JM, Braam PM, van Gils CH, Moerland MA, Raaijmakers CP. Large cohort dose-volume response analysis of parotid gland function after radiotherapy: intensitymodulated versus conventional radiotherapy. Int J Radiat Oncol Biol Phys 2008;72(4):1101-9.

3. Dijkema T, Raaijmakers CP, Ten Haken RK, Roesink JM, Braam PM, Houweling AC, et al. Parotid gland function after radiotherapy: the combined Michigan and Utrecht experience. Int J Radiat Oncol Biol Phys 2010;78(2):449-53.

4. Christianen ME, Schilstra C, Beetz I, Muijs CT, Chouvalova O, Burlage FR, et al. Predictive modelling for swallowing dysfunction after primary (chemo)radiation: results of a prospective observational study. Radiother Oncol 2012;105(1):107-14.

5. Langendijk JA, Lambin P, De Ruysscher D, Widder J, Bos M, Verheij M. Selection of patients for radiotherapy with protons aiming at reduction of side effects: the model-based approach. Radiother Oncol 2013;107(3):267-73.

6. Widder J, van der Schaaf A, Lambin P, Marijnen CA, Pignol JP, Rasch CR, Slotman BJ, et al. The Quest for Evidence for Proton Therapy: Model-Based Approach and Precision Medicine. Int J Radiat Oncol Biol Phys 2016;95(1):30-6.

7. Ramaekers BL, Grutters JP, Pijls-Johannesma M, Lambin P, Joore MA, Langendijk JA. Protons in headand-neck cancer: bridging the gap of evidence. Int J Radiat Oncol Biol Phys 2013;85:1282-8.

8. Lambin P, Petit SF, Aerts HJ, van Elmpt WJ, The ESTRO, Lecture Breur, et al. From population to voxelbased radiotherapy: exploiting intra-tumour and intra-organ heterogeneity for advanced treatment of non-small cell lung cancer. Radiother Oncol 2009;2010:145-52.

9. Lambin $\mathrm{P}$, van Stiphout RG, Starmans $\mathrm{MH}$, et al. Predicting outcomes in radiation oncologymultifactorial decision support systems. Nat Rev Clin Oncol 2013;10:27-40.

10. Lambin P, Roelofs E, Reymen B, et al. Rapid Learning health care in oncology' - an approach towards decision support systems enabling customized radiotherapy'. Radiother Oncol 2013;109:159-64.

11. Lambin $\mathrm{P}$, Zindler J, Vanneste $\mathrm{B}$, et al. Modern clinical research: how rapid learning health care and cohort multiple randomised clinical trials complement traditional evidence based medicine. Acta Oncol 2015;54:1289-300.

12. Eekers DBP, Roelofs E, Jelen U, Kirk M, Granzier M, Ammazzalorso F, et al. Benefit of particle therapy in re-irradiation of head and neck patients. Results of a multicentric in silico ROCOCO trial. Radiother Oncol 2016;121(3):387-94.

13. Eekers DBP, Roelofs E, Cubillos-Mesias M, Niel, C, Smeenk RJ, Hoeben A, et al. Intensity-modulated proton therapy decreases dose to organs at risk in low-grade glioma patients: results of a multicentric in silico ROCOCO trial. In press Acta Oncol 2018.

14. Grün R, Friedrich $T$, Krämer $M$, et al. Assessment of potential advantages of relevant ions for particle therapy: a model based study. Med Phys 2015;42:1037-47.

15. Takagi M, Demizu Y, Hashimoto N, et al. Treatment outcomes of particle radiotherapy using protons or carbon ions as a single-modality therapy for adenoid cystic carcinoma of the head and neck. Radiother Oncol 2014;113:364-70.

16. Koca T, Basaran H, Sezen D, Karaca S, Ors Y, Arslan D, Aydin A. Comparison of linear accelerator and helical tomotherapy plans for glioblastoma multiforme patients. Asian Pac J Cancer Prev 2014;15(18):7811-6.

17. Hermans BC, Persoon LC, Podesta M, Hoebers FJ, Verhaegen F, Troost EG. Weekly kilovoltage conebeam computed tomography for detection of dose discrepancies during (chemo)radiotherapy for head and neck cancer. Acta Oncol 2015;54:1483-9.

18. Steiner E, Stock M, Kostresevic B, et al. Imaging dose assessment for IGRT in particle beam therapy. Radiother Oncol 2013;109:409-13. 
19. Kraan AC, Van de Water S, Teguh DN. Dose uncertainties in IMPT for oropharyngeal cancer in the presence of anatomical, range, and setup errors. Int J Radiat Oncol Biol Phys 2013;87:888-96.

20. Müller BS, Duma MN, Kampfer S, et al. Impact of interfractional changes in head and neck cancer patients on the delivered dose in intensity modulated radiotherapy with protons and photons. Phys Med 2015;31:266-72.

21. Jakobi A, Bandurska-Luque A, Stützer K, et al. Identification of patient benefit from proton therapy for advanced head and neck cancer patients based on individual and subgroup normal tissue complication probability analysis. Int J Radiat Oncol Biol Phys 2015;92:1165-74.

22. Richard P, Sandison G, Dang Q, Johnson B, Wong T, Parvathaneni U. Dental amalgam artefact: adverse impact on tumor visualization and proton beam treatment planning in oral and oropharyngeal cancers. Pract Radiat Oncol 2015;5:e8-e583.

23. Giantsoudi D1, De Man B, Verburg J, Trofimov A, Jin Y, Wang G, et al. Metal artifacts in computed tomography for radiation therapy planning: dosimetric effects and impact of metal artifact reduction. Phys Med Biol 2017;62(8):R49-R80.

24. Harrabi SB, Bougatf N, Mohr A, Haberer T, Herfarth K, Combs SE, et al. Dosimetric advantages of proton therapy over conventional radiotherapy with photons in young patients and adults with low-grade glioma. Strahlenther Onkol 2016;192(11):759-769.

25. Eekers DBP, In 't Ven L, Deprez S, Jacobi L, Roelofs E, Hoeben A, et al. The posterior cerebellum, a new organ at risk? Clin Transl Radiat Oncol. 2017;8:22-26.

26. Ribi K, Relly C, Landolt MA, Alber FD, Boltshauser E, Grotzer MA. Outcome of medulloblastoma in children: long-term complications and quality of life. Neuropediatrics 2005;36(6):357-65.

27. Gondi V, Hermann BP, Mehta MP, Tomé WA. Hippocampal dosimetry predicts neurocognitive function impairment after fractionated stereotactic radiotherapy for benign or low-grade adult brain tumors. Int J Radiat Oncol Biol Phys 2013;85(2):348-54.

28. Rapoport M, van Reekum R, Mayberg $H$. The role of the cerebellum in cognition and behavior: $a$ selective review. J Neuropsychiatry Clin Neurosci 2000;12 (2):193-8.

29. Schmahmann JD, Doyon J, McDonald D, Holmes C, Lavoie K, Hurwitz AS, et al. Three-dimensional MRI atlas of the human cerebellum in proportional stereotaxic space. Neuroimage 1999;10(3):233-60.

30. Merchant TE, Sharma S, Xiong X, Wu S, Conklin H. Effect of cerebellum radiation dosimetry on cognitive outcomes in children with infratentorial ependymoma. Int J Radiat Oncol Biol Phys 2014;90(3):547-53.

31. Gan HK, Bernstein L, Brown J, Ringash J, Vakilha M, Wang L, et al. Cognitive functioning after radiotherapy or chemoradiotherapy for head-and-neck cancer. Int J Radiat Oncol Biol Phys 2011;81(1): 126-34.

32. Eekers DBP, in 't Ven L, Roelofs E, Postma A, Troost EGC. EPTN International Neurological Contouring Atlas. CancerData 2017. doi:10.17195/candat.2017.08.1.

33. Eekers DBP, in 't Ven L, Roelofs E, Postma A, Alapetite C, Burnet N, et al. The EPTN consensus-based atlas for CT- and MR-based contouring in Neuro-Oncology. "European Particle Therapy Network" of ESTRO. Radiother Oncol 2018; pii: S0167-8140(17)32768-8.

34. Rasch CR, Steenbakkers RJ, Fitton I, Duppen JC, Nowak PJ, Pameijer FA, et al. Decreased 3D observer variation with matched CT-MRI, for target delineation in Nasopharynx cancer. Radiat Oncol 2010;5:21.

35. Brouwer CL, Steenbakkers RJ, Bourhis J, Budach W, Grau C, Grégoire V, et al. CT-based delineation of organs at risk in the head and neck region: DAHANCA, EORTC, GORTEC, HKNPCSG, NCIC CTG, NCRI, NRG Oncology and TROG consensus guidelines. Radiother Oncol 2015;117(1):83-90.

36. Kong FM, Ritter T, Quint DJ, Senan S, Gaspar LE, Komaki RU, et al. Consideration of dose limits for organs at risk of thoracic radiotherapy: atlas for lung, proximal bronchial tree, esophagus, spinal cord, ribs, and brachial plexus. Int J Radiat Oncol Biol Phys 2011;81:1442-57.

37. Vinod SK, Jameson MG, Min M, Holloway LC. Uncertainties in volume delineation in radiation oncology: A systematic review and recommendations for future studies. Radiother Oncol. 2016;121(2):169-79.

38. Bartel F, van Herk M, Vrenken $\mathrm{H}$, Vandaele F, Sunaert S, de Jaeger $\mathrm{K}$, et al. Inter-observer variation of hippocampus delineation in hippocampal avoidance prophylactic cranial irradiation. Clin Transl Oncol 2018 Jun 6. 
39. Gondi V, Tome WA, Rowley HA, Mehta MP. Hippocampal Contouring: A Contouring Atlas for RTOG 0933 n.d. https ://www.rtog.org/CoreLa b/Contou ringA tlase s/Hippoc ampa ISpar ing.aspx. Accessed 25 Sept 2017).

40. Santanam L, Hurkmans C, Mutic S, van Vliet-Vroegindeweij C, Brame S, Straube W, et al. Standardizing naming conventions in radiation oncology. Int J Radiat Oncol Biol Phys 2012;83:1344-9.

41. Lambrecht M, Eekers DBP, Alapetite C, Burnet NG, Calugaru V, et al. Radiation dose constraints for organs at risk in neuro-oncology; the European Particle Therapy Network consensus. Radiother Oncol. 2018 2018;128(1):26-36.

42. Eekers DBP, Lambrecht M, Nyström PDW, Swinnen A, Wesseling FWR, Roelofs E, Troost EG. EPTN consensus-based guideline for the tolerance dose per fraction of organs at risk in the brain. CancerData, 2018; doi:10.17195/candat.2018.01.1.

43. Perkó Z, Bortfeld TR, Hong TS, Wolfgang J, Unkelbach J. Derivation of mean dose tolerances for new fractionation schemes and treatment modalities. Phys Med Biol. 2018 Feb 5;63(3):035038.

44. Hoffmann AL, Nahum AE. Fractionation in normal tissues: the $(\alpha / \beta)$ eff concept can account for dose heterogeneity and volume effects. Phys Med Biol. 2013 Oct 7;58(19):6897-914.

45. Eekers DBP, Pijnappel EN, Schijns OEMG, Colon A, Hoeben A, Zindler, et al. Evidence on the efficacy of primary radiosurgery or stereotactic radiotherapy for drug-resistant non-neoplastic focal epilepsy in adults: a systematic review. Seizure. 2018;55:83-92.

46. Cook MJ. "Truths and roses have thorns about them".Epilepsia. 2018 Jun;59(6):1208-1209.

47. Barbaro NM, Quigg M, Ward MM, Chang EF, Broshek DK, Langfitt JT. et al. Radiosurgery versus open surgery for mesial temporal lobe epilepsy: The randomized, controlled ROSE trial. Epilepsia. 2018 Jun;59(6):1198-1207.

48. Ogino T. Clinical evidence of particle beam therapy (proton). Int J Clin Oncol. 2012 Apr;17(2):79-84.

49. Skripcak T, Belka C, Bosch W, Brink C, Brunner T, Budach V, et al. Creating a data exchange strategy for radiotherapy research: towards federated databases and anonymised public datasets. Radiother Oncol. 2014 Dec;113(3):303-9.

50. Schneider U. Modeling the risk of secondary malignancies after radiotherapy. Genes (Basel). 2011 Nov 29;2(4):1033-49.

51. Followill D, Geis P, Boyer A. Estimates of whole-body dose equivalent produced by beam intensity modulated conformal therapy. Int. J. Radiat. Oncol. Biol. Phys. 1997, 38, 667-672; Erratum. Int. J. Radiat. Oncol. Biol. Phys. 1997, 39, 783.

52. Hall EJ, Wuu CS. Radiation-induced second cancers: The impact of 3D-CRT and IMRT. Int. J. Radiat. Oncol. Biol. Phys. 2003, 56, 83-88.

53. Bhatia, S.; Sklar, C. Second cancers in survivors of childhood cancer. Nat. Rev. Cancer 2002, 2, 124-132.

54. Lomax AJ, Bortfeld T, Goitein G, Debus J, Dykstra C, Tercier PA, et al. A treatment planning intercomparison of proton and intensity modulated photon radiotherapy. Radiother. Oncol. 1999, 51, 257271.

55. Klein M, Heimans JJ, Aaronson NK, van der Ploeg HM, Grit J, Muller M, et al. Effect of radiotherapy and other treatment-related factors on mid-term to long-term cognitive sequelae in low-grade gliomas: a comparative study. Lancet. 2002 Nov 2;360(9343):1361-1368.

56. Douw L, Klein M, Fagel SS, van den Heuvel J, Taphoorn MJ, Aaronson NK, et al. Cognitive and radiological effects of radiotherapy in patients with low-grade glioma: long-term follow-up. Lancet Neurol. 2009 Sep;8(9):810-818.

57. Lühr A, von Neubeck C, Krause M, Troost EGC. Relative biological effectiveness in proton beam therapy Current knowledge and future challenges. Clin Transl Radiat Oncol. 2018 Feb 1;9:35-41.

58. Paganetti H. Relative biological effectiveness (RBE) values for proton beam therapy. Variations as a function of biological endpoint, dose, and linear energy transfer. Phys Med Biol 2014;59:R419-472. 
Valorisation 



\section{Valorisation}

Since the introduction of radiotherapy for the treatment of cancer, more than hundred years ago, doctors have been trying to report treatments results as systematically and precisely as possible in order to learn about tumour as well as healthy tissue response to radiotherapy. The ultimate goal of radiotherapy is to eradicate the tumour without any lasting toxicity. In order to achieve this ambitious goal, several approaches are possible.

One approach being subject of this thesis is reduction of the actual dose to the healthy tissue surrounding the tumour also known as the organs at risk (OARs). This reduction of dose can be achieved by using state of the art irradiation techniques such as intensity-modulated radiotherapy (IMRT) in which the intensity of the dose within the beam can be modulated resulting in an adequate dose to the tumour and an as low as possible dose to the OARs. Thanks to evolving technical possibilities, there are several ways to use IMRT in benefit of the patient, for example volumetric modulated arc technique (VMAT) and helical tomotherapy (TOMO). Another way to reduce dose to the OARs is using particle therapy instead of photon therapy, in which charged particles, for example protons and carbon ions, are used for irradiation. Due to specific characteristics of these particles the dose to surrounding healthy tissue can be reduced in some patients. Whether this reduction in dose to the OARs is clinically relevant has to be proven in time. Since proton therapy is becoming more and more available, although still on a small scale, the need for optimal patient selection becomes warranted since only a limited number of patients can be treated in such facilities.

There are also extra costs involved with particle therapy compared to photon radiotherapy. This is why in silico studies are the logical first step to investigate whether a dosimetric advantage is achievable and effective. In Chapter $\mathbf{2}$ and $\mathbf{3}$ head and neck and low-grade glioma patients are included the two separate in silico trials conducted within the Radiation Oncology Collaborative Comparison (ROCOCO) consortium. These studies demonstrated the benefit of particle therapy through simulation instead of actual treatment.

Thanks to these in silico trials, we know there is a dosimetric advantage for particle therapy, but it remains unsure whether this translates into a clinical benefit. To this end, an accurate prediction of the effect of radiation on various tissues is essential, such as the prediction of tumour response related to the delivered dose, also known as the tumour control probability (TCP). For many tumours, a higher dose is related to a better local control. This of course needs to be weighed against the inevitable exposure of the surrounding healthy tissue to radiation causing side effects, known as the normal 
tissue complication probability (NTCP). Both TCP and NTCP are dependent on various patient-related factors of which some are not yet identified, especially in case of reirradiation, but also for central nervous system (CNS) side effects, specifically. When TCP and NTCP are known for each type of tissue (tumour and healthy tissue) a wellbalanced treatment choice can be made based on the risk of side effects versus the possibility of achieving local control of the tumour per treatment modality.

Many different radiation centres started collecting dosimetric and toxicity data decades ago. Surprisingly, despite these great efforts in the past, there is still a desperate need for validated TCP and NTCP models, especially in the field of neuro-oncology. One of the reasons the collected data did not result in proper validation of such prediction models is because they lack uniformity of the actual collected data. It is therefore of great importance that the relevant CNS anatomical structures are identified and uniformly delineated in order to uniformly report the received dose related to registered side effects. For this a consensus-based delineation atlas, which includes all (potentially) relevant OARs, is needed. This thesis provides in this need by producing a European Particle Therapy Network (EPTN) consensus-based Neuro-Oncology Atlas including the posterior cerebellum (Chapter $\mathbf{4}$ and $\mathbf{5}$ ) as a new potentially relevant OAR. This neuro-oncology atlas will enable photon and particle radiotherapy centres within Europe and beyond to uniformly report on dose related toxicity for each specific OAR. Since manual delineation of OARs is one of the most time consuming task in radiotherapy planning and subject to inter- and intra-observer variability, there is a great interest in developing an automated delineation atlas. The CNS atlas presented in this thesis will serve as a base for this, e.g. for the Danish head and neck group (DAHANCA) and Danish brain oncology group (DNOG).

When there is agreement on the relevant OARs and the structure names and delineation thereof, the next important issue is agreement on the dose each OAR tolerates without long-term toxicity, based on available literature. This agreement is important since the literature on tolerance dose is scarce, causing room for individual interpretation. This results in treatment plans being based on different, by radiation oncologists accepted doses in the OAR obstructing accurate treatment modality comparison. The latter is needed to upfront select the best treatment option for each individual patient. This thesis provides in this need by reaching this agreement by producing an EPTN consensus CNS Tolerance Table (Chapter 6). Both the CNS Delineation Atlas as well as the Tolerance Dose table are now incorporated into daily practice guidelines, as well as in trial protocols within and outside of the EPTN.

This year, the first patient was treated with proton therapy in The Netherlands and health insurance companies together with the government and experts in the field of 
radiotherapy will continue to work together to improve patient selection by collecting actual toxicity data in a mutual database. Collecting these data in a uniform matter will be the next challenging step. Especially for neuro oncology, were cognitive decline after radiotherapy is of great importance, uniform cognitive testing is needed, for example. As a result of chapter 4, 5 and 6, an EPTN expert group is now continuing previous work by producing a consensus on this toxicity registration. This will eventually result in the possibility to uniformly combine CNS dosimetric and toxicity data from all different centres throughout the world, enabling efficient cooperation between experts to produce and validate the needed NTCP models within the coming years.

Another good example of cooperation leading to optimal treatment results is the treatment of epilepsy. Epilepsy in general is one of the most common severe neurological disorders. It is a disease in which patients have unexpected seizures making participation in daily family life and working processes challenging, which can have severe economic consequences. If the epilepsy is resistant to anti-epileptic drugs, resection of the epileptogenic region is an invasive treatment option for only a small group of patients. Currently, there is a need for non-invasive alternative treatments, especially for those patients not eligible for radical surgery. Chapter 7 shows that radiotherapy is such an alternative treatment for epilepsy. Due to the excellent collaboration between the neurologist, neurosurgeon, neuro-radiologist and radiotherapist within the southern part of The Netherlands, and having the best radiotherapy techniques, the next logical step will be offering radiotherapy to a selected group of epilepsy patient in Maastricht for the first time in the Netherlands. 

Summary 



\section{Summary}

The aim of this thesis is to further optimise radiation therapy of Brain and Head \& Neck by reducing the dose to the healthy surrounding tissue, so called organs at risk (OARs), leading to a reduction in side effects

The first objective of this doctoral thesis was to assess the value of proton therapy in reducing the dose to the OARs, in particular for re-irradiation in head and neck squamous cell carcinoma and primary irradiation of low-grade glioma. Chapters $\mathbf{2}$ and $\mathbf{3}$ report on two in silico trials conducted within the international Radiation Oncology Collaborative Comparison (ROCOCO) consortium, comparing different radiotherapy modalities including proton therapy, to assess the potential gains for individual patients due to the dosimetric characteristics of particle therapy. The first trial compared intensity-modulated proton therapy (IMPT) and carbon-ion therapy (IMIT) with the golden standard volumetric modulated arc therapy (VMAT) when re-irradiating patients with head and neck squamous cell carcinoma. The second trial compared intensitymodulated radiation therapy (IMRT), IMPT and helical tomotherapy (TOMO) with the golden standard VMAT in patients with a low-grade glioma. Both trials demonstrated that particle therapy can significantly reduce the dose to OARs whilst maintaining the prescription dose. Whether this translates into a clinically relevant benefit, is the subject of future research. In order to predict such a benefit, normal tissue complication probability (NTCP) models are needed. Validated NTCP models are currently lacking for head and neck re-irradiation as well as for the primary treatment of the central nervous system (CNS).

In Chapter 4, the posterior cerebellum is introduced as a new, potentially relevant OAR for the future development of an NTCP model that is focused on cognition, based on the growing evidence from structural and functional imaging studies that the cerebellum plays a role in neurocognition.

Delineation of the relevant OARs on computed tomography (CT) and magnetic resonance imaging (MRI) is needed to optimise the treatment plan before administering the corresponding dose to the patient. This manual delineation is a timeconsuming process and a well-known source of error within the planning process, due to inter- and intra-observer variability ${ }^{1}$.

Reducing this CNS OAR delineation variability is the second objective of this thesis, as described in Chapter 5. An international group of expert radiation oncologists in the field of neuro-oncology reached agreement on the European Particle Therapy Network 
(EPTN) consensus-based CNS delineation atlas, in order to decrease the variation in CNS delineation. This CNS atlas is presented online (www.cancerdata.org) and encompasses delineation instructions for 15 CNS OARs, including the posterior cerebellum. It includes one CT-scan at two different brightness and contrast settings and two MR scans ( 3 and 7 Tesla) showing the OARs in three directions (axial, coronal and sagittal view). In Chapter 6, an EPTN consensus-based normal tissue tolerance table, including all currently known and deemed relevant CNS OARs, reports the tolerance dose in equivalent dose (EQD2), which enables a uniform comparison of different treatment modalities in the future (www.cancerdata.org). The use of the consensus-based EPTN CNS atlas and tolerance table is recommended for the Dutch model-based approach comparing photon and proton beam irradiation as well as for future prospective clinical trials including novel radiation techniques and/or modalities ${ }^{2}$.

The third objective of this thesis was determining the role of radiotherapy in the treatment of epilepsy. Chapter 7 contains a systematic review about the evidence on the efficacy of primary radiosurgery or stereotactic radiotherapy for drug-resistant nonneoplastic focal epilepsy in adults. After treatment, an average of $58 \%$ of the patients reported no or rare seizures (defined as radiotherapy-adapted Engel class [RAEC] I and II). A dose-effect model was fitted to the available response data to derive a relationship between prescribed dose and RAEC frequency of this, in the Netherlands, new indication for radiotherapy. In Chapter $\mathbf{8}$, the results of the previous mentioned chapters of this thesis are being discussed and future perspectives are presented. Additional research needs to be conducted to gain further knowledge to fully understand the potential of particle therapy. Thanks to solid collaborations throughout the radiotherapy community and beyond, with all our colleagues in the medical field, there is a unique possibility to further optimise the treatment of Brain and Head \& Neck together. 


\section{References}

1. Vinod SK, Jameson MG, Min M, Holloway LC. Uncertainties in volume delineation in radiation oncology: A systematic review and recommendations for future studies. Radiother. Oncol 2016;121:169-79.

2. Widder J, Schaaf van der A, Lambin P, et al. The quest for evidence for proton therapy: the model-based approach and precision medicine. Int J Radiat Oncol Biol Phys 2015. pii: S0360-3016:26569-8. 


\section{Samenvatting}

Het doel van dit proefschrift is de verdere optimalisatie van de radiotherapeutische behandeling van aandoeningen in hersenen en hoofd $\&$ hals door de dosis te verlagen in het gezonde omringende weefsel, de zogenaamde risico-organen (OAR's), wat leidt tot een vermindering van bijwerkingen.

Het eerste doel van dit proefschrift was het beoordelen van de waarde van protontherapie bij het verlagen van de dosis in de OAR's, in het bijzonder voor hernieuwde bestraling bij plaveiselcelcarcinoom van hoofd \& hals en de primaire bestraling van laaggradige gliomen in de hersenen. Hoofdstukken $\mathbf{2}$ en $\mathbf{3}$ rapporteren over twee in silico-onderzoeken die zijn uitgevoerd binnen het internationale Radiation Oncology Collaborative Comparison (ROCOCO) consortium, waarbij verschillende radiotherapie modaliteiten, waaronder protonentherapie, worden vergeleken om de potentiële voordelen voor individuele patiënten te bepalen vanwege de dosimetrische kenmerken van deeltjestherapie. Het eerste onderzoek vergeleek intensiteit gemoduleerde protonentherapie (IMPT) en koolstof-ionentherapie (IMIT) met de gouden standaard, zijnde volumetrisch gemoduleerde boogtherapie (VMAT) bij het opnieuw bestralen van patiënten met plaveiselcelcarcinoom van hoofd \& hals. In het tweede onderzoek werden intensiteit gemoduleerde bestralingstherapie (IMRT), IMPT en helical tomotherapy (TOMO) vergeleken met de gouden standaard VMAT bij patiënten met een laaggradig glioom. Beide onderzoeken toonden aan dat deeltjes therapie de dosis aanzienlijk kan verlagen in de OAR's terwijl de voorgeschreven dosis op de tumor gehandhaafd blijft. Of dit zich vertaalt in een klinisch relevant voordeel, is onderwerp van verder onderzoek. Om een dergelijk voordeel te voorspellen, zijn modellen nodig die de kans op complicaties aan gezond weefsel voorspellen (NTCPmodellen). Gevalideerde NTCP-modellen ontbreken momenteel voor hernieuwde bestraling van hoofd \& hals en voor de primaire behandeling van het centrale zenuwstelsel (CZS).

In Hoofdstuk 4 wordt het achterste deel van de kleine hersenen (posterior cerebellum) geïntroduceerd als een nieuw, potentieel relevant OAR voor de toekomstige ontwikkeling van een NTCP-model dat gericht is op cognitie. Er is groeiend bewijs op basis van structurele en functionele beeldvormingsstudies dat het posterieure cerebellum een rol speelt bij neurocognitie. 
De delineatie van de relevante OAR's op computertomografie (CT) en magnetische resonantie beeldvorming (MRI) is nodig om het behandelplan te optimaliseren voordat de overeenkomstige dosis aan de patiënt wordt toegediend. Deze handmatige delineatie is een tijdrovend proces en een bekende bron van fouten binnen het planningsproces, vanwege inter- en intra-waarnemer variabiliteit ${ }^{1}$.

Het reduceren van deze CNS OAR-delineatie variabiliteit is de tweede doelstelling van dit proefschrift, zoals beschreven in Hoofdstuk 5. Een internationale groep van deskundige Radiotherapeut Oncologen op het gebied van neuro-oncologie bereikten overeenstemming over de European Particle Therapy Network (EPTN) consensusgebaseerde CNS-delineatie atlas, om de variatie in CNS-delineatie te verminderen. Deze CNS-atlas wordt online gepresenteerd (www.cancerdata.org) en omvat de delineatieinstructies voor 15 CNS OAR's, inclusief het posterior cerebellum. Het bevat één CTscan met twee verschillende helderheid en contrast instellingen en twee MR-scans met verschillende veldsterkten ( 3 en 7 Tesla) die de OAR's in drie richtingen weergeven (axiaal, coronaal en sagittaal). Hoofdstuk 6 rapporteert een EPTN-consensus tolerantie tabel voor normale weefsels met inbegrip van alle momenteel bekende en relevant geachte CNS OAR's en hun tolerantiedosis in equivalente dosis (EQD2) waardoor een uniforme vergelijking van verschillende behandelmodaliteiten in de toekomst mogelijk is geworden (www.cancerdata.org). Het gebruik van de consensus gebaseerde EPTN CNS-atlas en tolerantietabel wordt aanbevolen voor de Nederlandse model gebaseerde benadering ("model-based approach") waarbij fotonen- en protonen bestraling wordt vergeleken, evenals voor toekomstige prospectieve klinische onderzoeken met inbegrip van nieuwe stralingstechnieken en / of -modaliteiten ${ }^{2}$.

De derde doelstelling van dit proefschrift was het bepalen van de rol van radiotherapie bij de behandeling van epilepsie. Hoofdstuk 7 bevat een systematische review van het bewijsmateriaal over de werkzaamheid van primaire radiochirurgie of stereotactische radiotherapie voor niet-neoplastische focale epilepsie bij volwassenen die resistente zijn tegen geneesmiddelen. Na behandeling rapporteerde gemiddeld $58 \%$ van deze patiënten geen of zeldzame epilepsieaanvallen (gedefinieerd als radiotherapieaangepaste Engel-klasse [RAEC] I en II). Een dosis-effectmodel werd gemaakt gebaseerd op deze uit de literatuur beschikbare gegevens waarbij een verband gelegd wordt tussen de voorgeschreven dosis en de epilepsie (RAEC) frequentie, voor deze in Nederland nieuwe radiotherapie indicatie. In hoofdstuk 8 worden de resultaten van de eerdergenoemde hoofdstukken van dit proefschrift besproken en worden toekomstperspectieven gepresenteerd. Bijkomend onderzoek moet worden uitgevoerd om meer kennis te verkrijgen om het potentieel van deeltjestherapie volledig te 
begrijpen. Dankzij solide samenwerkingsverbanden in de gehele radiotherapie gemeenschap en daarbuiten, met al onze collega's van andere vakgebieden, is er een unieke mogelijkheid om de behandeling van hersenen en hoofd \& hals samen verder te optimaliseren. 


\section{Referenties}

1. Vinod SK, Jameson MG, Min M, Holloway LC. Uncertainties in volume delineation in radiation oncology: A systematic review and recommendations for future studies. Radiother. Oncol 2016;121:169-79.

2. Widder J, Schaaf van der A, Lambin P, et al. The quest for evidence for proton therapy: the model-based approach and precision medicine. Int J Radiat Oncol Biol Phys 2015. pii: S0360-3016:26569-8. 

Dankwoord 



\section{Dankwoord}

Het succesvol afronden van een proefschrift doe je niet alleen. Hieronder de mensen waar mijn speciale dank naar uit gaat.

Prof. dr. Philippe Lambin (promotor), voor het zien van de passie in mij en de kans en vrijheid die je me hebt gegeven me op wetenschappelijk gebied verder te ontwikkelen.

Prof. dr. Dirk de Ruysscher (promotor), voor je rustige vertrouwen in mij en het zien en aanspreken van mijn talenten. Onze samenwerking op wetenschappelijk gebied stopt gelukkig niet met het afronden van mijn promotie.

Prof. dr. dr. Esther Troost (promotor), voor het overbrengen van de kunst van het schrijven, je perfectie, enthousiasme, eerlijkheid en vriendschap. Jij verstaat de kunst een ander te laten excelleren.

Dr. Frank Hoebers (co-promotor), voor je vakkennis op het gebied van de hoofd hals oncologie en je blijvende interesse en stimulering tijdens mijn promotie.

Prof. dr. ir. Frank Verhaegen (voorzitter leescommissie), voor je tomeloze energie, gedrevenheid, humor en enorme kennis van ons mooie vak welke je moeiteloos deelt met jong en oud.

Corona leden (dr Jacoline Bromberg, dr. Albert Colon, prof. dr. Klaus Herfarth, prof. dr. Martin Klein, prof. dr. Maarten Lambrecht, prof. dr. Yasin Temel, dr. ir. Gloria VilchesFreixas, prof. dr. ir. Frank Verhaegen, prof. dr. Joachim Wildberger), voor de prettige samenwerking nu en in de toekomst, met de optimale kruisbestuiving tussen onze vakgebieden.

Prof. dr. Klaus Herfarth (leescommissie), für Ihre Gastfreundschaft und Offenheit beim Teilen Ihrer jahrelangen Erfahrung in der Partikeltherapie in Heidelberg (HIT).

Dr. Ann Hoeben, voor je vriendschap, inzicht en gedrevenheid. Je passie is inspirerend voor iedereen om je heen.

Dr. Linda Jacobi (A.A. Postma), voor je positief kritische hulp en de humor waarmee dit gepaard ging, waarvoor je altijd weer tijd wist vrij te maken. 
Dr. Albert Colon, dr. ir. Aswin Hoffmann, drs. Esther Pijnappel \& dr. Olaf Schijns, voor jullie enthousiasme en professionele gedrevenheid om samen te zoeken naar alternatieve behandelingen om de epilepsiepatiënt verder te helpen. Mooi dat onze prettige samenwerking zo tijdloos is gebleken.

ROCOCO members (Andre $\mathrm{M}$, Ann $\mathrm{H}$, Charles N, Erik R, Esther T, Filippo A, Frank $\mathrm{H}$, Geert J, Hans K, Lieke V, Macarena C-M, Marlies G, Maura K, Peter A, Philipppe L, Raquel DF, Robert Jan S, Sean W, Timothy S, Tobias F, Urszula J, Yvonne K), for investing your energy, ideas and time in this impressing international project nourished by Erik Roelofs, resulting in at least 4 PhDs!

EPTN CNS members, for all your enthusiasm and professional willingness to work together as a solid team of experts in the field of neuro-oncology.

Neuro-oncologie research team (Ann H, Ans S, Inge C, Jan B, Jaap Z, Karin T, Kasper R, Linda A, Linda J, Maikel V, Marc V, Martijn B, Mieke G, Monique A, Olaf S, Onno T, Piet $\operatorname{vd} \mathrm{E}, \mathrm{Rob} J)$, voor het laten zien wat een team samen kan bereiken.

Maastro medewerkers, voor de fijne samenwerking. In het bijzonder, team 4 voor het onderlinge respect, de humor en natuurlijk de heerlijke taarten en Mariëlle \& Natascha voor de enthousiaste en professionele secretariële ondersteuning.

Inge, Lieke \& Maikel, voor jullie enthousiasme; het doet me plezier jullie PhD-traject te mogen begeleiden.

Marie-Cécile \& Anja, voor jullie vriendschap en de gezellige lunches in en rondom ons geliefde Vijlen.

Protongirls (Judith, Karolien \& Stephanie), voor de proton typische sfeer (positief), gezellige etentjes $\&$ bakjes koffie/thee.

Paranimfen (Debby en Andrea), voor jullie jarenlange vriendschap. Jullie liefde, humor en openheid geeft mij steeds weer positieve energie.

Schoonouders (Luiksje en Jan), voor het herkennen en stimuleren van mijn liefde voor tuinieren en het verrijken van ons gezin met onze lieve viervoeters Rocky en ? 
Zussen (Patricia en Pascal), voor jullie lieve bemoedigende woorden, interesse en relativeringsvermogen. Patricia ook bedankt voor je altijd weer nuttige inhoudelijke feedback als gewaardeerd collega en Pascal voor het delen van je artistieke talent door het maken van de prachtige kaft "Emma".

Ouders (Mariet en Albert), voor jullie liefde en onuitputtelijk steun naast de stimulering om op eigen benen te staan en een eigen onderbouwde mening te vormen en deze respectvol te uiten. Jullie zijn de beste fanclub die een dochter zich maar wensen kan.

Kinderen (Karsten en Imke), voor jullie liefdevolle knuffels; samen met papa geven jullie de glans aan mijn leven. Karsten bedankt voor je fantastische humor, je altijd weer verrassende kennis en het samen Rocky uitlaten. Imke bedankt voor je zonnige lach, je heerlijke kookkunsten, het samen zwemmen, shoppen, vakanties plannen en binge-watchen.

En als laatste en belangrijkste ondersteunende factor in het behalen van mijn PhD: Erik. We hebben de afgelopen jaren vaak ook met veel plezier als collega's aan de keukentafel gezeten om onze research ideeën bij elkaar te toetsen en vorm te geven. Je bent een uitzonderlijk lieve en zorgzame vader en partner. Wat bof ik toch dat ik samen met jou door het leven mag gaan. 

List of publications 



\section{List of publications}

1. Eekers DBP, Roelofs E, Cubillos-Mesías M, Niël C, Smeenk RJ, Hoeben A, Minken AWH, Granzier M, Janssens GO, Kaanders JHAM, Lambin P, Troost EGC. Low-grade glioma patients: Results of a multicentric in silico ROCOCO trial”. Acta Oncologica 2018 in press.

2. Eekers DBP, Roelofs E, Cubillos-Mesías C, Niël C, Smeenk RJ, Hoeben A et al. Data from: Intensity-modulated proton therapy decreases dose to organs at risk in lowgrade glioma patients: results of a multicentric in silico ROCOCO trial. CancerData. 2018. doi:10.17195/candat.2018.09.1.

3. Schyns LEJR, van der Heyden B, AlmeidalP, Vaniqui A, Eekers DBP, Verhaegen F. Murine versus human tissue compositions: implications of using human tissue compositions for dose calculations in mice. Accepted BJR 2018.

4. Klaver YLB, van der Weide HL, Coremans IEM, Mendez Romero AM, Wiggenraad RGJ. Eekers DBP, Selection of brain tumour patients for proton therapy: The Dutch approach. WFNOS. 2018 in press

5. Schimmel WCM, Gehring K, Eekers DBP, Hanssens PEJ, Sitskoorn MM. Cognitive Effects of Stereotactic Radiosurgery in Adult Patients with Brain Metastases: A Systematic Review. IJROBP. 2018 in press. doi.org/10.1016/j.adro.2018.06.003

6. Witlox WJA, Ramaekers BLT, Zindler JD, Eekers DBP, van Loon, JGM, Le Pechoux, Hendriks L, Dingemans A-M, De Ruysscher DKM. The prevention of brain metastases in non-small cell lung cancer by prophylactic brain irradiation: Time to revisit? Front Oncol. 2018 Jul 26;8:241. doi: 10.3389/fonc.2018.00241

7. Lambrecht M, Eekers DBP, Alapetite C, Burnet NG, Calugaru V, Coremans IEM, Fossati P, Høyer M, Langendijk JA, Romero AM, Paulsen F, Perpar A, Renard L, de Ruysscher D, Timmermann B, Vitek P, Weber DC, van der Weide HL, Whitfield GA, Wiggenraad R, Roelofs E, Nyström PW, Troost EGC; work package 1 of the taskforce "European Particle Therapy Network" of ESTRO. Radiation dose constraints of organs at risk in neuro-oncology; the European Particle Network consensus. Radiother Oncol. 2018 Jul;128(1):26-36. doi: 10.1016/j.radonc.2018.05.001.

8. Eekers DBP, Lambrecht M, Nyström PDW, Swinnen A, Wesseling FWR, Roelofs E, Troost, EGC. EPTN consensus-based guideline for the tolerance dose per fraction of organs at risk in the brain. CancerData; 2018. doi:10.17195/candat.2018.01.1

9. Eekers DBP, In 't Ven L, Roelofs E, Postma A, Alapetite C, Burnet NG, Calugaru V, Compter I, Coremans IEM, Høyer M, Lambrecht M, Nyström PW, Romero AM, Paulsen F, Perpar A, de Ruysscher D, Renard L, Timmermann B, Vitek P, Weber DC, van der Weide HL, Whitfield GA, Wiggenraad R, Troost EGC; "European Particle 
Therapy Network" of ESTRO. The EPTN consensus-based atlas for CT- and MRbased contouring in Neuro-Oncology. Radiother Oncol. 2018 Jul;128(1):37-43.

10. Eekers DBP, in 't Ven L, Roelofs E, Postma A, Troost EGC. EPTN International Neurological Contouring Atlas. CancerData 2017. doi:10.17195/candat.2017.08.1.

11. van der Heyden B, Podesta M, Eekers DBP, Schyns LEJR, Vaniqui A, Almeida IP, van Hoof SJ, Verhaegen F. Automatic multi-atlas based organ at risk contouring in mice. Britisch Journal of Radiology June 2018. Br J Radiol 2018;91:20180364.

12. Eekers DBP, Pijnappel EN, Schijns OEMG, Colon A, Hoeben A, Zindler JD, Postma $A A$, Hoffmann AL, Lambin P, Troost EGC. Evidence on the efficacy of primary radiation therapy for drug-resistant localized non-neoplastic epilepsy in adults: a systematic review. Seizure. 2018 Feb;55:83-92.

13. Verduin M, Compter I, Steijvers D, Postma A, Eekers DBP, Anten M, Ackermans L, ter Laan M, Leijenaar RTH, van de Weijer T, Tjan-Heijnen VCG, Hoeben A, Vooijs MAGG. Non-invasive Glioblastoma Testing: Multimodal Approach to Monitoring and Predicting Treatment Response. Dis Markers. 2018 Jan 17;2018:2908609.

14. Eekers DBP, In 't Ven L, Deprez S, Jacobi L, Roelofs E, Hoeben A, Lambin P, de Ruysscher $D$, Troost EGC. The posterior cerebellum, a new organ at risk? Clin Transl Radiat Oncol. 2017 Nov 23;8:22-26.

15. Bouwens van der Vlis TAM, Hoeben A, Beckervordersandforth JC, Ackermans L, Eekers DBP, Wennekes RMJ, Schijns OEMG. Impact of the revised WHO classification of diffuse low-grade glioma on clinical decision making: A case report. Surg Neurol Int. 2017 Sep 7;8:223.

16. Zindler JD, Bruynzeel AME, Eekers DBP, Hurkmans CW, Swinnen A, Lambin P. Whole brain radiotherapy versus Stereotactic Radiosurgery for 4 - 10 brain metastases: a phase III randomized multicenter trial. BMC Cancer (2017) 17:500.

17. Klein M, Drijver J, Van den Bent M, Hoang-Xuan K, Taphoorn MJB, Reijneveld JC, Ben Hassel M, Eekers DBP et al. NCOG-7. Memory functioning in low grade glioma patienst treated with either radiotherapy (RT) of temozolomide (TZ) chemotherapy. A correlatiove analysis of European organization for research and treatment (EORTC) study 22033-26033. Neuro-Oncol. 2017;19:139. doi:.org/10.1093/neuonc/nox168.568.

18. Zindler JD, Jochems $A$, Beumer R, Lagerwaard $F$, Beumer $R$, Troost EGC, Eekers DBP, Compter I, Toorn van der P, Essers M, Oei B, Hurkmans C, Bruynzeel A, Bosmans G, Swinnen A, Leijneaar RTH, Lambin P. Individualized early death and long-term survival prediction after stereotactic radiosurgery for brain metastases of non-small cell lung cancer: Two externally validated nomograms. Radiother Oncol. 2017 May;123(2):189-194. 
19. Perry JR, Laperriere N, O'Callaghan CJ, Brandes AA, Menten J, Phillips C, Fay M, Nishikawa R, Cairncross JG, Roa W, Osoba D, Rossiter JP, Sahgal A, Hirte H, LaigleDonadey F, Franceschi E, Chinot O, Golfinopoulos V, Fariselli L, Wick A, Feuvret L, Back M, Tills M, Winch C, Baumert BG, Wick W, Ding K, Mason WP; Trial Investigators (Eekers DBP) Short-Course Radiation plus Temozolomide in Elderly Patients with Glioblastoma. N Engl J Med. 2017 Mar 16;376(11):1027-1037.

20. Verduin M, Zindler JD, Martinussen HMA, Jansen RLH, Croes S, Hendriks LEL, Eekers DBP, Hoeben A. Use of systemic therapy concurrent with cranial radiotherapy for cerebral metastases of solid tumors. Oncologist. 2017 Feb;22(2):222-235.

21. Lambin P, Zindler J, Vanneste B, Van De Voorde L, Eekers DBP, Compter I, Panth K Peerlings J, Larue RT, Deist TM, Jochems A, Lustberg T, van Soest J, de Jong EE, Even AJ, Reymen B, Rekers N, van Gisbergen M, Roelofs E, Carvalho S, Leijenaar RT, Zegers CM, Jacobs $M$, van Timmeren J, Brouwers $P$, Lal JA, Dubois L, Yaromina A, Van Limbergen EJ, Berbee $M$, van Elmpt W, Oberije C, Ramaekers B, Dekker A, Boersma LJ, Hoebers F, Smits KM, Berlanga AJ, Walsh S. Decision Support Systems for Personalized and Participative Radiation Oncology. Advanced Drug Delivery Reviews. Volume 109, 15 January 2017, Pages 131-153.

22. Eekers DBP, Roelofs E, Jelen U, Kirk M, Granzier M, Ammazzalorso F, Ahn PH, Janssens GO, Hoebers FJ, Friedmann T, Solberg T, Walsh S, Troost EG, Kaanders JH, Lambin P. Benefit of particle therapy in re-irradiation of head and neck patients. Results of a multicentric in silico ROCOCO trial. Radiotherapy \& Oncology. 121 Issue 3, December 2016, Pages 387-394.

23. Eekers DBP, Roelofs E, Jelen U Kirk, M, Granzier, M, Ammazzalorso, F, Ahn, PH, Janssens, GORJ, Hoebers, F, Friedmann, T, Solberg, T, Walsh, S, Troost, EGC, Kaanders, JHAM, Lambin, P. Data from: Benefit of particle therapy in re-irradiation of head and neck patients. Results of a multicenter in silico ROCOCO trial. 2016. doi:10.17195/candat.2016.04.2.

24. Zegers CML, Hoebers FCP, Elmpt van W, Bons JA, Öllers MC, Troost EGC, Eekers DBP, Balmaekers L, Arts-Pechtold M, Mottaghy FM, Lambin P. Evaluation of tumour hypoxia during radiotherapy using [18F]HX4 PET imaging and blood biomarkers in patients with head and neck cancer. EJNMMI. 2016 Nov;43(12):2139-2146.

25. Yahyanejad S, King H, Iglesias VS, Granton PV, Barbeau LMO, van Hoof SJ, Groot AJ, Habets R, Prickaerts J, Chalmers AJ, Eekers DBP, Theys J, Short SC, Verhaegen F, Vooijs $\mathrm{M}$. NOTCH blockade combined with radiation therapy and temozolomide prolongs survival of orthotopic glioblastoma. Oncotarget. 2016 Jul 5;7(27):4125141264. 
26. Compter I, Peerlings J, Eekers DBP, Postma AA, Ivanov D, Wiggins CJ, Kubben P, Küsters B, Wesseling P, Ackermans L, Schijns OE, Lambin P, Hoffmann AL. Technical feasibility of integrating $7 \mathrm{~T}$ anatomical MRI in image-guided radiotherapy of glioblastoma: a preparatory study. MAGMA Magnetic Resonance Materials in Physics, Biology and Medicine. MAGMA. 2016 Jun;29(3):591-603.

27. Cheng $Q$, Roelofs $E$, Ramaekers BLT, Eekers DBP, van Soest J, Lustberg $T$, Hendriks T, Hoebers F, van der Laan HP, Korevaar EW, Dekker A, Langendijk JA, Lambin P. Development and Evaluation of an Online Three-Level Proton vs Photon Decision Support Prototype for Head and Neck Cancer - Comparison of Dose, Toxicity and Cost-Effectiveness. Radiotherapy and Oncology 118 (2016) 281-285.

28. Lambin P, Zindler J, Vanneste B, van de Voorde L, Jacobs M, Eekers DBP, Peerlings J, Reymen B, Larue RT, Deist TM, de Jong EE, Even AJ, Berlanga AJ, Roelofs E, Cheng Q, Carvalho S, Leijenaar RT, Zegers CM, van Limbergen E, Berbee $M$, van Elmpt W, Oberije C, Houben R, Dekker A, Boersma L, Verhaegen F, Bosmans G, Hoebers F, Smits K, Walsh S. Modern clinical research: How rapid learning health care and cohort multiple randomised clinical trials complement traditional evidence based medicine. Acta Oncol. 2015 Oct;54(9):1289-300.

29. Hendriks LE, Schoenmaekers J, Zindler JD, Eekers DBP, Hoeben A, De Ruysscher DK, Dingemans AM. Safety of cranial radiotherapy concurrent with tyrosine kinase inhibitors in non-small cell lung cancer patients: A systematic review. Cancer Treat Rev. 2015 Jul;41(7):634-45.

30. Braeken AP, Kempen GI, Eekers DBP, Houben RM, van Gils FC, Ambergen T, Lechner L. Psychosocial screening effects on health-related outcomes in patients receiving radiotherapy. A cluster randomised controlled trial. Psychooncology. 2013 Dec;22(12):2736-46.

31. Braeken AP, Lechner L, Eekers DBP, Houben RM, van Gils FC, Ambergen T, Kempen GI. Does routine screening for psychosocial problems improve referral to caregivers and patient-radiotherapist communication? A cluster randomized controlled trial. Patient Educ Couns. 2013 Nov;93(2):289-97.

32. Braeken AP, Kempen GI, Eekers DBP, van Gils FC, Houben RM, Lechner L. The usefulness and feasibility of a screening instrument to identify psychosocial problems in patients receiving curative radiotherapy: a process evaluation. BMC Cancer 2011, 11:479.

33. Braeken AP, Lechner L, van Gils FC, Houben RM, Eekers DBP, Ambergen T, Kempen G. The effectiveness of the Screening Inventory Psychosocial Problems (SIPP) in cancer patients treated with radiotherapy: design of a cluster randomised controlled trial. BMC Cancer 2009,9:177. 
34. Eekers DBP, Laschet A, de Groot M, Roelofs E, Kester A, Delaere K, Lambin P, van Gils F, Nap M, ten Kate J. Why determine only the total prostate-specific antigen, if the free-to-total ratio contains the information? Ann Clin Biochem 2008;45:270274.

35. Kate ten J, Eekers DBP, de Groot M, Nap M, van Gils F. Bij welke totaal-PSAgrenzen heeft de F/T-ratio meerwaarde. Ned Tijdschr Klin Chem Labgeneesk 2005; 30: 18-19.

36. Schyns LEJR, van der Heyden B, Almeida IPO, Vaniqui A, Eekers DBP, Verhaegen F. Human tissue compositions and densities revisited after 60 years: an in vivo dual energy CT study for radiotherapy. Submitted 2018.

37. Jaspers J, Méndez Romero $A$, Hoogeman $M$, van den Bent $M$, Wiggenraad $M$, Taphoorn M, Lagerwaard F, Lucas A, Eekers DBP, Baumert B, Klein M. The hippocampal NTCP model could not be validated within the EORTC-22033 lowgrade glioma trial. Submitted 2018.

38. van der Heyden B, Wohlfahrt P, Troost EGC, Terhaag K, Eekers DBP, et al. Multiatlas and deep learning based organ-at-risk segmentation in neurological cancer patients using dual-energy CT images. Submitted 2018.

39. van der Heyden B, Almeida IP, Vilches-Freixas G, Van Beveren C, Vaniqui A, Ares MC, Terhaag K, Eekers DBP, Verhaegen F. Quantitative mass density determination: a comparison study between different single- and dual energy CT extraction methods for proton treatment planning in neurological radiotherapy. Submitted 2018. 
